\title{
HYPERPLANE SECTIONS AND DERIVED CATEGORIES
}

\author{
ALEXANDER KUZNETSOV
}

\begin{abstract}
We give a generalization of the theorem of Bondal and Orlov about the derived categories of coherent sheaves on intersections of quadrics revealing its relation to projective duality. As an application we describe the derived categories of coherent sheaves on Fano 3-folds of index 1 and degrees 12, 16 and 18.
\end{abstract}

\section{Contents}

Introduction 2

1. Statement of results $\quad 5$

2. Homological background $\quad 8$

2.1. Kernel functors $\quad 9$

2.2. Perfect spanning classes 12

2.3. Koszul complexes 13

2.4. Exact cartesian squares 13

2.5. Derived categories over a base 17

2.6. Faithful base changes and kernel functors $r$

2.7. Relative Serre functor and Bridgeland's trick 21

3. The universal hyperplane section $\quad 22$

3.1. Notation 22

3.2. Preparations 23

3.3. Proof of theorem $3.1 \quad 28$

4. Semiorthogonal decompositions 29

4.1. Universal families of linear sections 29

4.2. Preparations 31

4.3. Semiorthogonal collections for linear sections of $X$

4.4. Fullness 36

4.5. Exceptional collection for $Y \quad 38$

4.6. Semiorthogonal decompositions for linear section of $Y \quad 41$

4.7. Proof of the main theorem 43

5. General construction of $Y \quad 43$

$\begin{array}{lr}\text { 6. Examples } & 48\end{array}$

6.1. Grassmannian $\quad 48$

6.2. Orthogonal Grassmannian $\quad 48$

6.3. Lagrangian Grassmannian $\quad 49$

6.4. $G_{2}$ Grassmannian $\quad 50$

6.5. Intersection of quadrics $\quad 51$

Appendix A. Lagrangian Grassmannian $\quad 51$

$\begin{array}{ll}\text { Appendix B. } G_{2} \text { Grassmannian } & 58\end{array}$

Appendix C. Intersection of quadrics in $\mathbb{P}^{5} \quad 63$

Appendix D. Azumaya algebraic varieties 66

$\begin{array}{ll}\text { References } & 75\end{array}$

I was partially supported by RFFI grants 02-01-00468 and 02-01-01041, Russian Presidential grant for young scientists No. MK-3926.2004.1, CRDF Award No. RM1-2405-MO-02, and the Russian Science Support Foundation. 


\section{INTRODUCTION}

One of the most promising directions in modern algebraic geometry is the systematic use of derived categories of coherent sheaves [BO2]. Derived categories introduced by Verdier back in 1967 recently attracted a lot of attention due to the progress in understanding of their role in the modern geometry. It was realized that geometric similarity between different algebraic varieties sometimes is only the tip of the iceberg and is a consequence of a deeper relation which can be expressed as an equivalence of appropriate categories. Take for example the McKay correspondence. The story began in 1980 with John McKay's discovery [McK] of a correspondence between irreducible representations of a finite subgroup $\Gamma \subset \mathrm{SL}(2, \mathbb{C})$ and vertices of an affine Coxeter-Dynkin diagram of type $A, D, E$. A geometric interpretation of this correspondence incorporating a resolution of the simple surface singularity $\mathbb{C}^{2} / \Gamma$ was given by Gonzalez-Sprinberg and Verdier [GSV] in 1983. Kapranov and Vasserot [KV] have shown that the derived category of coherent sheaves on the resolution of $\mathbb{C}^{2} / \Gamma$ is equivalent to the derived category of coherent $\Gamma$-equivariant sheaves on $\mathbb{C}^{2}$. See also $[\mathrm{BKR}]$ for a beautiful generalization.

Another example of this sort is the theory of intersections of quadrics. Classically, it was formulated as a correspondence between intersections of quadrics and determinantal loci in the corresponding linear spaces of quadrics $[\mathrm{T}]$. The geometric meaning of this correspondence was discovered in [DR]. In the particular case of the intersection of a pencil of even-dimensional quadrics one should consider the hyperelliptic curve, obtained as a twofold covering of $\mathbb{P}^{1}$ parameterizing quadrics in the pencil with ramification at the points of $\mathbb{P}^{1}$ corresponding to degenerate quadrics. It was shown in loc. cit. that the moduli spaces of rank 2 vector bundles on this hyperelliptic curve can be described in terms of linear subspaces in the intersection of quadrics. Bondal and Orlov in [BO1] gave a categorical meaning to this by proving that the category of coherent sheaves on the hyperelliptic curve embeds fully and faithfully into the derived category of the intersection of quadrics. Finally, Bondal and Orlov [BO2, BO4] stated a theorem describing the structure of the derived category of a complete intersection of any number of quadrics. Roughly speaking it says that for any family of quadrics $L \subset S^{2} W^{*}$ in an even-dimensional vector space $W$ there exists a sheaf of finite algebras $\mathcal{A}_{L}$ on the twofold covering of $\mathbb{P}(L)$ ramified at the degeneration locus such that

Theorem 1 ([BO2, BO4]). If $2 \operatorname{dim} L<\operatorname{dim} W$ then there is a semiorthogonal decomposition

$$
\mathcal{D}^{b}\left(X_{L}\right)=\left\langle\mathcal{D}^{b}\left(\operatorname{Coh}\left(\mathcal{A}_{L}\right)\right), \mathcal{O}_{X_{L}}, \ldots, \mathcal{O}_{X_{L}}(\operatorname{dim} W-2 \operatorname{dim} L-1)\right\rangle,
$$

and if $2 \operatorname{dim} L=\operatorname{dim} W$ then there is an equivalence of categories $\mathcal{D}^{b}\left(X_{L}\right) \cong \mathcal{D}^{b}\left(\operatorname{Coh}\left(\mathcal{A}_{L}\right)\right)$, where $X_{L}$ stands for the complete intersection of quadrics in the family $L$, and $\mathcal{D}^{b}\left(\operatorname{Coh}\left(\mathcal{A}_{L}\right)\right)$ stands for the derived category of sheaves of coherent $\mathcal{A}_{L}$-modules.

Let us explain the relation of this theorem to projective duality.

Note that the intersection of quadrics $X_{L}$ can be considered as a linear section of the Veronese subvariety $X=\mathbb{P}(W) \subset \mathbb{P}\left(S^{2} W\right)$ by the linear space $\mathbb{P}\left(L^{\perp}\right) \subset \mathbb{P}\left(S^{2} W\right)$, where $L^{\perp} \subset S^{2} W^{*}$ is the orthogonal subspace to $L \subset S^{2} W^{*}$. On the other hand, the sheaf of algebras $\mathcal{A}_{L}$ on $\mathbb{P}(L) \subset \mathbb{P}\left(S^{2} W^{*}\right)$ can be extended to a sheaf $\mathcal{A}_{\mathbb{P}\left(S^{2} W^{*}\right)}$ on the whole $\mathbb{P}\left(S^{2} W^{*}\right)$. Actually, this sheaf is just the sheaf of even parts of the universal Clifford algebra, i.e.

$$
\mathcal{A}_{\mathbb{P}\left(S^{2} W^{*}\right)}=\mathcal{O}_{\mathbb{P}\left(S^{2} W^{*}\right)} \oplus \Lambda^{2} W \otimes \mathcal{O}_{\mathbb{P}\left(S^{2} W^{*}\right)}(-1) \oplus \cdots \oplus \Lambda^{2 n} W \otimes \mathcal{O}_{\mathbb{P}\left(S^{2} W^{*}\right)}(-n)
$$

with the Clifford multiplication. Consider $\mathbb{P}\left(S^{2} W^{*}\right)$ with the sheaf of algebras $\mathcal{A}_{\mathbb{P}\left(S^{2} W^{*}\right)}$ as a noncommutative algebraic variety, in fact a noncommutative finite covering of $\mathbb{P}\left(S^{2} W^{*}\right)$. One of consequences of theorem 1 which we would like to stress on is

the set of critical values of the projection $\left(\mathbb{P}\left(S^{2} W^{*}\right), \mathcal{A}_{\mathbb{P}\left(S^{2} W^{*}\right)}\right) \rightarrow \mathbb{P}\left(S^{2} W^{*}\right)$ coincides with the projectively dual variety $X^{\vee} \subset \mathbb{P}\left(S^{2} W^{*}\right)$. 
Indeed, since the projection $\left(\mathbb{P}\left(S^{2} W^{*}\right), \mathcal{A}_{\mathbb{P}\left(S^{2} W^{*}\right)}\right) \rightarrow \mathbb{P}\left(S^{2} W^{*}\right)$ is flat, a point $H \in \mathbb{P}\left(S^{2} W^{*}\right)$ is its critical value if and only if the fiber is singular. The singularity of an algebraic variety is equivalent to Extboundedness of its derived category of coherent sheaves. Thus the set of critical values coincides with the set of $H \in \mathbb{P}\left(S^{2} W^{*}\right)$ such that the category $\mathcal{D}^{b}\left(\operatorname{Coh}\left(\mathcal{A}_{H}\right)\right)$ is not Ext-bounded. Looking at the claim of theorem 1 we see that this happens if and only if the category $\mathcal{D}^{b}\left(X_{H}\right)$ is not Ext-bounded, i.e. if $X_{H}$ is singular. But $X_{H}$ is the hyperplane section of $X \subset \mathbb{P}\left(S^{2} W\right)$ by the hyperplane $H \in \mathbb{P}\left(S^{2} W^{*}\right)$, so recalling the definition of the projective duality we see that the set of critical values coincides with the projectively dual variety.

In fact, from the homological point of view the noncommutative variety $\left(\mathbb{P}\left(S^{2} W^{*}\right), \mathcal{A}_{\mathbb{P}\left(S^{2} W^{*}\right)}\right)$ is a better candidate to be called the projective dual of $X$ then $X^{\vee}$. It not only describes the set of singular hyperplane sections but also remembers nontrivial parts of their derived categories (for example, the triangulated category of singularities [O3] of $X_{H}$ is equivalent to the triangulated category of singularities of the fiber of the projection $\left(\mathbb{P}\left(S^{2} W^{*}\right), \mathcal{A}_{\mathbb{P}\left(S^{2} W^{*}\right)}\right) \rightarrow \mathbb{P}\left(S^{2} W^{*}\right)$ at $\left.H\right)$, in particular contains a lot of information about the singularities they have. So, it would be reasonable to say that $\left(\mathbb{P}\left(S^{2} W^{*}\right), \mathcal{A}_{\mathbb{P}\left(S^{2} W^{*}\right)}\right)$ is a Homologically Projectively Dual variety of $X$. The above considerations suggest that given an algebraic variety $X \subset \mathbb{P}(V)$, its homologically projectively dual variety should be defined as an algebraic variety $Y$ with a morphism $g: Y \rightarrow \mathbb{P}\left(V^{*}\right)$ and a sheaf of noncommutative algebras $\mathcal{A}_{Y}$ on $Y$, such that for any hyperplane $H \subset V^{*}$ the nontrivial part of the derived category $\mathcal{D}^{b}\left(X_{H}\right)$ is equivalent to the derived category $\mathcal{D}^{b}\left(Y_{H}, \mathcal{A}_{Y}\right)$ (roughly speaking, there exists a fully faithful embedding $\mathcal{D}^{b}\left(Y_{H}, \mathcal{A}_{Y}\right) \rightarrow \mathcal{D}^{b}\left(X_{H}\right)$ and the orthogonal to $\mathcal{D}^{b}\left(Y_{H}, \mathcal{A}_{Y}\right)$ in $\mathcal{D}^{b}\left(X_{H}\right)$ is Ext-bounded).

I am convinced that the Homological Projective Duality exists for a large class of algebraic varieties. In this paper we show how to construct a homologically projectively dual for a small class of varieties. Assume that $X$ is a smooth projective variety admitting an exceptional pair of vector bundles $\left(E_{1}, E_{2}\right)$ such that

$$
\left(E_{1} \otimes \mathcal{O}_{\mathbb{P}(V) \mid X}(1), E_{2} \otimes \mathcal{O}_{\mathbb{P}(V) \mid X}(1), \ldots, E_{1} \otimes \mathcal{O}_{\mathbb{P}(V) \mid X}(i), E_{1} \otimes \mathcal{O}_{\mathbb{P}(V) \mid X}(i)\right)
$$

is an exceptional collection, where $i$ is the index of $X$ (i.e. $\left.\omega_{X} \cong \mathcal{O}_{\mathbb{P}(V)}(-i)_{\mid X}\right)$. In this case $Y$ can be constructed as a moduli space of stable representations $\left(R_{1}, R_{2}\right)$ of the quiver

$$
\mathrm{Q}=\bullet \stackrel{\operatorname{Hom}\left(E_{1}, E_{2}\right)^{*}}{\longrightarrow} \bullet
$$

such that the cokernel of the corresponding map $R_{1} \otimes E_{1} \rightarrow R_{2} \otimes E_{2}$ is supported on a hyperplane section of $X$. The moduli space $Y$ carries a sheaf of Azumaya algebras $\mathcal{A}_{Y}$ and a universal family of representations $\left(F_{1}, F_{2}\right)$ in the category of $\mathcal{A}_{Y}$-modules (the Azumaya algebra $\mathcal{A}_{Y}$ corresponds to the element in the Brauer group which is the obstruction to the existence of a universal family, so in the category of $\mathcal{A}_{Y}$-modules the universal family exists). Moreover, associating to a point of $Y$ the support hyperplane of the cokernel of the corresponding map $R_{1} \otimes E_{1} \rightarrow R_{2} \otimes E_{2}$ we obtain a projection $g: Y \rightarrow \mathbb{P}\left(V^{*}\right)$. Denoting as above $X_{L}=X \cap \mathbb{P}\left(L^{\perp}\right), Y_{L}=Y \times_{\mathbb{P}\left(V^{*}\right)} \mathbb{P}(L)=g^{-1}(\mathbb{P}(L))$ we prove that:

Theorem 2. Assume that both $X_{L}$ and $Y_{L}$ are compact and have expected dimension. If $r=\operatorname{dim} L \leq i$ then there is a semiorthogonal decomposition

$$
\mathcal{D}^{b}\left(X_{L}\right)=\left\langle\mathcal{D}^{b}\left(Y_{L}, \operatorname{Coh}\left(\mathcal{A}_{Y}\right)\right), E_{1}(1), E_{2}(1), \ldots, E_{1}(i-r), E_{2}(i-r)\right\rangle,
$$

and if $r=\operatorname{dim} L \geq i$ then there is a semiorthogonal decomposition

$$
\mathcal{D}^{b}\left(Y_{L}, \mathcal{A}_{Y}\right)=\left\langle\mathcal{D}^{b}\left(X_{L}\right), F_{2}^{*}(i-r), F_{1}^{*}(i-r), \ldots, F_{2}^{*}(-1), F_{1}^{*}(-1)\right\rangle .
$$

In particular, for $\operatorname{dim} L=i$ we have an equivalence of categories $\mathcal{D}^{b}\left(X_{L}\right) \cong \mathcal{D}^{b}\left(Y_{L}, \operatorname{Coh}\left(\mathcal{A}_{Y}\right)\right)$. 
In fact instead of giving a direct proof we extract a list of conditions on $X,\left(E_{1}, E_{2}\right),\left(Y, \mathcal{A}_{Y}\right)$, and $\left(F_{1}, F_{2}\right)$ which imply the claim of the theorem. In some cases (when one can guess what $Y$ is) this allows to skip the construction of $Y$ at all. The proof of the theorem splits into two principal parts. In the first part we check the case $\operatorname{dim} L=1$, the case of hyperplane sections of $X$. In the other words, we prove there that $Y$ is homologically projectively dual to $X$. It turns out that the second part, the case of $\operatorname{dim} L>1$, follows from the first part more or less formally. Thus the above theorem in a sense is a consequence of homological projective duality.

A few words about the assumptions on the subspace $L$ made in the theorem. First of all, the compactness of $X_{L}$ is not a restriction, since $X$ is projective. On the other hand, the compactness of $Y_{L}$ is the most serious restriction. The problem is that $Y$ may not be compact (we constructed $Y$ as a moduli space of stable representations, and stability is an open condition). In fact, this problem arises because of imperfection of the construction of $Y$ (see below). In fact we expect that it is always possible to construct a compact $Y$ such that the theorem holds. In this case the assumption of compactness of $Y_{L}$ will hold automatically. Finally, the assumptions on the dimension of $X_{L}$ and $Y_{L}$ also can be taken off. However in this case we must be more accurate in the definition of $X_{L}$ and $Y_{L}$. In fact, the correct definition of both $X_{L}$ and $Y_{L}$ is the "derived" fiber product $X_{L}=X \times \times_{\mathbb{P}(V)}^{\mathbb{L}} \mathbb{P}\left(L^{\perp}\right), Y_{L}=Y \times \times_{\mathbb{P}\left(V^{*}\right)}^{\mathbb{L}} \mathbb{P}(L)$. As a topological space it coincides with the usual fiber product, but in a contrast with the usual case it is ringed with a certain sheaf of $D G$-algebras instead of the usual sheaf of functions. Correspondingly, the categories $\mathcal{D}^{b}\left(X_{L}\right)$ and $\mathcal{D}^{b}\left(Y_{L}, \mathcal{A}_{Y}\right)$ are appropriate categories of $D G$-modules. For instance, the above theorem allows to describe a certain category of $D G$-modules on any noncomplete intersection of quadrics. Since any projective variety in sufficiently ample embedding is an intersection of quadrics, this point of view seems to be very prominent.

Now let us discuss some formal consequences of the above theorem. First of all, we would like to mention that we haven't assumed the fullness of the exceptional collection on $X$ we have started from. However, take $L=0$, then $X_{L}=X, Y_{L}=\emptyset$ and it follows that the collection is full. Thus we have obtained a rather unexpected corollary. If a Fano variety admits an exceptional collection of the form $(*)$ then $(*)$ is full. Similarly, assume that $Y$ is compact and take $L=V^{*}$. Then $X_{L}=\emptyset, Y_{L}=Y$ and it follows that the collection

$$
\left(F_{2}^{*}(i-N), F_{1}^{*}(i-N), \ldots, F_{2}^{*}(-1), F_{1}^{*}(-1)\right),
$$

where $N=\operatorname{dim} V$ is a full exceptional collection in $\mathcal{D}^{b}\left(Y, \mathcal{A}_{Y}\right)$.

It also worth mentioning that the above theorem holds not only for individual linear sections of $X$ and $Y$, but also for families. In fact, the proof is based on investigation of families. For example, for the universal family $\mathcal{X}_{1}$ of hyperplane sections of $X$ (which is a divisor of bidegree $(1,1)$ in $X \times \mathbb{P}\left(V^{*}\right)$ ) the family $\mathcal{Y}_{1} \subset Y \times \mathbb{P}\left(V^{*}\right)$ of orthogonal linear sections of $Y$ coincides with the graph of the projection $g$. The relative version of the theorem in this cases claims that there is a semiorthogonal decomposition

$$
\mathcal{D}^{b}\left(\mathcal{X}_{1}\right)=\left\langle\mathcal{D}^{b}\left(Y, \mathcal{A}_{Y}\right), \mathcal{D}^{b}\left(\mathbb{P}\left(V^{*}\right)\right) \otimes E_{1}(1), \ldots, \mathcal{D}^{b}\left(\mathbb{P}\left(V^{*}\right)\right) \otimes E_{2}(i-1)\right\rangle
$$

if $Y$ is compact. This decomposition suggests a categorical definition of the compactification $\bar{Y}$ of $Y$ :

$$
\mathcal{D}^{b}\left(\bar{Y}, \mathcal{A}_{\bar{Y}}\right)=\left\langle\mathcal{D}^{b}\left(\mathbb{P}\left(V^{*}\right)\right) \otimes E_{1}(1), \ldots, \mathcal{D}^{b}\left(\mathbb{P}\left(V^{*}\right)\right) \otimes E_{2}(i-1)\right\rangle^{\perp} \subset \mathcal{D}^{b}\left(\mathcal{X}_{1}\right) .
$$

In fact, one could take this for the definition of the homologically projectively dual variety of $X$, if only there were a possibility of proving that the above category is geometric.

Now let us describe what our approach gives in the case we have started from, i.e. for the intersection of even-dimensional quadrics. In this case we take $X=\mathbb{P}(W), W$ is even-dimensional, the Veronese embedding $X \subset \mathbb{P}\left(S^{2} W\right)=\mathbb{P}(V)$, and the exceptional pair $\left(E_{1}, E_{2}\right)=\left(\mathcal{O}_{\mathbb{P}(W)}, \mathcal{O}_{\mathbb{P}(W)}(1)\right)$ (note that $\mathcal{O}_{\mathbb{P}(V) \mid X}(1) \cong \mathcal{O}_{\mathbb{P}(W)}(2)$, so the collection $(*)$ is the standard exceptional collection on $\left.\mathbb{P}(W)\right)$. The 
discussed above moduli space of representations of the quiver however doesn't give the whole $\mathbb{P}\left(S^{2} W^{*}\right)$, but only the open part $Y=\mathbb{P}\left(S^{2} W^{*}\right) \backslash \mathbf{Z}$, where $\mathbf{Z}$ is the locus of quadrics of corank 2. Thus, rigourously speaking at the moment our method proves theorem 1 only for $\operatorname{dim} L \leq 3$.

One of explanations why the quiver approach doesn't give the whole $\mathbb{P}\left(S^{2} W^{*}\right)$ at the output is the fact that the sheaf of algebras $\mathcal{A}_{\mathbb{P}\left(S^{2} W^{*}\right)}$ fails to be Azumaya over $\mathbf{Z}$, while using the quiver approach we only can get an Azumaya algebra. However, we expect that there exists a certain algebraic stack $\bar{Y}$ with the underlying algebraic variety $\mathbb{P}\left(S^{2} W^{*}\right)$ such that the sheaf of algebras $\mathcal{A}_{\mathbb{P}\left(S^{2} W^{*}\right)}$ comes from a sheaf of Azumaya algebras on $\bar{Y}$. This suggests that it might be better in general situation to consider a moduli stack of the quiver representations instead of the moduli space.

Correspondingly, in the general situation one can try to find an appropriate stack compactification $\bar{Y}$ of the moduli space $Y$ such that the sheaf of algebras $\mathcal{A}_{Y}$ (and the universal family as well) can be extended to a sheaf of Azumaya algebras $\mathcal{A}_{\bar{Y}}$.

Another possible approach to the construction of a Homologically Projectively Dual variety is to avoid the use of quivers at all and to construct $Y$ as a moduli spaces of appropriate objects (e.g. spinor bundles) in the derived category of coherent sheaves on $X$ which are supported on hyperplane sections of $X$.

In fact, in both cases the main obstacle is absence of a good categorical theory of moduli spaces (an output of a good theory of moduli spaces must be in general a noncommutative algebraic variety).

Now it is time to describe the contents of the paper. In section 1 we introduce the notation and state the main result of the paper, theorem 1.2. In section 2 we introduce the homological background. We define exact cartesian squares and faithful base changes in subsection 2.4 and prove the main technical results: the faithful base change theorem 2.45 and the relative version of the Bridgeland's trick 2.50. In section 3 we make the first step of the proof of theorem 1.2 and in section 4 we finish the proof. In section 5 we describe the general construction of $Y$ from $X$ via the quiver moduli spaces. In section 6 we give some examples where theorem 1.2 works. In appendices $\mathrm{A}, \mathrm{B}$ and $\mathrm{C}$ we work out some details relating to these examples. Finally, in appendix D we check the basic facts about the derived categories of coherent $\mathcal{A}$-modules, where $\mathcal{A}$ is a sheaf of Azumaya algebras. We pay also special attention to the relation of complexes of finite Tor and Ext-amplitude to perfect complexes.

Acknowledgements. I am grateful to A.Bondal, D.Orlov, D.Kaledin and A.Samokhin for useful discussions. Some of the results of this work have been presented at the conference "Noncommutative Algebra and Algebraic Geometry" in the University of Warwick. I would like to thank organizers for this possibility.

\section{Statement of Results}

As it was indicated in the introduction, we start with presenting a set of conditions on a pair of algebraic varieties $X$ and $Y$ implying their homological projective duality.

Assume that we have the following data:

(D.1) a smooth, projective variety $X$ with an algebraic morphism to a projective space $f: X \rightarrow \mathbb{P}(V)$;

(D.2) a pair of vector bundles $\left(E_{1}, E_{2}\right)$ on $X$;

(D.3) an algebraic variety $Y$ with a sheaf of Azumaya algebras $\mathcal{A}_{Y}$ and a projective morphism $g: Y \rightarrow \mathbf{P}=\mathbb{P}\left(V^{*}\right)-\mathbf{Z}$, where $\mathbf{Z} \subset \mathbb{P}\left(V^{*}\right)$ is a closed subset in the dual projective space;

(D.4) a pair of locally projective $\mathcal{A}_{Y}$-modules $\left(F_{1}, F_{2}\right)$ on $Y$;

(D.5) a linear morphism $\phi: \operatorname{Hom}\left(E_{1}, E_{2}\right)^{*} \rightarrow \operatorname{Hom}_{\mathcal{A}_{Y}}\left(F_{1}, F_{2}\right)$.

Given such a data we denote

- $W=\operatorname{Hom}\left(E_{1}, E_{2}\right)^{*}$;

- $N=\operatorname{dim} V$; 
- $Q \subset \mathbb{P}(V) \times \mathbb{P}\left(V^{*}\right)$ - the incidence quadric;

- $Q(X, Y)=(X \times Y) \times \mathbb{P}(V) \times \mathbb{P}\left(V^{*}\right) Q$ and $i: Q(X, Y) \rightarrow X \times Y$ - the embedding.

The incidence quadric $Q \subset \mathbb{P}(V) \times \mathbb{P}\left(V^{*}\right)$ is a divisor of bidegree $(1,1)$. Its fiber over a point $H \in \mathbb{P}\left(V^{*}\right)$ is the corresponding hyperplane in $\mathbb{P}(V)$ which we denote by the same letter $H \subset \mathbb{P}(V)$. Similarly, the fiber of $Q(X, Y)$ over a point $H \in \mathbb{P}\left(V^{*}\right)$ is the hyperplane section $X_{H}=X \cap H$ of $X$. Abusing the notation we call $Q(X, Y)$ the incidence quadric too. Note, that it follows from the condition (C.1) below that the incidence quadric $Q(X, Y)$ is a divisor of bidegree $(1,1)$ on $X \times Y$.

The map $\phi: W \rightarrow \operatorname{Hom}_{\mathcal{A}_{Y}}\left(F_{1}, F_{2}\right)=\operatorname{Hom}_{\mathcal{A}_{Y}}\left(F_{2}^{*}, F_{1}^{*}\right)$ induces the following evaluation homomorphisms $W \otimes F_{1} \rightarrow F_{2}$ and $W \otimes F_{2}^{*} \rightarrow F_{1}^{*}$. Tensoring the first by $F_{2}^{*}$ and the second by $F_{1}$ and summing up we obtain a homomorphism

- $W \otimes F_{2}^{*} \otimes F_{1} \stackrel{\operatorname{ad}(\phi)}{\longrightarrow} F_{2}^{*} \otimes F_{2} \oplus F_{1}^{*} \otimes F_{1}$ on $Y \times Y$.

On the other hand, composing the evaluation homomorphism $W \otimes F_{1} \rightarrow F_{2}$ tensored by $E_{2}$ with the coevaluation homomorphism $E_{1} \rightarrow W \otimes E_{2}$ tensored by $F_{1}$ we obtain a homomorphism

- $E_{1} \otimes F_{1} \longrightarrow E_{2} \otimes F_{2}$ on $X \times Y$.

Assume that our data satisfies the following list of conditions:

(C.1) $f(X)$ is not contained in a hyperplane in $\mathbb{P}(V)$;

(C.2) $\omega_{X} \cong \mathcal{O}_{X}(-i):=f^{*} \mathcal{O}_{\mathbb{P}(V)}(-i), i>0$

(C.3) $\left(E_{1}(1), E_{2}(1), \ldots, E_{1}(i), E_{2}(i)\right)$ is an exceptional collection,

(i.e. $\operatorname{Ext}^{\bullet}\left(E_{s}, E_{s}\right)=\mathrm{k}$ for $s=1,2$ and $\operatorname{Ext}^{\bullet}\left(E_{s}(k), E_{t}(l)\right)=0$ for $i \geq k>l \geq 1$ or $k=l$ and $s>t$ ), and additionally $\operatorname{Ext}^{>0}\left(E_{1}, E_{2}\right)=0$;

(C.4) $g$ is projective;

(C.5) Coker $\operatorname{ad}(\phi) \cong \Delta_{*} \mathcal{A}_{Y}$ on $Y \times Y$, where $\Delta: Y \rightarrow Y \times Y$ is the diagonal embedding;

(C.6) $\operatorname{Ker} e=0$ and Coker $e=i_{*} \mathcal{E}$, where $\mathcal{E}$ is a coherent $\mathcal{A}_{Y^{-}}$-module on $Q(X, Y)$;

(C.7) $\operatorname{dim} X+N-\operatorname{codim}_{Y \times Y}\left(Y \times_{\mathbb{P}\left(V^{*}\right)} Y\right)=2 i$;

(C.8) for any hyperplane $H \subset \mathbb{P}\left(V^{*}\right)$ we have $\operatorname{dim}(\mathbf{Z} \cap H) \leq N-i-2$.

The integer $i$ defined in condition (C.2) is called the index of $X$. If $\mathbf{Z}$ is irreducible and is not contained in a hyperplane in $\mathbb{P}\left(V^{*}\right)$ then the condition (C.8) is equivalent to $\operatorname{dim} \mathbf{Z} \leq N-i-1$.

For every linear subspace $L \subset V^{*}$ we denote $X_{L}=X \times_{\mathbb{P}(V)} \mathbb{P}\left(L^{\perp}\right)$ and $Y_{L}=Y \times_{\mathbb{P}\left(V^{*}\right)} \mathbb{P}(L)$.

Definition 1.1. A subspace $L \subset V^{*}$ is admissible, if

(a) $\operatorname{dim} X_{L}=\operatorname{dim} X-\operatorname{dim} L$,

(b) $\operatorname{dim} Y_{L}=\operatorname{dim} Y+\operatorname{dim} L-N$, and

(c) $\mathbb{P}(L) \cap \mathbf{Z}=\emptyset$.

Note that condition (c) above together with (C.4) implies that $Y_{L}$ is compact.

Theorem 1.2. If conditions (C.1)-(C.8) are satisfied then

1) $Y$ is smooth and $\omega_{Y} \cong \mathcal{O}_{Y}(i-N)$;

2) for any admissible subspace $L \subset V^{*}$ there exist semiorthogonal decompositions

$$
\begin{array}{lll}
\mathcal{D}^{b}\left(X_{L}\right) & =\left\langle\mathcal{D}^{b}\left(Y_{L}, \mathcal{A}_{Y}\right), E_{1}(1), E_{2}(1), \ldots, E_{1}(i-r), E_{2}(i-r)\right\rangle, & \text { if } r \leq i \\
\mathcal{D}^{b}\left(Y_{L}, \mathcal{A}_{Y}\right) & =\left\langle\mathcal{D}^{b}\left(X_{L}\right), F_{2}^{*}(i-r), F_{1}^{*}(i-r), \ldots, F_{2}^{*}(-1), F_{1}^{*}(-1)\right\rangle, & \text { if } r \geq i,
\end{array}
$$

where $r=\operatorname{dim} L$.

Now let us sketch the proof of the theorem, assuming for simplicity that $\mathbf{Z}=\emptyset$. First of all, to avoid problems which arise when one works with derived categories of singular varieties, we replace individual linear sections $X_{L}, Y_{L}$ of $X$ and $Y$ by the universal families of linear sections $\mathcal{X}_{r} \subset X \times \operatorname{Gr}\left(r, V^{*}\right)$, 
$\mathcal{Y}_{r} \subset Y \times \operatorname{Gr}\left(r, V^{*}\right)$, which enjoy the property to be totally smooth. We note that the natural projection $\mathcal{X}_{r} \times{ }_{\mathrm{Gr}\left(r, V^{*}\right)} \mathcal{Y}_{r} \rightarrow X \times Y$ factors through $Q(X, Y)$ and denote by $\mathcal{E}_{r}$ the pullback of the sheaf $\mathcal{E}$, defined by condition (C.6). Then the objects $\mathcal{E}_{r}$ on $\mathcal{X}_{r} \times \operatorname{Gr}\left(r, V^{*}\right) \mathcal{Y}_{r} \subset \mathcal{X}_{r} \times \mathcal{Y}_{r}$, considered as kernels, provide us with functors $\Phi_{r}: \mathcal{D}^{b}\left(\mathcal{Y}_{r}, \mathcal{A}_{Y}\right) \rightarrow \mathcal{D}^{b}\left(\mathcal{X}_{r}\right)$. Consider also their left and right adjoint functors $\Phi_{r}^{*}, \Phi_{r}^{!}$: $\mathcal{D}^{b}\left(\mathcal{X}_{r}\right) \rightarrow \mathcal{D}^{b}\left(\mathcal{Y}_{r}, \mathcal{A}_{Y}\right)$. Now the proof goes in several steps:

Step 1: We note that $\mathcal{Y}_{1}=Y$ and prove that the functor $\Phi_{1}: \mathcal{D}^{b}\left(Y, \mathcal{A}_{Y}\right) \rightarrow \mathcal{D}^{b}\left(\mathcal{X}_{1}\right)$ is fully faithful. This is done by explicit computation of $\Phi_{1}^{*} \circ \Phi_{1}$. The convolution of kernels of these functors is shown to be isomorphic to the sheaf Coker ad $(\phi)$ on $Y \times Y$, which by condition (C.4) gives the identity functor on $\mathcal{D}^{b}\left(Y, \mathcal{A}_{Y}\right)$, see details in section 3 .

Step 2: We prove that the functor $\Phi_{r}: \mathcal{D}^{b}\left(\mathcal{Y}_{r}, \mathcal{A}_{Y}\right) \rightarrow \mathcal{D}^{b}\left(\mathcal{X}_{r}\right)$ is fully faithful for all $r \leq i$ by induction in $r$ and that the collection

$$
\left\langle\mathcal{D}^{b}\left(\mathcal{Y}_{r}, \mathcal{A}_{Y}\right), \mathcal{D}^{b}\left(\operatorname{Gr}\left(r, V^{*}\right)\right) \otimes E_{1}(1), \ldots, \mathcal{D}^{b}\left(\operatorname{Gr}\left(r, V^{*}\right)\right) \otimes E_{2}(i-r)\right\rangle
$$

is semiorthogonal in $\mathcal{D}^{b}\left(\mathcal{X}_{r}\right)$, see details in subsection 4.3 .

Step 3: We use the Bridgeland's trick in relative situation. Note that the relative canonical bundle of $\mathcal{X}_{i}$ over $\operatorname{Gr}\left(i, V^{*}\right)$ is given by $\omega_{\mathcal{X}_{i} / \operatorname{Gr}\left(i, V^{*}\right)} \cong \operatorname{det} \mathcal{L}_{i}^{*}$, where $\mathcal{L}_{i}$ is the tautological rank $i$ subbundle on $\operatorname{Gr}\left(i, V^{*}\right)$. Thus $\mathcal{X}_{i}$ is a family of Calabi-Yau varieties over $\operatorname{Gr}\left(i, V^{*}\right)$. On the other hand, the functor $\Phi_{i}$ is $\operatorname{Gr}\left(i, V^{*}\right)$-linear (i.e. commutes with tensoring by pullbacks of bundles on $\operatorname{Gr}\left(i, V^{*}\right)$ ). We show in subsection 2.7 that a relative version of the Bridgeland's trick (proposition 2.50) works in this case, so $\Phi_{i}$ is an equivalence, and $\omega_{\mathcal{Y}_{i} / \operatorname{Gr}\left(i, V^{*}\right)} \cong \operatorname{det} \mathcal{L}_{i}^{*}$, see details in subsection 4.4 .

Step 4: We use descending induction in $r$ to check that $\omega_{\mathcal{Y}_{r} / \operatorname{Gr}\left(r, V^{*}\right)} \cong \operatorname{det} \mathcal{L}_{r}^{*}(i-r)$, where $\mathcal{L}_{r}$ is the tautological rank $r$ subbundle on $\operatorname{Gr}\left(r, V^{*}\right)$, see details in subsection 4.4.

Step 5: We use descending induction in $r$ to check that the above semiorthogonal collection in $\mathcal{D}^{b}\left(\mathcal{X}_{r}\right)$ is full. In other words, we prove that we have a semiorthogonal decomposition

$$
\mathcal{D}^{b}\left(\mathcal{X}_{r}\right)=\left\langle\mathcal{D}^{b}\left(\mathcal{Y}_{r}, \mathcal{A}_{Y}\right), \mathcal{D}^{b}\left(\operatorname{Gr}\left(r, V^{*}\right)\right) \otimes E_{1}(1), \ldots, \mathcal{D}^{b}\left(\operatorname{Gr}\left(r, V^{*}\right)\right) \otimes E_{2}(i-r)\right\rangle,
$$

for all $r \leq i$, see details in subsection 4.4.

Step 6: By induction in $r$ we show that for all $r \leq i$ the functors $\Phi_{r}^{!}: \mathcal{D}^{b}\left(\mathcal{X}_{r}\right) \rightarrow \mathcal{D}^{b}\left(\mathcal{Y}_{r}\right)$ take the exceptional pair $\left(E_{0}(1), E_{1}(1)\right)$ to the pair $\left(F_{2}^{*}, F_{1}^{*}\right)$ up to a twist and a shift, where $E_{0}$ is the mutation of $E_{2}$ through $E_{1}$, (that is $E_{0}$ is defined from the exact triangle $E_{0} \rightarrow W^{*} \otimes E_{1} \stackrel{\text { ev }}{\longrightarrow} E_{2}$ ). It follows that $\left\langle\mathcal{D}^{b}\left(\operatorname{Gr}\left(r, V^{*}\right)\right) \otimes F_{2}^{*}(i-r), \ldots, \mathcal{D}^{b}\left(\operatorname{Gr}\left(r, V^{*}\right)\right) \otimes F_{1}^{*}(-1)\right\rangle$ is a semiorthogonal collection in $\mathcal{D}^{b}\left(\mathcal{Y}_{r}, \mathcal{A}_{Y}\right)$ for all $r \geq i$. In particular, it follows that $\left(F_{2}^{*}(i-N), F_{1}^{*}(i-N), \ldots, F_{2}^{*}(-1), F_{1}^{*}(-1)\right)$ is an exceptional collection in $\mathcal{D}^{b}\left(Y, \mathcal{A}_{Y}\right)$ (if $\mathbf{Z}=\emptyset$ ), see details in subsection 4.5.

Step 7: We use ascending induction in $r$ to check that

$$
\mathcal{D}^{b}\left(\mathcal{Y}_{r}, \mathcal{A}_{Y}\right)=\left\langle\mathcal{D}^{b}\left(\mathcal{X}_{r}\right), \mathcal{D}^{b}\left(\operatorname{Gr}\left(r, V^{*}\right)\right) \otimes F_{2}^{*}(i-r), \ldots, \mathcal{D}^{b}\left(\operatorname{Gr}\left(r, V^{*}\right)\right) \otimes F_{1}^{*}(-1)\right\rangle
$$

for all $r \geq i$, see details in subsection 4.6.

The inductions steps are based on the following construction. Consider the partial flag variety $\mathrm{FI}(r-$ $\left.1, r ; V^{*}\right)$ and the tautological subbundles $\mathcal{L}_{r-1} \subset \mathcal{L}_{r} \subset V^{*} \otimes \mathcal{O}_{\mathrm{Fl}\left(r-1, r ; V^{*}\right)}$ of rank $(r-1)$ and $r$ on it. Let

$$
\begin{array}{ll}
\mathcal{X}_{\mathcal{L}_{r-1}}=\mathcal{X}_{r-1} \times{ }_{\operatorname{Gr}\left(r-1, V^{*}\right)} \mathrm{FI}\left(r-1, r ; V^{*}\right), & \mathcal{X}_{\mathcal{L}_{r}}=\mathcal{X}_{r} \times \operatorname{Gr}\left(r, V^{*}\right) \\
\mathcal{Y}_{\mathcal{L}_{r-1}}=\mathcal{Y}_{r-1} \times{ }_{\operatorname{Gr}\left(r-1, V^{*}\right)} \operatorname{FI}\left(r-1, r ; V^{*}\right), & \mathcal{Y}_{\mathcal{L}_{r}}=\mathcal{Y}_{r} \times{ }_{\operatorname{Gr}\left(r, V^{*}\right)} \operatorname{FI}\left(r-1, r ; V^{*}\right),
\end{array}
$$

Then $\mathcal{X}_{\mathcal{L}_{r}}$ is a divisor in $\mathcal{X}_{\mathcal{L}_{r-1}}, \mathcal{Y}_{\mathcal{L}_{r-1}}$ is a divisor in $\mathcal{Y}_{\mathcal{L}_{r}}$, and we have the following diagrams

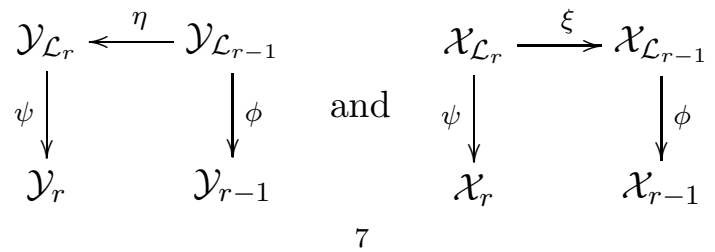


where morphisms $\xi$ and $\eta$ are divisorial embeddings, while $\phi$ and $\psi$ are the projections. Moreover, the pullbacks $\mathcal{E}_{\mathcal{L}_{r-1}}$ and $\mathcal{E}_{\mathcal{L}_{r}}$ of $\mathcal{E}_{r-1}$ and $\mathcal{E}_{r}$ via the projections $\mathcal{X}_{\mathcal{L}_{r-1}} \times{ }_{\mathrm{Fl}\left(r-1, r ; V^{*}\right)} \mathcal{Y}_{\mathcal{L}_{r-1}} \rightarrow \mathcal{X}_{r-1} \times{ }_{\mathrm{Gr}\left(r-1, V^{*}\right)}$ $\mathcal{Y}_{r-1}$ and $\mathcal{X}_{\mathcal{L}_{r}} \times_{\mathrm{FI}\left(r-1, r ; V^{*}\right)} \mathcal{Y}_{\mathcal{L}_{r}} \rightarrow \mathcal{X}_{r} \times{ }_{\mathrm{Gr}\left(r, V^{*}\right)} \mathcal{Y}_{r}$ provide us with the functors $\Phi_{\mathcal{L}_{r-1}}$ and $\Phi_{\mathcal{L}_{r}}$ between the corresponding derived categories. Since all these functors originate from the same object $\mathcal{E}$ on $Q(X, Y)$, we can find a certain relations between them, see details in subsection 4.2.

Finally, when steps 1-7 are performed, we can deduce the theorem. For the first claim we consider $r=1$ and note that $\mathcal{Y}_{1}=Y$. Hence we have a semiorthogonal decomposition

$$
\mathcal{D}^{b}\left(\mathcal{X}_{1}\right)=\left\langle\mathcal{D}^{b}\left(Y, \mathcal{A}_{Y}\right), \mathcal{D}^{b}\left(\mathbb{P}\left(V^{*}\right)\right) \otimes E_{1}(1), \ldots, \mathcal{D}^{b}\left(\mathbb{P}\left(V^{*}\right)\right) \otimes E_{2}(i-1)\right\rangle,
$$

and an isomorphism $\omega_{Y / \mathbb{P}\left(V^{*}\right)} \cong \mathcal{L}_{1}^{*}(i-1) \cong \mathcal{O}_{Y}(i)$. This immediately implies

$$
\omega_{Y} \cong \omega_{Y / \mathbb{P}\left(V^{*}\right)} \otimes \omega_{\mathbb{P}\left(V^{*}\right)} \cong \mathcal{O}_{Y}(i-N) .
$$

On the other hand, since $\mathcal{X}_{1}$ is smooth we deduce that $\mathcal{D}^{b}\left(Y, \mathcal{A}_{Y}\right)$ being a semiorthogonal component of an Ext-bounded category $\mathcal{D}^{b}\left(\mathcal{X}_{1}\right)$ is Ext-bounded, hence $\left(Y, \mathcal{A}_{Y}\right)$ is smooth.

Finally, the second claim of the theorem is deduced by a base change argument for the base change Speck $\rightarrow \operatorname{Gr}\left(r, V^{*}\right)$ corresponding to an admissible $r$-dimensional subspace $L \subset V^{*}$, using the faithful base change theorem 2.45 .

The next question we address is how to construct $Y$ starting from $X$. More precisely, assume that we have data (D.1) and (D.2) satisfying conditions (C.1)-(C.3). How to construct data (D.3)-(D.5) such that conditions (C.4)-(C.8) are satisfied? We propose the following approach. Since we must have a morphism $\phi_{1}: W \otimes F_{1} \rightarrow F_{2}$ on $Y$, it is natural to construct $Y$ as a subscheme in the moduli space of representations of the quiver $\mathrm{Q}=\bullet \stackrel{W}{\longrightarrow} \bullet$. The condition (C.6) suggests that the dimension vector $\left(d_{1}, d_{2}\right)$ of the representations must satisfy

$$
\theta\left(d_{1}, d_{2}\right)=d_{2} \operatorname{rank}\left(E_{2}\right)-d_{1} \operatorname{rank}\left(E_{1}\right)=0 .
$$

Note that by this condition the dimension vector is defined only up to a multiplicative constant. Let $\mathbf{R}_{m}$ denote the representation space of the quiver $\mathbf{Q}$ for the dimension vector $\left(d_{1}, d_{2}\right)$ satisfying the above condition with $\operatorname{gcd}\left(d_{1}, d_{2}\right)=m$, and let $\left(\mathcal{R}_{1}, \mathcal{R}_{2}\right)$ be the tautological family of representations. Then (C.6) suggests to consider the following GIT-quotient

$$
\bar{Y}_{m}=\left\{(\rho, H) \in \mathbf{R}_{m} \times \mathbb{P}\left(V^{*}\right) \mid \operatorname{supp} \operatorname{Coker}\left(E_{1} \otimes \mathcal{R}_{1 \rho} \stackrel{e_{\rho}}{\longrightarrow} E_{2} \otimes \mathcal{R}_{2 \rho}\right)=X \cap H\right\} / / \chi G,
$$

where $G=\mathrm{GL}\left(d_{1}\right) \times \mathrm{GL}\left(d_{2}\right) / \mathrm{k}^{*}$ and $\chi\left(g_{1}, g_{2}\right)=\operatorname{det}\left(g_{1}\right)^{-\operatorname{rank}\left(E_{1}\right)} \operatorname{det}\left(g_{2}\right)^{\operatorname{rank}\left(E_{2}\right)}$ is a character of $G$. Note that to obtain a universal family of quiver representations on $\bar{Y}_{m}$ we have to consider a sheaf of Azumaya algebras on $\bar{Y}_{m}$. Moreover, if we want (C.5) to be satisfied we have to restrict to the stable locus, thus replacing $\bar{Y}_{m}$ with $Y_{m}=g_{m}^{-1}\left(\mathbb{P}\left(V^{*}\right) \backslash \bar{Z}_{m}\right)$, where $g_{m}: \bar{Y}_{m} \rightarrow \mathbb{P}\left(V^{*}\right)$ is the canonical projection and $\bar{Z}_{m}=\cup_{2 k \leq m} g_{k}\left(\bar{Y}_{k}\right)$. Then under some mild additional assumptions we can show that (C.4)-(C.6) are indeed satisfied for $Y_{m}$. It remains to choose $m$ in such a way that (C.7) holds and to hope that (C.8) would be true for this $m$. This choice and verification of (C.7) are the most cumbersome part of the work and must be done for each case separately.

\section{HomologicAl BACKGROUnd}

This section is designed to develop some machinery for working with derived categories in relative situation. The main results are the faithful base change theorem 2.45 and a relative version of the Bridgeland's trick 2.50. Since we need these results to be applicable to the derived categories of sheaves of modules over sheaves of Azumaya algebras, we must work in the corresponding category.

We define an Azumaya variety $\left(X, \mathcal{A}_{X}\right)$ as an embeddable algebraic variety of finite type $X$ over a field equipped with a sheaf $\mathcal{A}_{X}$ of semisimple $\mathcal{O}_{X}$-algebras, such that $\mathcal{A}_{X}$ is locally free over $\mathcal{O}_{X}$. A morphism 
of Azumaya varieties $f:\left(X, \mathcal{A}_{X}\right) \rightarrow\left(Y, \mathcal{A}_{Y}\right)$ consists of a morphism $f_{\circ}: X \rightarrow Y$ of the underlying algebraic varieties and a homomorphism of $\mathcal{O}_{X}$-algebras $f_{\mathcal{A}}: f_{\circ}^{*} \mathcal{A}_{Y} \rightarrow \mathcal{A}_{X}$. Thus we treat Azumaya varieties as ringed spaces with $\mathcal{A}_{X}$ playing the role of a sheaf of rings. Azumaya varieties form a category, which contains the category of usual embeddable algebraic varieties as a full subcategory (the embedding functor takes $X$ to $\left.\left(X, \mathcal{O}_{X}\right)\right)$.

Below we will work in the category of Azumaya varieties. To unburden the notation we will often write $X$ instead of $\left(X, \mathcal{A}_{X}\right)$ when it is clear which sheaf of algebras is used, or when it doesn't matter. The necessary background on Azumaya varieties is contained in appendix D. We define there the functors $f_{*}$, $f^{*}, f^{!}, \otimes_{\mathcal{A}_{X}}$ and $\mathrm{RH} \mathrm{Hom}_{\mathcal{A}_{X}}$, and check that all usual relations between them are satisfied. Here we shall only mention that a morphism $f$ is called strict if $f_{\mathcal{A}}$ is an isomorphism, and that for a strict morphism $f$ we have $f^{*}=f_{\circ}^{*}$ and $f^{!}=f_{\circ}^{!}$.

The base field $\mathrm{k}$ is assumed to be an algebraically closed field of zero characteristic.

2.1. Kernel functors. We denote by $\mathcal{D}_{q c}^{b}(X), \mathcal{D}_{q c}^{-}(X), \mathcal{D}_{q c}^{+}(X)$ and $\mathcal{D}_{q c}(X)$ the bounded, the bounded above, the bounded below and the unbounded derived categories of quasicoherent sheaves on an Azumaya variety $X$. Further, $\mathcal{D}(X)$ stands for the unbounded derived category of quasicoherent sheaves on $X$ with coherent cohomologies, and similarly for $\mathcal{D}^{b}(X), \mathcal{D}^{-}(X)$ and $\mathcal{D}^{+}(X)$. Finally, $\mathcal{D}^{\text {perf }}(X)$ stands for the category of perfect complexes.

Whenever we wish to emphasize the role of an Azumaya algebra on $X$, or want to point out it explicitly, we use the notation $\mathcal{D}\left(X, \mathcal{A}_{X}\right)$ e.t.c.

For an object $F \in \mathcal{D}(X)$ we denote by $\mathcal{H}^{i}(F)$ the $i$-th cohomology sheaf of $F$. For any integers $a \leq b$ we denote by $\mathcal{D}^{[a, b]}(X)$ the full subcategory of $\mathcal{D}(X)$ formed by objects $F$ such that $\mathcal{H}^{i}(F)=0$ for $i \notin[a, b]$. Similarly, we define $\mathcal{D}^{\geq a}(X)$ and $\mathcal{D}^{\leq a}(X)$. For a morphism $f: X \rightarrow Y$ we denote by $f_{*}, f^{*}$ and $f^{!}$the derived pushforward, the derived pullback and the twisted pullback functors. Similarly, $\otimes_{\mathcal{A}_{X}}$ stands for the derived tensor product and $\mathrm{RH}$ om ${ }_{\mathcal{A}_{X}}$ stands for the derived local Hom-functor.

Lemma 2.1. If $f: X \rightarrow Y$ is a morphism of Azumaya varieties, $F \in \mathcal{D}^{-}(X), G \in \mathcal{D}^{+}(Y)$ and $\operatorname{supp}(F)$ is projective over $Y$, then $f_{*} \mathrm{R} \mathcal{H}$ om $_{\mathcal{A}_{X}}\left(F, f^{!} G\right) \cong \mathrm{R} \mathcal{H} o m_{\mathcal{A}_{Y}}\left(f_{*} F, G\right)$.

Proof: Let $Z$ be a scheme-theoretical support of $F$ and let $i: Z \rightarrow X$ denote the corresponding closed embedding, so that $F=i_{*} F^{\prime}$, where $F^{\prime} \in \mathcal{D}^{-}(Z)$. Then both $i$ and $f \circ i: Z \rightarrow Y$ are projective. Using the functoriality of the twisted pullback and the usual duality theorem for $i$ and $f \circ i$ (see [H] and lemma D.34) we deduce

$$
\begin{aligned}
f_{*} \mathrm{RHom}_{\mathcal{A}_{X}}\left(F, f^{!} G\right)=f_{*} \mathrm{RH}^{\prime} \operatorname{mom}_{\mathcal{A}_{X}}\left(i_{*} F^{\prime}, f^{!} G\right) \cong f_{*} i_{*} \mathrm{RH}^{\prime} m_{\mathcal{A}_{X \mid Z}}\left(F^{\prime}, i^{!} f^{!} G\right) \cong \\
\cong(f \circ i)_{*} \mathrm{RHom}_{\mathcal{A}_{X \mid Z}}\left(F^{\prime},(f \circ i)^{!} G\right) \cong \mathrm{RH} \operatorname{mom}_{\mathcal{A}_{Y}}\left((f \circ i)_{*} F^{\prime}, G\right) \cong \mathrm{R} \mathcal{H} o m_{\mathcal{A}_{Y}}\left(f_{*} F, G\right) .
\end{aligned}
$$

Let $X_{1}, X_{2}$ be Azumaya varieties and let $p_{i}: X_{1} \times X_{2} \rightarrow X_{i}$ denote the projections. Take any $K \in \mathcal{D}_{q c}^{-}\left(X_{1} \times X_{2}, \mathcal{A}_{X_{1}}^{\mathrm{opp}} \otimes \mathcal{A}_{X_{2}}\right)$ and define functors

$$
\Phi_{K}\left(F_{1}\right):=p_{2 *}\left(p_{1 \circ}^{*} F_{1} \otimes_{\mathcal{A}_{X_{1}}} K\right), \quad \Phi_{K}^{!}\left(F_{2}\right):=p_{1 *} \mathrm{RH} \mathrm{om}_{\mathcal{A}_{X_{2}}}\left(K, p_{2 \circ} F_{2}\right) .
$$

Then $\Phi_{K}$ is an exact functor $\mathcal{D}_{q c}^{-}\left(X_{1}\right) \rightarrow \mathcal{D}_{q c}^{-}\left(X_{2}\right)$ and $\Phi_{K}^{!}$is an exact functor $\mathcal{D}_{q c}^{+}\left(X_{2}\right) \rightarrow \mathcal{D}_{q c}^{+}\left(X_{1}\right)$. We call $\Phi_{K}$ the kernel functor with kernel $K$, and $\Phi_{K}^{!}$the kernel functor of the second type with kernel $K$.

Lemma 2.2. If $X_{1}$ is smooth and $K \in \mathcal{D}^{\text {perf }}\left(X_{1} \times X_{2}\right)$ then the functor $\Phi_{K}^{!}$is isomorphic to the usual kernel functor with kernel RHom $m_{\mathcal{A}_{X_{2}}}\left(K, \omega_{X_{1}} \otimes \mathcal{A}_{X_{2}}\right)\left[\operatorname{dim} X_{1}\right]$.

Proof: Follows from D.7, D.25 and D.26. 
Lemma 2.3. Any morphism of kernels $\phi: K \rightarrow K^{\prime}$ induces natural morphisms of kernel functors $\phi_{*}: \Phi_{K} \rightarrow \Phi_{K^{\prime}}, \phi^{!}: \Phi_{K^{\prime}}^{!} \rightarrow \Phi_{K}^{!}$. If $\phi$ is an isomorphism, then both $\phi_{*}$ and $\phi^{!}$are isomorphisms.

Proof: Evident.

Lemma 2.4. ( $i)$ If $K$ has coherent cohomologies, finite Tor-amplitude over $X_{1}$ and $\operatorname{supp}(K)$ is projective over $X_{2}$ then $\Phi_{K}$ takes $\mathcal{D}^{b}\left(X_{1}\right)$ to $\mathcal{D}^{b}\left(X_{2}\right)$.

(ii) If $K$ has coherent cohomologies, finite Ext-amplitude over $X_{2}$ and $\operatorname{supp}(K)$ is projective over $X_{1}$ then $\Phi_{K}^{!}$takes $\mathcal{D}^{b}\left(X_{2}\right)$ to $\mathcal{D}^{b}\left(X_{1}\right)$.

(iii) If both (i) and (ii) hold then $\Phi_{K}^{!}$is right adjoint to $\Phi_{K}$. Moreover, $\Phi_{K}$ takes $\mathcal{D}^{\text {perf }}\left(X_{1}\right)$ to $\mathcal{D}^{\text {perf }}\left(X_{2}\right)$.

Proof: (i) If $K$ has finite Tor-amplitude over $X_{1}$ then $q_{1 \circ}^{*} F_{1} \otimes_{\mathcal{A}_{X_{1}}} K \cong q_{1}^{*} F_{1} \otimes_{\mathcal{A}_{X_{1}} \otimes \mathcal{A}_{X_{2}}} K$ is bounded for any $F_{1} \in \mathcal{D}^{b}\left(X_{1}\right)$, and its support is projective over $X_{2}$. Therefore $\Phi_{K}\left(F_{1}\right)$ is bounded and has coherent cohomologies.

(ii) If $K$ has finite Ext-amplitude over $X_{2}$ then $\mathrm{RH} \operatorname{Hom}_{\mathcal{A}_{X_{2}}}\left(K, q_{2 \circ}^{!} F_{2}\right) \cong \mathrm{R} \mathcal{H} o m_{\mathcal{A}_{X_{1}}^{\text {opp }} \otimes \mathcal{A}_{X_{2}}}\left(K, q_{2}^{!} F_{2}\right)$ is bounded for any $F_{2} \in \mathcal{D}^{b}\left(X_{2}\right)$, and its support is projective over $X_{1}$. Therefore $\Phi_{K}^{!}\left(F_{2}\right)$ is bounded and has coherent cohomologies.

(iii) If both $(i)$ and (ii) hold then using lemma 2.1 we deduce

$$
\begin{aligned}
& \operatorname{Hom}_{\mathcal{A}_{X_{1}}}\left(F_{1}, \Phi_{K}^{!}\left(F_{2}\right)\right)=\operatorname{Hom}_{\mathcal{A}_{X_{1}}}\left(F_{1}, p_{1 *}\right.\left.\operatorname{RHom}_{\mathcal{A}_{X_{2}}}\left(K, p_{2 \circ}^{!} F_{2}\right)\right) \cong \\
& \cong \operatorname{Hom}_{\mathcal{A}_{X_{1}}}\left(p_{1 \circ}^{*} F_{1}, \operatorname{RH} \operatorname{mom}_{\mathcal{A}_{X_{2}}}\left(K, p_{2 \circ}^{!} F_{2}\right)\right) \cong \operatorname{Hom}_{\mathcal{A}_{X_{2}}}\left(p_{1 \circ}^{*} F_{1} \otimes_{\mathcal{A}_{X_{1}}} K, p_{2 \circ} F_{2}\right) \cong \\
& \\
& \cong \operatorname{Hom}_{\mathcal{A}_{X_{2}}}\left(p_{2 *}\left(p_{1 \circ}^{*} F_{1} \otimes_{\mathcal{A}_{X_{1}}} K\right), F_{2}\right)=\operatorname{Hom}_{\mathcal{A}_{X_{2}}}\left(\Phi_{K}\left(F_{1}\right), F_{2}\right)
\end{aligned}
$$

for all $F_{1} \in \mathcal{D}^{b}\left(X_{1}\right), F_{2} \in \mathcal{D}^{b}\left(X_{2}\right)$. Moreover, the arguments in $(i i)$ show that $\Phi_{K}^{!}$has bounded cohomological amplitude, hence adjointness implies that $\Phi_{K}$ takes perfect complexes to complexes of finite Ext-amplitude, that is to perfect complexes (see D.44).

Lemma 2.5. If $K$ is a perfect complex, $X_{2}$ is smooth and $\operatorname{supp}(K)$ is projective both over $X_{1}$ and over $X_{2}$, then the kernel functor $\Phi_{K}: \mathcal{D}^{b}\left(X_{2}\right) \rightarrow \mathcal{D}^{b}\left(X_{1}\right)$, where

$$
K^{\#}:=\mathrm{RH}_{\mathrm{H}} m_{\mathcal{A}_{X_{1}}}\left(K, \mathcal{A}_{X_{1}} \otimes \omega_{X_{2}}\left[\operatorname{dim} X_{2}\right]\right),
$$

is the left adjoint functor to the kernel functor $\Phi_{K}: \mathcal{D}^{b}\left(X_{1}\right) \rightarrow \mathcal{D}^{b}\left(X_{2}\right)$.

Proof: Indeed, $\operatorname{supp}\left(K^{\#}\right)=\operatorname{supp}(K)$ is projective over $X_{1}$, hence by lemma 2.1 we have

$$
\begin{aligned}
\operatorname{RHom}_{\mathcal{A}_{X_{1}}}\left(\Phi_{K^{\#}}\left(F_{2}\right), F_{1}\right)=\operatorname{RHom}_{\mathcal{A}_{X_{1}}}\left(p_{1 *}\left(p_{2 \circ}^{*} F_{2} \otimes_{\mathcal{A}_{X_{2}}} K^{\#}\right), F_{1}\right) \cong \\
\cong \operatorname{RHom}_{\mathcal{A}_{X_{1}}}\left(p_{2 \circ}^{*} F_{2} \otimes_{\mathcal{A}_{X_{2}}} K^{\#}, p_{1 \circ}^{!} F_{1}\right) \cong \operatorname{RHom}_{\mathcal{A}_{X_{2}}}\left(p_{2 \circ}^{*} F_{2}, p_{1 \circ} ! F_{1} \otimes_{\mathcal{A}_{X_{1}}} K_{\mathcal{A}_{X_{1}}}^{\# *}\right) \cong \\
\cong \operatorname{RHom}_{\mathcal{A}_{X_{2}}}\left(F_{2}, p_{2 *}\left(p_{1 \circ}^{!} F_{1} \otimes_{\mathcal{A}_{X_{1}}} K_{\mathcal{A}_{X_{1}}}^{\# *}\right)\right) .
\end{aligned}
$$

On the other hand, $K_{\mathcal{A}_{X_{1}}}^{\# *} \cong K \otimes_{\mathcal{O}_{X_{1} \times X_{2}}} \omega_{X_{2}}^{-1}\left[-\operatorname{dim} X_{2}\right]$, and $p_{1 \circ}^{!} F_{1} \otimes_{\mathcal{O}_{X_{1} \times X_{2}}} \omega_{X_{2}}^{-1}\left[-\operatorname{dim} X_{2}\right] \cong p_{1 \circ}^{*} F_{1}$, so the RHS equals to $\mathrm{RHom}_{\mathcal{A}_{X_{2}}}\left(F_{2}, \Phi_{K}\left(F_{1}\right)\right)$.

Consider kernels $K_{12} \in \mathcal{D}^{-}\left(X_{1} \times X_{2}, \mathcal{A}_{X_{1}}^{\text {opp }} \otimes \mathcal{A}_{X_{2}}\right), K_{23} \in \mathcal{D}^{-}\left(X_{2} \times X_{3}, \mathcal{A}_{X_{2}}^{\text {opp }} \otimes \mathcal{A}_{X_{3}}\right)$. Denote by $p_{i j}: X_{1} \times X_{2} \times X_{3} \rightarrow X_{i} \times X_{j}$ the projections. We define the convolution of kernels as follows

$$
K_{23} \circ K_{12}:=p_{13 *}\left(p_{12 \circ}^{*} K_{12} \otimes_{\mathcal{A}_{X_{2}}} p_{23 \circ}^{*} K_{23}\right),
$$

Similarly, if $K_{12} \in \mathcal{D}^{b}\left(X_{1} \times X_{2}, \mathcal{A}_{X_{1}}^{\mathrm{opp}} \otimes \mathcal{A}_{X_{2}}\right)$ and $K_{32} \in \mathcal{D}^{-}\left(X_{3} \times X_{2}, \mathcal{A}_{X_{2}} \otimes \mathcal{A}_{X_{3}}^{\mathrm{opp}}\right)$ we define the convolution of the second type of kernels:

$$
K_{32} \star K_{12}:=p_{13 *} \text { RHom }_{\mathcal{A}_{X_{2}}}\left(p_{23 \circ}^{*} K_{32}, p_{12 \circ}^{!} K_{12}\right) .
$$


Lemma 2.6. For $K_{12} \in \mathcal{D}^{-}\left(X_{1} \times X_{2}\right), K_{23} \in \mathcal{D}^{-}\left(X_{2} \times X_{3}\right), K_{32} \in \mathcal{D}^{-}\left(X_{3} \times X_{2}\right)$ we have

(i) $\Phi_{K_{23}} \circ \Phi_{K_{12}}=\Phi_{K_{23} \circ K_{12}}$,

(ii) $\Phi_{K_{12}}^{!} \circ \Phi_{K_{23}}^{!}=\Phi_{K_{23} \circ K_{12}}^{!}, \quad$ if $\operatorname{supp}\left(p_{12 \circ}^{*} K_{12} \otimes_{\mathcal{A}_{X_{2}}} p_{23 \circ}^{*} K_{23}\right)$ is projective over $X_{1} \times X_{3}$;

(iii) $\left(\Phi_{K_{32}}^{!} \circ \Phi_{K_{12}}\right)_{\mid \mathcal{D}^{\text {perf }}\left(X_{1}\right)}=\Phi_{K_{32} \star K_{12}}, \quad$ if $K_{12} \in \mathcal{D}^{b}\left(X_{1} \times X_{2}\right)$.

Proof: The first is standard. For the second we have

$$
\begin{aligned}
& \left(\Phi_{K_{12}}^{!} \circ \Phi_{K_{23}}^{!}\right)\left(F_{3}\right)=p_{1 *} \mathrm{RH}^{\circ} m_{\mathcal{A}_{X_{2}}}\left(K_{12}, p_{2 \circ} p_{2 *} \mathrm{RH}^{\circ} \mathrm{m}_{\mathcal{A}_{X_{3}}}\left(K_{23}, p_{3 \circ} F_{3}\right)\right) \cong \\
& \cong p_{1 *} \mathrm{RH}^{\circ o m_{\mathcal{A}_{X_{2}}}}\left(K_{12}, p_{12 *} p_{23 \circ} \mathrm{RH} \mathrm{Rm}_{\mathcal{A}_{X_{3}}}\left(K_{23}, p_{3 \circ} F_{3}\right)\right) \cong \\
& \cong p_{1 *} p_{12 *} \mathrm{RH} \mathcal{H}_{\mathcal{A}_{X_{2}}}\left(p_{12 \circ}^{*} K_{12}, \mathrm{RH} \operatorname{Hom}_{\mathcal{A}_{X_{3}}}\left(p_{23 \circ}^{*} K_{23}, p_{23 \circ} p_{3 \circ}^{!} F_{3}\right)\right) \cong
\end{aligned}
$$

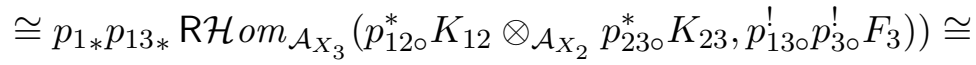

$$
\begin{aligned}
& \left.\cong p_{1 *} \operatorname{RH} \operatorname{cm}_{\mathcal{A}_{X_{3}}}\left(p_{13 *}\left(p_{12 \circ}^{*} K_{12} \otimes_{\mathcal{A}_{X_{2}}} p_{23 \circ}^{*} K_{23}\right), p_{3 \circ}^{!} F_{3}\right)\right)=\Phi_{K_{23} \circ K_{12}}^{!}\left(F_{3}\right) \text {. }
\end{aligned}
$$

Similarly, for the third, if $F_{1}$ is a perfect complex then we have

$$
\begin{aligned}
& \left(\Phi_{K_{32}}^{!} \circ \Phi_{K_{12}}\right)\left(F_{1}\right)=p_{3 *} \mathrm{RH} \mathcal{H}_{\mathcal{A}_{X_{2}}}\left(K_{32}, p_{2 \circ}^{!} p_{2 *}\left(p_{1 \circ}^{*} F_{1} \otimes_{\mathcal{A}_{X_{1}}} K_{12}\right)\right) \cong \\
& \cong p_{3 *} \mathrm{RH}^{\circ} m_{\mathcal{A}_{X_{2}}}\left(K_{32}, p_{23 *} p_{12 \circ}^{!}\left(p_{1 \circ}^{*} F_{1} \otimes_{\mathcal{A}_{X_{1}}} K_{12}\right)\right) \cong \\
& \left.\cong p_{3 *} p_{23 *} \mathrm{RH} \mathrm{om}_{\mathcal{A}_{X_{2}}}\left(p_{23 \circ}^{*} K_{32}, p_{12 \circ}^{*} p_{1 \circ}^{*} F_{1} \otimes_{\mathcal{A}_{X_{1}}} p_{12 \circ}^{!} K_{12}\right)\right) \cong \\
& \left.\cong p_{3 *} p_{13 *} \mathrm{RH}_{\mathrm{H}} m_{\mathcal{A}_{X_{2}}}\left(p_{23 \circ}^{*} K_{32}, p_{13 \circ}^{*} p_{1 \circ}^{*} F_{1} \otimes_{\mathcal{A}_{X_{1}}} p_{12 \circ}^{!} K_{12}\right)\right) \cong \\
& \cong p_{3 *}\left(p_{1 \circ}^{*} F_{1} \otimes_{\mathcal{A}_{X_{1}}} p_{13 *} \mathrm{RH} \mathcal{H}_{\mathcal{A}_{X_{2}}}\left(p_{23 \circ}^{*} K_{32}, p_{12 \circ}^{!} K_{12}\right)\right) \cong \\
& \left.\cong p_{3 *}\left(p_{1 \circ}^{*} F_{1} \otimes_{\mathcal{A}_{X_{1}}}\left(K_{32} \star K_{12}\right)\right)\right)=\Phi_{K_{32} \star K_{12}}\left(F_{1}\right) \text {. }
\end{aligned}
$$

Assume that $\Phi_{1}, \Phi_{2}, \Phi_{3}: \mathcal{D} \rightarrow \mathcal{D}^{\prime}$ are exact functors between triangulated categories, and $\alpha: \Phi_{1} \rightarrow \Phi_{2}$, $\beta: \Phi_{2} \rightarrow \Phi_{3}, \gamma: \Phi_{3} \rightarrow \Phi_{1}[1]$ are morphisms of functors. We say that

$$
\Phi_{1} \stackrel{\alpha}{\longrightarrow} \Phi_{2} \stackrel{\beta}{\longrightarrow} \Phi_{3} \stackrel{\gamma}{\longrightarrow} \Phi_{1}[1]
$$

is an exact triangle of functors, if for any object $F \in \mathcal{D}$ the triangle

$$
\Phi_{1}(F) \stackrel{\alpha(F)}{\longrightarrow} \Phi_{2}(F) \stackrel{\beta(F)}{\longrightarrow} \Phi_{3}(F) \stackrel{\gamma(F)}{\longrightarrow} \Phi_{1}(F)[1]
$$

is exact in $\mathcal{D}^{\prime}$.

Lemma 2.7. If $K_{1} \stackrel{\alpha}{\longrightarrow} K_{2} \stackrel{\beta}{\longrightarrow} K_{3} \stackrel{\gamma}{\longrightarrow} K_{1}[1]$ is an exact triangle in $\mathcal{D}^{-}(X \times Y)$ then we have the following exact triangles of functors

$$
\begin{gathered}
\Phi_{K_{1}} \stackrel{\alpha_{*}}{\longrightarrow} \Phi_{K_{2}} \stackrel{\beta_{*}}{\longrightarrow} \Phi_{K_{3}} \stackrel{\gamma_{*}}{\longrightarrow} \Phi_{K_{1}}[1] \\
\Phi_{K_{3}}^{!} \stackrel{\beta^{!}}{\longrightarrow} \Phi_{K_{2}}^{1} \stackrel{\alpha^{!}}{\longrightarrow} \Phi_{K_{1}}^{!} \stackrel{\gamma^{!}}{\longrightarrow} \Phi_{K_{3}}^{!}[1]
\end{gathered}
$$

Proof: Evident.

Lemma 2.8. Let $\alpha: X \rightarrow Y$ be a finite morphism.

a) If $\mathcal{D}$ is a category and $\Phi: \mathcal{D} \rightarrow \mathcal{D}(X)$ is a functor such that $\alpha_{*} \circ \Phi=0$ then $\Phi=0$.

b) If $X^{\prime}$ is another variety, $K, K^{\prime} \in \mathcal{D}^{-}\left(X^{\prime} \times X\right)$ are kernels and $\phi: K \rightarrow K^{\prime}$ is a morphism, such that morphism of functors $\alpha_{*} \circ \Phi_{K} \stackrel{\alpha_{*}\left(\phi_{*}\right)}{\longrightarrow} \alpha_{*} \circ \Phi_{K^{\prime}}$ is an isomorphism, then $\phi_{*}: \Phi_{K} \rightarrow \Phi_{K^{\prime}}$ is an isomorphism. 
Proof: $a$ ) Since $\alpha$ is finite we have $\mathcal{H}^{i}\left(\alpha_{*} G\right) \cong \alpha_{*} \mathcal{H}^{i}(G)$ for any $G \in \mathcal{D}(X)$. Therefore $\alpha_{*}(G)=0$ implies $\alpha_{*}\left(\mathcal{H}^{i}(G)\right)=0$ for all $i$, hence $G=0$ in $\mathcal{D}(X)$. In particular, $\alpha_{*} \Phi(F)=0$ implies $\Phi(F)=0$ for all $F \in \mathcal{D}$.

b) Let $K^{\prime \prime}$ be the cone of $\phi: K \rightarrow K^{\prime}$ in $\mathcal{D}^{-}\left(X^{\prime} \times X\right)$. Then we have an exact triangle of functors $\Phi_{K} \rightarrow \Phi_{K^{\prime}} \rightarrow \Phi_{K^{\prime \prime}}$ and it follows that $\alpha_{*} \circ \Phi_{K^{\prime \prime}}=0$. Therefore, $\Phi_{K^{\prime \prime}}=0$ by part (a), hence $\phi_{*}$ is an isomorphism.

\subsection{Perfect spanning classes.}

Definition 2.9. A class of objects $\mathcal{F} \subset \mathcal{D}\left(X, \mathcal{A}_{X}\right)$ is called a perfect spanning class for $\left(X, \mathcal{A}_{X}\right)$, if for any point $x \in X$ there exists an object $0 \neq F_{x} \in \mathcal{F}$ such that

(1) $F_{x}$ is a perfect complex;

(2) $\mathcal{H}^{p}\left(F_{x}\right)$ is supported set theoretically at $x$ for all $p$;

(3) if $p_{0}=\max \left\{p \mid \mathcal{H}^{p}\left(F_{x}\right) \neq 0\right\}$ then $\mathcal{H}^{p_{0}}\left(F_{x}\right) \cong \mathcal{A}_{X} \otimes_{\mathcal{O}_{X}} \mathcal{O}_{x}$

Lemma 2.10. Any Azumaya variety admits a perfect spanning class.

Proof: Choose a closed embedding $i: X \rightarrow Y$ with smooth $Y$ and take $F_{x}:=\mathcal{A}_{X} \otimes_{\mathcal{O}_{X}} i^{*} i_{*} \mathcal{O}_{x}$.

Lemma 2.11. If $\mathcal{F}$ is a perfect spanning class for $X$ and $\mathcal{G}$ is a perfect spanning class for $Y$ then $\mathcal{F} \otimes \mathcal{G}$ is a perfect spanning class for $X \times Y$.

Proof: Evident.

Lemma 2.12. Assume that $f: X \rightarrow S$ and $g: Y \rightarrow S$ are morphisms of algebraic varieties. If $\mathcal{F}$ is a perfect spanning class in $\mathcal{D}\left(X, \mathcal{A}_{X}\right)$ and $\mathcal{G}$ is a perfect spanning class in $\mathcal{D}\left(Y, \mathcal{O}_{Y}\right)$ then $p^{*} \mathcal{F} \otimes_{\mathcal{O}_{X \times{ }_{S} Y}} q_{\circ}^{*} \mathcal{G}$ is a perfect spanning class in $\mathcal{D}\left(X \times_{S} Y, p_{\circ}^{*} \mathcal{A}_{X}\right)$, where $p: X \times_{S} Y \rightarrow X$ and $q: X \times_{S} Y \rightarrow Y$ are the projections.

Proof: Evident.

Lemma 2.13. If $K \in \mathcal{D}^{-}\left(X, \mathcal{A}_{X}^{\mathrm{opp}}\right), \mathcal{F}$ is a perfect spanning class for $\left(X, \mathcal{A}_{X}\right)$ and for all $F \in \mathcal{F}$ we have $H^{\bullet}\left(X, F \otimes_{\mathcal{A}_{X}} K\right)=0$, then $K=0$.

Proof: Assume that $K \neq 0$ and let $s$ be the maximal integer such that $\mathcal{H}^{s}(K) \neq 0$. Choose a point $x \in$ supp $\mathcal{H}^{s}(K)$, and take an object $F_{x} \in \mathcal{F}$, corresponding to this point. Let $t$ be the maximal integer such that $\mathcal{H}^{t}\left(F_{x}\right) \neq 0$. Then it is clear that $\mathcal{H}^{p}\left(F_{x} \otimes_{\mathcal{A}_{X}} K\right)$ is supported at $x$ for all $p, \mathcal{H}^{>s+t}\left(F_{x} \otimes_{\mathcal{A}_{X}} K\right)=0$, and

$$
\mathcal{H}^{s+t}\left(F_{x} \otimes_{\mathcal{A}_{X}} K\right) \cong \mathcal{H}^{0}\left(\left(\mathcal{A}_{X} \otimes_{\mathcal{O}_{X}} \mathcal{O}_{x}\right) \otimes_{\mathcal{A}_{X}} \mathcal{H}^{s}(K)\right) \cong \mathcal{H}^{0}\left(\mathcal{H}^{s}(K) \otimes_{\mathcal{O}_{X}} \mathcal{O}_{x}\right) \neq 0 .
$$

It follows that the hypercohomology spectral sequence for $F_{x} \otimes_{\mathcal{A}_{X}} K$

$$
E_{2}^{p, q}=H^{q}\left(X, \mathcal{H}^{p}\left(F_{x} \otimes_{\mathcal{A}_{X}} K\right)\right) \Rightarrow H^{p+q}\left(X, F_{x} \otimes_{\mathcal{A}_{X}} K\right)
$$

degenerates in the second term, and $H^{s+t}\left(X, F_{x} \otimes_{\mathcal{A}_{X}} K\right)=H^{0}\left(X, \mathcal{H}^{s+t}\left(F_{x} \otimes_{\mathcal{A}_{X}} K\right)\right) \neq 0$, which is a contradiction.

Proposition 2.14. If $K \in \mathcal{D}^{-}\left(X \times Y, \mathcal{A}_{X}^{\mathrm{opp}} \otimes \mathcal{A}_{Y}\right)$ and the functor $\Phi_{K}: \mathcal{D}\left(X, \mathcal{A}_{X}\right) \rightarrow \mathcal{D}\left(Y, \mathcal{A}_{Y}\right)$ equals zero on a perfect spanning class $\mathcal{F}$ for $\left(X, \mathcal{A}_{X}\right)$ then $K=0$.

Proof: Choose a perfect spanning class $\mathcal{G}$ for $\left(Y, \mathcal{O}_{Y}\right)$. Then for all $F \in \mathcal{F}, G \in \mathcal{G}$ we have

$$
H^{\bullet}\left(X \times Y,(F \otimes G) \otimes_{\mathcal{A}_{X}} K\right) \cong H^{\bullet}\left(Y, q_{*}\left(\left(p_{\circ}^{*} F \otimes_{\mathcal{A}_{X}} K\right) \otimes_{\mathcal{O}_{X \times Y}} q_{\circ}^{*} G\right)\right) \cong H^{\bullet}\left(Y, \Phi_{K}(F) \otimes_{\mathcal{O}_{Y}} G\right)=0 .
$$

Since $\mathcal{F} \otimes \mathcal{G}$ is a perfect spanning class for $\left(X \times Y, \mathcal{A}_{X}\right)$, we have $K=0$. 
Corollary 2.15. If the morphism of kernel functors $\Phi_{K} \rightarrow \Phi_{K^{\prime}}$ with $K, K^{\prime} \in \mathcal{D}^{-}(X \times Y)$, induced by a morphism of kernels $\phi: K \rightarrow K^{\prime}$ is an isomorphism on a perfect spanning class $\mathcal{F}$ for $X$, then $\varphi$ is an isomorphism.

Proof: Let $K^{\prime \prime}$ be the cone of $\phi: K \rightarrow K^{\prime}$ in $\mathcal{D}^{-}(X \times Y)$. Then we have an exact triangle of functors $\Phi_{K} \rightarrow \Phi_{K^{\prime}} \rightarrow \Phi_{K^{\prime \prime}}$ and it follows that $\Phi_{K^{\prime \prime}}$ equals zero on the perfect spanning class $\mathcal{F}$. Therefore, $K^{\prime \prime}=0$, hence $\phi_{*}$ is an isomorphism.

2.3. Koszul complexes. If $\left(X, \mathcal{A}_{X}\right)$ is an Azumaya variety, $\mathcal{V}$ is a vector bundle on its underlying algebraic variety $X, s \in \Gamma(X, \mathcal{V})$ is a section (possible nonregular) of $\mathcal{V}$, then we denote by $\operatorname{Kosz}_{X}(s)$ the Koszul complex of $s$ considered as an object of the derived category $\mathcal{D}^{b}\left(X, \mathcal{A}_{X}\right)$ :

$$
\operatorname{Kosz}_{X}(s):=\left\{0 \rightarrow \Lambda^{\text {top }} \mathcal{V}^{*} \otimes_{\mathcal{O}_{X}} \mathcal{A}_{X} \stackrel{s}{\longrightarrow} \ldots \stackrel{s}{\longrightarrow} \mathcal{V}^{*} \otimes_{\mathcal{O}_{X}} \mathcal{A}_{X} \stackrel{s}{\longrightarrow} \mathcal{A}_{X} \rightarrow 0\right\}
$$

with $\mathcal{A}_{X}$ placed in degree 0 . Assume that $X$ is Cohen-Macaulay. Recall that a section $s$ is regular if the codimension of the zero locus $Z(s) \subset X$ of $s$ is equal to the rank of $\mathcal{V}$. It is well known that for regular section $s$ we have $\operatorname{Kosz}_{X}(s) \cong i_{*} \mathcal{A}_{Z(s)}$, where $i: Z(s) \rightarrow X$ is the embedding and $\mathcal{A}_{Z(s)}=\mathcal{A}_{X \mid Z(s)}$.

Lemma 2.16. Assume that $X$ is a Cohen-Macaulay variety. Then for any section $s \in \Gamma(X, \mathcal{V})$ we have $\operatorname{Kosz}_{X}(s) \in \mathcal{D}^{\left[\operatorname{codim}_{X} Z(s)-\operatorname{rank} \mathcal{V}, 0\right]}\left(X, \mathcal{A}_{X}\right)$. Moreover, $\operatorname{Kosz}_{X}(s)$ is supported scheme-theoretically on a infinitesimal neighborhood of $Z(s) \subset X$ and $\mathcal{H}^{0}\left(\operatorname{Kosz}_{X}(s)\right) \cong i_{*} \mathcal{A}_{Z(s)}$.

Proof: The first part of the claim is local. Locally, we can decompose $\mathcal{V}=\mathcal{V}^{\prime} \oplus \mathcal{V}^{\prime \prime}$, so that the first component of $s=\left(s^{\prime}, s^{\prime \prime}\right)$ is regular and rank $\mathcal{V}^{\prime}=\operatorname{codim}_{X} Z(s)$. Then denoting by $i^{\prime}: Z\left(s^{\prime}\right) \rightarrow X$ the embedding, we get

$$
\operatorname{Kosz}_{X}(s) \cong \operatorname{Kosz}_{X}\left(s^{\prime}\right) \otimes_{\mathcal{A}_{X}} \operatorname{Kosz}_{X}\left(s^{\prime \prime}\right) \cong i_{*}^{\prime} \mathcal{A}_{Z\left(s^{\prime}\right)} \otimes_{\mathcal{A}_{X}} \operatorname{Kosz}_{X}\left(s^{\prime \prime}\right) \cong i_{*}^{\prime} \operatorname{Kosz}_{Z\left(s^{\prime}\right)}\left(i^{\prime *} s^{\prime \prime}\right),
$$

and it remains to note that we have $\operatorname{Kosz}_{Z\left(s^{\prime}\right)}\left(i^{\prime *} s^{\prime \prime}\right) \in \mathcal{D}^{\left[-\operatorname{rank} \mathcal{V}^{\prime \prime}, 0\right]}\left(Z\left(s^{\prime}\right), \mathcal{A}_{Z\left(s^{\prime}\right)}\right)$ by definition, and $\operatorname{rank} \mathcal{V}^{\prime \prime}=\operatorname{rank} \mathcal{V}-\operatorname{rank} \mathcal{V}^{\prime}=\operatorname{rank} \mathcal{V}-\operatorname{codim}_{X} Z(s)$

The second part of the claim is evident since $\operatorname{Kosz}_{X}(s)$ is acyclic on the complement of $Z(s)$, and the third part follows from the definition of the sheaf of ideals of $Z(s)$ as the image of the morphism $\mathcal{V}^{*} \stackrel{s}{\longrightarrow} \mathcal{O}_{X}$.

2.4. Exact cartesian squares. Let $f:\left(X, \mathcal{A}_{X}\right) \rightarrow\left(S, \mathcal{A}_{S}\right)$ and $g:\left(Y, \mathcal{A}_{Y}\right) \rightarrow\left(S, \mathcal{A}_{S}\right)$ be morphisms of Azumaya varieties. Assume that either $f$ or $g$ is strict. In this case there is defined the fiber product $\left(X \times_{S} Y, \mathcal{A}_{X \times{ }_{S} Y}\right)$, see D.37. Consider the corresponding cartesian square

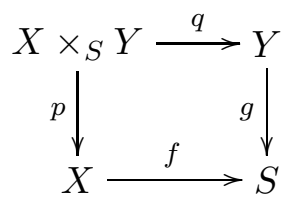

Let $\Gamma_{f}: X \rightarrow X \times S$ and $\Gamma^{g}: Y \rightarrow S \times Y$ denote the graphs of morphisms $f$ and $g$ respectively, and put $K_{f}=\Gamma_{f_{*}} \mathcal{A}_{X}, K^{g}=\Gamma_{*}^{g} \mathcal{A}_{Y}, K(f, g)=\mathcal{A}_{X \times_{S} Y}$ and consider the corresponding kernel functors. Then we have functorial isomorphisms

$$
\Phi_{K_{f}} \cong f_{*}, \quad \Phi_{K^{g}} \cong g^{*}, \quad \Phi_{K(f, g)} \cong q_{*} p^{*} .
$$

Lemma 2.17. We have $K_{f} \circ K^{g} \in \mathcal{D}^{\leq 0}(X \times Y)$ and $\mathcal{H}^{0}\left(K_{f} \circ K^{g}\right) \cong K(f, g)$. 
Proof: Let $p_{X S}: X \times S \times Y \rightarrow X \times S, p_{S Y}: X \times S \times Y \rightarrow S \times Y$ denote the projections, and consider the object $K=p_{X S \circ}^{*} K_{f} \otimes_{\mathcal{A}_{S}} p_{S Y \circ}^{*} K^{g}$. Since the pullback and the tensor product are left exact, we have $K \in \mathcal{D}^{\leq 0}$. Further, it is clear that the cohomologies of $K$ are supported (set-theoretically) on the fiber product $X \times_{S} Y=X \times{ }_{S} S \times{ }_{S} Y \subset X \times S \times Y$ and $\mathcal{H}^{0}(K) \cong \mathcal{A}_{X \times{ }_{S} Y}$. Since the projection $p_{X Y}: X \times S \times Y \rightarrow X \times Y$ restricted to the infinitesimal neighborhood of $X \times_{S} Y$ in $X \times S \times Y$ is finite, we deduce that $K_{f} \circ K^{g}=p_{X Y *} K \in \mathcal{D}^{\leq 0}$, and $\mathcal{H}^{0}\left(K_{f} \circ K^{g}\right) \cong p_{X Y{ }_{*}} \mathcal{A}_{X \times_{S} Y} \cong K(f, g)$.

The canonical morphism of kernels $K_{f} \circ K^{g} \rightarrow \mathcal{H}^{0}\left(K_{f} \circ K^{g}\right)$ induces a functorial morphism $g^{*} f_{*} \rightarrow q_{*} p^{*}$.

Definition 2.18. A cartesian square (1) is called exact cartesian, if the natural morphism of functors $g^{*} f_{*} \rightarrow q_{*} p^{*}$ is an isomorphism.

Proposition 2.19. A cartesian square (1) is exact cartesian, if and only if the canonical morphism $K_{f} \circ K^{g} \rightarrow \mathcal{H}^{0}\left(K_{f} \circ K^{g}\right) \cong K(f, g)$ is an isomorphism.

Proof: The "only if" part follows from corollary 2.15 and the "if" part from lemma 2.3.

Further, corollary 2.15 implies

Corollary 2.20. If the natural morphism of functors $g^{*} f_{*} \rightarrow q_{*} p^{*}$ is an isomorphism on a perfect spanning class $\mathcal{F} \subset \mathcal{D}\left(X, \mathcal{A}_{X}\right)$, then cartesian square (1) is exact cartesian.

Another consequence of proposition 2.19 is

Corollary 2.21. A cartesian square (1) is exact cartesian, if and only if the transposed square is exact cartesian.

Proof: The criterion of proposition 2.19 is symmetric.

Lemma 2.22. A fiber square (1) is exact cartesian, if and only if the underlying square of algebraic varieties is.

Proof: Note that the fiber square of Azumaya varieties was defined only in a situation when one of morphisms $f, g$ is strict. Since the exactness property is symmetric with respect to the transposition of the square, we may assume that $g$ is strict. Then $p$ is also strict, and we have $q_{*} p^{*} \cong q_{*} p_{\circ}^{*}$, and $g^{*} f_{*} \cong g_{\circ}^{*} f_{*}$. It remains to note that the functorial morphism $g_{\circ}^{*} f_{*} \rightarrow q_{*} p_{\circ}^{*}$ is obtained from the functorial morphism $g^{*} f_{*} \rightarrow q_{*} p^{*}$ by forgetting the $\mathcal{A}_{Y}$-module structure.

Corollary 2.23. If either $f$ or $g$ is flat then cartesian square (1) is exact cartesian.

Proof: Since the exactness property is symmetric with respect to the transposition of the square, we may assume that $g$ is flat. Then the underlying square is exact by $[\mathrm{H}], \mathrm{II}$, proposition 5.12 .

Proposition 2.24. If diagram (1) is exact cartesian then $f^{!} g_{*} \cong p_{*} q^{!}$.

Proof: Note that $f^{!} \cong \Phi_{K_{f}}^{!}, g_{*} \cong \Phi_{K^{g}}^{!}, p_{*} q^{!} \cong \Phi_{K(f, g)}^{!}$and apply lemma 2.6 (ii).

Lemma 2.25. Assume that the right square in the following diagram is exact cartesian

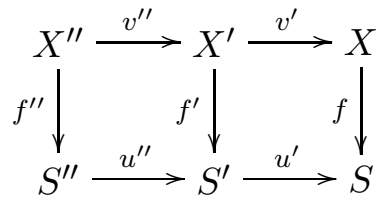

and either $f$ or both $u^{\prime}$ and $u^{\prime \prime}$ are strict. Then the ambient square is exact cartesian if and only if the left square is. 
Proof: Assume that the ambient square is exact and $f$ is strict. We choose perfect spanning classes $\mathcal{F} \subset \mathcal{D}\left(X, \mathcal{O}_{X}\right), \mathcal{G} \subset \mathcal{D}\left(S^{\prime}, \mathcal{A}_{S^{\prime}}\right)$. Then for all $F \in \mathcal{F}, G \in \mathcal{G}$ we have

$$
\begin{aligned}
& f_{*}^{\prime \prime} v^{\prime \prime *}\left(v_{\circ}^{\prime *} F \otimes_{\mathcal{O}} f^{\prime *} G\right) \cong f_{*}^{\prime \prime}\left(v_{\circ}^{\prime \prime *} v_{\circ}^{\prime *} F \otimes_{\mathcal{O}} v^{\prime \prime *} f^{\prime *} G\right) \cong \\
& \cong f_{*}^{\prime \prime}\left(\left(v^{\prime} v^{\prime \prime}\right)_{\circ}^{*} F \otimes_{\mathcal{O}} f^{\prime \prime *} u^{\prime \prime *} G\right) \cong f_{*}^{\prime \prime}\left(v^{\prime} v^{\prime \prime}\right)_{\circ}^{*} F \otimes_{\mathcal{O}} u^{\prime \prime *} G \cong\left(u^{\prime} u^{\prime \prime}\right)_{\circ}^{*} f_{*} F \otimes_{\mathcal{O}} u^{\prime \prime *} G \cong u^{\prime \prime *}\left(u_{\circ}^{\prime *} f_{*} F \otimes_{\mathcal{O}} G\right) \cong \\
& \cong u^{\prime \prime *}\left(f^{\prime}{ }_{*}^{\prime} v_{\circ}^{\prime *} F \otimes_{\mathcal{O}} G\right) \cong u^{\prime \prime *}{f^{\prime}}_{*}\left(v_{\circ}^{\prime *} F \otimes_{\mathcal{O}} f^{\prime *} G\right) .
\end{aligned}
$$

It remains to note that objects of the type $v_{\circ}^{\prime *} F \otimes_{\mathcal{O}} f^{\prime *} G$ form a perfect spanning class in the derived category of $X^{\prime}=X \times{ }_{S} S^{\prime}$, and apply corollary 2.20 .

Assume that the ambient square is exact and both $u^{\prime}$ and $u^{\prime \prime}$ are strict. We choose perfect spanning classes $\mathcal{F} \subset \mathcal{D}\left(X, \mathcal{A}_{X}\right), \mathcal{G} \subset \mathcal{D}\left(S^{\prime}, \mathcal{O}_{S^{\prime}}\right)$. Then for all $F \in \mathcal{F}, G \in \mathcal{G}$ we have

$$
\begin{aligned}
& f_{*}^{\prime \prime} v^{\prime \prime *}\left(v^{\prime *} F \otimes_{\mathcal{O}} f_{\circ}^{\prime *} G\right) \cong f_{*}^{\prime \prime}\left(v^{\prime \prime *} v^{\prime *} F \otimes_{\mathcal{O}} v_{\circ}^{\prime \prime *} f_{\circ}^{\prime *} G\right) \cong \\
& \cong f_{*}^{\prime \prime}\left(\left(v^{\prime} v^{\prime \prime}\right)^{*} F \otimes_{\mathcal{O}} f_{\circ}^{\prime \prime *} u_{\circ}^{\prime \prime *} G\right) \cong f_{*}^{\prime \prime}\left(v^{\prime} v^{\prime \prime}\right)^{*} F \otimes_{\mathcal{O}} u_{\circ}^{\prime \prime *} G \cong\left(u^{\prime} u^{\prime \prime}\right)^{*} f_{*} F \otimes_{\mathcal{O}} u_{\circ}^{\prime \prime *} G \cong u^{\prime \prime *}\left(u^{\prime *} f_{*} F \otimes_{\mathcal{O}} G\right) \cong \\
& \cong u^{\prime \prime *}\left(f^{\prime}{ }_{*} v^{* *} F \otimes_{\mathcal{O}} G\right) \cong u^{\prime \prime *} f^{\prime}{ }_{*}\left(v^{\prime *} F \otimes_{\mathcal{O}} f_{\circ}^{\prime *} G\right) .
\end{aligned}
$$

It remains to note that objects of the type $v^{\prime *} F \otimes_{\mathcal{O}} f_{\circ}^{\prime *} G$ form a perfect spanning class in the derived category of $X^{\prime}=X \times_{S} S^{\prime}$, and apply corollary 2.20 .

On the other hand, if the left square is exact, then

$$
f_{*}^{\prime \prime}\left(v^{\prime} v^{\prime \prime}\right)^{*} F \cong f_{*}^{\prime \prime} v^{\prime \prime *}\left(v^{\prime *} F\right) \cong u^{\prime \prime *}\left(f_{*}^{\prime} v^{\prime *}\right) F \cong u^{\prime \prime *} u^{\prime *} f_{*} F \cong\left(u^{\prime} u^{\prime \prime}\right)^{*} f_{*} F
$$

for any $F \in \mathcal{D}^{b}\left(X, \mathcal{A}_{X}\right)$, hence the ambient square is exact.

Lemma 2.26. If $g$ is finite and the canonical morphism

$$
f^{*} g_{*} \mathcal{A}_{Y} \rightarrow p_{*} q^{*} \mathcal{A}_{Y} \cong p_{*} \mathcal{A}_{X \times{ }_{S} Y}
$$

is an isomorphism, then square (1) is exact cartesian.

Proof: Note that

$$
g_{*} g^{*} f_{*}(F) \cong f_{*}(F) \otimes_{\mathcal{A}_{S}} g_{*} \mathcal{A}_{Y} \cong f_{*}\left(F \otimes_{\mathcal{A}_{X}} f^{*} g_{*} \mathcal{A}_{Y}\right) \cong f_{*}\left(F \otimes_{\mathcal{A}_{X}} p_{*} \mathcal{A}_{X \times_{S} Y}\right) \cong f_{*} p_{*} p^{*}(F) \cong g_{*} q_{*} p^{*}(F),
$$

and the resulting isomorphism coincides with the morphism, obtained by application of the pushforward functor $g_{*}$ to the functorial morphism $g^{*} f_{*} \rightarrow q_{*} p^{*}$. Since the latter is induced by a morphism of kernels $K_{f} \circ K^{g} \rightarrow K(f, g)$ it remains to apply lemma $\left.2.8 \mathrm{~b}\right)$.

Corollary 2.27. Assume that $g$ is a strict closed embedding, $Y \subset S$ is a locally complete intersection, and both $S$ and $X$ are Cohen-Macaulay. If $\operatorname{codim}_{X}\left(X \times_{S} Y\right)=\operatorname{codim}_{S} Y$, then square (1) is exact cartesian.

Proof: By lemma 2.26 it suffices to check that the canonical homomorphism $f^{*} g_{*} \mathcal{A}_{Y} \rightarrow p_{*} \mathcal{A}_{X \times{ }_{S} Y}$ is an isomorphism. The claim is local with respect to $S$, so we may assume that $Y$ is a zero locus of a regular section $s$ of a vector bundle $\mathcal{V}$ on $S$ of $\operatorname{rank} \operatorname{rank} \mathcal{V}=\operatorname{codim}_{S} Y$. Then $g_{*} \mathcal{A}_{Y} \cong \operatorname{Kosz}_{S}(s)$, hence $f^{*} g_{*} \mathcal{A}_{Y} \cong f^{*} \operatorname{Kosz}_{S}(s) \cong \operatorname{Kosz}_{X}\left(f^{*} s\right)$. It is clear that the zero locus of $f^{*} s$ on $X$ is the fiber product $X \times_{S} Y$, but $\operatorname{codim}_{X}\left(X \times_{S} Y\right)=\operatorname{codim}_{S} Y=\operatorname{rank} \mathcal{V}=\operatorname{rank} f^{*} \mathcal{V}$ which implies that the section $f^{*} s$ is regular and we have an isomorphism $\operatorname{Kosz}_{X}\left(f^{*} s\right) \cong p_{*} \mathcal{A}_{X \times{ }_{S} Y}$.

Corollary 2.28. Consider exact cartesian square (1) and let $\mathcal{U}, \mathcal{V}$, and $\mathcal{W}$ be vector bundles on $S, X$, and $Y$ respectively, with sections $u \in \Gamma(S, \mathcal{U}), v \in \Gamma(X, \mathcal{V})$, and $w \in \Gamma(Y, \mathcal{W})$. Assume that the sections

$$
\begin{aligned}
p^{*} f^{*} u+p^{*} v+q^{*} w & \in \Gamma\left(X \times_{S} Y, p^{*} f^{*} \mathcal{U} \oplus p^{*} \mathcal{V} \oplus q^{*} \mathcal{W}\right) & g^{*} u+w & \in \Gamma\left(Y, g^{*} \mathcal{U} \oplus \mathcal{W}\right) \\
f^{*} u+v & \in \Gamma\left(X, f^{*} \mathcal{U} \oplus \mathcal{V}\right) & u & \in \Gamma(S, \mathcal{U})
\end{aligned}
$$


are regular. Denote by $S_{u} \subset S, X_{u, v} \subset X, Y_{u, w} \subset Y$ and $Z_{u, v, w} \subset Z=X \times_{S} Y$ the corresponding zero loci. Then the square

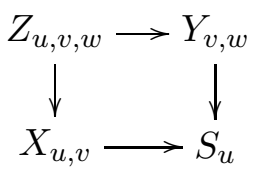

is exact cartesian.

Proof: Consider the diagrams

1)

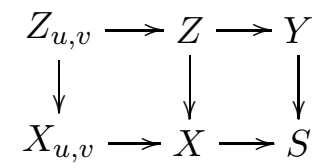

2)

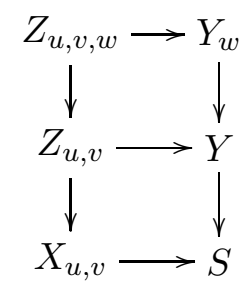

3)

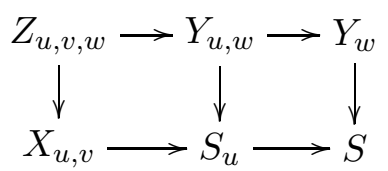

In the first diagram the right square is exact by assumption and the left square is exact by corollary 2.27 . Hence the ambient square is exact by the "if" part of lemma 2.25. Thus the bottom square in the second diagram is exact. On the other hand, its upper square is exact by corollary 2.27. Hence its ambient square is exact by the "if" part of lemma 2.25. Thus the ambient square in the third diagram is exact. On the other hand, its right square is exact by corollary 2.27 . Hence its left square is exact by the "only if" part of lemma 2.25.

Definition 2.29. A strict morphism $\phi: T \rightarrow S$ is called faithful with respect to a morphism $f: X \rightarrow S$ if the cartesian square

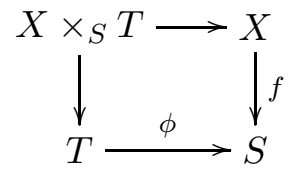

is exact.

Lemma 2.30. A strict and flat base change is faithful with respect to any morphism.

Proof: Use lemma 2.23 .

Corollary 2.31. Let $T \rightarrow S$ be a strict base change faithful with respect to a morphism $f: X \rightarrow S$. If $T \rightarrow S^{\prime} \rightarrow S$ is a factorization of $T \rightarrow S$ such that $T \rightarrow S^{\prime}$ is strict and finite and $S^{\prime} \rightarrow S$ is strict and smooth, then $S^{\prime} \rightarrow S$ is faithful with respect to $f$ and $T \rightarrow S^{\prime}$ is faithful with respect to $f^{\prime}: X \times_{S} S^{\prime} \rightarrow S^{\prime}$.

Proof: The smooth base change is faithful by lemma 2.30 and the finite base change is faithful by the "only if" part of the lemma 2.25 .

Lemma 2.32. If square (1) is exact cartesian, $g$ has finite Tor-dimension and strict, and $f$ is projective then there exists a canonical isomorphism of functors

$$
p^{*} f^{!} \cong q^{!} g^{*}: \mathcal{D}^{+}(S) \rightarrow \mathcal{D}^{+}\left(X \times{ }_{S} Y\right) .
$$

Proof: First of all, we note that

$$
\operatorname{Hom}\left(p^{*} f^{!}(F), q^{!} g^{*}(F)\right) \cong \operatorname{Hom}\left(q_{*} p^{*} f^{!}(F), g^{*}(F)\right) \cong \operatorname{Hom}\left(g^{*} f_{*} f^{!}(F), g^{*}(F)\right),
$$

hence the canonical adjunction morphism $f_{*} f^{!} \rightarrow$ id induces a functorial morphism $p^{*} f^{!} \rightarrow q^{!} g^{*}$. Let us show that it is an isomorphism. 
Decomposing $f$ as $X \stackrel{f_{1}}{\longrightarrow} S^{\prime} \stackrel{f_{2}}{\longrightarrow} S$, where $f_{1}$ is finite and $f_{2}$ is strict and smooth, and applying lemma 2.25 we reduce the claim of the lemma to two cases, the case of strict and smooth $f$, and the case of finite $f$, which can be treated separately.

If $f$ is strict and smooth then $q$ is also strict and smooth and

$$
f^{!}(F) \cong f^{*} F \otimes_{\mathcal{O}_{X}} \omega_{X / S}[\operatorname{dim} X-\operatorname{dim} S], \quad q^{!}(F) \cong q^{*} F \otimes_{\mathcal{O}_{X \times{ }_{S} Y}} \omega_{X \times_{S} Y / Y}\left[\operatorname{dim} X \times_{S} Y-\operatorname{dim} Y\right] .
$$

It remains to note that $\omega_{X \times{ }_{S} Y / Y} \cong p_{\circ}^{*} \omega_{X / S}$ and $\operatorname{dim} X \times_{S} Y=\operatorname{dim} X+\operatorname{dim} Y-\operatorname{dim} S$ since $f$ is smooth, hence

$$
\begin{aligned}
&\left.p^{*}\left(f^{!} F\right) \cong p^{*}\left(f^{*} F \otimes_{\mathcal{O}_{X}} \omega_{X / S}[\operatorname{dim} X-\operatorname{dim} S]\right) \cong p^{*} f^{*} F \otimes_{\mathcal{O}_{X \times{ }_{S} Y}} p_{\circ}^{*} \omega_{X / S}[\operatorname{dim} X-\operatorname{dim} S]\right) \cong \\
& \cong q^{*} g^{*} F \otimes_{\mathcal{O}_{X \times_{S} Y}} \omega_{X \times_{S} Y / Y}\left[\operatorname{dim} X \times_{S} Y-\operatorname{dim} Y\right] \cong q^{!}\left(g^{*} F\right) .
\end{aligned}
$$

Now, assume that $f$ is finite. Then $q$ is also finite and

$$
\begin{aligned}
& q_{*} q^{!} g^{*}(F) \cong \mathrm{R} \mathcal{H} o m\left(q_{*} \mathcal{A}_{X \times{ }_{S} Y}, g^{*} F\right) \cong \mathrm{R} \mathcal{H} o m\left(q_{*} p^{*} \mathcal{A}_{X}, g^{*} F\right) \cong \\
& \cong \mathrm{RHom}\left(g^{*} f_{*} \mathcal{A}_{X}, g^{*} F\right) \cong g^{*} \operatorname{RHom}\left(f_{*} \mathcal{A}_{X}, F\right) \cong g^{*} f_{*} f^{!}(F) \cong q_{*} p^{*} f^{!}(F),
\end{aligned}
$$

and it remains to apply lemma $2.8 \mathrm{~b}$ ).

2.5. Derived categories over a base. Consider a pair of Azumaya varieties $\left(X, \mathcal{A}_{X}\right)$ and $\left(Y, \mathcal{A}_{Y}\right)$ over the same smooth algebraic variety $S$. In other words, we have a pair of morphisms $f:\left(X, \mathcal{A}_{X}\right) \rightarrow\left(S, \mathcal{O}_{S}\right)$ and $g:\left(Y, \mathcal{A}_{Y}\right) \rightarrow\left(S, \mathcal{O}_{S}\right)$.

A functor $\Phi: \mathcal{D}\left(X, \mathcal{A}_{X}\right) \rightarrow \mathcal{D}\left(Y, \mathcal{A}_{Y}\right)$ is called $S$-linear if for all $F \in \mathcal{D}\left(X, \mathcal{A}_{X}\right), G \in \mathcal{D}^{b}\left(S, \mathcal{O}_{S}\right)$ there are given bifucntorial isomorphisms

$$
\Phi\left(f_{\circ}^{*} G \otimes_{\mathcal{O}_{X}} F\right) \cong g_{\circ}^{*} G \otimes_{\mathcal{O}_{Y}} \Phi(F) .
$$

Note that since $S$ is smooth any object $G \in \mathcal{D}^{b}\left(S, \mathcal{O}_{S}\right)$ is a perfect complex.

Lemma 2.33. If $\Phi$ is $S$-linear and admits a right adjoint functor $\Phi^{!}$then $\Phi^{!}$is also $S$-linear.

Proof: For all $F \in \mathcal{D}\left(X, \mathcal{A}_{X}\right), G \in \mathcal{D}\left(Y, \mathcal{A}_{Y}\right)$ and $H \in \mathcal{D}^{b}(S)$ we have

$$
\begin{aligned}
& \operatorname{RHom}_{\mathcal{A}_{X}}\left(F, \Phi^{!}\left(g_{\circ}^{*} H \otimes_{\mathcal{O}_{Y}} G\right)\right) \cong \operatorname{RHom}_{\mathcal{A}_{Y}}\left(\Phi(F), g_{\circ}^{*} H \otimes_{\mathcal{O}_{Y}} G\right) \cong \operatorname{RHom}_{\mathcal{A}_{Y}}\left(g_{\circ}^{*} H^{*} \otimes_{\mathcal{O}_{Y}} \Phi(F), G\right) \cong \\
& \cong \operatorname{RHom}_{\mathcal{A}_{Y}}\left(\Phi\left(f_{\circ}^{*} H^{*} \otimes_{\mathcal{O}_{X}} F\right), G\right) \cong \operatorname{RHom}_{\mathcal{A}_{X}}\left(f_{\circ}^{*} H^{*} \otimes_{\mathcal{O}_{X}} F, \Phi^{!}(G)\right) \cong \operatorname{RHom}_{\mathcal{A}_{X}}\left(F, f_{\circ}^{*} H \otimes_{\mathcal{O}_{X}} \Phi^{!}(G)\right) .
\end{aligned}
$$

Therefore, by Yoneda lemma we have $\Phi^{!}\left(g_{\circ}^{*} H \otimes \mathcal{O}_{Y} G\right) \cong f_{\circ}^{*} H \otimes \mathcal{O}_{X} \Phi^{!}(G)$, and it is clear that the isomorphism is bifunctorial.

Consider the fiber product of algebraic varieties $X \times_{S} Y$. Let $i: X \times_{S} Y \rightarrow X \times Y$ denote the embedding and let $p: X \times Y \rightarrow X, q: X \times Y \rightarrow Y$ denote the projections, so that $f \circ p \circ i=g \circ q \circ i$.

Lemma 2.34. If $K \in \mathcal{D}_{q c}^{-}\left(X \times_{S} Y, \mathcal{A}_{X}^{\mathrm{opp}} \otimes \mathcal{A}_{Y}\right)$ then the kernel functors $\Phi_{i_{*} K}$ and $\Phi_{i_{*} K}$ are $S$-linear.

Proof: We have

$$
\begin{aligned}
\Phi_{i_{*} K}\left(f_{\circ}^{*} G \otimes_{\mathcal{O}_{X}} F\right) \cong q_{*}\left(p_{\circ}^{*}\left(f_{\circ}^{*} G \otimes_{\mathcal{O}_{X}} F\right) \otimes_{\mathcal{A}_{X}} i_{*} K\right) \cong q_{*}\left(p_{\circ}^{*} f_{\circ}^{*} G \otimes_{\mathcal{O}_{X \times Y}} p_{\circ}^{*} F \otimes_{\mathcal{A}_{X}} i_{*} K\right) \cong \\
\cong q_{*}\left(p_{\circ}^{*} F \otimes_{\mathcal{A}_{X}} i_{*}\left(i_{\circ}^{*} p_{\circ}^{*} f_{\circ}^{*} G \otimes_{\mathcal{O}_{X \times{ }_{S} Y}} K\right)\right) \cong q_{*}\left(p_{\circ}^{*} F \otimes_{\mathcal{A}_{X}} i_{*}\left(i_{\circ}^{*} q_{\circ}^{*} g_{\circ}^{*} G \otimes_{\mathcal{O}_{X \times{ }_{S} Y}} K\right)\right) \cong \\
\cong q_{*}\left(p_{\circ}^{*} F \otimes_{\mathcal{A}_{X}} q_{\circ}^{*} g_{\circ}^{*} G \otimes_{\mathcal{O}_{X \times Y}} i_{*} K\right) \cong g_{\circ}^{*} G \otimes_{\mathcal{O}_{Y}} q_{*}\left(p_{\circ}^{*} F \otimes_{\mathcal{A}_{X}} i_{*} K\right) \cong g_{\circ}^{*} G \otimes_{\mathcal{O}_{Y}} \Phi_{i_{*} K}(F),
\end{aligned}
$$

and similarly for $\Phi_{i_{*} K}$.

A strictly full subcategory $\mathcal{C} \subset \mathcal{D}\left(X, \mathcal{A}_{X}\right)$ is called $S$-linear if for all $F \in \mathcal{C}, G \in \mathcal{D}^{b}\left(S, \mathcal{O}_{S}\right)$ we have $f_{\circ}^{*} G \otimes_{\mathcal{O}_{X}} F \in \mathcal{C}$. For the definition of admissible subcategory see [B, BO1]. 
Lemma 2.35. If $\mathcal{C} \subset \mathcal{D}^{b}\left(X, \mathcal{A}_{X}\right)$ is a strictly full $S$-linear left (resp. right) admissible triangulated subcategory then its left (resp. right) orthogonal is also $S$-linear.

Proof: Assume for example that $\mathcal{C}$ is left admissible and take any $F \in \mathcal{C}, F^{\prime} \in \mathcal{C}^{\perp}$. Then we have

$$
\operatorname{Hom}_{\mathcal{A}_{X}}\left(F, f_{\circ}^{*} G \otimes_{\mathcal{O}_{X}} F^{\prime}\right) \cong \operatorname{Hom}_{\mathcal{A}_{X}}\left(f_{\circ}^{*} G^{*} \otimes_{\mathcal{O}_{X}} F, F^{\prime}\right)=0,
$$

since $f_{\circ}^{*} G^{*} \otimes_{\mathcal{O}_{X}} F \in \mathcal{C}$. Therefore $f_{\circ}^{*} G \otimes_{\mathcal{O}_{X}} F^{\prime} \in \mathcal{C}^{\perp}$, so $\mathcal{C}^{\perp}$ is $S$-linear.

Lemma 2.36. If $\mathcal{D}^{b}\left(X, \mathcal{A}_{X}\right)=\left\langle\mathcal{C}^{\perp}, \mathcal{C}\right\rangle$ is a semiorthogonal decomposition and $\mathcal{C}$ is $S$-linear then we have $f_{*} \operatorname{RHom}_{\mathcal{A}_{X}}\left(F, F^{\prime}\right)=0$ for all $F \in \mathcal{C}, F^{\prime} \in \mathcal{C}^{\perp}$.

Proof: For all $G \in \mathcal{D}^{b}\left(S, \mathcal{O}_{S}\right)$ we have

$$
\operatorname{Hom}_{\mathcal{O}_{S}}\left(G, f_{*} \operatorname{RHom}_{\mathcal{A}_{X}}\left(F, F^{\prime}\right)\right) \cong \operatorname{Hom}_{\mathcal{O}_{X}}\left(f_{\circ}^{*} G, \operatorname{RH}_{\mathcal{H}} m_{\mathcal{A}_{X}}\left(F, F^{\prime}\right)\right) \cong \operatorname{Hom}_{\mathcal{A}_{X}}\left(f_{\circ}^{*} G \otimes_{\mathcal{O}_{X}} F, F^{\prime}\right)=0,
$$

since $f_{\circ}^{*} G \otimes_{\mathcal{O}_{X}} F \in \mathcal{C}$. Therefore $f_{*} \operatorname{RHom}_{\mathcal{A}_{X}}\left(F, F^{\prime}\right)=0$

Proposition 2.37. If $\Phi: \mathcal{D}^{b}\left(X, \mathcal{A}_{X}\right) \rightarrow \mathcal{D}^{b}\left(Y, \mathcal{A}_{Y}\right)$ is $S$-linear, $\Phi^{!}: \mathcal{D}^{b}\left(Y, \mathcal{A}_{Y}\right) \rightarrow \mathcal{D}^{b}\left(X, \mathcal{A}_{X}\right)$ is a right adjoint functor, $f$ and $g$ are projective, and $X$ and $Y$ are smooth then we have a bifunctorial isomorphism

$$
g_{*} \mathrm{RH}_{\mathrm{H} o m_{\mathcal{A}_{Y}}}\left(\Phi(F), F^{\prime}\right) \cong f_{*} \mathrm{RH}^{\circ} m_{\mathcal{A}_{X}}\left(F, \Phi^{!}\left(F^{\prime}\right)\right) .
$$

Proof: Take any $G \in \mathcal{D}^{b}(S)$ and note that

$$
\begin{aligned}
& \operatorname{Hom}_{\mathcal{O}_{S}}\left(G, g_{*} \operatorname{RHom}_{\mathcal{A}_{Y}}\left(\Phi(F), F^{\prime}\right)\right) \cong \operatorname{Hom}_{\mathcal{O}_{Y}}\left(g_{\circ}^{*} G, \operatorname{RHom}_{\mathcal{A}_{Y}}\left(\Phi(F), F^{\prime}\right)\right) \cong \\
& \cong \operatorname{Hom}_{\mathcal{A}_{Y}}\left(g_{\circ}^{*} G \otimes_{\mathcal{O}_{Y}} \Phi(F), F^{\prime}\right) \cong \operatorname{Hom}_{\mathcal{A}_{Y}}\left(\Phi\left(f_{\circ}^{*} G \otimes_{\mathcal{O}_{X}} F\right), F^{\prime}\right) \cong \operatorname{Hom}_{\mathcal{A}_{X}}\left(f_{\circ}^{*} G \otimes_{\mathcal{O}_{X}} F, \Phi^{!} F^{\prime}\right) \cong \\
& \cong \operatorname{Hom}_{\mathcal{O}_{X}}\left(f_{\circ}^{*} G, \mathrm{RH}^{\circ} m_{\mathcal{A}_{X}}\left(F, \Phi^{!} F^{\prime}\right)\right) \cong \operatorname{Hom}_{\mathcal{O}_{X}}\left(G, f_{*} \operatorname{RHom}_{\mathcal{A}_{X}}\left(F, \Phi^{!} F^{\prime}\right)\right) \text {. }
\end{aligned}
$$

On the other hand, $g_{*} \mathrm{R} \mathcal{H} o m_{\mathcal{A}_{Y}}\left(\Phi(F), F^{\prime}\right) \in \mathcal{D}^{b}(S)$ and $f_{*} \operatorname{RH}_{\mathcal{H}} m_{\mathcal{A}_{X}}\left(F, \Phi^{!} F^{\prime}\right) \in \mathcal{D}^{b}(S)$ by assumptions on $X, Y, f$ and $g$. Therefore, by Yoneda lemma we have the desired isomorphism, which is bifunctorial by construction.

2.6. Faithful base changes and kernel functors. Consider morphisms $f:\left(X, \mathcal{A}_{X}\right) \rightarrow\left(S, \mathcal{O}_{S}\right)$ and $g:\left(Y, \mathcal{A}_{Y}\right) \rightarrow\left(S, \mathcal{O}_{S}\right)$ with smooth $S$. For any strict base change $\phi: T \rightarrow S$ we consider the fiber products

$$
X_{T}:=X \times_{S} T, \quad Y_{T}:=Y \times_{S} T, \quad X_{T} \times_{T} Y_{T}=\left(X \times_{S} Y\right) \times_{S} T
$$

and denote the projections $X_{T} \rightarrow X, Y_{T} \rightarrow Y$, and $X_{T} \times_{T} Y_{T} \rightarrow X \times_{S} Y$ also by $\phi$. Further, denote the embeddings $X \times_{S} Y \rightarrow X \times Y$ and $X_{T} \times_{T} Y_{T} \rightarrow X_{T} \times Y_{T}$ by $i$ and $i_{T}$ respectively. For any kernel $K \in \mathcal{D}^{-}\left(X \times Y_{S}, \mathcal{A}_{X}^{\text {opp }} \otimes \mathcal{A}_{Y}\right)$ we denote

$$
K_{T}=\phi^{*} K \in \mathcal{D}^{-}\left(X_{T} \times_{T} Y_{T}, \mathcal{A}_{X}^{\text {opp }} \otimes \mathcal{A}_{Y}\right) .
$$

Definition 2.38. A strict morphism $\phi: T \rightarrow S$ is called faithful for a pair $(X, Y)$ if $\phi$ is faithful with respect to morphisms $f: X \rightarrow S, g: Y \rightarrow S$, and $f \times_{S} g: X \times_{S} Y \rightarrow S$.

Lemma 2.39. Assume that $T \rightarrow S$ is a strict base change faithful for a pair $(X, Y)$. If $T \rightarrow S^{\prime} \rightarrow S$ is a factorization of $T \rightarrow S$ such that $T \rightarrow S^{\prime}$ is strict and finite and $S^{\prime} \rightarrow S$ is strict and smooth, then $S^{\prime} \rightarrow S$ is faithful for the pair $(X, Y)$ and $T \rightarrow S^{\prime}$ is faithful for the pair $\left(X \times_{S} S^{\prime}, Y \times_{S} S^{\prime}\right)$.

Proof: Follows from corollary 2.31 . 
Lemma 2.40. If $\phi: T \rightarrow S$ is a strict base change faithful for a pair $(X, Y)$ then both squares in the commutative diagram

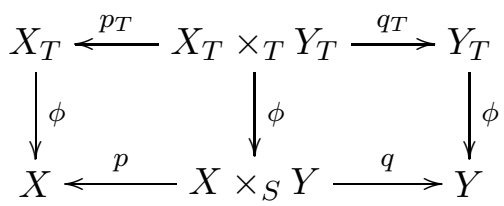

are exact cartesian.

Proof: Apply the "only if" part of lemma 2.25 to the following cartesian diagrams

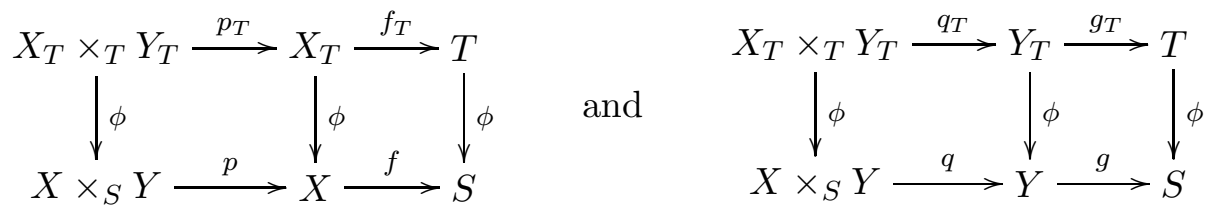

Lemma 2.41. If $\phi: T \rightarrow S$ is a strict base change faithful for a pair $(X, Y)$, and $f$ is projective then we have

$$
\Phi_{i_{T *} K_{T}} \phi^{*}=\phi^{*} \Phi_{i_{*} K}, \quad \Phi_{i_{*} K} \phi_{*}=\phi_{*} \Phi_{i_{T *} K_{T}}, \quad \Phi_{i_{T *} K_{T}} \phi^{*}=\phi^{*} \Phi_{i_{*} K}^{!}, \quad \text { and } \quad \Phi_{i_{*} K}^{!_{*}} \phi_{*}=\phi_{*} \Phi_{i_{T *} K_{T}} .
$$

Proof: Put for short $\Phi=\Phi_{i_{*} K}, \Phi^{!}=\Phi_{i_{*} K}^{!}, \Phi_{T}=\Phi_{i_{T *} K_{T}}, \Phi_{T}^{!}=\Phi_{i_{T *} K_{T}}$. Note that $\phi$ is strict, hence $\phi^{*}=\phi_{0}^{*}$. For any $F \in \mathcal{D}^{-}(X), G \in \mathcal{D}^{-}\left(X_{T}\right)$ we have

$$
\begin{gathered}
\Phi_{T} \phi^{*}(F)=q_{T *}\left(p_{T \circ}{ }^{*} \phi^{*} F \otimes_{\mathcal{A}_{X}} K_{T}\right) \cong q_{T *}\left(\phi^{*} p_{\circ}^{*} F \otimes_{\mathcal{A}_{X}} \phi^{*} K\right) \cong \\
\\
\cong q_{T *} \phi^{*}\left(p_{\circ}^{*} F \otimes_{\mathcal{A}_{X}} K\right) \cong \phi^{*} q_{*}\left(p_{\circ}^{*} F \otimes_{\mathcal{A}_{X}} K\right)=\phi^{*} \Phi(F), \\
\begin{array}{r}
\Phi \phi_{*}(G)=q_{*}\left(p_{\circ}{ }^{*} \phi_{*} G \otimes_{\mathcal{A}_{X}} K\right) \cong q_{*}\left(\phi_{*} p_{T \circ}{ }^{*} G \otimes_{\mathcal{A}_{X}} K\right) \cong \\
\cong q_{*} \phi_{*}\left(p_{T \circ}{ }^{*} G \otimes_{\mathcal{A}_{X}} \phi^{*} K\right) \cong \phi_{*} q_{T *}\left(p_{T \circ}{ }^{*} G \otimes_{\mathcal{A}_{X}} K_{T}\right)=\phi_{*} \Phi_{T}(G) .
\end{array}
\end{gathered}
$$

Note that $\phi$ has finite Tor-dimension since $S$ is smooth, and $q$ is projective because $f$ is. Hence for any $F \in \mathcal{D}^{+}(X), G \in \mathcal{D}^{+}\left(X_{T}\right)$ using lemma 2.32 we deduce

$$
\begin{aligned}
\Phi_{T}^{!} \phi^{*}(F)=p_{T *} \operatorname{RHom}_{\mathcal{A}_{Y}}\left(K_{T}, q_{T \circ} ! \phi^{*} F\right) & \cong p_{T *} \operatorname{RH} \operatorname{com}_{\mathcal{A}_{Y}}\left(\phi^{*} K, \phi^{*} q_{\circ}^{!} F\right) \cong \\
& \cong p_{T *} \phi^{*} \operatorname{RHom}_{\mathcal{A}_{Y}}\left(K, q_{\circ}^{!} F\right) \cong \phi^{*} p_{*} \operatorname{RHom}_{\mathcal{A}_{Y}}\left(K, q_{\circ}^{!} F\right)=\phi^{*} \Phi^{!}(F),
\end{aligned}
$$

and using proposition 2.24 we deduce

$$
\begin{aligned}
& \Phi^{!} \phi_{*}(G)=p_{*} \mathrm{RH}^{\circ} m_{\mathcal{A}_{Y}}\left(K, q_{\circ}{ }^{!} \phi_{*} F\right) \cong p_{*} \mathrm{RH}^{\circ} m_{\mathcal{A}_{Y}}\left(K, \phi_{*} q_{T \circ}{ }^{!} F\right) \cong \\
& \cong p_{*} \phi_{*} \mathrm{RH}^{\circ o m_{\mathcal{A}_{Y}}}\left(\phi^{*} K, q_{T \circ}{ }^{!} F\right) \cong \phi_{*} p_{T *} \mathrm{RH} \mathcal{H}_{\mathcal{A}_{Y}}\left(K_{T}, q_{T \circ}^{!} F\right)=\phi_{*} \Phi_{T}^{!}(F) \text {. }
\end{aligned}
$$

Lemma 2.42. Assume that $\phi$ is faithful for the pair $(X, Y)$ and $K \in \mathcal{D}^{b}\left(X \times_{S} Y\right)$.

(a) If $K$ has finite Tor-amplitude over $Y$ then $K_{T}$ has finite Tor-amplitude over $Y_{T}$.

(b) If $K$ has finite Ext-amplitude over $X$ then $K_{T}$ has finite Ext-amplitude over $X_{T}$.

(c) If supp $K$ is projective over $X$ then If supp $K_{T}$ is projective over $X_{T}$.

(d) If supp $K$ is projective over $Y$ then If supp $K_{T}$ is projective over $Y_{T}$. 
Proof: $(a)$ and $(b)$ follow from corollary D.46; $(c)$ and $(d)$ are evident.

Let $X_{1}, \ldots, X_{n}$, and $Y$ be smooth Azumaya varieties projective over the same smooth algebraic variety $S$. Consider kernels $K_{1} \in \mathcal{D}^{-}\left(X_{1} \times_{S} Y, \mathcal{A}_{X_{1}}^{\text {opp }} \otimes \mathcal{A}_{Y}\right), \ldots, K_{n} \in \mathcal{D}^{-}\left(X_{n} \times_{S} Y, \mathcal{A}_{X_{n}}^{\text {opp }} \otimes \mathcal{A}_{Y}\right)$ and denote by $\Phi_{1}: \mathcal{D}^{b}\left(X_{1}\right) \rightarrow \mathcal{D}^{b}(Y), \ldots, \Phi_{n}: \mathcal{D}^{b}\left(X_{n}\right) \rightarrow \mathcal{D}^{b}(Y)$ the corresponding kernel functors. Let $\phi: T \rightarrow S$ be a strict base change. We use the notations $X_{k T}, Y_{T}, f_{k T}, g_{T}, \Phi_{k T}, \Phi_{k T}^{!}$in an evident sense. Finally, assume that $K_{i}$ have finite Tor-amplitude over $Y$, finite Ext-amplitude over $X_{i}$, and that morphisms $g$ and $f_{i}$ are projective for all $i$. Then the above lemma together with lemma 2.4 imply that all functors preserve bounded derived categories and that $\Phi_{i}^{!}, \Phi_{i T}^{!}$are right adjoint to $\Phi_{i}, \Phi_{i T}$.

Proposition 2.43. Assume that $\phi$ is faithful for the pairs $\left(X_{1}, Y\right),\left(X_{2}, Y\right)$.

(i) If the subcategory $\Phi_{2}\left(\mathcal{D}^{b}\left(X_{2}\right)\right)$ is right orthogonal to $\Phi_{1}\left(\mathcal{D}^{b}\left(X_{1}\right)\right)$ then the subcategory $\Phi_{2 T}\left(\mathcal{D}^{b}\left(X_{2 T}\right)\right)$ is right orthogonal to $\Phi_{1 T}\left(\mathcal{D}^{b}\left(X_{1 T}\right)\right)$.

(ii) If $\Phi_{1}$ is fully faithful then $\Phi_{1 T}$ is fully faithful.

(iii) If $\Phi_{1}^{!}$is fully faithful then $\Phi_{1 T}^{!}$is fully faithful.

(iv) If $\Phi_{1}$ is an equivalence then $\Phi_{1 T}$ is an equivalence.

Proof: First we note that $\Phi_{2}\left(\mathcal{D}^{b}\left(X_{2}\right)\right)$ is right orthogonal to $\Phi_{1}\left(\mathcal{D}^{b}\left(X_{1}\right)\right)$ iff $\Phi_{1}^{!} \Phi_{2}=0$ and $\Phi_{2 T}\left(\mathcal{D}^{b}\left(X_{2 T}\right)\right)$ is right orthogonal to $\Phi_{1 T}\left(\mathcal{D}\left(X_{1 T}\right)\right)$ iff $\Phi_{1 T}^{!} \Phi_{2 T}=0$. So, in $(i)$ we must check that $\Phi_{1}^{!} \Phi_{2}=0$ implies $\Phi_{1 T}^{!} \Phi_{2 T}=0$.

Similarly, $\Phi_{1}$ is fully faithful iff the adjunction morphism $\operatorname{id}_{\mathcal{D}^{b}\left(X_{1}\right)} \rightarrow \Phi_{1}^{!} \Phi_{1}$ is an isomorphism and $\Phi_{1 T}$ is fully faithful iff the adjunction morphism $\mathrm{id}_{\mathcal{D}^{b}\left(X_{1 T}\right)} \rightarrow \Phi_{1 T}^{!} \Phi_{1 T}$ is an isomorphism. So, in $(i i)$ we must check that $\mathrm{id}_{\mathcal{D}^{b}\left(X_{1}\right)} \cong \Phi_{1}^{!} \Phi_{1}$ implies $\operatorname{id}_{\mathcal{D}^{b}\left(X_{1 T}\right)} \cong \Phi_{1 T}^{!} \Phi_{1 T}$.

By lemma 2.39 it suffices to consider the case when $\phi$ is smooth and the case when $\phi$ is finite.

If $\phi$ is smooth then $X_{1 T}, X_{2 T}$, and $Y_{T}$ are smooth varieties, hence $\Phi_{1}^{!}$and $\Phi_{1 T}^{!}$are usual kernel functors by lemma 2.2. Moreover, if $\mathcal{F}_{1}$ is a perfect spanning class for $X_{1}, \mathcal{F}_{2}$ is a perfect spanning class for $X_{2}$, and $\mathcal{G}$ is a perfect spanning class for $T$ then $f_{2 T \circ}^{*} \mathcal{G} \otimes_{\mathcal{O}_{X_{2}}} \mathcal{F}_{2}$ is a perfect spanning class for $X_{2 T}$ by lemma 2.12. Further, for all $F \in \mathcal{F}_{2}, G \in \mathcal{G}$ by lemma 2.41 and lemma 2.34 we have

$$
\begin{aligned}
& \Phi_{1 T}^{!} \circ \Phi_{2 T}\left(f_{2 T \circ}^{*} G \otimes \mathcal{O}_{X_{2 T}} F\right) \cong \Phi_{1 T}^{!}\left(g_{T \circ}^{*} G \otimes_{\mathcal{O}_{Y_{T}}} \Phi_{2 T}\left(\phi^{*} F\right)\right) \cong \\
& \cong f_{1 T \circ}^{*} G \otimes \mathcal{O}_{X_{1 T}} \Phi_{1 T}^{!}\left(\phi^{*} \Phi_{2}(F)\right) \cong f_{1 T \circ}^{*} G \otimes_{\mathcal{O}_{X_{1 T}}} \phi^{*} \Phi_{1}^{!}\left(\Phi_{2}(F)\right) .
\end{aligned}
$$

We deduce that $\Phi_{1}^{!} \Phi_{2}=0$ implies that $\Phi_{1 T}^{!} \Phi_{2 T}$ vanishes on $f_{2 T \circ}^{*} \mathcal{G} \otimes \mathcal{O}_{X_{2 T}} \mathcal{F}_{2}$, hence $\Phi_{1 T}^{!} \Phi_{2 T}=0$ by proposition 2.14 .

Similarly, $f_{1 T \circ}^{*} \mathcal{G} \otimes_{\mathcal{O}_{X_{1 T}}} \mathcal{F}_{1}$ is a perfect spanning class for $X_{1 T}$ and for all $F \in \mathcal{F}_{1}, G \in \mathcal{G}$ by lemma 2.41 and lemma 2.34 we have

$$
\begin{aligned}
\Phi_{1 T}^{!} \circ \Phi_{1 T}\left(f_{1 T \circ}^{*} G \otimes \mathcal{O}_{X_{1 T}} \phi^{*} F\right) \cong \Phi_{1 T}^{!}\left(g_{T \circ}^{*} G \otimes_{\mathcal{O}_{Y_{T}}} \Phi_{1 T}\left(\phi^{*} F\right)\right) \cong \\
\\
\quad \cong f_{1 T \circ}^{*} G \otimes_{\mathcal{O}_{X_{1 T}}} \Phi_{1 T}^{!}\left(\phi^{*} \Phi_{1}(F)\right) \cong f_{1 T \circ}^{*} G \otimes_{\mathcal{O}_{X_{1 T}}} \phi^{*} \Phi_{1}^{!}\left(\Phi_{1}(F)\right) .
\end{aligned}
$$

We deduce that $\mathrm{id}_{\mathcal{D}^{b}\left(X_{1}\right)} \cong \Phi_{1}^{!} \Phi_{1}$ implies that $\Phi_{1 T}^{!} \Phi_{1 T}$ is isomorphic to $\mathrm{id}_{\mathcal{D}^{b}\left(X_{1 T}\right)}$ on $f_{1 T \circ}^{*} \mathcal{G} \otimes_{\mathcal{O}_{X_{1 T}}} \mathcal{F}_{1}$, hence $\operatorname{id}_{\mathcal{D}^{b}\left(X_{1 T}\right)} \cong \Phi_{1 T}^{!} \Phi_{1 T}$ by corollary 2.15 .

If $\phi$ is finite then lemma 2.41 implies that

$$
\phi_{*} \Phi_{1 T}^{!} \Phi_{2 T} \cong \Phi_{1}^{!} \phi_{*} \Phi_{2 T} \cong \Phi_{1}^{!} \Phi_{2} \phi_{*}, \quad \text { and } \quad \phi_{*} \Phi_{1 T}^{!} \Phi_{1 T} \cong \Phi_{1}^{!} \phi_{*} \Phi_{1 T} \cong \Phi_{1}^{!} \Phi_{1} \phi_{*} .
$$

We deduce that $\Phi_{1}^{!} \Phi_{2}=0$ implies $\phi_{*} \Phi_{1 T}^{!} \Phi_{2 T}=0$, hence $\Phi_{1 T}^{!} \Phi_{2 T}=0$ by lemma 2.8 a), and that $\mathrm{id}_{\mathcal{D}^{b}\left(X_{1}\right)} \cong \Phi_{1}^{!} \Phi_{1}$ implies $\phi_{*} \cong \phi_{*} \Phi_{1 T}^{!} \Phi_{1 T}$, hence $\operatorname{id}_{\mathcal{D}^{b}\left(X_{1 T}\right)} \cong \Phi_{1 T}^{!} \Phi_{1 T}$ by lemma $\left.2.8 \mathrm{~b}\right)$.

The claim (iii) can be proved by the similar arguments as (ii) using the fact that $\Phi_{1}^{!}$is fully faithful iff $\Phi_{1} \Phi_{1}^{!} \cong \mathrm{id}_{\mathcal{D}^{b}(Y)}$ and $\Phi_{1 T}^{!}$is fully faithful iff $\Phi_{1 T} \Phi_{1 T}^{!} \cong \mathrm{id}_{\mathcal{D}^{b}\left(Y_{T}\right)}$. Finally, $(i v)$ follows from (ii) combined with $(i i i)$. 
Corollary 2.44. In the conditions of the above proposition if $g_{*} \mathrm{RHom} \mathcal{A}_{Y}\left(E, \Phi_{2}\left(\mathcal{D}^{b}\left(X_{2}\right)\right)\right)=0$ for some $E \in \mathcal{D}^{b}(Y)$ then $g_{T *} \mathrm{RH}_{\text {or }}{ }_{\mathcal{A}_{Y_{T}}}\left(\phi^{*} E, \Phi_{2 T}\left(\mathcal{D}^{b}\left(X_{2 T}\right)\right)\right)=0$.

Proof: We take $X_{1}=S$, consider $E$ as a kernel on $X_{1} \times_{S} Y=S \times_{S} Y \cong Y$, put $\Phi_{1}=\Phi_{E}$, and apply proposition 2.43 (i) and lemma 2.36.

Theorem 2.45. In the conditions of the proposition if

$$
\mathcal{D}^{b}(Y)=\left\langle\Phi_{1}\left(\mathcal{D}^{b}\left(X_{1}\right)\right), \ldots, \Phi_{n}\left(\mathcal{D}^{b}\left(X_{n}\right)\right)\right\rangle
$$

is a semiorthogonal decomposition for $Y$ then

$$
\mathcal{D}^{b}\left(Y_{T}\right)=\left\langle\Phi_{1 T}\left(\mathcal{D}^{b}\left(X_{1 T}\right)\right), \ldots, \Phi_{n T}\left(\mathcal{D}^{b}\left(X_{n T}\right)\right)\right\rangle
$$

is a semiorthogonal decomposition for $Y_{T}$.

Proof: By proposition 2.43 it suffices to check that the orthogonal to the subcategory $\mathcal{D}^{\prime} \subset \mathcal{D}^{b}\left(Y_{T}\right)$ generated by $\Phi_{1 T}\left(\mathcal{D}^{b}\left(X_{1 T}\right)\right), \ldots, \Phi_{n T}\left(\mathcal{D}^{b}\left(X_{n T}\right)\right)$ is zero. Choose a perfect spanning class $\mathcal{F}$ for $Y$ and a perfect spanning class $\mathcal{G}$ for $T$. Then any object $F \in \mathcal{F}$ can be decomposed with respect to the initial semiorthogonal decomposition. Applying lemma 2.41 and lemma 2.34 we deduce that $g_{T 0}^{*} G \otimes_{\mathcal{O}_{Y_{T}}} \phi^{*}(F)$ is contained in $\mathcal{D}^{\prime}$ for all $F \in \mathcal{F}, G \in \mathcal{G}$. Hence $\mathcal{D}^{\prime}$ contains a perfect spanning class for $Y_{T}$, hence $\mathcal{D}^{\prime \perp}=0$ by lemma 2.13 .

2.7. Relative Serre functor and Bridgeland's trick. Assume that $X$ and $Y$ are Azumaya varieties, $X$ is smooth, and $f: X \rightarrow S, g: Y \rightarrow S$ are projective morphisms into a smooth algebraic variety $S$. If $\Phi: \mathcal{D}^{b}(X) \rightarrow \mathcal{D}^{b}(Y)$ is an $S$-linear functor then for all $F, F^{\prime} \in \mathcal{D}^{b}(X), G \in \mathcal{D}^{b}(S)$ we have a diagram

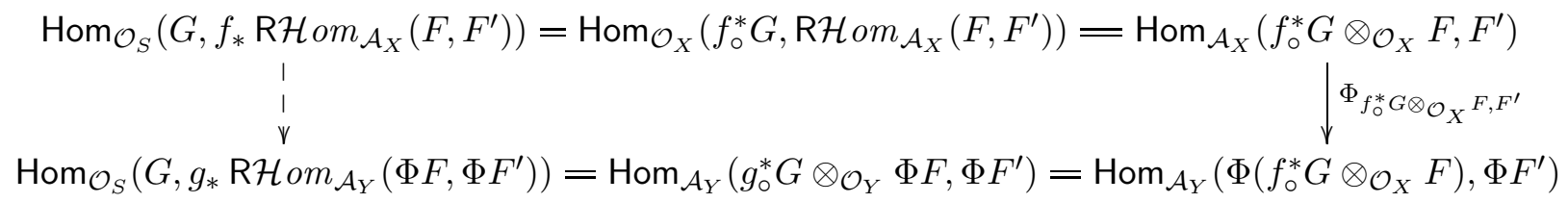

where the dashed arrow is the unique morphism making the diagram commutative. Note that we have $f_{*} \operatorname{RHom}_{\mathcal{A}_{X}}\left(F, F^{\prime}\right) \in \mathcal{D}^{b}(S)$, since $X$ is smooth and $f$ is projective and the dashed arrow is evidently functorial in $G$. Hence by the Yoneda lemma it is induced by a unique morphism

$$
\Phi_{F, F^{\prime}}^{S}: f_{*} \mathrm{RH}_{\mathrm{H}} m_{\mathcal{A}_{X}}\left(F, F^{\prime}\right) \rightarrow g_{*} \mathrm{R} \mathcal{H} o m_{\mathcal{A}_{Y}}\left(\Phi F, \Phi F^{\prime}\right) .
$$

Lemma 2.46. Morphisms $\Phi_{F, F^{\prime}}^{S}$ are bifunctorial in $F, F^{\prime}$.

Proof: Use uniqueness of $\Phi_{F, F^{\prime}}^{S}$ and functorial properties of the above commutative diagram.

Definition 2.47. (cf. [BK, BO3]) A covariant additive functor $\mathrm{S}_{X / S}: \mathcal{D}^{b}(X) \rightarrow \mathcal{D}^{b}(X)$ is called a relative Serre functor, if it is an $S$-linear category equivalence and for all objects $F, F^{\prime} \in \mathcal{D}^{b}(X)$ there are given bifunctorial isomorphisms

$$
\varphi_{F, F^{\prime}}: f_{*} \operatorname{RH}^{\circ o m_{\mathcal{A}_{X}}}\left(F, F^{\prime}\right) \rightarrow\left(f_{*} \mathrm{RH}_{\mathcal{H}} m_{\mathcal{A}_{X}}\left(F^{\prime}, \mathrm{S}_{X / S} F\right)\right)^{*} .
$$

Let $\omega_{X / S}$ denote the relative canonical class of the underlying algebraic variety of $X$ over $S$.

Lemma 2.48. ( cf. $[\mathrm{BK}])$ The functor $\mathrm{S}_{X / S}(F)=\omega_{X / S}[\operatorname{dim} X-\operatorname{dim} S] \otimes_{\mathcal{O}_{X}} F$ is a relative Serre functor, and any relative Serre functor is canonically isomorphic to it. 
Proof: Using the duality theorem we get

$$
\begin{aligned}
& \left(f_{*} \mathrm{R} \mathcal{H} o m_{\mathcal{A}_{X}}\left(F, F^{\prime}\right)\right)^{*} \cong \mathrm{R} \mathcal{H} o m_{\mathcal{O}_{S}}\left(f_{*} \mathrm{RH} \mathcal{H}_{\mathcal{A}_{X}}\left(F, F^{\prime}\right), \mathcal{O}_{S}\right) \cong \\
& \cong f_{*} \mathrm{RH} \operatorname{Hom}_{\mathcal{O}_{X}}\left(\mathrm{RH} \mathcal{H}_{\mathcal{A}_{X}}\left(F, F^{\prime}\right), f_{\circ}^{!} \mathcal{O}_{S}\right) \cong f_{*} \mathrm{RH} o m_{\mathcal{A}_{X}}\left(F^{\prime}, f_{\circ}^{!} \mathcal{O}_{S} \otimes_{\mathcal{O}_{X}} F\right) \text {. }
\end{aligned}
$$

But $f_{0}^{!} \mathcal{O}_{S} \cong \omega_{X / S}[\operatorname{dim} X-\operatorname{dim} S]$, and it is clear that $\mathrm{S}_{X / S}(F)=\omega_{X / S}[\operatorname{dim} X-\operatorname{dim} S] \otimes_{\mathcal{O}_{X}} F$ is an $S$-linear category equivalence. Dualizing the above isomorphism we deduce that it is a relative Serre functor. The second part is proved in the same way as the uniqueness of a usual Serre functor.

Corollary 2.49. If $X$ is smooth, $\Phi: \mathcal{D}^{b}(Y) \rightarrow \mathcal{D}^{b}(X)$ is an $S$-linear equivalence and $\omega_{X / S} \cong f_{\circ}^{*} L$ for a line bundle $L$ on $S$, then $Y$ is smooth, $\operatorname{dim} Y=\operatorname{dim} X$, and $\omega_{Y / S} \cong g_{\circ}^{*} L$.

Proof: If $X$ is smooth then $\mathcal{D}^{b}(X)$ is Ext-bounded, hence $\mathcal{D}^{b}(Y)$ is Ext-bounded, hence $Y$ is smooth (see lemma D.22). Further, it is clear that $S_{Y / S}=\Phi^{!} \circ S_{X / S} \circ \Phi$ is a relative Serre functor on $Y$. But denoting $d=\operatorname{dim} X-\operatorname{dim} S$ we get

$$
\left(\Phi^{!} \circ S_{X / S} \circ \Phi\right)(F)=\Phi^{!}\left(f_{\circ}^{*} L[d] \otimes_{\mathcal{O}_{X}} \Phi(F)\right) \cong g_{\circ}^{*} L[d] \otimes_{\mathcal{O}_{Y}} \Phi^{!}(\Phi(F)) \cong g_{\circ}^{*} L[d] \otimes_{\mathcal{O}_{Y}} F,
$$

and it remains to apply the uniqueness of the relative Serre functor.

Proposition 2.50. If $X$ is smooth and connected, $\omega_{X} \cong f_{0}^{*} L$ for a line bundle $L$ on $S$, and $Y$ is not empty, then any fully faithful $S$-linear functor $\Phi: \mathcal{D}^{b}(Y) \rightarrow \mathcal{D}^{b}(X)$ is an equivalence. Furthermore, in this case $Y$ is smooth, connected, $\operatorname{dim} Y=\operatorname{dim} X$ and $\omega_{Y} \cong g_{\circ}^{*} L$.

Proof: The functor $\Phi$ is an equivalence of $\mathcal{D}^{b}(Y)$ with a strictly full right admissible $S$-linear triangulated subcategory $\mathcal{C} \subset \mathcal{D}^{b}(X)$. Consider a semiorthogonal decomposition $\left(\mathcal{C}^{\perp}, \mathcal{C}\right)$ for $\mathcal{D}^{b}(X)$. Note that the Serre functor $\mathrm{S}_{X / S}(F)=f_{\circ}^{*} L \otimes_{\mathcal{O}_{X}} F[\operatorname{dim} X-\operatorname{dim} S]$ preserves any $S$-linear subcategory of $\mathcal{D}^{b}(X)$. Therefore for all $F \in \mathcal{C}, F^{\prime} \in \mathcal{C}^{\perp}$ we have $f_{*} \mathrm{RH} \mathcal{H o m}_{\mathcal{A}_{X}}\left(F^{\prime}, F\right) \cong\left(f_{*} \mathrm{RHom} \mathcal{A}_{X}\left(F, \mathrm{~S}_{X / S} F^{\prime}\right)\right)^{*}=0$, which implies $\operatorname{Hom}_{\mathcal{A}_{X}}\left(F^{\prime}, F\right) \cong \operatorname{Hom}_{\mathcal{O}_{S}}\left(\mathcal{O}_{S}, f_{*} \operatorname{RH}^{\prime} \operatorname{mom}_{\mathcal{A}_{X}}\left(F^{\prime}, F\right)\right)=0$. Thus the subcategories $\mathcal{C}$ and $\mathcal{C}^{\perp}$ are totally orthogonal. But the category $\mathcal{D}^{b}(X)$ is indecomposable by [Br1], hence $\mathcal{C}^{\perp}=0$ and $\Phi$ is an equivalence. The rest of the claim follows from corollary 2.49 .

\section{The Universal hyperplane SECTION}

Though $Y$ is in general an Azumaya variety, for unburdening of the notation we drop all the Azumaya stuff in this section. However, a patient reader can easily fill in the details and check that section 2 and appendix D contain a verification of all necessary facts concerning Azumaya varieties.

The goal of this section is to make the first step in the proof of the theorem 1.2 as indicated in the introduction. So assume that we have data (D.1)-(D.5) satisfying the conditions (C.1)-(C.6). In fact, the condition (C.8) is never used in this section, while the condition (C.7) will be explicitly stated in theorem 3.1.

3.1. Notation. Let $\mathbf{P}=\mathbb{P}\left(V^{*}\right) \backslash \mathbf{Z}$ and denote by $\mathcal{L}$ the restriction to $\mathbf{P}$ of $\mathcal{O}_{\mathbb{P}\left(V^{*}\right)}(-1)$. Recall that (C.6) implies that we have the following exact sequence on $X \times Y$

$$
0 \rightarrow E_{1} \otimes F_{1} \stackrel{e}{\longrightarrow} E_{2} \otimes F_{2} \rightarrow i_{*} \mathcal{E} \rightarrow 0,
$$

where $\mathcal{E}$ is a coherent sheaf on $Q(X, Y)$ and $i: Q(X, Y) \rightarrow X \times Y$ denotes the embedding.

Consider $\mathcal{X}=X \times \mathbf{P}$, and let $\mathcal{X}_{1} \subset X \times \mathbf{P}$ be the universal hyperplane section of $X$, i.e. the zero locus of the canonical section of the line bundle $\mathcal{O}_{X}(1) \otimes \mathcal{L}^{*}$. Let $\alpha: \mathcal{X}_{1} \rightarrow X \times \mathbf{P}$ denote the embedding. Then we have a resolution

$$
0 \rightarrow \mathcal{O}_{X}(-1) \otimes \mathcal{L} \rightarrow \mathcal{O}_{X \times \mathbf{P}} \rightarrow \alpha_{*} \mathcal{O}_{\mathcal{X}_{1}} \rightarrow 0
$$


Denote by $\beta$ the embedding of $Y$ to $\mathbf{P} \times Y$ given by the graph of $g$. We will show below that the composition

$$
Q(X, Y) \stackrel{i}{\longrightarrow} X \times Y \stackrel{\mathrm{id}_{X} \times \beta}{\longrightarrow} X \times \mathbf{P} \times Y
$$

factors through $\mathcal{X}_{1} \times Y$. Let $j: Q(X, Y) \rightarrow \mathcal{X}_{1} \times Y$ denote the corresponding closed embedding, so that $\left(\operatorname{id}_{X} \times \beta\right) \circ i=\left(\alpha \times \operatorname{id}_{Y}\right) \circ j$ and we have a commutative square

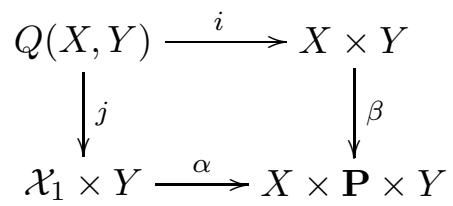

where we write $\alpha$ instead of $\alpha \times \mathrm{id}_{Y}$ and $\beta$ instead of $\operatorname{id}_{X} \times \beta$ for brevity. Further, consider an object $\mathcal{E}^{*}=\mathrm{RHom}_{Q(X, Y)}\left(\mathcal{E}, \mathcal{O}_{Q(X, Y)}\right) \in \mathcal{D}(Q(X, Y))$ and denote

$$
\mathcal{E}_{1}=j_{*} \mathcal{E}, \quad \mathcal{E}^{\# t}=\mathcal{E}^{*}(1+t-i, 1), \quad \mathcal{E}_{1}^{\# t}=j_{*} \mathcal{E}^{\# t} \otimes \mathcal{L}^{* \otimes t}[\operatorname{dim} X-1],
$$

where $\mathcal{O}(a, b)$ stands for $\mathcal{O}_{X}(a) \otimes \mathcal{O}_{Y}(b)$. Let

$$
B:=\operatorname{dim} X+\operatorname{dim} \mathbf{P}-\operatorname{codim}_{Y \times Y}\left(Y \times_{\mathbf{P}} Y\right) .
$$

Note that the condition (C.7) is equivalent to $B=2 i-1$.

The main result of this section is the following

Theorem 3.1. We have $B \geq 2 i-1$. If $B=2 i-1$ then the functor $\Phi_{\mathcal{E}_{1}}: \mathcal{D}^{b}(Y) \rightarrow \mathcal{D}^{b}\left(\mathcal{X}_{1}\right)$ is fully faithful. Moreover, in this case $Y$ is smooth.

The proof goes as follows. First of all we note that the functor $\Phi_{\mathcal{E}_{1}^{\# 0}}$ is left adjoint to $\Phi_{\mathcal{E}_{1}}$, so the claim of the theorem reduces to the computation of the convolution $\mathcal{E}_{1} \circ \mathcal{E}_{1}^{\# 0}$. However, a straightforward computation is not as easy. Roughly speaking, we have to compute RHom between two sheaves on a singular divisor, when we only know resolutions of these sheaves on the ambient variety. So we use another argument to circumvent this. First of all we consider the convolutions $\mathcal{E}_{1} \circ \mathcal{E}_{1}^{\# t}$ for all $t \in \mathbb{Z}$. Second, we consider objects $\tilde{\mathcal{E}}=i^{*} i_{*} \mathcal{E} \in \mathcal{D}(Q(X, Y)), \tilde{\mathcal{E}}_{1}=j_{*} \tilde{\mathcal{E}} \in \mathcal{D}\left(\mathcal{X}_{1} \times Y\right)$ and the convolutions $\tilde{\mathcal{E}}_{1} \circ \mathcal{E}_{1}^{\# t}$. Since $i$ is a divisorial embedding $(Q(X, Y)$ is a divisor of bidegree $(1,1))$ we have an exact triangle $i^{*} i_{*} \mathcal{E} \rightarrow \mathcal{E} \rightarrow$

$\mathcal{E}(-1,-1)[2]$ which gives an exact triangle of convolutions $\tilde{\mathcal{E}}_{1} \circ \mathcal{E}_{1}^{\# t} \rightarrow \mathcal{E}_{1} \circ \mathcal{E}_{1}^{\# t} \rightarrow \mathcal{E}_{1} \circ \mathcal{E}_{1}^{\#(t-1)}[2]$. It turns out that the first term can be easily computed for $0 \leq t \leq i$. Comparing this result with uniform (in $t$ ) cohomological boundedness of $\mathcal{E}_{1} \circ \mathcal{E}_{1}^{\# t}$ we deduce the theorem.

Now we give a detailed proof.

\subsection{Preparations.}

Lemma 3.2. The universal hyperplane section $\mathcal{X}_{1}$ is smooth, projective over $\mathbf{P}$ and its relative dimension over $\mathbf{P}$ equals $\operatorname{dim} X-1$.

Proof: It is easy to see that the projection $\mathcal{X}_{1} \rightarrow X$ is smooth (in fact it is a projectivization of a vector bundle), hence $\mathcal{X}_{1}$ is smooth. On the other hand, the fibers of $\mathcal{X}_{1}$ over $\mathbf{P}$ are hyperplane sections of $X$, hence all of them have dimension $\operatorname{dim} X-1$ by (C.1) and projective since $X$ is.

Lemma 3.3. We have $\mathcal{X}_{1} \times_{\mathbf{P}} Y \cong Q(X, Y)$. If $j$ denotes the induced embedding $Q(X, Y) \rightarrow \mathcal{X}_{1} \times Y$ then $\left(\mathrm{id}_{X} \times \beta\right) \circ j=\left(\alpha \times \mathrm{id}_{Y}\right) \circ i$ and the square (4) is commutative. 
Proof: It is clear that $\mathcal{X}_{1} \times_{\mathbf{P}} Y$ coincides with the zero locus of the canonical section of the line bundle $\mathcal{O}_{X}(1) \otimes \mathcal{L}^{*}$ on $\mathcal{X} \times_{\mathbf{P}} Y=(X \times \mathbf{P}) \times_{\mathbf{P}} Y=X \times Y$. On the other hand, on $\mathcal{X} \times_{\mathbf{P}} Y$ we have $\mathcal{L}^{*} \cong \mathcal{O}_{Y}(1)$ hence $\mathcal{X}_{1} \times_{\mathbf{P}} Y$ is the zero locus of the canonical section of the line bundle $\mathcal{O}_{X}(1) \otimes \mathcal{O}_{Y}(1)$, hence coincides with $Q(X, Y)$. It remains to note that the canonical embedding $(X \times \mathbf{P}) \times_{\mathbf{P}} Y \rightarrow X \times \mathbf{P} \times Y$ coincides

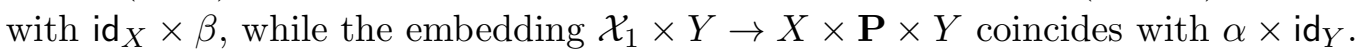

Lemma 3.4. An object $\mathcal{E}^{*}=\mathrm{RH} \operatorname{Hom}_{Q(X, Y)}\left(\mathcal{E}, \mathcal{O}_{Q(X, Y)}\right) \in \mathcal{D}(Q(X, Y))$ is a coherent sheaf and we have the following exact sequence on $X \times Y$

$$
0 \rightarrow E_{2}^{*}(-1) \otimes F_{2}^{*}(-1) \stackrel{e^{*}}{\longrightarrow} E_{1}^{*}(-1) \otimes F_{1}^{*}(-1) \rightarrow i_{*} \mathcal{E}^{*} \rightarrow 0 .
$$

Proof: Applying the functor $\mathrm{RHom}\left(-, \mathcal{O}_{X \times Y}(-1,-1)\right)$ to $(2)$ we obtain a triangle

$$
E_{2}^{*}(-1) \otimes F_{2}^{*}(-1) \stackrel{e^{*}}{\longrightarrow} E_{1}^{*}(-1) \otimes F_{1}^{*}(-1) \rightarrow \operatorname{RHom}\left(i_{*} \mathcal{E}, \mathcal{O}_{X \times Y}(-1,-1)\right)[1]
$$

on $X \times Y$. Since $e$ is an isomorphism at the generic point of $X \times Y$, we deduce that $e^{*}$ is an embedding, hence the third term of the triangle is a sheaf. On the other hand, $i$ is a divisorial embedding, hence $\mathcal{O}_{Q(X, Y)}=i^{*} \mathcal{O}_{X \times Y}=i^{!} \mathcal{O}_{X \times Y}(-1,-1)[1]$, and by the duality theorem we have

$$
i_{*} \operatorname{RHom}\left(\mathcal{E}, \mathcal{O}_{Q(X, Y)}\right)=i_{*} \operatorname{RHom}\left(\mathcal{E}, i^{!} \mathcal{O}_{X \times Y}(-1,-1)\right)[1]=\operatorname{RH} o m\left(i_{*} \mathcal{E}, \mathcal{O}_{X \times Y}(-1,-1)\right)[1] .
$$

Since $i_{*}$ is exact this implies that $\mathcal{E}^{*}=\operatorname{RHom}\left(\mathcal{E}, \mathcal{O}_{Q(X, Y)}\right)$ is a coherent sheaf on $Q(X, Y)$ and $(7)$ is exact.

Lemma 3.5. The sheaf $\mathcal{E}_{1}$ is a perfect complex on $\mathcal{X}_{1} \times Y$.

Proof: Note that the sheaf $\mathcal{E}_{1}=j_{*} \mathcal{E}$ on $\mathcal{X}_{1} \times Y$ has finite Tor-amplitude over $Y$. Indeed, consider the commutative square (4). For any sheaf $\mathcal{F}$ on $Y$, denoting by the same symbol its pull-backs to $\mathcal{X}_{1} \times Y$, $X \times \mathbf{P} \times Y$ and $X \times Y$, and using the projection formula, we obtain

$$
\alpha_{*}\left(j_{*} \mathcal{E} \otimes \mathcal{F}\right)=\alpha_{*} j_{*} \mathcal{E} \otimes \mathcal{F}=\beta_{*} i_{*} \mathcal{E} \otimes \mathcal{F}=\beta_{*}\left(i_{*} \mathcal{E} \otimes \mathcal{F}\right) .
$$

Since $\alpha_{*}$ and $\beta_{*}$ are exact it follows that the Tor-amplitude of $j_{*} \mathcal{E}$ over $Y$ is equal to the Tor-amplitude of $i_{*} \mathcal{E}$ over $Y$, and the latter is finite since $i_{*} \mathcal{E}$ admits a finite locally free resolution (2) on $X \times Y$. Since $\mathcal{X}_{1} \times Y$ is smooth over $Y$ by lemma 3.2, we conclude by lemma D.43.

Lemma 3.6. The functor $\Phi_{\mathcal{E}_{1}^{\# 0}}$ is left adjoint to $\Phi_{\mathcal{E}_{1}}$.

Proof: Note that $\mathcal{E}_{1}$ is a perfect complex by lemma 3.5, and its support $Q(X, Y) \cong \mathcal{X}_{1} \times_{\mathbf{P}} Y$ is projective over $\mathcal{X}_{1}$ by (C.4) and over $Y$ by lemma 3.2. Therefore, according to lemma 2.5 it suffices to verify that $\mathcal{E}_{1}^{\# 0} \cong \operatorname{R\mathcal {H}om}\left(\mathcal{E}_{1}, \omega_{\mathcal{X}_{1}}\left[\operatorname{dim} \mathcal{X}_{1}\right]\right)$. Let $q_{1}$ and $q$ be the projections $\mathcal{X}_{1} \times Y \rightarrow Y$ and $X \times Y \rightarrow Y$. It is clear that $q \circ i=q_{1} \circ j$. Using the duality theorem and the functoriality of the twisted pullback we deduce

$$
\begin{aligned}
& \operatorname{RHom}\left(\mathcal{E}_{1}, \omega_{\mathcal{X}_{1}}\left[\operatorname{dim} \mathcal{X}_{1}\right]\right) \cong \operatorname{RHom}\left(j_{*} \mathcal{E}, q_{1}^{!} \mathcal{O}_{Y}\right) \cong j_{*} \operatorname{RHom}\left(\mathcal{E}, j^{!} q_{1}^{!} \mathcal{O}_{Y}\right) \cong j_{*} \operatorname{RHom}\left(\mathcal{E}, i^{!} q^{!} \mathcal{O}_{Y}\right) \cong \\
& \cong j_{*} \operatorname{RHom}\left(\mathcal{E}, i^{!} \omega_{X}[\operatorname{dim} X]\right) \cong j_{*} \operatorname{RHom}\left(\mathcal{E}, \omega_{X}(1,1)[\operatorname{dim} X-1]\right) \cong j_{*} \mathcal{E}^{*}(1-i, 1)[\operatorname{dim} X-1],
\end{aligned}
$$

since $\omega_{X} \cong \mathcal{O}_{X}(-i)$ by $($ C.2).

Twisting (7) by $\mathcal{O}(1+t-i, 1)$ and taking into account (5), we obtain an exact triangle

$$
E_{2}^{*}(t-i) \otimes F_{2}^{*} \stackrel{e^{*}}{\rightarrow} E_{1}^{*}(t-i) \otimes F_{1}^{*} \rightarrow i_{*} \mathcal{E}^{\# t}
$$


Consider the following diagram

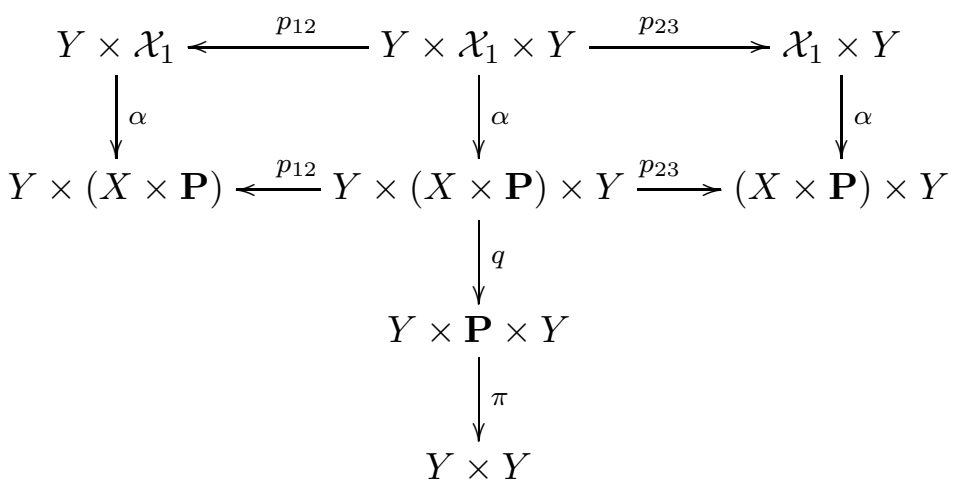

where $\pi$ is the projection along $\mathbf{P}$. Consider the following objects in $\mathcal{D}(Y \times \mathbf{P} \times Y)$ :

$$
\mathcal{C}_{t}=q_{*} \alpha_{*}\left(p_{12}^{*} \mathcal{E}_{1}^{\# t} \otimes p_{23}^{*} \mathcal{E}_{1}\right), \quad \quad \tilde{\mathcal{C}}_{t}=q_{*}\left(\alpha_{*} p_{12}^{*} \mathcal{E}_{1}^{\# t} \otimes \alpha_{*} p_{23}^{*} \mathcal{E}_{1}\right)
$$

Lemma 3.7. We have $\mathcal{E}_{1} \circ \mathcal{E}_{1}^{\# t} \cong \pi_{*} \mathcal{C}_{t} \in \mathcal{D}(Y \times Y)$.

Proof: Use the definition of the convolution and note that $p_{13}=\pi \circ q \circ \alpha$.

Lemma 3.8. There exists $N \in \mathbb{Z}$ such that $\mathcal{C}_{t} \in \mathcal{D}^{[-N, 0]}(Y \times \mathbf{P} \times Y)$ for all $t \in \mathbb{Z}$.

Proof: Since $\mathcal{E}_{1}$ is perfect by lemma 3.5 , it follows that $p_{23}^{*} \mathcal{E}_{1}$ is perfect as well. Hence $p_{12}^{*} \mathcal{E}_{1}^{\# t} \otimes p_{23}^{*} \mathcal{E}_{1}$ is cohomologically bounded. Moreover, since $\mathcal{E}_{1}^{\# t} \cong \mathcal{E}_{1}^{\# 0} \otimes\left(\mathcal{L}^{* \otimes t} \otimes \mathcal{O}_{X}(t)\right)$, the left bound doesn't depend on $t$. Thus, there exists $N \in \mathbb{Z}$, such that $p_{12}^{*} \mathcal{E}_{1}^{\# t} \otimes p_{23}^{*} \mathcal{E}_{1} \in \mathcal{D}^{\geq-N}\left(Y \times \mathcal{X}_{1} \times Y\right)$. On the other hand, $p_{12}^{*} \mathcal{E}_{1}^{\# t} \in \mathcal{D}^{1-\operatorname{dim} X}\left(Y \times \mathcal{X}_{1} \times Y\right)$ by (5) and lemma 3.4 , and $p_{23}^{*} \mathcal{E}_{1} \in \mathcal{D}^{0}\left(Y \times \mathcal{X}_{1} \times Y\right)$ by (5). Hence $p_{12}^{*} \mathcal{E}_{1}^{\# t} \otimes p_{23}^{*} \mathcal{E}_{1} \in \mathcal{D}^{[-N, 1-\operatorname{dim} X]}\left(Y \times \mathcal{X}_{1} \times Y\right)$. Since $q \circ \alpha$ is projective and has relative $\operatorname{dimension} \operatorname{dim} X-1$ by lemma 3.2 , we are done.

Lemma 3.9. We have an exact triangle

$$
\tilde{\mathcal{C}}_{t} \rightarrow \mathcal{C}_{t} \rightarrow \mathcal{C}_{t-1}[2]
$$

in $\mathcal{D}^{b}(Y \times \mathbf{P} \times Y)$.

Proof: Since $\alpha: \mathcal{X}_{1} \rightarrow X \times \mathbf{P}$ is a divisorial embedding, and $\mathcal{X}_{1}$ is a zero locus of a section of the bundle $\mathcal{O}_{X}(1) \otimes \mathcal{L}^{*}$, we have an exact triangle

$$
\alpha^{*} \alpha_{*} \mathcal{F} \rightarrow \mathcal{F} \rightarrow \mathcal{F} \otimes\left(\mathcal{O}_{X}(-1) \otimes \mathcal{L}\right)[2]
$$

for any object $\mathcal{F}$ on $Y \times \mathcal{X}_{1} \times Y$. Taking $\mathcal{F}=p_{23}^{*} \mathcal{E}_{1}$, tensoring with $p_{12}^{*} \mathcal{E}_{1}^{\# t}$, applying $q_{*} \alpha_{*}$ and taking into account the projection formula $\alpha_{*}\left(p_{12}^{*} \mathcal{E}_{1}^{\# t} \otimes \alpha^{*} \alpha_{*} p_{23}^{*} \mathcal{E}_{1}\right) \cong \alpha_{*} p_{12}^{*} \mathcal{E}_{1}^{\# t} \otimes \alpha_{*} p_{23}^{*} \mathcal{E}_{1}$, and the definition of $\mathcal{C}_{t}$ and $\tilde{\mathcal{C}}_{t}(10)$, we obtain $(11)$

Denote by $\beta^{\prime}: Y \rightarrow Y \times \mathbf{P}$ the composition of $\beta$ with the transposition $\mathbf{P} \times Y \rightarrow Y \times \mathbf{P}$. Consider the $\operatorname{maps}_{Y} \times \beta, \beta^{\prime} \times \operatorname{id}_{Y}: Y \times Y \rightarrow Y \times \mathbf{P} \times Y$ and denote them by $\beta$ and $\beta^{\prime}$ for brevity. Let

$$
\mathfrak{D}:=\beta_{*}^{\prime} \mathcal{O}_{Y \times Y} \otimes \beta_{*} \mathcal{O}_{Y \times Y} \in \mathcal{D}(Y \times \mathbf{P} \times Y) .
$$

Lemma 3.10. We have $\mathfrak{D} \in \mathcal{D}^{\left[\operatorname{codim} Y \times_{\mathbf{P}} Y-\operatorname{dim} \mathbf{P}, 0\right]}(Y \times \mathbf{P} \times Y)$. Moreover, $\mathfrak{D}$ is supported scheme-

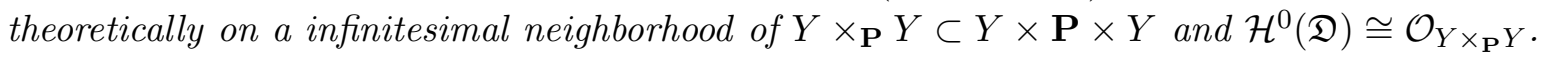


Proof: Consider a diagram

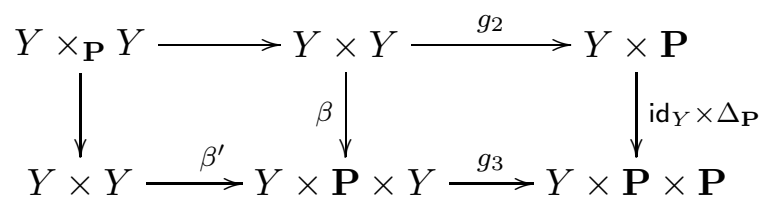

where $g_{2}=\mathrm{id}_{Y} \times g, g_{3}=\mathrm{id}_{Y} \times \mathrm{id}_{\mathbf{P}} \times g$ and $\Delta_{\mathbf{P}}$ is the diagonal embedding $\mathbf{P} \rightarrow \mathbf{P} \times \mathbf{P}$. Both squares are cartesian. Moreover, since $\mathbf{P}$ is smooth the right vertical arrow is a locally complete intersection embedding. Since codim $Y \times \mathbf{P} \times Y(Y \times Y)=\operatorname{codim}_{Y \times \mathbf{P} \times \mathbf{P}}(Y \times \mathbf{P})=\operatorname{dim} \mathbf{P}$, the right square is exact cartesian by corollary 2.27. Further, locally over $Y \times \mathbf{P} \times \mathbf{P}$ we can represent $Y \times \mathbf{P}$ as the zero locus of a regular section $s$ of a vector bundle on $Y \times \mathbf{P} \times \mathbf{P}$ of rank equal to $\operatorname{dim} \mathbf{P}$, hence we have a local representation $\left(\operatorname{id}_{Y} \times \Delta_{\mathbf{P}}\right)_{*} \mathcal{O}_{Y \times \mathbf{P}} \cong \operatorname{Kosz}_{Y \times \mathbf{P} \times \mathbf{P}}(s)$. It follows that

$$
\beta_{*} \mathcal{O}_{Y \times Y} \cong \beta_{*} g_{2}^{*} \mathcal{O}_{Y \times \mathbf{P}} \cong g_{3}^{*}\left(\operatorname{id}_{Y} \times \Delta_{\mathbf{P}}\right)_{*} \mathcal{O}_{Y \times \mathbf{P}} \cong g_{3}^{*} \operatorname{Kosz}_{Y \times \mathbf{P} \times \mathbf{P}}(s)
$$

and

$$
\mathfrak{D}=\beta_{*}^{\prime} \mathcal{O}_{Y \times Y} \otimes \beta_{*} \mathcal{O}_{Y \times Y} \cong \beta_{*}^{\prime} \beta^{\prime *} \beta_{*} \mathcal{O}_{Y \times Y} \cong \beta_{*}^{\prime} \beta^{\prime *} g_{3}^{*} \operatorname{Kosz}_{Y \times \mathbf{P} \times \mathbf{P}}(s) \cong \beta_{*}^{\prime} \operatorname{Kosz}_{Y \times Y}\left(\beta^{\prime *} g_{3}^{*} s\right) .
$$

It remains to note that the zero locus of the section ${\beta^{\prime *}}^{*} g_{3}^{*}$ on $Y \times Y$ coincides with $Y \times_{\mathbf{P}} Y$ and the proof concludes with lemma 2.16.

Consider on $Y \times Y$ the objects $\mathcal{T}, \mathcal{T}^{*} \in \mathcal{D}(Y \times Y)$ defined by the following exact triangles

$$
\begin{array}{r}
F_{2}^{*} \otimes F_{2} \oplus F_{1}^{*} \otimes F_{1} \stackrel{\operatorname{ad}(\phi)}{\operatorname{ad}(\phi)} W^{*} \otimes F_{1}^{*} \otimes F_{2} \longrightarrow F_{2}^{*} \otimes F_{2} \oplus F_{1}^{*} \otimes F_{1} \longrightarrow \mathcal{T}^{*} . \\
W \otimes F_{2}^{*} \otimes F_{1} \longrightarrow \frac{\operatorname{Tod}}{\longrightarrow}
\end{array}
$$

Lemma 3.11. We have $\tilde{\mathcal{C}}_{t} \in \mathcal{D}^{[-1-B, 1]}(Y \times \mathbf{P} \times Y)$ for all $t \in \mathbb{Z}$. Moreover,

$$
\tilde{\mathcal{C}}_{t}= \begin{cases}\pi^{*} \mathcal{T}^{*} \otimes \mathfrak{D}, & \text { for } t=0 \\ 0, & \text { for } 1 \leq t \leq i-1 \\ \pi^{*} \mathcal{T} \otimes \mathcal{L}^{* \otimes i} \otimes \mathfrak{D}[\operatorname{dim} X-1], & \text { for } t=i\end{cases}
$$

In particular, $\tilde{\mathcal{C}}_{i} \in \mathcal{D}^{[-B, 1-\operatorname{dim} X]}(Y \times \mathbf{P} \times Y)$ and $\mathcal{H}^{0}\left(\tilde{\mathcal{C}}_{0}\right) \neq 0$.

Proof: Consider the diagram

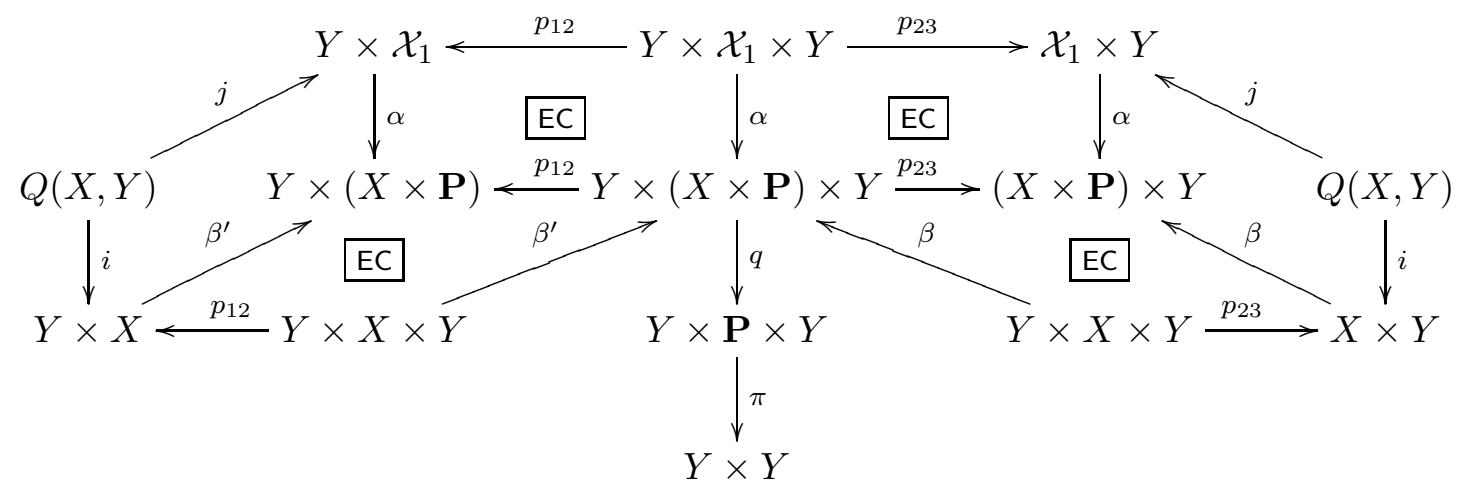

and note that the squares marked with $\mathrm{EC}$ are exact cartesian by corollary 2.27. Hence we have,

$$
\alpha_{*} p_{23}^{*} \mathcal{E}_{1} \cong p_{23}^{*} \alpha_{*} \mathcal{E}_{1}=p_{23}^{*} \alpha_{*} j_{*} \mathcal{E} \cong p_{23}^{*} \beta_{*} i_{*} \mathcal{E} \cong \beta_{*} p_{23}^{*} i_{*} \mathcal{E}
$$

and similarly $\alpha_{*} p_{12}^{*} \mathcal{E}_{1}^{\# t} \cong \beta_{*}^{\prime} p_{12}^{*} i_{*} \mathcal{E}^{\# t} \otimes \mathcal{L}^{* \otimes t}[\operatorname{dim} X-1]$. Therefore,

$$
\tilde{\mathcal{C}_{t}} \cong q_{*}\left(\beta_{*}^{\prime} p_{12}^{*} i_{*} \mathcal{E}^{\# t} \otimes \mathcal{L}_{26}^{* \otimes t}[\operatorname{dim} X-1] \otimes \beta_{*} p_{23}^{*} i_{*} \mathcal{E}\right) .
$$


Applying $\beta_{*} p_{23}^{*}$ to $(2)$ and $\beta_{*}^{\prime} p_{12}^{*}$ to $(8)$ we obtain exact triangles

$$
\begin{array}{lll}
\beta_{*}\left(\mathcal{O} \otimes E_{1} \otimes F_{1}\right) & \stackrel{e}{\longrightarrow} \beta_{*}\left(\mathcal{O} \otimes E_{2} \otimes F_{2}\right) & \rightarrow \beta_{*} p_{23}^{*} i_{*} \mathcal{E}, \\
\beta_{*}^{\prime}\left(F_{2}^{*} \otimes E_{2}^{*}(t-i) \otimes \mathcal{O}\right) \stackrel{e^{*}}{\longrightarrow} \beta_{*}^{\prime}\left(F_{1}^{*} \otimes E_{1}^{*}(t-i) \otimes \mathcal{O}\right) \rightarrow \beta_{*}^{\prime} p_{12}^{*} i_{*} \mathcal{E}^{\# t} .
\end{array}
$$

So, roughly speaking we have a resolution

$$
\left\{\tilde{\mathcal{C}}_{t}^{2,1} \rightarrow \tilde{\mathcal{C}}_{t}^{1,1} \oplus \tilde{\mathcal{C}}_{t}^{2,2} \rightarrow \tilde{\mathcal{C}}_{t}^{1,2}\right\} \cong \tilde{\mathcal{C}}_{t}
$$

where

$$
\begin{aligned}
\tilde{\mathcal{C}}_{t}^{k, l}:=q_{*}\left(\beta _ { * } ^ { \prime } \left(F_{k}^{*} \otimes\right.\right. & \left.\left.E_{k}^{*}(t-i) \otimes \mathcal{O}\right) \otimes \mathcal{L}^{* \otimes t}[\operatorname{dim} X-1] \otimes \beta_{*}\left(\mathcal{O} \otimes E_{l} \otimes F_{l}\right)\right) \cong \\
& \cong q_{*}\left(\left(F_{k}^{*} \otimes\left(E_{k}^{*}(t-i) \otimes E_{l}\right) \otimes \mathcal{L}^{* \otimes t} \otimes F_{l}\right) \otimes \beta_{*}^{\prime} \mathcal{O}_{Y \times X \times Y} \otimes \beta_{*} \mathcal{O}_{Y \times X \times Y}\right)[\operatorname{dim} X-1]
\end{aligned}
$$

for $k, l=1,2$ (more rigorously, we should say that there are three exact triangles, obtained by tensoring the first triangle of (15) by terms of the second triangle). To compute $\tilde{\mathcal{C}}_{t}^{k, l}$ we note that the squares in the diagram

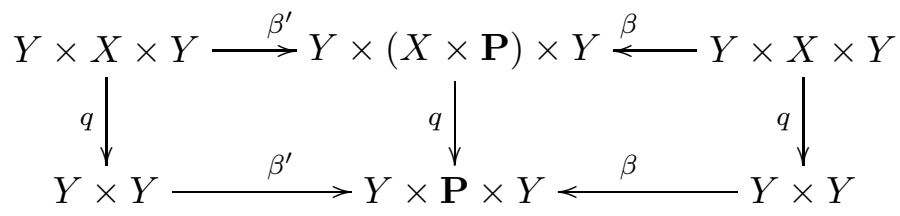

are exact cartesian by corollary 2.23 , hence

$$
\beta_{*}^{\prime} \mathcal{O}_{Y \times X \times Y} \otimes \beta_{*} \mathcal{O}_{Y \times X \times Y} \cong \beta_{*}^{\prime} q^{*} \mathcal{O}_{Y \times Y} \otimes \beta_{*} q^{*} \mathcal{O}_{Y \times Y} \cong q^{*} \beta_{*}^{\prime} \mathcal{O}_{Y \times Y} \otimes q^{*} \beta_{*} \mathcal{O}_{Y \times Y} \cong q^{*} \mathfrak{D} .
$$

Substituting this into the formula for $\tilde{\mathcal{C}}_{t}^{k, l}$ we deduce

$$
\left.\tilde{\mathcal{C}}_{t}^{k, l} \cong\left(F_{k}^{*} \otimes\left(H^{\bullet}\left(X, E_{k}^{*}(t-i) \otimes E_{l}\right) \otimes \mathcal{L}^{* \otimes t}\right) \otimes F_{l}\right) \otimes \mathfrak{D}\right)[\operatorname{dim} X-1]
$$

Since $\mathfrak{D} \in \mathcal{D}^{\left[\operatorname{codim}_{Y \times Y} Y \times_{\mathbf{P}} Y-\operatorname{dim} \mathbf{P}, 0\right]}(Y \times \mathbf{P} \times Y)$ by lemma 3.10, $H^{\bullet}\left(X, E_{k}^{*}(t-i) \otimes E_{l}\right) \in \mathcal{D}^{[0, \operatorname{dim} X]}(\mathcal{A} b)$, and $\left(\operatorname{codim}_{Y \times Y} Y \times_{\mathbf{P}} Y-\operatorname{dim} \mathbf{P}\right)-(\operatorname{dim} X-1)=1-B$, by $(6)$, we deduce $\tilde{\mathcal{C}}_{t}^{k, l} \in \mathcal{D}^{[1-B, 1]}(Y \times \mathbf{P} \times Y)$, for all $t \in \mathbb{Z}, k, l=1,2$. Hence, looking at (16) we see that $\tilde{\mathcal{C}}_{t} \in \mathcal{D}^{[-1-B, 1]}(Y \times \mathbf{P} \times Y)$ for all $t \in \mathbb{Z}$. Moreover, for $1 \leq t \leq i-1$ we have $H^{\bullet}\left(X, E_{k}^{*}(t-i) \otimes E_{l}\right) \cong \operatorname{Hom}^{\bullet}\left(E_{k}, E_{l}(t-i)\right)=0$, hence $\tilde{\mathcal{C}}_{t}^{k, l}=0$ for $k, l=1,2$, therefore $\tilde{\mathcal{C}_{t}}=0$ for $1 \leq t \leq i-1$.

Finally, for $t=0$ we have

$$
H^{\bullet}\left(X, E_{k}^{*}(t-i) \otimes E_{l}\right) \cong \operatorname{Hom}^{\bullet}\left(E_{k}, E_{l}(-i)\right) \cong \begin{cases}\mathrm{k}[-\operatorname{dim} X], & \text { for } k=l \\ W[-\operatorname{dim} X], & \text { for } l=1, k=2 \\ 0, & \text { for } l=2, k=1\end{cases}
$$

hence $\tilde{\mathcal{C}}_{0}^{2,1}=\pi^{*}\left(W \otimes F_{2}^{*} \otimes F_{1}\right) \otimes \mathfrak{D}[-1], \tilde{\mathcal{C}}_{0}^{1,1}=\pi^{*}\left(F_{1}^{*} \otimes F_{1}\right) \otimes \mathfrak{D}[-1], \tilde{\mathcal{C}}_{0}^{2,2}=\pi^{*}\left(F_{2}^{*} \otimes F_{2}\right) \otimes \mathfrak{D}[-1]$, and (16) gives a triangle

$$
\pi^{*}\left(W \otimes F_{2}^{*} \otimes F_{1}\right) \otimes \mathfrak{D} \rightarrow \pi^{*}\left(F_{2}^{*} \otimes F_{2} \oplus F_{1}^{*} \otimes F_{1}\right) \otimes \mathfrak{D} \rightarrow \tilde{\mathcal{C}_{0}} .
$$

whereof we deduce $\tilde{\mathcal{C}_{0}} \cong \pi^{*} \mathcal{T}^{*} \otimes \mathfrak{D}$. Note also that $\mathcal{H}^{0}\left(\mathcal{T}^{*}\right)=$ Coker ad $(\phi) \cong \Delta_{Y *} \mathcal{O}_{Y}$ by condition (C.5), $\mathcal{H}^{0}(\mathfrak{D}) \cong \mathcal{O}_{Y \times_{\mathbf{P}} Y}$ by lemma 3.10 , so that $\mathcal{H}^{0}\left(\tilde{\mathcal{C}}_{0}\right) \neq 0$.

Similarly, for $t=i$ we have

$$
H^{\bullet}\left(X, E_{k}^{*}(t-i) \otimes E_{l}\right) \cong \operatorname{Hom}^{\bullet}\left(E_{k}, E_{l}\right) \cong \begin{cases}\mathrm{k}, & \text { for } k=l \\ W^{*}, & \text { for } l=2, k=1 \\ 0, & \text { for } l=1, k=2\end{cases}
$$


hence $\tilde{\mathcal{C}}_{i}^{1,1}=\pi^{*}\left(F_{1}^{*} \otimes F_{1}\right) \otimes \mathcal{L}^{* \otimes i} \otimes \mathfrak{D}[\operatorname{dim} X-1], \tilde{\mathcal{C}}_{i}^{2,2}=\pi^{*}\left(F_{2}^{*} \otimes F_{2}\right) \otimes \mathcal{L}^{* \otimes i} \otimes \mathfrak{D}[\operatorname{dim} X-1]$, and $\tilde{\mathcal{C}}_{i}^{1,2}=\pi^{*}\left(W \otimes F_{1}^{*} \otimes F_{2}\right) \otimes \mathcal{L}^{* \otimes i} \otimes \mathfrak{D}[\operatorname{dim} X-1]$. So, (16) gives a triangle

$$
\pi^{*}\left(F_{2}^{*} \otimes F_{2} \oplus F_{1}^{*} \otimes F_{1}\right) \otimes \mathcal{L}^{* \otimes i} \otimes \mathfrak{D}[\operatorname{dim} X-1] \stackrel{\left(e^{*}, e\right)}{\longrightarrow} \pi^{*}\left(W^{*} \otimes F_{1}^{*} \otimes F_{2}\right) \otimes \mathcal{L}^{* \otimes i} \otimes \mathfrak{D}[\operatorname{dim} X-1] \rightarrow \tilde{\mathcal{C}}_{i} .
$$

whereof we deduce $\tilde{\mathcal{C}}_{i} \cong \pi^{*} \mathcal{T} \otimes \mathcal{L}^{* \otimes i} \otimes \mathfrak{D}[\operatorname{dim} X-1]$. In particular, $\tilde{\mathcal{C}}_{i} \in \mathcal{D}^{[-B, 1-\operatorname{dim} X]}(Y \times \mathbf{P} \times Y)$ since $\tilde{\mathcal{C}}_{i}^{2,1}=0$.

\subsection{Proof of theorem 3.1.}

Proposition 3.12. We have $B \geq 2 i-1$. Moreover, if $B=2 i-1$, then

$$
\mathcal{C}_{0}=\mathcal{C}_{1}[-2]=\cdots=\mathcal{C}_{i-1}[2-2 i] \cong \mathcal{H}^{0}\left(\tilde{\mathcal{C}}_{0}\right)
$$

Proof: First of all, we note that for all $t \in \mathbb{Z}$ we have

$$
\mathcal{C}_{t} \in \mathcal{D}^{[-B, 0]}(Y \times \mathbf{P} \times Y) .
$$

Indeed, it follows from triangle (11) and lemma 3.11 that for any $l \leq-1-B$ we have an exact sequence

$$
0=\mathcal{H}^{l-2}\left(\tilde{\mathcal{C}}_{t}\right) \rightarrow \mathcal{H}^{l-2}\left(\mathcal{C}_{t}\right) \rightarrow \mathcal{H}^{l-2}\left(\mathcal{C}_{t-1}[2]\right) \rightarrow \mathcal{H}^{l-1}\left(\tilde{\mathcal{C}}_{t}\right)=0 .
$$

Therefore, $\mathcal{H}^{l}\left(\mathcal{C}_{t-1}\right)=\mathcal{H}^{l-2}\left(\mathcal{C}_{t-1}[2]\right) \cong \mathcal{H}^{l-2}\left(\mathcal{C}_{t}\right)$. So, if $\mathcal{H}^{l}\left(\mathcal{C}_{t}\right)=0$ for $l \leq-1-B$ then $\mathcal{H}^{l-2 s}\left(\mathcal{C}_{t+s}\right) \neq 0$ for all $s$ which contradicts the claim of lemma 3.8.

Further, using triangle (11) and lemma 3.11 for $t=i-1, \ldots, 1$ we see that

$$
\mathcal{C}_{i-1}=\mathcal{C}_{i-2}[2]=\cdots=\mathcal{C}_{0}[2 i-2] .
$$

Therefore, $\mathcal{C}_{i-1}[2]=\mathcal{C}_{0}[2 i]$, hence

$$
\mathcal{C}_{i-1}[2] \in \mathcal{D}^{\leq-2 i}
$$

by lemma 3.8. Using triangle (11) for $t=i:\left\{\tilde{\mathcal{C}}_{i} \rightarrow \mathcal{C}_{i} \rightarrow \mathcal{C}_{i-1}[2]\right\}$ and recalling that $\tilde{\mathcal{C}}_{i} \in \mathcal{D}^{\geq-B}$ by lemma 3.11 and $\mathcal{C}_{i} \in \mathcal{D}^{\geq-B}$ by (17), we deduce

$$
\mathcal{C}_{i-1}[2] \in \mathcal{D}^{\geq-1-B} .
$$

Now, if $B<2 i-1$, then $-2 i<-1-B$, and comparing (18) with (19) we deduce $\mathcal{C}_{i-1}=0$. Hence $\mathcal{C}_{0}=\mathcal{C}_{i-1}[2-2 i]=0$ as well. But then the triangle $(11)$ for $t=0: \quad\left\{\tilde{\mathcal{C}}_{0} \rightarrow \mathcal{C}_{0} \rightarrow \mathcal{C}_{-1}[2]\right\}$ implies $\mathcal{C}_{-1}=\tilde{\mathcal{C}}_{0}[-1]$, hence $\mathcal{H}^{1}\left(\mathcal{C}_{-1}\right)=\mathcal{H}^{0}\left(\tilde{\mathcal{C}}_{0}\right) \neq 0$ by lemma 3.11 , which contradicts lemma 3.8. Therefore, $B \geq 2 i-1$.

If $B=2 i-1$, then $-2 i=-1-B$, and comparing (18) with (19) we deduce that $\mathcal{C}_{i-1}[2] \in \mathcal{D}^{-2 i}$. Hence $\mathcal{C}_{0}=\mathcal{C}_{i-1}[2-2 i] \in D^{0}$. Since by lemma 3.8 we have $\mathcal{C}_{-1}[2] \in \mathcal{D}^{\leq-2}$ the triangle (11) for $t=0$ implies that $\mathcal{C}_{0}=\mathcal{H}^{0}\left(\tilde{\mathcal{C}}_{0}\right)$.

Corollary 3.13. If $B=2 i-1$ then we have

$$
\pi_{*} \mathcal{C}_{0}=\pi_{*} \mathcal{C}_{1}[-2]=\cdots=\pi_{*} \mathcal{C}_{i-1}[2-2 i] \cong \Delta_{Y *} \mathcal{O}_{Y}
$$

Proof: Note that

$$
\pi_{*} \tilde{\mathcal{C}}_{0} \cong \pi_{*}\left(\pi^{*} \mathcal{T}^{*} \otimes \mathfrak{D}\right) \cong \mathcal{T}^{*} \otimes \pi_{*} \mathfrak{D} .
$$

On the other hand, by lemma 3.10 the object $\mathfrak{D}$ is supported scheme-theoretically on a infinitesimal neighborhood of $Y{ }_{\mathbf{P}} Y \subset Y \times \mathbf{P} \times Y$. But the restriction of $\pi: Y \times \mathbf{P} \times Y \rightarrow Y \times Y$ to the infinitesimal neighborhood of $Y \times_{\mathbf{P}} Y \subset Y \times \mathbf{P} \times Y$ is finite, hence

$$
\pi_{*} \mathcal{C}_{0} \cong \pi_{*} \mathcal{H}^{0}\left(\tilde{\mathcal{C}}_{0}\right) \cong \mathcal{H}^{0}\left(\pi_{*} \tilde{\mathcal{C}}_{0}\right) \cong \mathcal{H}^{0}\left(\mathcal{T}^{*} \otimes \pi_{*} \mathfrak{D}\right)
$$


Finally, we have $\mathcal{H}^{0}\left(\mathcal{T}^{*}\right)=\operatorname{Coker}\left(W \otimes F_{2}^{*} \otimes F_{1} \stackrel{\operatorname{ad}(\phi)}{\longrightarrow} F_{2}^{*} \otimes F_{2} \oplus F_{1}^{*} \otimes F_{1}\right) \cong \Delta_{Y *} \mathcal{O}_{Y}$ by (13), and $\mathcal{H}^{0}\left(\pi_{*} \mathfrak{D}\right) \cong \mathcal{H}^{0}\left(\pi_{*} \mathcal{O}_{Y \times_{\mathbf{P}} Y}\right) \cong \mathcal{O}_{Y \times_{\mathbf{P}} Y}$ by lemma 3.10 , hence

$$
\pi_{*} \mathcal{C}_{0} \cong \mathcal{H}^{0}\left(\Delta_{Y *} \mathcal{O}_{Y} \otimes \mathcal{O}_{Y \times_{\mathbf{P}} Y}\right) \cong \Delta_{Y *} \mathcal{O}_{Y}
$$

since $\Delta_{Y}(Y) \subset Y \times_{\mathbf{P}} Y$.

Corollary 3.14. If $B=2 i-1$ then the functor $\Phi_{\mathcal{E}_{1}^{\# t}} \circ \Phi_{\mathcal{E}_{1}}$ is isomorphic to the shift by [2t] for any $t=0,1, \ldots, i-1$.

Proof: Apply lemma 3.7 and corollary 3.13.

\section{Proof of theorem 3.1.}

We have $B \geq 2 i-1$ by proposition 3.12 . Assume that $B=2 i-1$. Since $\Phi_{\mathcal{E}_{1}^{\# 0}}$ is left adjoint to $\Phi_{\mathcal{E}_{1}}$ by lemma 3.6, it follows from corollary 3.14 that $\Phi_{\mathcal{E}_{1}}$ is fully faithful and it remains to show that $Y$ is smooth. To this end we note that $\mathcal{X}_{1}$ is smooth by lemma 3.2, hence $\mathcal{D}^{b}\left(\mathcal{X}_{1}\right)$ is Ext-bounded by lemma D.22. On the other hand, as we have shown above $\mathcal{D}^{b}(Y)$ embeds fully and faithfully into $\mathcal{D}^{b}\left(\mathcal{X}_{1}\right)$, hence $\mathcal{D}^{b}(Y)$ is also Ext-bounded. Applying again lemma D.22 we deduce that $Y$ is smooth.

We will need also the following

Proposition 3.15. If $B=2 i-1$ then

$$
\Phi_{1}\left(\mathcal{D}^{b}(Y)\right) \subset\left\langle E_{1}(1) \otimes \mathcal{D}^{b}(\mathbf{P}), \ldots, E_{2}(i-1) \otimes \mathcal{D}^{b}(\mathbf{P})\right\rangle^{\perp} .
$$

Proof: We must check that $\operatorname{Hom}\left(E_{s}(k) \otimes F, \Phi_{\mathcal{E}_{1}}(G)\right)=0$ for all $F \in \mathcal{D}^{b}(\mathbf{P}), G \in \mathcal{D}^{b}(Y)$. Note that $E_{s}(k) \otimes F \cong \alpha^{*}\left(E_{s}(k) \otimes F\right)$ and $\operatorname{Hom}\left(\alpha^{*}\left(E_{s}(k) \otimes F\right), \Phi_{\mathcal{E}_{1}}(G)\right) \cong \operatorname{Hom}\left(E_{s}(k) \otimes F, \alpha_{*} \Phi_{\mathcal{E}_{1}}(G)\right)$. Further, $\alpha_{*} \Phi_{\mathcal{E}_{1}}$ is a kernel functor with kernel $\alpha_{*} j_{*} \mathcal{E} \cong \beta_{*} i_{*} \mathcal{E}$ and

$$
\begin{aligned}
\operatorname{Hom}_{X \times \mathbf{P}}\left(E_{s}(k) \otimes F,\right. & \left.\Phi_{\beta_{*} i_{*} \mathcal{E}}(G)\right)=\operatorname{Hom}_{X \times \mathbf{P}}\left(E_{s}(k) \otimes F, p_{*}\left(\beta_{*} i_{*} \mathcal{E} \otimes q^{*} G\right)\right) \cong \\
& \cong \operatorname{Hom}_{X \times \mathbf{P} \times Y}\left(E_{s}(k) \otimes F \otimes G^{*}, \beta_{*} i_{*} \mathcal{E}\right) \cong \operatorname{Hom}_{X \times Y}\left(E_{s}(k) \otimes\left(g^{*} F \otimes G^{*}\right), i_{*} \mathcal{E}\right)
\end{aligned}
$$

and the last space is zero because $i_{*} \mathcal{E} \in\left\langle E_{1} \otimes \mathcal{D}^{b}(Y), E_{2} \otimes \mathcal{D}^{b}(Y)\right\rangle$ and by condition (C.3) we have $E_{1}, E_{2} \in\left\langle E_{1}(1), \ldots, E_{2}(i-1)\right\rangle^{\perp}$.

\section{Semiorthogonal DeCompositions}

In this section we conclude the proof of theorem 1.2. In fact, instead of individual linear sections of $X$ and $Y$ we consider the universal families of linear sections and prove a similar statements for them. After that theorem 1.2 follows by a faithful base change argument.

4.1. Universal families of linear sections. All $r$-dimensional subspaces $L \subset V^{*}$ are parameterized by the Grassmannian $\operatorname{Gr}\left(r, V^{*}\right)$. Those of them, for which the linear sections $X_{L}=X \times_{\mathbb{P}(V)} \mathbb{P}\left(L^{\perp}\right)$ and $Y_{L}=Y \times_{\mathbb{P}\left(V^{*}\right)} \mathbb{P}(L)$ are compact are parameterized by an open subset of the Grassmannian $\mathbf{P}_{r} \subset \operatorname{Gr}\left(r, V^{*}\right)$ consisting of all $r$-dimensional subspaces $L \subset V^{*}$ such that $L \cap \mathbf{Z}=\emptyset$. Let $\mathcal{L}_{r}$ be the pullback of the tautological rank $r$ subbundle of the Grassmannian $\operatorname{Gr}\left(r, V^{*}\right)$ to $\mathbf{P}_{r}$ and let $\mathcal{L}_{r}^{\perp}:=\left(V^{*} \otimes \mathcal{O}_{\mathbf{P}_{r}} / \mathcal{L}_{r}\right)^{*} \subset$ $V \otimes \mathcal{O}_{\mathbf{P}_{r}}$, be the orthogonal subbundle. Then the subvarieties

$$
\begin{aligned}
& \mathcal{X}_{r}=\left(X \times \mathbf{P}_{r}\right) \times_{\mathbb{P}(V) \times \mathbf{P}_{r}} \mathbb{P}_{\mathbf{P}_{r}}\left(\mathcal{L}_{r}^{\perp}\right) \subset X \times \mathbf{P}_{r}, \\
& \mathcal{Y}_{r}=\left(Y \times \mathbf{P}_{r}\right) \times_{\mathbb{P}\left(V^{*}\right) \times \mathbf{P}_{r}} \mathbb{P}_{\mathbf{P}_{r}}\left(\mathcal{L}_{r}\right) \subset Y \times \mathbf{P}_{r},
\end{aligned}
$$

are the universal families of compact linear sections of $X$ and $Y$.

Consider the fiber product $\mathcal{X}_{r} \times{ }_{\mathbf{P}_{r}} \mathcal{Y}_{r}$ and the projection $\pi_{r}: \mathcal{X}_{r} \times_{\mathbf{P}_{r}} \mathcal{Y}_{r} \rightarrow X \times Y$. Since for any vector subspace $L \subset V^{*}$ the product $\mathbb{P}\left(L^{\perp}\right) \times \mathbb{P}(L)$ is contained in the incidence quadric $Q \subset \mathbb{P}(V) \times \mathbb{P}\left(V^{*}\right)$ it follows that $\pi_{r}$ factors via a map $\zeta_{r}: \mathcal{X}_{r} \times \mathbf{P}_{r} \mathcal{Y}_{r} \rightarrow Q(X, Y) \subset X \times Y$. 
Consider the object $\mathcal{E}_{r}=\zeta_{r}^{*} \mathcal{E} \in \mathcal{D}\left(\mathcal{X}_{r} \times{ }_{\mathbf{P}_{r}} \mathcal{Y}_{r}\right)$ as a kernel on $\mathcal{X}_{r} \times \mathcal{Y}_{r}$. It gives the following kernel functors $\Phi_{r}=\Phi_{\mathcal{E}_{r}}: \mathcal{D}^{b}\left(\mathcal{Y}_{r}\right) \rightarrow \mathcal{D}^{b}\left(\mathcal{X}_{r}\right)$ and $\Phi_{r}^{!}=\Phi_{\mathcal{E}_{r}}: \mathcal{D}^{b}\left(\mathcal{X}_{r}\right) \rightarrow \mathcal{D}^{b}\left(\mathcal{Y}_{r}\right)$. The goal of this section is to show that the functor $\Phi_{r}$ is fully faithful for $r \leq i$, the functor $\Phi_{r}^{!}$is fully faithful for $r \geq i$ and that they give the following semiorthogonal decompositions

$$
\begin{aligned}
& \mathcal{D}^{b}\left(\mathcal{X}_{r}\right)=\left\langle\Phi_{r}\left(\mathcal{D}^{b}\left(\mathcal{Y}_{r}\right)\right), E_{1}(1) \otimes \mathcal{D}^{b}\left(\mathbf{P}_{r}\right), \ldots, E_{2}(i-r) \otimes \mathcal{D}^{b}\left(\mathbf{P}_{r}\right)\right\rangle, \\
& \mathcal{D}^{b}\left(\mathcal{Y}_{r}\right)=\left\langle\Phi_{r}^{!}\left(\mathcal{D}^{b}\left(\mathcal{X}_{r}\right)\right), F_{2}^{*}(i-r) \otimes \mathcal{D}^{b}\left(\mathbf{P}_{r}\right), \ldots, F_{1}^{*}(-1) \otimes \mathcal{D}^{b}\left(\mathbf{P}_{r}\right)\right\rangle .
\end{aligned}
$$

After that we deduce from this the main theorem 1.2 using the faithful base change theorem 2.45.

To prove the fullness and faithfulness of the functors we use induction in $r$ (the base is given by theorem 3.1). To compare the universal families $\mathcal{X}_{r-1}, \mathcal{Y}_{r-1}$ and $\mathcal{X}_{r}, \mathcal{Y}_{r}$ we take for a base scheme

$$
\mathbf{S}_{r}=\mathrm{FI}\left(r-1, r ; V^{*}\right) \cap\left(\mathbf{P}_{r-1} \times \mathbf{P}_{r}\right),
$$

where $\mathrm{FI}\left(r-1, r ; V^{*}\right) \subset \operatorname{Gr}\left(r-1, V^{*}\right) \times \operatorname{Gr}\left(r, V^{*}\right)$ is the partial flag variety. The scheme $\mathbf{S}_{r}$ parameterizes flags $L_{r-1} \subset L_{r} \subset V^{*}$ such that $\operatorname{dim} L_{r-1}=r-1, \operatorname{dim} L_{r}=r$ and $\mathbb{P}\left(L_{r}\right) \cap \mathbf{Z}=\emptyset$.

Let $\phi: \mathbf{S}_{r} \rightarrow \mathbf{P}_{r-1}$ and $\psi: \mathbf{S}_{r} \rightarrow \mathbf{P}_{r}$ denote the natural projections. Let $\tilde{\mathcal{L}}_{r-1}=\phi^{*} \mathcal{L}_{r-1}, \tilde{\mathcal{L}}_{r}=\psi^{*} \mathcal{L}_{r}$, $\tilde{\mathcal{L}}_{r-1}^{\perp}=\phi^{*} \mathcal{L}_{r-1}^{\perp}, \tilde{\mathcal{L}}_{r}^{\perp}=\psi^{*} \mathcal{L}_{r}^{\perp}$. Then we have

$$
\tilde{\mathcal{L}}_{r-1} \subset \tilde{\mathcal{L}}_{r} \subset V^{*} \otimes \mathcal{O}_{\mathbf{S}_{r}}, \quad \tilde{\mathcal{L}}_{r}^{\perp} \subset \tilde{\mathcal{L}}_{r-1}^{\perp} \subset V \otimes \mathcal{O}_{\mathbf{S}_{r}},
$$

Denote

Note that

$$
\begin{array}{lll}
\tilde{\mathcal{X}}_{r-1}=\mathcal{X}_{r-1} \times_{\mathbf{P}_{r-1}} \mathbf{S}_{r} \subset X \times \mathbf{S}_{r}, & & \tilde{\mathcal{X}}_{r}=\mathcal{X}_{r} \times_{\mathbf{P}_{r}} \mathbf{S}_{r} \subset X \times \mathbf{S}_{r}, \\
\tilde{\mathcal{Y}}_{r-1}=\mathcal{Y}_{r-1} \times_{\mathbf{P}_{r-1}} \mathbf{S}_{r} \subset Y \times \mathbf{S}_{r}, & & \tilde{\mathcal{Y}}_{r}=\mathcal{Y}_{r} \times_{\mathbf{P}_{r}} \mathbf{S}_{r} \subset Y \times \mathbf{S}_{r} .
\end{array}
$$

$$
\begin{array}{lll}
\tilde{\mathcal{X}}_{r-1}=\left(X \times \mathbf{S}_{r}\right) \times_{\mathbb{P}(V) \times \mathbf{S}_{r}} \mathbb{P}_{\mathbf{S}_{r}}\left(\tilde{\mathcal{L}}_{r-1}^{\perp}\right), & \tilde{\mathcal{X}}_{r}=\left(X \times \mathbf{S}_{r}\right) \times_{\mathbb{P}(V) \times \mathbf{S}_{r}} \mathbb{P}_{\mathbf{S}_{r}}\left(\tilde{\mathcal{L}}_{r}^{\perp}\right), \\
\tilde{\mathcal{Y}}_{r-1}=\left(Y \times \mathbf{S}_{r}\right) \times_{\mathbb{P}\left(V^{*}\right) \times \mathbf{S}_{r}} \mathbb{P}_{\mathbf{S}_{r}}\left(\tilde{\mathcal{L}}_{r-1}\right), & \tilde{\mathcal{Y}}_{r}=\left(Y \times \mathbf{S}_{r}\right) \times_{\mathbb{P}\left(V^{*}\right) \times \mathbf{S}_{r}} \mathbb{P}_{\mathbf{S}_{r}}\left(\tilde{\mathcal{L}}_{r}\right) .
\end{array}
$$

Therefore the embeddings (20) induce embeddings $\xi: \tilde{\mathcal{X}}_{r} \rightarrow \tilde{\mathcal{X}}_{r-1}$ and $\eta: \tilde{\mathcal{Y}}_{r-1} \rightarrow \tilde{\mathcal{Y}}_{r}$. Consider the following commutative diagrams (the squares marked with EC are exact cartesian, because the maps $\phi$ and $\psi$ are flat)
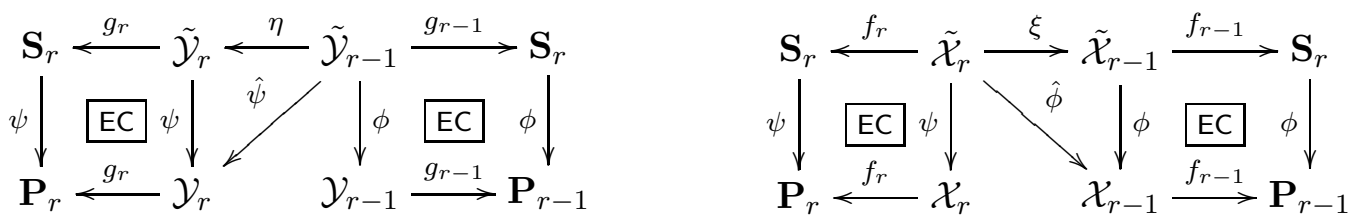

where $f_{r-1}, f_{r}, g_{r-1}$ and $g_{r}$ are the natural projections and $\hat{\phi}=\phi \circ \xi, \hat{\psi}=\psi \circ \eta$.

Let $\tilde{\mathcal{E}}_{r-1} \in \mathcal{D}\left(\tilde{\mathcal{X}}_{r-1} \times_{\mathbf{S}_{r}} \tilde{\mathcal{Y}}_{r-1}\right)$ and $\tilde{\mathcal{E}}_{r} \in \mathcal{D}\left(\tilde{\mathcal{X}}_{r} \times{ }_{\mathbf{S}_{r}} \tilde{\mathcal{Y}}_{r}\right)$ denote the pullbacks of the objects $\mathcal{E}_{r-1}$ and $\mathcal{E}_{r}$ via the projections $\tilde{\mathcal{X}}_{r-1} \times_{\mathbf{s}_{r}} \tilde{\mathcal{Y}}_{r-1} \rightarrow \mathcal{X}_{r-1} \times_{\mathbf{P}_{r-1}} \mathcal{Y}_{r-1}, \tilde{\mathcal{X}}_{r} \times \mathbf{s}_{r} \tilde{\mathcal{Y}}_{r} \rightarrow \mathcal{X}_{r} \times_{\mathbf{P}_{r}} \mathcal{Y}_{r}$. Then we have the corresponding kernel functors $\tilde{\Phi}_{r-1}, \tilde{\Phi}_{r}$ e.t.c between the derived categories of $\tilde{\mathcal{X}}_{r-1}, \tilde{\mathcal{Y}}_{r-1}, \tilde{\mathcal{X}}_{r}$ and $\tilde{\mathcal{Y}}_{r}$.

The induction step is based on relation of the functors $\Phi_{r-1}, \Phi_{r}, \tilde{\Phi}_{r-1}$ and $\tilde{\Phi}_{r}$ to the base change functors $\psi^{*}, \psi_{*}$ and to the functors of the pushforward and pullback via $\xi$ and $\eta$. The relation to $\psi^{*}$ and $\psi_{*}$ is given by lemma 2.41. The relation to $\xi$ and $\eta$ in a sense is the key point of the proof. We prove that $\xi^{*} \tilde{\Phi}_{r-1} \cong \tilde{\Phi}_{r} \eta_{*}$ and that the "difference" between $\xi_{*} \tilde{\Phi}_{r}$ and $\tilde{\Phi}_{r-1} \eta^{!}$is given by a very simple functor, the kernel of which has a resolution of the form $E_{1} \otimes F_{1} \rightarrow E_{2} \otimes F_{2}$ (up to a twist and a shift) on $\tilde{\mathcal{X}}_{r-1} \times_{\mathbf{S}_{r}} \tilde{\mathcal{Y}}_{r}$.

Other results in this section (e.g. the above semiorthogonal decompositions) are proved by similar arguments using (either ascending or descending) induction in $r$.

The section is organized as follows. We start with some preparations concluding with a description of the relation of the functors $\tilde{\Phi}_{r-1}$ and $\tilde{\Phi}_{r}$ to the pushforward and pullback via $\xi$ and $\eta$. Then we 
use induction in $r$ to prove that $\Phi_{r}$ is fully faithful for $r \leq i$. Then we use a relative Bridgeland's trick 2.50 to establish a semiorthogonal decomposition for $r=i$. Then we use descending induction in $r$ to establish semiorthogonal decompositions for $\mathcal{X}_{r}$ when $r \leq i$. Then we show that the collection $\left(F_{1}(1), F_{2}(1), \ldots, F_{1}(N-i), F_{2}(N-i)\right)$ on $Y$ is an exceptional collection in a certain sense (if $\mathbf{Z}=\emptyset$ then it is exceptional in the usual sense). Then we use induction in $r$ to establish semiorthogonal decompositions for $\mathcal{Y}_{r}$ when $r \geq i$. Finally we deduce theorem 1.2 and show that the set of critical values of the morphism $g: Y \rightarrow \mathbb{P}\left(V^{*}\right) \backslash \mathbf{Z}$ coincides with $X^{\vee} \backslash \mathbf{Z}$.

4.2. Preparations. Recall that $\mathbf{P}_{r} \subset \operatorname{Gr}\left(r, V^{*}\right)$ is an open subset parameterizing the compact linear sections of $X$ and $Y$, and $\mathcal{X}_{r}, \mathcal{Y}_{r}$ are the universal families over $\mathbf{P}_{r}$ of linear sections.

Lemma 4.1. Assume that $\mathbf{P}_{r} \neq \emptyset$. Then $\mathcal{X}_{r}$ and $\mathcal{Y}_{r}$ are smooth,

$$
\begin{aligned}
& \operatorname{dim} \mathcal{X}_{r}=\operatorname{dim} X+\operatorname{dim} \mathbf{P}_{r}-r, \quad \operatorname{dim} \mathcal{Y}_{r}=\operatorname{dim} Y+\operatorname{dim} \mathbf{P}_{r}+r-N, \\
& \operatorname{dim} \mathcal{X}_{r} \times_{\mathbf{P}_{r}} \mathcal{Y}_{r}=\operatorname{dim} X+\operatorname{dim} Y+\operatorname{dim} \mathbf{P}_{r}-N,
\end{aligned}
$$

and the maps $f_{r}: \mathcal{X}_{r} \rightarrow \mathbf{P}_{r}$ and $g_{r}: \mathcal{Y}_{r} \rightarrow \mathbf{P}_{r}$ are projective.

Proof: Note that we have open embeddings into the relative Grassmannians

$$
\mathcal{X}_{r} \subset \mathrm{Gr}_{X}\left(r, \mathcal{V}_{X}\right), \quad \mathcal{Y}_{r} \subset \mathrm{Gr}_{Y}\left(r-1, \mathcal{V}_{Y}\right), \quad \mathcal{X}_{r} \times_{\mathbf{P}_{r}} \mathcal{Y}_{r} \subset \mathrm{Gr}_{Q(X, Y)}\left(r-1, \mathcal{V}_{Q}\right),
$$

where the bundles $\mathcal{V}_{X}, \mathcal{V}_{Y}$ are defined from exact sequences

$$
0 \rightarrow \mathcal{V}_{X} \rightarrow V^{*} \otimes \mathcal{O}_{X} \rightarrow \mathcal{O}_{X}(1) \rightarrow 0, \quad 0 \rightarrow \mathcal{O}_{Y}(-1) \rightarrow V^{*} \otimes \mathcal{O}_{Y} \rightarrow \mathcal{V}_{Y} \rightarrow 0
$$

and $\mathcal{V}_{Q}$ is the middle cohomology bundle of the complex

$$
\mathcal{O}_{Q(X, Y)}(0,-1) \rightarrow V^{*} \otimes \mathcal{O}_{Q(X, Y)} \rightarrow \mathcal{O}_{Q(X, Y)}(1,0) .
$$

From this we easily deduce the smoothness and compute the dimensions. It is also clear that the fibers of the projections $\mathcal{X}_{r} \rightarrow \mathbf{P}_{r}$, and $\mathcal{Y}_{r} \rightarrow \mathbf{P}_{r}$ are linear sections of $X$ and $Y$ corresponding to subspaces $L \in \mathbf{P}_{r}$, so they are projective.

Recall that we have defined the objects $\mathcal{E}_{r}$ on $\mathcal{X}_{r} \times_{\mathbf{P}_{r}} \mathcal{Y}_{r}$ as the pullbacks of $\mathcal{E} \in \mathcal{D}^{b}(Q(X, Y))$ via the $\operatorname{map} \zeta_{r}: \mathcal{X}_{r} \times_{\mathbf{P}_{r}} \mathcal{Y}_{r} \rightarrow Q(X, Y)$, and the objects $\tilde{\mathcal{E}}_{r-1}$ and $\tilde{\mathcal{E}}_{r}$ as the pullbacks of $\mathcal{E}_{r-1}$ and $\mathcal{E}_{r}$ via the maps $\phi: \tilde{\mathcal{X}}_{r-1} \times \mathbf{S}_{r} \tilde{\mathcal{Y}}_{r-1} \rightarrow \mathcal{X}_{r-1} \times{ }_{\mathbf{P}_{r-1}} \mathcal{Y}_{r-1}$ and $\psi: \tilde{\mathcal{X}}_{r} \times_{\mathbf{s}_{r}} \tilde{\mathcal{Y}}_{r} \rightarrow \mathcal{X}_{r} \times_{\mathbf{P}_{r}} \mathcal{Y}_{r}$. The functors $\Phi_{r-1}, \Phi_{r}, \Phi_{r-1}^{!}, \Phi_{r}^{!}$, $\tilde{\Phi}_{r-1}, \tilde{\Phi}_{r}, \tilde{\Phi}_{r-1}^{!}$, and $\tilde{\Phi}_{r}^{!}$are the kernel functors of the first and second type corresponding to the kernels $\mathcal{E}_{r-1}, \mathcal{E}_{r}, \tilde{\mathcal{E}}_{r-1}$ and $\tilde{\mathcal{E}}_{r}$ respectively.

Lemma 4.2. The functors $\Phi_{r-1}, \Phi_{r}, \Phi_{r-1}^{!}, \Phi_{r}^{!}, \tilde{\Phi}_{r-1}, \tilde{\Phi}_{r}, \tilde{\Phi}_{r-1}^{!}$, and $\tilde{\Phi}_{r}^{!}$, take the bounded derived categories to the bounded derived categories. Moreover, the functors with the shriek are right adjoint to the corresponding functors without the shriek.

Proof: Since $\mathcal{X}_{r}$ and $\mathcal{Y}_{r}$ are smooth it follows that the pushforward of $\mathcal{E}_{r}$ to $\mathcal{X}_{r} \times \mathcal{Y}_{r}$ is a perfect complex. Therefore, $\mathcal{E}_{r}$ has finite Tor and Ext-amplitude over $\mathcal{X}_{r}$ and $\mathcal{Y}_{r}$ by lemma D.40. Similarly, $\mathcal{E}_{r-1}$ has finite Tor and Ext-amplitude over $\mathcal{X}_{r-1}$ and $\mathcal{Y}_{r-1}$. Since $\phi$ and $\psi$ are flat it follows from corollary D.46 that $\tilde{\mathcal{E}}_{r-1}$ and $\tilde{\mathcal{E}}_{r}$ also have finite Tor and Ext-amplitude. On the other hand, the projections of $\mathcal{X}_{r-1} \times{ }_{\mathbf{P}_{r-1}} \mathcal{Y}_{r-1}$, $\mathcal{X}_{r} \times \mathbf{P}_{r} \mathcal{Y}_{r}, \tilde{\mathcal{X}}_{r-1} \times \mathbf{s}_{r} \tilde{\mathcal{Y}}_{r-1}$ and $\tilde{\mathcal{X}}_{r} \times \mathbf{s}_{r} \tilde{\mathcal{Y}}_{r}$ to the factors are projective because the projections of $\mathcal{X}_{r-1}$, $\mathcal{Y}_{r-1}$ to $\mathbf{P}_{r-1}$ and of $\mathcal{X}_{r}, \mathcal{Y}_{r}$ to $\mathbf{P}_{r}$ are. It remains to apply lemma 2.4.

Lemma 4.3. Let $k=r-1$ or $k=r$. We have

(i) $\tilde{\mathcal{X}}_{k}$ is the zero locus of a section of vector bundle $\mathcal{O}_{X}(1) \otimes \tilde{\mathcal{L}}_{k}^{*}$ on $X \times \mathbf{S}_{r}$;

(ii) $\tilde{\mathcal{Y}}_{k}$ is the zero locus of a section of vector bundle $\mathcal{O}_{Y}(1) \otimes \tilde{\mathcal{L}}_{l}^{\perp *}$ on $Y \times \mathbf{S}_{r}$;

(iii) $\tilde{\mathcal{X}}_{r}$ is the zero locus of a section of line bundle $\mathcal{O}_{X}(1) \otimes\left(\tilde{\mathcal{L}}_{r} / \tilde{\mathcal{L}}_{r-1}\right)^{*}$ on $\tilde{\mathcal{X}}_{r-1}$; 
(iv) $\tilde{\mathcal{Y}}_{r-1}$ is the zero locus of a section of line bundle $\mathcal{O}_{Y}(1) \otimes\left(\tilde{\mathcal{L}}_{r-1}^{\perp} / \tilde{\mathcal{L}}_{r}^{\perp}\right)^{*} \cong \mathcal{O}_{Y}(1) \otimes\left(\tilde{\mathcal{L}}_{r} / \tilde{\mathcal{L}}_{r-1}\right)$ on $\tilde{\mathcal{Y}}_{r}$. All these sections are regular.

Proof: The parts $(i)$ and (ii) evidently follow from the definition of $\tilde{\mathcal{X}}_{k} \subset X \times \mathbf{S}_{r}$. The parts (iii) and (iv) follow from the exact sequences

$$
0 \rightarrow\left(\tilde{\mathcal{L}}_{r} / \tilde{\mathcal{L}}_{r-1}\right)^{*} \rightarrow \tilde{\mathcal{L}}_{r}^{*} \rightarrow \tilde{\mathcal{L}}_{r-1}^{*} \rightarrow 0, \quad 0 \rightarrow\left(\tilde{\mathcal{L}}_{r-1}^{\perp} / \tilde{\mathcal{L}}_{r}^{\perp}\right)^{*} \rightarrow\left(\tilde{\mathcal{L}}_{r-1}^{\perp}\right)^{*} \rightarrow\left(\tilde{\mathcal{L}}_{r}^{\perp}\right)^{*} \rightarrow 0 .
$$

Finally, it follows from lemma 4.1 that $\operatorname{dim} \tilde{\mathcal{X}}_{r-1}=\operatorname{dim} X+\operatorname{dim} \mathbf{S}_{r}-(r-1), \operatorname{dim} \tilde{\mathcal{X}}_{r}=\operatorname{dim} X+\operatorname{dim} \mathbf{S}_{r}-r$. Therefore the sections in the parts $(i)$ and (iii) are regular. The sections in the parts $(i i)$ and $(i v)$ are regular by similar reasons.

Now we describe the maps $\psi$ and $\hat{\psi}$.

Lemma 4.4. The maps $\psi$ and $\hat{\psi}$ are projectivizations of vector bundles. Explicitly, $\psi$ is the projectivization of $\mathcal{L}_{r}^{*}$ and $\hat{\psi}$ is the projectivization of $\left(\mathcal{L}_{r} / \mathcal{O}_{\mathbb{P}\left(V^{*}\right)}(-1)\right)^{*}$, where the embedding $\mathcal{O}_{\mathbb{P}\left(V^{*}\right)}(-1) \rightarrow \mathcal{L}_{r}$ is induced by the projection $\mathcal{Y}_{r} \rightarrow \mathbb{P}_{\mathbf{P}_{r}}\left(\mathcal{L}_{r}\right) \rightarrow \mathbb{P}\left(V^{*}\right)$.

Proof: By definition of $\mathbf{S}_{r}$ the fiber of $\psi$ is the set of all hyperplanes in $L_{r}$. Similarly, the fiber of $\hat{\psi}$ is the set of all hyperplanes in $L_{r}$ passing through a point $y \in \mathbb{P}\left(L_{r}\right)$.

Applying results of $[\mathrm{O} 2]$ we deduce the following.

Corollary 4.5. The functors $\psi^{*}$ and $\hat{\psi}^{*}$ are fully faithful and we have $\psi_{*} \psi^{*} \cong \mathrm{id}, \hat{\psi}_{*} \hat{\psi}^{*} \cong \mathrm{id}$.

The situation with morphisms $\phi$ and $\hat{\phi}$ is a little bit more involved because of the condition $L \cap \mathbf{Z}=\emptyset$ entering in the definition of $\mathbf{P}_{r}$. In fact, it is easy to see that both maps factor as a composition of an open embedding followed by the projectivization of a vector bundle. More precisely, we have

Lemma 4.6. The map $\phi$ is a composition of an open embedding with the projectivization of the vector bundle $V^{*} / \mathcal{L}_{r-1}$. Moreover, any divisorial component of the complement $\mathbb{P}_{\mathbf{P}_{r-1}}\left(V^{*} / \mathcal{L}_{r-1}\right) \backslash \mathbf{S}_{r}$ is ample over $\mathbf{P}_{r-1}$. Similarly, the map $\hat{\phi}$ is a composition of an open embedding with the projectivization of the vector bundle $\mathcal{V}_{X} / \mathcal{L}_{r-1}$, where $\mathcal{V}_{X}$ was defined in lemma 4.1. Moreover, the map $\hat{\phi}$ is surjective if $r \leq i$.

Proof: By definition of $\mathbf{S}_{r}$ the fiber of $\phi$ is the set of all $L_{r}$ containing the given $L_{r-1}$ and nonintersecting with $\mathbf{Z}$. Thus it is an open subset of $\mathbb{P}\left(V^{*} / L_{r-1}\right)$. Moreover, the complement coincides with the preimage of the set of all $L_{r} \in \operatorname{Gr}\left(r, V^{*}\right)$ such that $\mathbb{P}\left(L_{r}\right) \cap \mathbf{Z} \neq \emptyset$ and it remains to note that any effective divisor in $\operatorname{Gr}\left(r, V^{*}\right)$ is ample.

Similarly, the fiber of $\hat{\phi}$ is the set of all $L_{r}$ containing the given $L_{r-1}$, contained in the fiber $\mathcal{V}_{x}$ of the bundle $\mathcal{V}_{X}$ at the given point $x$ and nonintersecting with $\mathbf{Z}$. Thus it is an open subset of $\mathbb{P}\left(\mathcal{V}_{X} / L_{r-1}\right)$. It remains to check the surjectivity. Assume that a point $\left(x, L_{r-1}\right) \in \mathcal{X}_{r-1}$ doesn't lie in the image of $\tilde{\mathcal{X}}_{r}$. This means that for any subspace $L_{r} \subset V^{*}$ such that $L_{r-1} \subset L_{r} \subset \mathcal{V}_{x}$ we have $\mathbb{P}\left(L_{r}\right) \cap \mathbf{Z} \neq \emptyset$. Therefore the linear projection of $\mathbf{Z} \cap \mathbb{P}\left(\mathcal{V}_{x}\right) \subset \mathbb{P}\left(\mathcal{V}_{x}\right) \backslash \mathbb{P}\left(L_{r-1}\right)$ to $\mathbb{P}\left(\mathcal{V}_{x} / L_{r-1}\right)$ is surjective. But then we have $\operatorname{dim}\left(\mathbf{Z} \cap \mathbb{P}\left(\mathcal{V}_{x}\right)\right) \geq \operatorname{dim}\left(\mathbb{P}\left(\mathcal{V}_{x} / L_{r-1}\right)\right)=(N-1)-(r-1)-1=N-r-1 \geq N-i-1$ contradicting (C.8) since $\mathcal{V}_{x}$ is a hyperplane in $V^{*}$.

The functors $\phi^{*}$ and $\hat{\phi}^{*}$ are not fully faithful. However, they enjoy the following properties

Corollary 4.7. If $\phi^{*} \mathcal{L} \cong \mathcal{O}_{\tilde{\mathcal{Y}}_{r-1}}$ for some line bundle $\mathcal{L}$ on $\mathcal{Y}_{r-1}$ and $r \leq i$ then $\mathcal{L} \cong \mathcal{O}_{\mathcal{Y}_{r-1}}$. If $\hat{\phi}^{*} F=0$ for some $F \in \mathcal{D}\left(\mathcal{X}_{r-1}\right)$ and $r \leq i$ then $F=0$.

Proof: For the first claim note that the kernel of the map $\operatorname{Pic}\left(\mathbb{P}_{\mathcal{Y}_{r-1}}\left(V^{*} / \mathcal{L}_{r-1}\right)\right) \rightarrow \operatorname{Pic}\left(\tilde{\mathcal{Y}}_{r-1}\right)$ is generated by divisorial components of the complement $\mathbb{P}_{\mathcal{Y}_{r-1}}\left(V^{*} / \mathcal{L}_{r-1}\right) \backslash \tilde{\mathcal{Y}}_{r-1}$, which are ample over $\tilde{\mathcal{Y}}_{r-1}$. Therefore, this kernel intersects the image of $\operatorname{Pic}\left(\mathcal{Y}_{r-1}\right)$ in $\operatorname{Pic}\left(\mathbb{P}_{\mathcal{Y}_{r-1}}\left(V^{*} / \mathcal{L}_{r-1}\right)\right)$ only at zero. The second claim is evident. 
Now we go to the relation of the functors $\tilde{\Phi}_{r-1}$ and $\tilde{\Phi}_{r}$ to the pushforward and pullback via $\xi$ and $\eta$. Consider the following diagram

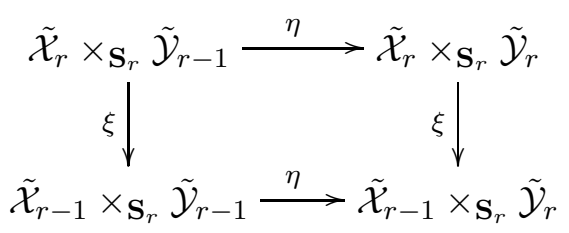

and the projection $\tilde{\pi}: \tilde{\mathcal{X}}_{r-1} \times \mathbf{S}_{r} \tilde{\mathcal{Y}}_{r} \subset\left(X \times \mathbf{S}_{r}\right) \times\left(Y \times \mathbf{S}_{r}\right) \rightarrow X \times Y$.

Lemma 4.8. The maps $\xi$ and $\eta$ in the above diagram are divisorial embeddings, we have the following scheme-theoretical equalities

$$
\begin{aligned}
& \left(\tilde{\mathcal{X}}_{r-1} \times \mathbf{S}_{r} \tilde{\mathcal{Y}}_{r-1}\right) \cap\left(\tilde{\mathcal{X}}_{r} \times \mathbf{S}_{r} \tilde{\mathcal{Y}}_{r}\right)=\tilde{\mathcal{X}}_{r} \times \mathbf{S}_{r} \tilde{\mathcal{Y}}_{r-1}, \\
& \left(\tilde{\mathcal{X}}_{r-1} \times \mathbf{S}_{r} \tilde{\mathcal{Y}}_{r-1}\right) \cup\left(\tilde{\mathcal{X}}_{r} \times \mathbf{S}_{r} \tilde{\mathcal{Y}}_{r}\right)=\tilde{\pi}^{-1}(Q(X, Y)) .
\end{aligned}
$$

and the following square is exact cartesian

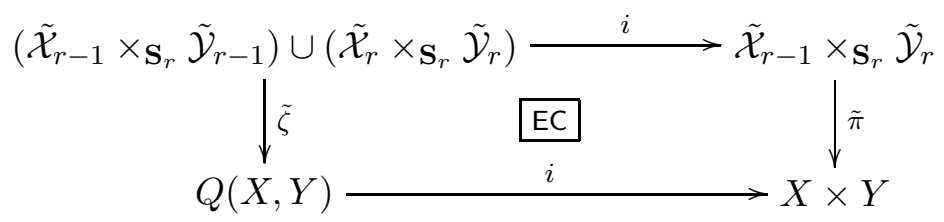

Proof: Consider the projections of $\tilde{\mathcal{X}}_{r} \times \mathbf{s}_{r} \tilde{\mathcal{Y}}_{r-1}, \tilde{\mathcal{X}}_{r-1} \times \mathbf{s}_{r} \tilde{\mathcal{Y}}_{r-1}, \tilde{\mathcal{X}}_{r} \times \mathbf{s}_{r} \tilde{\mathcal{Y}}_{r}$ and $\tilde{\mathcal{X}}_{r-1} \times \mathbf{s}_{r} \tilde{\mathcal{Y}}_{r}$ to $X \times Y$. It is easy to check that their fibers over a point $(x, y) \in(X, Y)$ are open subsets in the subsets of the flag variety $\mathrm{FI}\left(r-1, r ; V^{*}\right)$ consisting of all flags $L_{r-1} \subset L_{r}$ satisfying the following incidence conditions

$$
\begin{aligned}
& y \subset L_{r-1} \quad y \subset L_{r-1} \subset \mathcal{V}_{x} \quad L_{r-1} \quad L_{r-1} \subset \mathcal{V}_{x}
\end{aligned}
$$

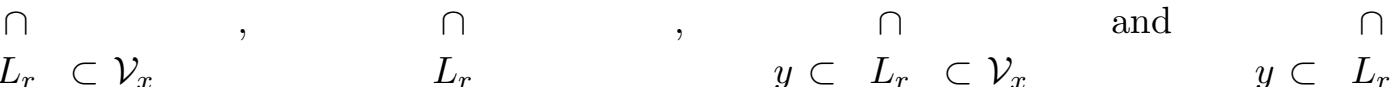

respectively. In particular, the first three fibers are empty if $(x, y) \notin Q(X, Y)$. On the other hand, over $Q(X, Y)$ the first three fibers are irreducible, have dimension $(r-1)(N-r)-1,(r-1)(N-r)$ and $(r-1)(N-r)$ respectively, and the first of them is the intersection of the other two. On the contrary, the fourth fiber is irreducible and $(r-1)(N-r)$-dimensional if $(x, y) \notin Q(X, Y)$ and for $(x, y) \in Q(X, Y)$ it coincides with the union of the second and the third fibers (if $y \subset \mathcal{V}_{x}$ and $y \not \subset L_{r-1}$ then $L_{r}=\left\langle y, L_{r-1}\right\rangle \subset \mathcal{V}_{x}$ ). It follows that images of $\xi$ and $\eta$ have pure codimension 1. Since they are also zero loci of line bundles by lemma 4.3 (iii) and $(i v)$, we conclude that $\xi$ and $\eta$ are divisorial embeddings.

The above arguments also prove the first equality of (22) on the scheme-theoretical level and the second equality on the set-theoretical level. Taking into account that the LHS of the second equality is the zero locus of the line bundle $\mathcal{O}_{X}(1) \otimes \mathcal{O}_{Y}(1)$ by definition of $Q(X, Y)$, and that the RHS of the equality is the zero locus of the line bundle $\left(\mathcal{O}_{X}(1) \otimes\left(\tilde{\mathcal{L}}_{r} / \tilde{\mathcal{L}}_{r-1}\right)^{*}\right) \otimes\left(\mathcal{O}_{Y}(1) \otimes\left(\tilde{\mathcal{L}}_{r} / \tilde{\mathcal{L}}_{r-1}\right)\right)$ by lemma 4.3 (iii) and $(i v)$, and noting that the bundles are isomorphic, we deduce that the second equality is also true on the scheme-theoretical level. Finally, we note that the square (23) is exact cartesian by lemma 2.27 since $X \times Y$ and $Q(X, Y)$ are Cohen-Macaulay.

Consider the pullback $\widehat{\mathcal{E}}=\tilde{\zeta}^{*} \mathcal{E}$ of $\mathcal{E}$ from $Q(X, Y)$ to $\left(\tilde{\mathcal{X}}_{r-1} \times_{\mathbf{S}_{r}} \tilde{\mathcal{Y}}_{r-1}\right) \cup\left(\tilde{\mathcal{X}}_{r} \times_{\mathbf{S}_{r}} \tilde{\mathcal{Y}}_{r}\right)$. Let us also denote $\mathcal{O}(k, l):=\mathcal{O}_{X}(k) \otimes \mathcal{O}_{Y}(l)$ for brevity. The following lemma gives a relation of $\tilde{\mathcal{E}}_{r-1}$ and $\tilde{\mathcal{E}}_{r}$.

Lemma 4.9. We have the following exact sequences on $\tilde{\mathcal{X}}_{r-1} \times \mathbf{S}_{r} \tilde{\mathcal{Y}}_{r}$ :

$$
0 \rightarrow E_{1} \otimes F_{1} \rightarrow E_{2} \otimes F_{2} \rightarrow i_{*} \widehat{\mathcal{E}} \rightarrow 0
$$




$$
\begin{aligned}
& 0 \rightarrow \eta_{*} \tilde{\mathcal{E}}_{r-1}(-1,0) \otimes\left(\tilde{\mathcal{L}}_{r} / \tilde{\mathcal{L}}_{r-1}\right) \rightarrow i_{*} \widehat{\mathcal{E}} \rightarrow \xi_{*} \tilde{\mathcal{E}}_{r} \rightarrow 0, \\
& 0 \rightarrow \xi_{*} \tilde{\mathcal{E}}_{r}(0,-1) \otimes\left(\tilde{\mathcal{L}}_{r} / \tilde{\mathcal{L}}_{r-1}\right)^{*} \rightarrow i_{*} \widehat{\mathcal{E}} \rightarrow \eta_{*} \tilde{\mathcal{E}}_{r-1} \rightarrow 0 .
\end{aligned}
$$

Moreover, we have an isomorphism on $\tilde{\mathcal{X}}_{r} \times \mathbf{s}_{r} \tilde{\mathcal{Y}}_{r-1}$ :

$$
\eta^{*} \tilde{\mathcal{E}}_{r} \cong \xi^{*} \tilde{\mathcal{E}}_{r-1}
$$

Proof: Since the square (23) is exact cartesian we have $i_{*} \widehat{\mathcal{E}}=i_{*} \tilde{\zeta}^{*} \mathcal{E}=\tilde{\pi}^{*} i_{*} \mathcal{E}$ and applying the functor $\tilde{\pi}^{*}$ to $(2)$ we deduce (24). Sequences (25) and (26) can be obtained by tensoring resolutions

$$
\begin{aligned}
& \left.0 \rightarrow \mathcal{O}_{\tilde{\mathcal{X}}_{r-1} \times \mathbf{s}_{r}} \tilde{\mathcal{Y}}_{r-1}(-1,0) \otimes\left(\tilde{\mathcal{L}}_{r} / \tilde{\mathcal{L}}_{r-1}\right) \rightarrow \mathcal{O}_{\left(\tilde{\mathcal{X}}_{r-1} \times \mathbf{s}_{r}\right.} \tilde{\mathcal{Y}}_{r-1}\right) \cup\left(\tilde{\mathcal{X}}_{r} \times_{\mathbf{s}_{r}} \tilde{\mathcal{Y}}_{r}\right) \rightarrow \mathcal{O}_{\tilde{\mathcal{X}}_{r} \times \mathbf{s}_{r}} \tilde{\mathcal{Y}}_{r} \rightarrow 0, \\
& 0 \rightarrow \mathcal{O}_{\tilde{\mathcal{X}}_{r} \times \mathbf{s}_{r}} \tilde{\mathcal{Y}}_{r}(0,-1) \otimes\left(\tilde{\mathcal{L}}_{r} / \tilde{\mathcal{L}}_{r-1}\right)^{*} \rightarrow \mathcal{O}_{\left(\tilde{\mathcal{X}}_{r-1} \times \mathbf{s}_{r} \tilde{\mathcal{Y}}_{r-1}\right) \cup\left(\tilde{\mathcal{X}}_{r} \times \mathbf{s}_{r} \tilde{\mathcal{Y}}_{r}\right)} \rightarrow \mathcal{O}_{\tilde{\mathcal{X}}_{r-1} \times \mathbf{s}_{r}} \tilde{\mathcal{Y}}_{r-1} \rightarrow 0
\end{aligned}
$$

with $\widehat{\mathcal{E}}$ and applying $i_{*}$, since the pullback of $\widehat{\mathcal{E}}$ to $\tilde{\mathcal{X}}_{r-1} \times_{\mathbf{S}_{r}} \tilde{\mathcal{Y}}_{r-1}$ and $\tilde{\mathcal{X}}_{r} \times{ }_{\mathbf{S}_{r}} \tilde{\mathcal{Y}}_{r}$ coincides with $\tilde{\mathcal{E}}_{r-1}$ and $\tilde{\mathcal{E}}_{r}$ respectively. Finally, (27) is evident, because both sides are isomorphic to the pullback of $\mathcal{E}$.

Corollary 4.10. We have the following exact triangles of functors between $\mathcal{D}^{b}\left(\tilde{\mathcal{X}}_{r-1}\right)$ and $\mathcal{D}^{b}\left(\tilde{\mathcal{Y}}_{r}\right)$ :

$$
\begin{gathered}
\tilde{\Phi}_{r-1} \eta^{!} \rightarrow \xi_{*} \tilde{\Phi}_{r} \rightarrow \Phi_{i_{*} \widehat{\mathcal{E}}(0,1) \otimes\left(\tilde{\mathcal{L}}_{r} / \tilde{\mathcal{L}}_{r-1}\right)} \\
\tilde{\Phi}_{r}^{!} \xi^{*} \rightarrow \eta_{*} \tilde{\Phi}_{r-1}^{!} \rightarrow \Phi_{i_{*} \widehat{\mathcal{E}}(1,0) \otimes\left(\tilde{\mathcal{L}}_{r} / \tilde{\mathcal{L}}_{r-1}\right)^{*}}^{!}
\end{gathered}
$$

and the following canonical isomorphisms of functors between $\mathcal{D}^{b}\left(\tilde{\mathcal{X}}_{r}\right)$ and $\mathcal{D}^{b}\left(\tilde{\mathcal{Y}}_{r-1}\right)$ :

$$
\xi^{*} \tilde{\Phi}_{r-1} \cong \tilde{\Phi}_{r} \eta_{*}, \quad \eta^{!} \tilde{\Phi}_{r}^{!} \cong \tilde{\Phi}_{r-1}^{!} \xi_{*}
$$

Proof: Apply lemma 2.7 to the exact triangles (25) and (26) and lemma 2.3 to the isomorphisms (27).

Lemma 4.11. We have

(i) $\Phi_{i_{*} \widehat{\mathcal{E}}(0,1) \otimes\left(\tilde{\mathcal{L}}_{r} / \tilde{\mathcal{L}}_{r-1}\right)}\left(\left\langle F_{1}^{*}(-1) \otimes \mathcal{D}^{b}\left(\mathbf{S}_{r}\right), F_{2}^{*}(-1) \otimes \mathcal{D}^{b}\left(\mathbf{S}_{r}\right)\right\rangle^{\perp}\right)=0$;

(ii) $\Phi_{i_{*} \widehat{\mathcal{E}}(0,1) \otimes\left(\tilde{\mathcal{L}}_{r} / \tilde{\mathcal{L}}_{r-1}\right)}\left(\mathcal{D}^{b}\left(\tilde{\mathcal{Y}}_{r}\right)\right) \subset\left\langle E_{1} \otimes \mathcal{D}^{b}\left(\mathbf{S}_{r}\right), E_{2} \otimes \mathcal{D}^{b}\left(\mathbf{S}_{r}\right)\right\rangle$;

(ii) $\Phi_{i_{*} \widehat{\mathcal{E}}(1,0) \otimes\left(\tilde{\mathcal{L}}_{r} / \tilde{\mathcal{L}}_{r-1}\right)^{*}}^{!}\left(\left\langle E_{1}(1) \otimes \mathcal{D}^{b}\left(\mathbf{S}_{r}\right), E_{2}(1) \otimes \mathcal{D}^{b}\left(\mathbf{S}_{r}\right)\right\rangle^{\perp}\right)=0$.

Proof: Note that for $k=1$ or $k=2$ we have

$\Phi_{E_{k} \otimes F_{k}(1) \otimes\left(\tilde{\mathcal{L}}_{r} / \tilde{\mathcal{L}}_{r-1}\right)}(G) \cong E_{k} \otimes H^{\bullet}\left(\tilde{\mathcal{Y}}_{r}, F_{k}(1) \otimes G\right) \otimes\left(\tilde{\mathcal{L}}_{r} / \tilde{\mathcal{L}}_{r-1}\right) \cong E_{k} \otimes \operatorname{RHom}\left(F_{k}^{*}(-1), G\right) \otimes\left(\tilde{\mathcal{L}}_{r} / \tilde{\mathcal{L}}_{r-1}\right)$

Tensoring exact triangle $(24)$ by $\mathcal{O}(0,1) \otimes\left(\tilde{\mathcal{L}}_{r} / \tilde{\mathcal{L}}_{r-1}\right)$ we deduce from this claims $(i)$ and $(i i)$. Similarly, we have

$$
\Phi_{E_{k}(1) \otimes F_{k} \otimes\left(\tilde{\mathcal{L}}_{r} / \tilde{\mathcal{L}}_{r-1}\right)^{*}}^{!}(G) \cong \mathrm{RHom}\left(E_{k}(1), G\right) \otimes F_{k}^{*} \otimes\left(\tilde{\mathcal{L}}_{r} / \tilde{\mathcal{L}}_{r-1}\right) \otimes \omega_{\tilde{\mathcal{Y}}_{r}}\left[\operatorname{dim} \tilde{\mathcal{Y}}_{r}\right]
$$

whereof we deduce $($ iii). 
4.3. Semiorthogonal collections for linear sections of $X$. We prove in this subsection that the derived category $\mathcal{D}^{b}\left(\mathcal{X}_{r}\right)$ admits a semiorthogonal collection of the form

$$
\left(\Phi_{r}\left(\mathcal{D}^{b}\left(\mathcal{Y}_{r}\right)\right), E_{1}(1) \otimes \mathcal{D}^{b}\left(\mathbf{P}_{r}\right), E_{2}(1) \otimes \mathcal{D}^{b}\left(\mathbf{P}_{r}\right), \ldots, E_{1}(i-r) \otimes \mathcal{D}^{b}\left(\mathbf{P}_{r}\right), E_{2}(i-r) \otimes \mathcal{D}^{b}\left(\mathbf{P}_{r}\right)\right) .
$$

We prove it by induction in $r$. We start with the following

Lemma 4.12. If $r<i$ then the functors $\mathcal{D}^{b}\left(\mathbf{P}_{r}\right) \rightarrow \mathcal{D}^{b}\left(\mathcal{X}_{r}\right), G \mapsto E_{s}(k) \otimes f_{r}^{*} G$ are fully faithful for all $k$. Moreover, the collections

$$
\begin{aligned}
& \left\langle E_{1}(1) \otimes \mathcal{D}^{b}\left(\mathbf{P}_{r}\right), E_{2}(1) \otimes \mathcal{D}^{b}\left(\mathbf{P}_{r}\right), \ldots, E_{1}(i-r) \otimes \mathcal{D}^{b}\left(\mathbf{P}_{r}\right), E_{2}(i-r) \otimes \mathcal{D}^{b}\left(\mathbf{P}_{r}\right)\right\rangle \quad \text { and } \\
& \left\langle E_{1} \otimes \mathcal{D}^{b}\left(\mathbf{P}_{r}\right), E_{2} \otimes \mathcal{D}^{b}\left(\mathbf{P}_{r}\right), \ldots, E_{1}(i-r-1) \otimes \mathcal{D}^{b}\left(\mathbf{P}_{r}\right), E_{2}(i-r-1) \otimes \mathcal{D}^{b}\left(\mathbf{P}_{r}\right)\right\rangle
\end{aligned}
$$

in $\mathcal{D}^{b}\left(\mathcal{X}_{r}\right)$ are semiorthogonal.

Proof: Take any pair $E_{s}(k), E_{t}(l)$ such that either $0<k-l<i-r$, or $k=l$ and $s \geq t$. We have

$$
\begin{aligned}
\operatorname{Hom}\left(E_{s}(k) \otimes f_{r}^{*} G, E_{t}(l) \otimes f_{r}^{*} G^{\prime}\right) \cong H\left(\mathcal{X}_{r}, E_{s}^{*}(-k) \otimes E_{t}(l) \otimes f_{r}^{*} G^{*} \otimes f_{r}^{*} G^{\prime}\right) \cong \\
\\
\cong H\left(X \times \mathbf{P}_{r},\left(E_{s}^{*} \otimes E_{t}(l-k)\right) \otimes\left(G^{*} \otimes G^{\prime}\right) \otimes i_{*} \mathcal{O}_{\mathcal{X}_{r}}\right) .
\end{aligned}
$$

But $i_{*} \mathcal{O}_{\mathcal{X}_{r}}$ by lemma $4.3(i)$ admits a Koszul resolution $i_{*} \mathcal{O}_{\mathcal{X}_{r}} \cong \Lambda^{\bullet}\left(\mathcal{O}_{X}(-1) \otimes \mathcal{L}_{r}\right)$. Moreover, we have

$$
\begin{aligned}
H\left(X \times \mathbf{P}_{r},\left(E_{s}^{*} \otimes E_{t}\right.\right. & \left.(l-k)) \otimes\left(G^{*} \otimes G^{\prime}\right) \otimes \Lambda^{p}\left(\mathcal{O}_{X}(-1) \otimes \mathcal{L}_{r}\right)\right) \cong \\
& \cong H\left(X \times \mathbf{P}_{r},\left(E_{s}^{*} \otimes E_{t}(l-k-p)\right) \otimes\left(G^{*} \otimes G^{\prime} \otimes \Lambda^{p} \mathcal{L}_{r}\right)\right) \cong \\
& \left.\cong H\left(X, E_{s}^{*} \otimes E_{t}(l-k-p)\right) \otimes H\left(\mathbf{P}_{r}, G^{*} \otimes G^{\prime} \otimes \Lambda^{p} \mathcal{L}_{r}\right)\right) \cong \\
& \cong \operatorname{Hom}_{X}\left(E_{s}, E_{t}(l-k-p)\right) \otimes \operatorname{Hom}_{\mathbf{P}_{r}}\left(G, G^{\prime} \otimes \Lambda^{p} \mathcal{L}_{r}\right) .
\end{aligned}
$$

It follows from condition (C.3) that $\operatorname{Hom}_{X}\left(E_{s}, E_{t}(l-k-p)\right)=0$ for $0 \leq p \leq r$ in all cases with the exception of $k=l, s=t$ and $p=0$, when we have $\operatorname{Hom}_{X}\left(E_{s}, E_{t}(l-k-p)\right)=\mathrm{k}$. Therefore the collections are semiorthogonal and we have $\operatorname{Hom}\left(E_{s}(k) \otimes f_{r}^{*} G, E_{s}(k) \otimes f_{r}^{*} G^{\prime}\right) \cong \operatorname{Hom}_{\mathbf{P}_{r}}\left(G, G^{\prime}\right)$, that is the functor $G \mapsto E_{s}(k) \otimes f_{r}^{*} G$ is fully faithful.

Proposition 4.13. For all $1 \leq r \leq i$ we have

(i) $\Phi_{r}\left(\mathcal{D}^{b}\left(\mathcal{Y}_{r}\right)\right) \subset\left\langle E_{1 r}(1) \otimes \mathcal{D}^{b}\left(\mathbf{P}_{r}\right), \ldots, E_{2 r}(i-r) \otimes \mathcal{D}^{b}\left(\mathbf{P}_{r}\right)\right\rangle^{\perp}$;

(ii) the functor $\Phi_{r}: \mathcal{D}^{b}\left(\mathcal{Y}_{r}\right) \rightarrow \mathcal{D}^{b}\left(\mathcal{X}_{r}\right)$ is fully faithful.

Proof: We use induction in $r$. The base of induction, $r=1$ is given by theorem 3.1 and proposition 3.15.

Assume that $(i)$ and $(i i)$ are true for $r-1$ and consider the first diagram of (21). First of all, note that

$$
\tilde{\Phi}_{r-1}\left(\mathcal{D}^{b}\left(\tilde{\mathcal{Y}}_{r-1}\right)\right) \subset\left\langle E_{1}(1) \otimes \mathcal{D}^{b}\left(\mathbf{S}_{r}\right), \ldots, E_{2}(i-r+1) \otimes \mathcal{D}^{b}\left(\mathbf{S}_{r}\right)\right\rangle^{\perp}
$$

by the induction hypothesis and proposition 2.44. On the other hand,

$\Phi_{i_{*} \widehat{\mathcal{E}}(0,1) \otimes\left(\tilde{\mathcal{L}}_{r} / \tilde{\mathcal{L}}_{r-1}\right)}\left(\mathcal{D}^{b}\left(\tilde{\mathcal{Y}}_{r-1}\right)\right) \subset\left\langle E_{1} \otimes \mathcal{D}^{b}\left(\mathbf{S}_{r}\right), E_{2} \otimes \mathcal{D}^{b}\left(\mathbf{S}_{r}\right)\right\rangle \subset\left\langle E_{1}(1) \otimes \mathcal{D}^{b}\left(\mathbf{S}_{r}\right), \ldots, E_{2}(i-r) \otimes \mathcal{D}^{b}\left(\mathbf{S}_{r}\right)\right\rangle^{\perp}$

by lemma 4.11 and lemma 4.12. Taking into account exact triangle (28) we deduce that

$$
\xi_{*} \tilde{\Phi}_{r}\left(\mathcal{D}^{b}\left(\tilde{\mathcal{Y}}_{r}\right)\right) \subset\left\langle E_{1}(1) \otimes \mathcal{D}^{b}\left(\mathbf{S}_{r}\right), \ldots, E_{2}(i-r) \otimes \mathcal{D}^{b}\left(\mathbf{S}_{r}\right)\right\rangle^{\perp}
$$

hence by adjunction we have

$$
\begin{aligned}
& \tilde{\Phi}_{r}\left(\mathcal{D}^{b}\left(\tilde{\mathcal{Y}}_{r}\right)\right) \subset \xi^{*}\left\langle E_{1}(1) \otimes \mathcal{D}^{b}\left(\mathbf{S}_{r}\right), \ldots, E_{2}(i-r) \otimes \mathcal{D}^{b}\left(\mathbf{S}_{r}\right)\right\rangle^{\perp}= \\
& \quad=\left\langle E_{1}(1) \otimes \mathcal{D}^{b}\left(\mathbf{S}_{r}\right), \ldots, E_{2}(i-r) \otimes \mathcal{D}^{b}\left(\mathbf{S}_{r}\right)\right\rangle^{\perp} \subset \psi^{*}\left\langle E_{1}(1) \otimes \mathcal{D}^{b}\left(\mathbf{P}_{r}\right), \ldots, E_{2}(i-r) \otimes \mathcal{D}^{b}\left(\mathbf{P}_{r}\right)\right\rangle^{\perp} .
\end{aligned}
$$

Again by adjunction we have

$$
\psi_{*} \tilde{\Phi}_{r}\left(\mathcal{D}^{b}\left(\tilde{\mathcal{Y}}_{r}\right)\right) \subset\left\langle E_{1}(1) \otimes \mathcal{D}_{35}^{b}\left(\mathbf{P}_{r}\right), \ldots, E_{2}(i-r) \otimes \mathcal{D}^{b}\left(\mathbf{P}_{r}\right)\right\rangle^{\perp}
$$


By corollary 4.5 we have $\psi_{*} \psi^{*} \cong \mathrm{id}_{\mathcal{Y}_{r}}$, hence applying lemma 2.41 we deduce

$$
\Phi_{r}\left(\mathcal{D}^{b}\left(\mathcal{Y}_{r}\right)\right)=\psi_{*} \psi^{*} \Phi_{r}\left(\mathcal{D}^{b}\left(\mathcal{Y}_{r}\right)\right)=\psi_{*} \tilde{\Phi}_{r} \psi^{*}\left(\mathcal{D}^{b}\left(\mathcal{Y}_{r}\right)\right) \subset \psi_{*} \tilde{\Phi}_{r}\left(\mathcal{D}^{b}\left(\tilde{\mathcal{Y}}_{r}\right)\right),
$$

which combined with the previous inclusion proves $(i)$ for $r$.

To prove (ii) we note that by (30) and 4.11 (iii) we have

$$
\Phi_{i_{*} \widehat{\mathcal{E}}(1,0) \otimes\left(\tilde{\mathcal{L}}_{r} / \tilde{\mathcal{L}}_{r-1}\right)^{*}}^{!} \circ \tilde{\Phi}_{r-1}=0 .
$$

Therefore, (29) implies that $\tilde{\Phi}_{r}^{!} \xi^{*} \tilde{\Phi}_{r-1} \cong \eta_{*} \tilde{\Phi}_{r-1}^{!} \tilde{\Phi}_{r-1}$. Combining this with corollary 4.10 and with the induction hypothesis we obtain

$$
\tilde{\Phi}_{r}^{!} \tilde{\Phi}_{r} \eta_{*} \cong \tilde{\Phi}_{r}^{!} \xi^{*} \tilde{\Phi}_{r-1} \cong \eta_{*} \tilde{\Phi}_{r-1}^{!} \tilde{\Phi}_{r-1} \cong \eta_{*}
$$

Finally, applying corollary 4.5 and lemma 2.41 we deduce

$$
\Phi_{r}^{!} \Phi_{r} \cong \Phi_{r}^{!} \Phi_{r} \hat{\psi}_{*} \hat{\psi}^{*} \cong \Phi_{r}^{!} \Phi_{r} \psi_{*} \eta_{*} \hat{\psi}^{*} \cong \psi_{*} \tilde{\Phi}_{r}^{!} \tilde{\Phi}_{r} \eta_{*} \hat{\psi}^{*} \cong \psi_{*} \eta_{*} \hat{\psi}^{*} \cong \hat{\psi}_{*} \hat{\psi}^{*} \cong \mathrm{id} \mathcal{Y}_{r} .
$$

Thus $\Phi_{r}$ is fully faithful and (ii) is proved for $r$.

From the above results we obtain the following

Corollary 4.14. If $r \leq i$ then the collection

$$
\left\langle\Phi_{r}\left(\mathcal{D}^{b}\left(\mathcal{Y}_{r}\right)\right), E_{1}(1) \otimes \mathcal{D}^{b}\left(\mathbf{P}_{r}\right), E_{2}(1) \otimes \mathcal{D}^{b}\left(\mathbf{P}_{r}\right), \ldots, E_{1}(i-r) \otimes \mathcal{D}^{b}\left(\mathbf{P}_{r}\right), E_{2}(i-r) \otimes \mathcal{D}^{b}\left(\mathbf{P}_{r}\right)\right\rangle
$$

is semiorthogonal in $\mathcal{D}^{b}\left(\mathcal{X}_{r}\right)$.

4.4. Fullness. In this subsection we establish fullness of the semiorthogonal collection (31). First of all we use a relative Bridgeland's trick 2.50 to check that $\Phi_{i}: \mathcal{D}^{b}\left(\mathcal{Y}_{i}\right) \rightarrow \mathcal{D}^{b}\left(\mathcal{X}_{i}\right)$ is an equivalence of categories. Then we use descending induction in $r$ to check fullness for $r \leq i$. We also compute here the canonical class of $Y$.

First of all we need the following

Lemma 4.15. We have $\mathbf{P}_{i} \neq \emptyset$.

Proof: Follows from lemma 4.4 and lemma 4.6 by induction in $r$.

Proposition 4.16. The functor $\Phi_{i}: \mathcal{D}^{b}\left(\mathcal{Y}_{i}\right) \rightarrow \mathcal{D}^{b}\left(\mathcal{X}_{i}\right)$ is an equivalence of categories. Moreover, we have $\operatorname{dim} \mathcal{Y}_{i}-\operatorname{dim} \mathbf{P}_{i}=\operatorname{dim} X-i$ and $\omega_{\mathcal{Y}_{i}} \cong \omega_{\mathbf{P}_{i}} \otimes \operatorname{det} \mathcal{L}_{i}^{*}$.

Proof: Note that $\mathcal{X}_{i}$ is smooth and connected by lemma 4.1, $\Phi_{i}$ is fully faithful by proposition 4.13 (ii), and by adjunction formula and lemma 4.3 (i) we have

$$
\omega_{\mathcal{X}_{i}} \cong\left(\omega_{X \times \mathbf{P}_{i}} \otimes \operatorname{det}\left(\mathcal{O}_{X}(1) \otimes \mathcal{L}_{i}^{*}\right)\right)_{\mid \mathcal{X}_{i}} \cong\left(\left(\mathcal{O}_{X}(-i) \otimes \omega_{\mathbf{P}_{i}}\right) \otimes\left(\mathcal{O}_{X}(i) \otimes \operatorname{det} \mathcal{L}_{i}^{*}\right)\right)_{\mid \mathcal{X}_{i}} \cong \omega_{\mathbf{P}_{i}} \otimes \operatorname{det} \mathcal{L}_{i}^{*},
$$

and $\operatorname{dim} \mathcal{X}_{i}-\operatorname{dim} \mathbf{P}_{i}=\operatorname{dim} X-i$. Note that the line bundle $\omega_{\mathbf{P}_{i}} \otimes \operatorname{det} \mathcal{L}_{i}^{*}$ is a pullback from $\mathbf{P}_{i}$, hence, by proposition 2.50 the functor $\Phi_{i}$ is an equivalence, $\operatorname{dim} \mathcal{Y}_{i}-\operatorname{dim} \mathbf{P}_{i}=\operatorname{dim} X-i$ and $\omega_{\mathcal{Y}_{i}} \cong \omega_{\mathbf{P}_{i}} \otimes \operatorname{det} \mathcal{L}_{i}^{*}$.

Now we compute the relative canonical classes of $\mathcal{Y}_{r}$ over $\mathbf{P}_{r}$ by descending induction in $r$. This allows to compute the canonical class of $Y$.

Lemma 4.17. For all $r \leq i$ we have $\omega_{\mathcal{Y}_{r} / \mathbf{P}_{r}} \cong \operatorname{det} \mathcal{L}_{r}^{*}(i-r)$.

Proof: We use descending induction in $r$. The base of induction, $r=i$, follows from proposition 4.16. Indeed, $\omega_{\mathcal{Y}_{i} / \mathbf{P}_{i}} \cong \omega_{\mathcal{Y}_{i}} \otimes g^{*} \omega_{\mathbf{P}_{i}}^{-1} \cong \operatorname{det} \mathcal{L}_{i}^{*}$. Now assume that the claim is true for $r$ and consider the diagrams (21). Using the adjunction formula and lemma $4.3(i v)$ we deduce

$$
\begin{aligned}
\omega_{\tilde{\mathcal{Y}}_{r-1} / \mathbf{S}_{r}} \cong \omega_{\tilde{\mathcal{Y}}_{r} / \mathbf{S}_{r}} \otimes\left(\tilde{\mathcal{L}}_{r} / \tilde{\mathcal{L}}_{r-1}\right)(1) \cong \operatorname{det} \tilde{\mathcal{L}}_{r}^{*}(i-r) & \otimes\left(\tilde{\mathcal{L}}_{r} / \tilde{\mathcal{L}}_{r-1}\right)(1) \cong \\
& \cong \operatorname{det} \tilde{\mathcal{L}}_{r-1}^{*}(i-(r-1)) \cong \phi^{*} \operatorname{det} \mathcal{L}_{r-1}^{*}(i-(r-1)) .
\end{aligned}
$$


On the other hand, $\omega_{\tilde{\mathcal{Y}}_{r-1} / \mathbf{S}} \cong \phi^{*} \omega_{\mathcal{Y}_{r-1} / \mathbf{P}_{r-1}}$ since $\phi$ is flat. Therefore $\omega_{\mathcal{Y}_{r-1} / \mathbf{P}_{r-1}} \cong \operatorname{det} \mathcal{L}_{r-1}^{*}(i-(r-1))$ by corollary 4.7 .

Corollary 4.18. We have $\omega_{Y} \cong \mathcal{O}_{Y}(i-N)$ and $\operatorname{dim} Y=\operatorname{dim} X+N-2 i$.

Proof: Note that $\mathcal{Y}_{1}=Y, \mathbf{P}_{1}=\mathbf{P}$ and $\operatorname{det} \mathcal{L}_{1} \cong \mathcal{L}_{1} \cong \mathcal{O}_{Y}(-1)$. Hence

$$
\omega_{Y} \cong \omega_{Y / \mathbf{P}} \otimes \omega_{\mathbf{P}} \cong \omega_{\mathcal{Y}_{1} / \mathbf{P}_{1}} \otimes \omega_{\mathbf{P}} \cong\left(\mathcal{O}_{Y}(1) \otimes \mathcal{O}_{Y}(i-1)\right) \otimes \mathcal{O}_{Y}(-N) \cong \mathcal{O}_{Y}(i-N) .
$$

On the other hand, by lemma 4.1 we have $\operatorname{dim} \mathcal{Y}_{i}-\operatorname{dim} \mathbf{P}_{i}=\operatorname{dim} Y+i-N$, hence by proposition 4.16 we have $\operatorname{dim} Y+i-N=\operatorname{dim} X-i$, whereof $\operatorname{dim} Y=\operatorname{dim} X+N-2 i$.

Now we establish semiorthogonal decompositions for the derived categories $\mathcal{D}^{b}\left(\mathcal{X}_{r}\right)$ with $r \leq i$.

Theorem 4.19. For any $r \leq i$ we have a semiorthogonal decomposition

$\mathcal{D}^{b}\left(\mathcal{X}_{r}\right)=\left\langle\Phi_{r}\left(\mathcal{D}^{b}\left(\mathcal{Y}_{r}\right)\right), E_{1}(1) \otimes \mathcal{D}^{b}\left(\mathbf{P}_{r}\right), E_{2}(1) \otimes \mathcal{D}^{b}\left(\mathbf{P}_{r}\right), \ldots, E_{1}(i-r) \otimes \mathcal{D}^{b}\left(\mathbf{P}_{r}\right), E_{2}(i-r) \otimes \mathcal{D}^{b}\left(\mathbf{P}_{r}\right)\right\rangle$

Proof: We use descending induction in $r$. The base of induction, $r=i$, is given by proposition 4.16. Assume that (32) is true for $r$. Then by the faithful base change theorem 2.45 we have

$$
\mathcal{D}^{b}\left(\tilde{\mathcal{X}}_{r}\right)=\left\langle\tilde{\Phi}_{r}\left(\mathcal{D}^{b}\left(\tilde{\mathcal{Y}}_{r}\right)\right), E_{1}(1) \otimes \mathcal{D}^{b}\left(\mathbf{S}_{r}\right), \ldots, E_{2}(i-r) \otimes \mathcal{D}^{b}\left(\mathbf{S}_{r}\right)\right\rangle
$$

Take arbitrary

$$
G \in\left\langle\Phi_{r-1}\left(\mathcal{D}^{b}\left(\mathcal{Y}_{r-1}\right)\right), E_{1}(1) \otimes \mathcal{D}^{b}\left(\mathbf{P}_{r-1}\right), \ldots, E_{2}(i-r+1) \otimes \mathcal{D}^{b}\left(\mathbf{P}_{r-1}\right)\right\rangle^{\perp} .
$$

Consider the diagram (21). By lemma 2.36 we have $f_{r-1 *}$ RHom $\left(E_{s}(k), G\right)=0$ for $s=1,2$ and $1 \leq k \leq$ $i-r+1$. Therefore

$$
f_{r-1_{*}} \operatorname{RHom}\left(\phi^{*} E_{s}(k), \phi^{*} G\right)=f_{r-1_{*}} \phi^{*} \mathrm{RH} \text { om }\left(E_{s}(k), G\right)=\phi^{*} f_{r-1_{*}} \operatorname{RHom}\left(E_{s}(k), G\right)=0
$$

since the right square in (21) is exact cartesian. Therefore

$$
\phi^{*} G \in\left\langle E_{1}(1) \otimes \mathcal{D}^{b}\left(\mathbf{S}_{r}\right), \ldots, E_{2}(i-r+1) \otimes \mathcal{D}^{b}\left(\mathbf{S}_{r}\right)\right\rangle^{\perp}
$$

On the other hand, it follows from lemma 4.3 (iii) that we have a resolution

$$
0 \rightarrow E_{s}(k) \otimes\left(\tilde{\mathcal{L}}_{r} / \tilde{\mathcal{L}}_{r-1}\right) \rightarrow E_{s}(k+1) \rightarrow \xi_{*} E_{s}(k+1) \rightarrow 0,
$$

hence

$$
\phi^{*} G \in\left\langle\xi_{*} E_{1}(2) \otimes \mathcal{D}^{b}\left(\mathbf{S}_{r}\right), \ldots, \xi_{*} E_{2}(i-r+1) \otimes \mathcal{D}^{b}\left(\mathbf{S}_{r}\right)\right\rangle^{\perp}
$$

and by adjunction

$$
\xi^{!} \phi^{*} G \in\left\langle E_{1}(2) \otimes \mathcal{D}^{b}\left(\mathbf{S}_{r}\right), \ldots, E_{2}(i-r+1) \otimes \mathcal{D}^{b}\left(\mathbf{S}_{r}\right)\right\rangle^{\perp} .
$$

Further, $\xi^{!} \phi^{*} G \cong \xi^{*} \phi^{*} G \otimes\left(\tilde{\mathcal{L}}_{r} / \tilde{\mathcal{L}}_{r-1}\right)^{*}(1)[-1] \cong \hat{\phi}^{*} G \otimes\left(\tilde{\mathcal{L}}_{r} / \tilde{\mathcal{L}}_{r-1}\right)^{*}(1)[-1]$, therefore

$$
\hat{\phi}^{*} G \in\left\langle E_{1}(1) \otimes \mathcal{D}^{b}\left(\mathbf{S}_{r}\right), \ldots, E_{2}(i-r) \otimes \mathcal{D}^{b}\left(\mathbf{S}_{r}\right)\right\rangle^{\perp} .
$$

On the other hand, (34) and lemma 4.11 (iii) imply that $\Phi_{i_{*} \widehat{\mathcal{E}}(1,0) \otimes\left(\tilde{\mathcal{L}}_{r} / \tilde{\mathcal{L}}_{r-1}\right)^{*}}\left(\phi^{*} G\right)=0$. Therefore, applying exact triangle $(29)$ to $\phi^{*} G$ we deduce that $\tilde{\Phi}_{r}^{!} \xi^{*} \phi^{*} G \cong \eta_{*} \tilde{\Phi}_{r-1}^{!} \phi^{*} G$. But $\tilde{\Phi}_{r-1}^{!} \phi^{*}(G) \cong \phi^{*} \Phi_{r-1}^{!}(G)=0$, since $G \in \Phi_{r-1}\left(\mathcal{D}^{b}\left(\mathcal{Y}_{r-1}\right)\right)^{\perp}$ by assumption. Thus we deduce that $\tilde{\Phi}_{r}^{!} \hat{\phi}^{*}(G)=0$, hence

$$
\hat{\phi}^{*} G \in \tilde{\Phi}_{r}\left(\mathcal{D}^{b}\left(\tilde{\mathcal{Y}}_{r}\right)\right)^{\perp} .
$$

Combining this with (35) and (33) we deduce that $\hat{\phi}^{*} G=0$. Therefore $G=0$ by corollary 4.7 and we obtain decomposition (32) for $r-1$. 
4.5. Exceptional collection for $Y$. In this subsection we show that in a certain sense the collection $\left(F_{1}(1), F_{2}(1), \ldots, F_{1}(N-i), F_{2}(N-i)\right)$ on $Y$ is exceptional. The idea behind the proof is very simple. It is easy to see that if a collection $(E(1), \ldots, E(i))$ on a Fano variety $X$ of index $i$ is exceptional then the restriction of the bundle $E$ to a Calabi-Yau linear section of $X$ is also exceptional. It turns out that the inverse is also true: if the restrictions of a vector bundle $E$ to all Calabi-Yau linear sections of $X$ are exceptional then the collection $(E(1), \ldots, E(i))$ is exceptional on $X$. We use this idea as follows. First of all we consider an exceptional pair $\left(E_{0}(1), E_{1}(1)\right)$ on $X$ obtained from $\left(E_{1}, E_{2}\right)$ by a mutation and a twist, and show that the functor $\Phi_{i}^{!}$takes this pair to the pair $\left(F_{2}^{*}, F_{1}^{*}\right)$ on $\mathcal{Y}_{i}$ up to a twist and a shift. Since $\Phi_{i}$ is an equivalence, its adjoint functor $\Phi_{i}^{!}$is an equivalence as well, hence we deduce that the pair $\left(F_{2}^{*}, F_{1}^{*}\right)$ is exceptional on $\mathcal{Y}_{i}$. But this pair is formed by the restrictions of a pair $\left(F_{2}^{*}, F_{1}^{*}\right)$ on $Y$ to the Calabi-Yau sections, hence the above argument shows that the collection $\left(F_{2}^{*}(1), F_{1}^{*}(1), \ldots, F_{2}^{*}(N-i), F_{1}^{*}(N-i)\right)$ on $Y$ is exceptional.

Now we give a detailed proof. We define an object $E_{0} \in \mathcal{D}(X)$ as the mutation of $E_{2}$ through $E_{1}$ (see $[\mathrm{B}]$ ). Thus we have the following exact triangle

$$
E_{0} \rightarrow \operatorname{RHom}\left(E_{1}, E_{2}\right) \otimes E_{1} \rightarrow E_{2}
$$

Lemma 4.20 (cf. [B]). We have

$$
\operatorname{RHom}\left(E_{1}, E_{0}(k)\right)=0, \quad \text { if } 1-i \leq k \leq 0, \quad \operatorname{RHom}\left(E_{2}, E_{0}(k)\right)= \begin{cases}0, & \text { if } 1-i \leq k \leq-1, \\ \mathrm{k}[-1], & \text { if } k=0 .\end{cases}
$$

Proof: Apply $\operatorname{RHom}\left(E_{1},-\right)$ and $\operatorname{RHom}\left(E_{2},-\right)$ to (36).

Proposition 4.21. For all $1 \leq r \leq i$ and $s=0,1$ we have

$$
\Phi_{r}^{!}\left(E_{s}(k)\right)= \begin{cases}0, & \text { for } r+1-i \leq k \leq 0 \\ F_{2-s}^{*}(i-r-1) \otimes \operatorname{det} \mathcal{L}_{r}^{*}[\operatorname{dim} Y-N+r], & \text { for } k=1 .\end{cases}
$$

Proof: We use induction in $r$. First, let us check the case $r=1$. Consider the diagram

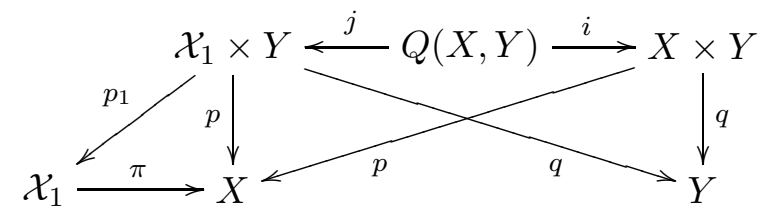

Note that the bundle $E_{s}(k)$ on $\mathcal{X}_{1}$ is a pullback via $\pi$. But $\left.\Phi_{1}^{!}\left(\pi^{*} G\right) \cong \Phi_{1}^{!}\left(\pi^{!} G \otimes \omega_{\mathcal{X}_{1} / X}^{-1}\left[\operatorname{dim} X-\operatorname{dim} \mathcal{X}_{1}\right]\right)\right)$ and since by adjunction formula and lemma $4.3(i) \omega_{\mathcal{X}_{1} / X} \cong \omega_{\mathbf{P}} \otimes\left(\mathcal{O}_{X}(1) \otimes \mathcal{L}_{1}^{*}\right), \operatorname{dim} \mathcal{X}_{1}=\operatorname{dim} X+N-2$, and since the functor $\Phi_{1}^{!}$is $\mathbf{P}$-linear we have

$$
\Phi_{1}^{!}\left(\pi^{*} G\right) \cong \Phi_{1}^{!}\left(\pi^{!}(G(-1)) \otimes \omega_{\mathbf{P}}^{-1} \otimes \mathcal{L}_{1}[2-N] .\right.
$$

On the other hand, it is clear that the functor $\Phi_{1}^{!} \circ \pi^{!}$is a kernel functor of the second type with kernel $\left(\pi \times \mathrm{id}_{Y}\right)_{*} \mathcal{E}_{1} \cong\left(\pi \times \mathrm{id}_{Y}\right)_{*} j_{*} \mathcal{E} \cong i_{*} \mathcal{E}$. Therefore

$$
\Phi_{1}^{!}\left(\pi^{!}(G(-1)) \cong \Phi_{i_{*} \mathcal{E}}(G(-1))=q_{*} \operatorname{RHom}\left(i_{*} \mathcal{E}, p^{!}(G(-1))\right) \cong q_{*} \operatorname{RHom}\left(i_{*} \mathcal{E}, G(-1) \otimes \omega_{Y}[\operatorname{dim} Y]\right) .\right.
$$

Using resolution (2) combined with lemma 4.20 we obtain $\Phi_{1}^{!}\left(\pi^{!}\left(E_{s}(k-1)\right)=0\right.$ for $s=0,1$ and $2-i \leq$ $k \leq 0$ and for $k=1$ we obtain the following exact triangles

$$
\begin{aligned}
& \Phi_{1}^{!}\left(\pi^{!}\left(E_{0}(-1)\right) \rightarrow F_{2}^{*} \otimes \omega_{Y}[\operatorname{dim} Y-1] \rightarrow 0,\right. \\
& \Phi_{1}^{!}\left(\pi^{!}\left(E_{1}(-1)\right) \rightarrow 0 \rightarrow F_{1}^{*} \otimes \omega_{Y}[\operatorname{dim} Y] .\right.
\end{aligned}
$$


Finally, we get

$$
\Phi_{1}^{!}\left(\pi ^ { ! } ( E _ { s } ( k - 1 ) ) \cong \left\{\begin{array}{ll}
0, & \text { for } 2-i \leq k \leq 0, s=0,1 \\
F_{2-s}^{*} \otimes \omega_{Y}[\operatorname{dim} Y-1], & \text { for } k=1, s=0,1
\end{array}\right.\right.
$$

Substituting this into (37) and taking into account the isomorphisms $\omega_{Y} \cong \mathcal{O}_{Y}(i-N)$ (see corollary 4.18), $\omega_{\mathbf{P}}^{-1} \cong \mathcal{O}_{Y}(N)$ and $\operatorname{det} \mathcal{L}_{1} \cong \mathcal{L}_{1} \cong \mathcal{O}_{Y}(-1)$ we deduce the claim for $r=1$.

Now, assume that the claim is true for $r-1$. Consider the diagram (21). We have

$$
\begin{aligned}
\tilde{\Phi}_{r-1}^{!}\left(E_{s}(k)\right) \cong \tilde{\Phi}_{r-1}^{!} \phi^{*}\left(E_{s}(k)\right) \cong \phi^{*} \Phi_{r-1}^{!}\left(E_{s}(k)\right) \cong & \\
& \cong \begin{cases}0, & \text { for } r-i \leq k \leq 0, s=0,1 ; \\
\phi^{*} F_{2-s}^{*}(i-r) \otimes \operatorname{det} \mathcal{L}_{r-1}^{*}[\operatorname{dim} Y-N+r-1], & \text { for } k=1, s=0,1 .\end{cases}
\end{aligned}
$$

by lemma 2.41 and the induction hypothesis. On the other hand,

$$
\begin{aligned}
\hat{\psi}^{*} \Phi_{r}^{!}\left(E_{s}(k)\right) & \cong \eta^{*} \psi^{*} \Phi_{r}^{!}\left(E_{s}(k)\right) \cong \eta^{*} \tilde{\Phi}_{r}^{!}\left(\psi^{*} E_{s}(k)\right) \cong \\
& \cong \eta^{!} \tilde{\Phi}_{r}^{!}\left(E_{s}(k)\right) \otimes \mathcal{O}_{Y}(-1) \otimes\left(\tilde{\mathcal{L}}_{r} / \tilde{\mathcal{L}}_{r-1}\right)^{*}[1] \cong \tilde{\Phi}_{r-1}^{!}\left(\xi_{*} E_{s}(k)\right) \otimes \mathcal{O}_{Y}(-1) \otimes\left(\tilde{\mathcal{L}}_{r} / \tilde{\mathcal{L}}_{r-1}\right)^{*}[1]
\end{aligned}
$$

by lemma 2.41, lemma $4.3(i v)$, and lemma 4.10. Taking into account resolution

$$
0 \rightarrow E_{s}(k-1) \otimes\left(\tilde{\mathcal{L}}_{r} / \tilde{\mathcal{L}}_{r-1}\right) \rightarrow E_{s}(k) \rightarrow \xi_{*} E_{s}(k) \rightarrow 0,
$$

and (38) we deduce

$$
\hat{\psi}^{*} \Phi_{r}^{!}\left(E_{s}(k)\right) \cong \begin{cases}0, & \text { for } r+1-i \leq k \leq 0, s=0,1 \\ F_{2-s}^{*}(i-r-1) \otimes \operatorname{det} \tilde{\mathcal{L}}_{r}^{*}[\operatorname{dim} Y-N+r], & \text { for } k=1, s=0,1 .\end{cases}
$$

Finally, applying corollary 4.5 we deduce the claim.

Corollary 4.22. Let $s, t=1,2$. Then

$$
g_{i *} \operatorname{RHom}_{\mathcal{Y}_{i}}\left(F_{s}, F_{t}\right) \cong \begin{cases}\mathcal{O}_{\mathbf{P}_{i}} \oplus \operatorname{det} \mathcal{L}_{i}[i-\operatorname{dim} X], & \text { for } s=t \\ W \otimes \mathcal{O}_{\mathbf{P}_{i}}, & \text { for } s=1, t=2 \\ W^{*} \otimes \operatorname{det} \mathcal{L}_{i}[i-\operatorname{dim} X], & \text { for } s=2, t=1\end{cases}
$$

Proof: By proposition 4.21, proposition 2.37 and proposition 4.16 we have

$$
\begin{aligned}
& g_{i *} \operatorname{RHom}_{\mathcal{Y}_{i}}\left(F_{s}, F_{t}\right) \cong \\
& \cong g_{i *} \mathrm{RH}_{\mathrm{H}} \mathrm{y}_{\mathcal{Y}_{i}}\left(F_{t}^{*}(-1) \otimes \operatorname{det} \mathcal{L}_{i}^{*}[\operatorname{dim} Y-N+i], F_{s}^{*}(-1) \otimes \operatorname{det} \mathcal{L}_{i}^{*}[\operatorname{dim} Y-N+i]\right) \cong \\
& \cong g_{i *} \operatorname{RH}_{\operatorname{om}_{\mathcal{Y}_{i}}}\left(\Phi_{i}^{!}\left(E_{2-t}(1)\right), \Phi_{i}^{!}\left(E_{2-s}(1)\right)\right) \cong f_{i_{*}} \mathrm{RH} \operatorname{om}_{\mathcal{X}_{i}}\left(\Phi_{i} \Phi_{i}^{!}\left(E_{2-t}(1)\right), E_{2-s}(1)\right) \cong \\
& \cong f_{i_{*}} \mathrm{RH} \operatorname{Hom}_{\mathcal{X}_{i}}\left(E_{2-t}(1), E_{2-s}(1)\right) \cong f_{i *} \mathrm{RH} \operatorname{om}_{\mathcal{X}_{i}}\left(E_{2-t}, E_{2-s}\right) \text {. }
\end{aligned}
$$

On the other hand, $f_{i_{*}} \mathrm{R \mathcal {H }} \mathrm{om}_{\mathcal{X}_{i}}\left(E_{2-t}, E_{2-s}\right) \cong f_{i_{*}}\left(E_{2-t}^{*} \otimes E_{2-s}\right)$. Tensoring the Koszul resolution of $\mathcal{X}_{i}$ in $X \times \mathbf{P}_{i}$ (see lemma $4.3(i)$ ) by $E_{2-t}^{*} \otimes E_{2-s}$ we see that the pushforward of $E_{2-t}^{*} \otimes E_{2-s}$ from $\mathcal{X}_{i}$ to $X \times \mathbf{P}_{i}$ is quasiisomorphic to the complex

$\left(E_{2-t}^{*} \otimes E_{2-s}(-i)\right) \otimes \operatorname{det} \mathcal{L}_{i} \rightarrow\left(E_{2-t}^{*} \otimes E_{2-s}(1-i)\right) \otimes \Lambda^{i-1} \mathcal{L}_{i} \rightarrow \cdots \rightarrow\left(E_{2-t}^{*} \otimes E_{2-s}(-1)\right) \otimes \mathcal{L}_{i} \rightarrow E_{2-t}^{*} \otimes E_{2-s}$

Applying the functor $f_{i_{*}}$ and using the Künneth formula on $X \times \mathbf{P}_{i}$ and (C.3) we deduce the claim. 
Proposition 4.23. If $r \geq i$ and $\mathbf{P}_{r} \neq \emptyset$ then

$$
\mathcal{H}^{p}\left(g _ { r * } \operatorname { R H o m } _ { \mathcal { Y } _ { r } } ( F _ { s } ( k ) , F _ { t } ( l ) ) \cong \left\{\begin{array}{ll}
\mathcal{O}_{\mathbf{P}_{r}}, & \text { if } s=t, k=l \text { and } p=0, \\
\operatorname{det} \mathcal{L}_{r}, & \text { if } s=t, k-l=r-i \\
& \text { and } p=\operatorname{dim} X+r-2 i, \\
W \otimes \mathcal{O}_{\mathbf{P}_{r}}, & \text { if } s=1, t=2, k=l \text { and } p=0, \\
W^{*} \otimes \operatorname{det} \mathcal{L}_{r}, & \text { if } s=2, t=1, k-l=r-i \\
& \text { and } p=\operatorname{dim} X+r-2 i, \\
0, & \text { otherwise, } \text { if } 0 \leq k-l \leq r-i .
\end{array}\right.\right.
$$

Proof: We use induction in $r$. The base of induction, $r=i$, is given by corollary 4.22. Now assume that the claim is true for $r-1$ and consider the diagram (21). Let us denote

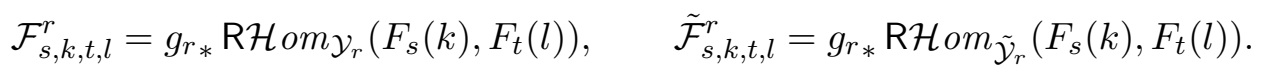

First of all we note that

$$
\begin{aligned}
& \tilde{\mathcal{F}}_{s, k, t, l}^{r-1}=g_{r-1 *} \mathrm{RH}_{\mathrm{H}^{\prime} m_{\tilde{\mathcal{Y}}_{r-1}}}\left(F_{s}(k), F_{t}(l)\right) \cong \\
& \cong g_{r-1_{*}} \phi^{*} \mathrm{R} \mathcal{H} \operatorname{om}_{\mathcal{Y}_{r-1}}\left(F_{s}(k), F_{t}(l)\right) \cong \phi^{*} g_{r-1 *} \mathrm{R} \mathcal{H} \operatorname{om}_{\mathcal{Y}_{r-1}}\left(F_{s}(k), F_{t}(l)\right)=\phi^{*} \mathcal{F}_{s, k, t, l}^{r-1} \text {. }
\end{aligned}
$$

and similarly

$$
\tilde{\mathcal{F}}_{s, k, t, l}^{r} \cong \psi^{*} \mathcal{F}_{s, k, t, l}^{r} .
$$

On the other hand, by $4.3(\mathrm{iv})$ we have a resolution

$$
0 \rightarrow F_{t}(l-1) \otimes\left(\tilde{\mathcal{L}}_{r} / \tilde{\mathcal{L}}_{r-1}\right)^{*} \rightarrow F_{t}(l) \rightarrow \eta_{*} F_{t}(l) \rightarrow 0 .
$$

Applying to it the functor $g_{r *} \mathrm{RH}^{\mathrm{H}} \mathrm{m}_{\tilde{\mathcal{Y}}_{r}}\left(F_{s}(k),-\right)$ and taking into account that

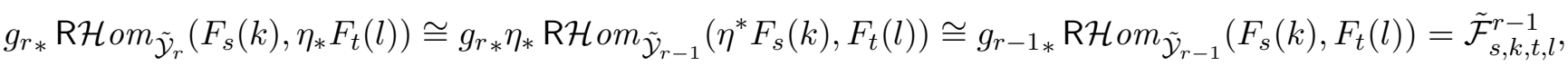
we deduce the following exact triangle on $\mathbf{S}$

$$
\tilde{\mathcal{F}}_{s, k, t, l-1}^{r} \otimes\left(\tilde{\mathcal{L}}_{r} / \tilde{\mathcal{L}}_{r-1}\right)^{*} \rightarrow \tilde{\mathcal{F}}_{s, k, t, l}^{r} \rightarrow \tilde{\mathcal{F}}_{s, k, t, l}^{r-1} .
$$

Since $\tilde{\mathcal{F}}_{s, k, t, l}^{r-1} \cong \phi^{*} \mathcal{F}_{s, k, t, l}^{r-1}, \tilde{\mathcal{F}}_{s, k, t, l}^{r} \cong \psi^{*} \mathcal{F}_{s, k, t, l}^{r}$ we can rewrite this as

$$
\psi^{*}\left(\mathcal{F}_{s, k, t, l-1}^{r}\right) \otimes\left(\tilde{\mathcal{L}}_{r} / \tilde{\mathcal{L}}_{r-1}\right)^{*} \rightarrow \psi^{*}\left(\mathcal{F}_{s, k, t, l}^{r}\right) \rightarrow \phi^{*} \mathcal{F}_{s, k, t, l}^{r-1} .
$$

Finally, note that

$$
\psi_{*}\left(\tilde{\mathcal{L}}_{r} / \tilde{\mathcal{L}}_{r-1}\right)^{\otimes-k}= \begin{cases}\mathcal{O}_{\mathbf{P}_{r}}, & \text { if } k=0, \\ 0, & \text { if } 0 \leq k \leq r-1, \\ \operatorname{det} \mathcal{L}_{r}^{*}[1-r], & \text { if } k=r\end{cases}
$$

by lemma 4.4 (because $\tilde{\mathcal{L}}_{r} / \tilde{\mathcal{L}}_{r-1}$ is the Grothendieck line bundle on $\mathbf{S}_{r} \subset \mathbb{P}_{\mathbf{P}_{r}}\left(\mathcal{L}_{r}^{*}\right)$ ). Therefore, applying the functor $\psi_{*}$ to the triangle (39) we get

$$
\mathcal{F}_{s, k, t, l}^{r} \cong \psi_{*} \phi^{*} \mathcal{F}_{s, k, t, l}^{r-1},
$$

by corollary 4.5 , and applying the functor $\psi_{*}$ to the triangle $(39)$ tensored by $\left(\tilde{\mathcal{L}}_{r} / \tilde{\mathcal{L}}_{r-1}\right)^{\otimes 1-r}$ we get

$$
\mathcal{F}_{s, k, t, l-1}^{r} \otimes \operatorname{det} \mathcal{L}_{r}^{*}[2-r] \cong \psi_{*}\left(\phi^{*} \mathcal{F}_{s, k, t, l}^{r-1} \otimes\left(\tilde{\mathcal{L}}_{r} / \tilde{\mathcal{L}}_{r-1}\right)^{\otimes 1-r}\right) .
$$

Now we use the first formula and the induction assumption to compute $\mathcal{F}_{s, k, t, l}^{r}$ for $0 \leq k-l \leq r-1-i$ and the second formula and the induction assumption to compute $\mathcal{F}_{s, k, t, l}^{r}$ for $k-l=r-i$. 
Corollary 4.24. If $\mathbf{Z}=\emptyset$ then $\left(F_{1}(1), F_{2}(1), \ldots, F_{1}(N-i), F_{2}(N-i)\right)$ is an exceptional collection on $Y$ and $\operatorname{RHom}\left(F_{1}, F_{2}\right)=W$.

Proof: If $\mathbf{Z}=\emptyset$ then $\mathbf{P}_{N}=$ Speck, $\mathcal{Y}_{N}=Y, g_{N *} \operatorname{RHom} \mathcal{Y}_{N}\left(F_{s}(k), F_{t}(l)\right)=\operatorname{RHom}_{Y}\left(F_{s}(k), F_{t}(l)\right)$.

4.6. Semiorthogonal decompositions for linear section of $Y$. In this subsection we establish semiorthogonal decompositions for $\mathcal{Y}_{r}$ when $r \geq i$.

First of all we have to show that $\Phi_{r}^{!}\left(\mathcal{D}^{b}\left(\mathcal{X}_{r}\right)\right)$ is orthogonal to $F_{s}^{*}(k)$ for $i-r \leq k \leq-1$. This follows from the

Proposition 4.25. If $r \geq i$ and $\mathbf{P}_{r} \neq \emptyset$ then

$$
\Phi_{r}\left(F_{s}^{*}(k)\right) \cong \begin{cases}0, & \text { for } i-r \leq k \leq-1 \\ E_{2-s}(1) \otimes \operatorname{det} \mathcal{L}_{r}[N-r-\operatorname{dim} Y], & \text { for } k=i-r-1\end{cases}
$$

Proof: We use the same arguments as in the proof of proposition 4.23. The base of the induction, $r=i$, follows from proposition 4.21 , since $\Phi_{i}^{!}$is an equivalence by proposition 4.16 . Now assume that the claim is true for $r-1$ and consider the diagram (21). Let us denote

$$
\mathcal{G}_{s, k}^{r}=\Phi_{r}\left(F_{s}^{*}(k)\right), \quad \tilde{\mathcal{G}}_{s, k}^{r}=\tilde{\Phi}_{r}\left(F_{s}^{*}(k)\right) .
$$

First of all we note that

$$
\tilde{\mathcal{G}}_{s, k}^{r-1}=\tilde{\Phi}_{r-1}\left(F_{s}^{*}(k)\right) \cong \tilde{\Phi}_{r-1}\left(\phi^{*} F_{s}^{*}(k)\right) \cong \phi^{*} \Phi_{r-1}\left(F_{s}^{*}(k)\right)=\phi^{*} \mathcal{G}_{s, k}^{r-1},
$$

and similarly

$$
\tilde{\mathcal{G}}_{s, k}^{r} \cong \psi^{*} \mathcal{G}_{s, k}^{r} .
$$

On the other hand, by $4.3(i v)$ we have a resolution

$$
0 \rightarrow F_{s}^{*}(k-1) \otimes\left(\tilde{\mathcal{L}}_{r} / \tilde{\mathcal{L}}_{r-1}\right)^{*} \rightarrow F_{s}^{*}(k) \rightarrow \eta_{*} F_{s}^{*}(k) \rightarrow 0 .
$$

Applying to it the functor $\tilde{\Phi}_{r}$ and taking into account lemma 4.10 we deduce the following exact triangle on $\mathbf{S}_{r}$

$$
\tilde{\mathcal{G}}_{s, k-1}^{r} \otimes\left(\tilde{\mathcal{L}}_{r} / \tilde{\mathcal{L}}_{r-1}\right)^{*} \rightarrow \tilde{\mathcal{G}}_{s, k}^{r} \rightarrow \xi^{*} \tilde{\mathcal{G}}_{s, k}^{r-1} .
$$

Since $\tilde{\mathcal{G}}_{s, k}^{r-1} \cong \phi^{*} \mathcal{G}_{s, k}^{r-1}, \tilde{\mathcal{G}}_{s, k}^{r} \cong \psi^{*} \mathcal{G}_{s, k}^{r}$ we can rewrite this as

$$
\psi^{*}\left(\mathcal{G}_{s, k-1}^{r}\right) \otimes\left(\tilde{\mathcal{L}}_{r} / \tilde{\mathcal{L}}_{r-1}\right)^{*} \rightarrow \psi^{*}\left(\mathcal{G}_{s, k}^{r}\right) \rightarrow \phi^{*} \mathcal{G}_{s, k}^{r-1} .
$$

Finally, recall (40). Applying the functor $\psi_{*}$ to the triangle (39) we get

$$
\mathcal{G}_{s, k}^{r} \cong \psi_{*} \phi^{*} \mathcal{G}_{s, k}^{r-1},
$$

by corollary 4.5 , and applying the functor $\psi_{*}$ to the triangle $(41)$ tensored by $\left(\tilde{\mathcal{L}}_{r} / \tilde{\mathcal{L}}_{r-1}\right)^{\otimes 1-r}$ we get

$$
\mathcal{G}_{s, k-1}^{r} \otimes \operatorname{det} \mathcal{L}_{r}^{*}[2-r] \cong \psi_{*}\left(\phi^{*} \mathcal{G}_{s, k}^{r-1} \otimes\left(\tilde{\mathcal{L}}_{r} / \tilde{\mathcal{L}}_{r-1}\right)^{\otimes 1-r}\right) .
$$

Now we use the first formula and the induction assumption to compute $\mathcal{G}_{s, k}^{r}$ for $i-r \leq k \leq-1$ and the second formula and the induction assumption to compute $\mathcal{G}_{s, k}^{r}$ for $k=i-r-1$.

Now we can check semiorthogonality.

Proposition 4.26. For all $r \geq i$ such that $\mathbf{P}_{r} \neq \emptyset$ the following collection

$$
\left\langle\Phi_{r}^{!}\left(\mathcal{D}^{b}\left(\mathcal{X}_{r}\right)\right), F_{2}^{*}(i-r) \otimes \mathcal{D}^{b}\left(\mathbf{P}_{r}\right), \ldots, F_{1}^{*}(-1) \otimes \mathcal{D}^{b}\left(\mathbf{P}_{r}\right)\right\rangle
$$

is semiorthogonal in $\mathcal{D}^{b}\left(\mathcal{Y}_{r}\right)$. 
Proof: First of all, for all $G, G^{\prime} \in \mathcal{D}^{b}\left(\mathbf{P}_{r}\right)$ we have

$$
\begin{aligned}
\operatorname{Hom}_{\mathcal{Y}_{r}}\left(g_{r}{ }^{*} G\right. & \left.\otimes F_{t}^{*}(l), g_{r}{ }^{*} G^{\prime} \otimes F_{s}^{*}(k)\right) \cong \operatorname{Hom}_{\mathcal{Y}_{r}}\left(g_{r}{ }^{*} G, g_{r}{ }^{*} G^{\prime} \otimes F_{s}^{*}(k) \otimes F_{t}(l)\right) \cong \\
& \left.\cong \operatorname{Hom}_{\mathbf{P}_{r}}\left(G, g_{r *}\left(g_{r}{ }^{*} G^{\prime} \otimes F_{s}^{*}(k) \otimes F_{t}(l)\right)\right) \cong \operatorname{Hom}_{\mathbf{P}_{r}}\left(G, G^{\prime} \otimes g_{r *} \operatorname{RHom}_{\mathcal{Y}_{r}}\left(F_{s}(k), F_{t}(l)\right)\right)\right)
\end{aligned}
$$

and it follows immediately from proposition 4.23 that the functors $G \mapsto g_{r}{ }^{*} G \otimes F_{t}^{*}(l)$ are fully faithful and that the collection $\left\langle F_{2}^{*}(i-r) \otimes \mathcal{D}^{b}\left(\mathbf{P}_{r}\right), \ldots, F_{1}^{*}(-1) \otimes \mathcal{D}^{b}\left(\mathbf{P}_{r}\right)\right\rangle$ in $\mathcal{D}^{b}\left(\mathcal{Y}_{r}\right)$ is semiorthogonal. Further, for any $G \in \mathcal{D}^{b}\left(\mathbf{P}_{r}\right), H \in \mathcal{D}^{b}\left(\mathcal{X}_{r}\right)$, since $\Phi_{r}^{!}$is right adjoint to $\Phi_{r}$ and $\Phi_{r}$ is $\mathbf{S}$-linear we have

$$
\operatorname{Hom}_{\mathcal{Y}_{r}}\left(g_{r}^{*} G \otimes F_{s}^{*}(k), \Phi_{r}^{!}(H)\right) \cong \operatorname{Hom}_{\mathcal{X}_{r}}\left(\Phi_{r}\left(g_{r}^{*} G \otimes F_{s}^{*}(k)\right), H\right) \cong \operatorname{Hom}_{\mathcal{X}_{r}}\left(f_{r}^{*} G \otimes \Phi_{r}\left(F_{s}^{*}(k)\right), H\right)
$$

which is zero for $i-r \leq k \leq-1$ by proposition 4.25. Hence the whole collection is semiorthogonal.

Now we establish semiorthogonal decompositions for the derived categories $\mathcal{D}^{b}\left(\mathcal{Y}_{r}\right)$ with $r \geq i$.

Theorem 4.27. If $r \geq i$ and $\mathbf{P}_{r} \neq \emptyset$ then we have a semiorthogonal decomposition

$$
\mathcal{D}^{b}\left(\mathcal{Y}_{r}\right)=\left\langle\Phi_{r}^{!}\left(\mathcal{D}^{b}\left(\mathcal{X}_{r}\right)\right), F_{2}^{*}(i-r) \otimes \mathcal{D}^{b}\left(\mathbf{P}_{r}\right), \ldots, F_{1}^{*}(-1) \otimes \mathcal{D}^{b}\left(\mathbf{P}_{r}\right)\right\rangle
$$

Proof: By proposition 4.26 it suffices to show that $\Phi_{r}^{!}$is fully faithful and that the RHS in (42) generates $\mathcal{D}^{b}\left(\mathcal{Y}_{r}\right)$. For this we use induction in $r$. The base of induction, $r=i$, is given by proposition 4.16. Assume that the induction hypothesis is true for $r-1$ and let us check that it is also true for $r$. Consider the diagram (21). Then by the faithful base change theorem 2.45 we have a semiorthogonal decomposition

$$
\mathcal{D}^{b}\left(\tilde{\mathcal{Y}}_{r-1}\right)=\left\langle\tilde{\Phi}_{r-1}^{!}\left(\mathcal{D}^{b}\left(\tilde{\mathcal{X}}_{r-1}\right)\right), F_{2}^{*}(i-r+1) \otimes \mathcal{D}^{b}\left(\mathbf{S}_{r}\right), \ldots, F_{1}^{*}(-1) \otimes \mathcal{D}^{b}\left(\mathbf{S}_{r}\right)\right\rangle
$$

By proposition 4.26 we have $\tilde{\Phi}_{r}^{!}\left(\mathcal{D}^{b}\left(\tilde{\mathcal{X}}_{r}\right)\right) \subset\left\langle F_{2}^{*}(-1) \otimes \mathcal{D}^{b}\left(\mathbf{S}_{r}\right), F_{1}^{*}(-1) \otimes \mathcal{D}^{b}\left(\mathbf{S}_{r}\right)\right\rangle^{\perp}$ Using lemma $4.11(i)$ we deduce that $\Phi_{i_{*} \widehat{\mathcal{E}}(0,1) \otimes\left(\tilde{\mathcal{L}}_{r} / \tilde{\mathcal{L}}_{r-1)}\right.} \circ \tilde{\Phi}_{r}^{!}=0$. Therefore, composing exact triangle (28) with $\tilde{\Phi}_{r}^{!}$we obtain an isomorphism $\tilde{\Phi}_{r-1} \eta^{!} \tilde{\Phi}_{r}^{!} \cong \xi_{*} \tilde{\Phi}_{r} \tilde{\Phi}_{r}^{!}$. Applying also lemma 4.10 and the induction hypothesis we deduce

$$
\xi_{*} \tilde{\Phi}_{r} \tilde{\Phi}_{r}^{!} \cong \tilde{\Phi}_{r-1} \eta^{!} \tilde{\Phi}_{r}^{!} \cong \tilde{\Phi}_{r-1} \tilde{\Phi}_{r-1}^{!} \xi_{*} \cong \xi_{*} .
$$

Then by lemma $2.8 b$ ) we have $\tilde{\Phi}_{r} \tilde{\Phi}_{r}^{!} \cong \mathrm{id}_{\tilde{\mathcal{X}}_{r}}$, and by lemma 2.41 and corollary 4.5 we have

$$
\Phi_{r} \Phi_{r}^{!} \cong \psi_{*} \psi^{*} \Phi_{r} \Phi_{r}^{!} \cong \psi_{*} \tilde{\Phi}_{r} \psi^{*} \Phi_{r}^{!} \cong \psi_{*} \tilde{\Phi}_{r} \tilde{\Phi}_{r}^{!} \psi^{*} \cong \psi_{*} \psi^{*} \cong \mathrm{id} \mathcal{X}_{r},
$$

hence $\Phi_{r}^{!}$is fully faithful.

Finally, assume that $G \in \mathcal{D}^{b}\left(\mathcal{Y}_{r}\right)$ is in the left orthogonal to the RHS of (42), so that

$$
\operatorname{Hom}_{\mathcal{Y}_{r}}\left(G, F_{s}^{*}(k)\right)=0 \quad \text { for } i-r \leq k \leq-1 \quad \text { and } \quad \Phi_{r}(G)=0 .
$$

Since $\hat{\psi}^{*}$ is fully faithful by corollary 4.5 we have for all $i-r+1 \leq k \leq-1$

$$
\operatorname{Hom}_{\tilde{\mathcal{Y}}_{r-1}}\left(\hat{\psi}^{*} G(1), F_{s}^{*}(k)\right) \cong \operatorname{Hom}_{\tilde{\mathcal{Y}}_{r-1}}\left(\hat{\psi}^{*} G, \hat{\psi}^{*} F_{s}^{*}(k-1)\right) \cong \operatorname{Hom}_{\mathcal{Y}_{r}}\left(G, F_{s}^{*}(k-1)\right)=0 .
$$

On the other hand, since

$$
\hat{\psi}^{*} G(1) \cong \eta^{*} \psi^{*} G(1) \cong \eta^{!} \psi^{*} G \otimes\left(\tilde{\mathcal{L}}_{r} / \tilde{\mathcal{L}}_{r-1}\right)^{*}[1]
$$

by lemma 4.3 (iv) and $\psi^{*} G \in\left\langle F_{2}^{*}(-1) \otimes \mathcal{D}^{b}\left(\mathbf{S}_{r}\right), F_{1}^{*}(-1) \otimes \mathcal{D}^{b}\left(\mathbf{S}_{r}\right)\right\rangle^{\perp}$ by fullness and faithfulness of $\psi^{*}$ (corollary 4.5), we have $\Phi_{i_{*} \widehat{\mathcal{E}}(0,1) \otimes\left(\tilde{\mathcal{L}}_{r} / \tilde{\mathcal{L}}_{r-1}\right)}\left(\psi^{*} G\right)=0$ by lemma 4.11. Taking into account (28) we deduce

$$
\begin{aligned}
\tilde{\Phi}_{r-1}\left(\hat{\psi}^{*} G(1)\right) \cong \tilde{\Phi}_{r-1}\left(\eta^{!} \psi^{*} G \otimes\left(\tilde{\mathcal{L}}_{r} / \tilde{\mathcal{L}}_{r-1}\right)^{*}[1]\right) \cong\left(\tilde{\Phi}_{r-1} \eta^{!} \psi^{*} G\right) \otimes\left(\tilde{\mathcal{L}}_{r} / \tilde{\mathcal{L}}_{r-1}\right)^{*}[1] \cong \\
\\
\cong\left(\xi_{*} \tilde{\Phi}_{r} \psi^{*} G\right) \otimes\left(\tilde{\mathcal{L}}_{r} / \tilde{\mathcal{L}}_{r-1}\right)^{*}[1] \cong\left(\xi_{*} \psi^{*} \Phi_{r}(G) \otimes\left(\tilde{\mathcal{L}}_{r} / \tilde{\mathcal{L}}_{r-1}\right)^{*}[1]=0\right.
\end{aligned}
$$

by (44). Thus $\hat{\psi}^{*} G(1)$ is in the left orthogonal to the RHS of $(43)$, hence $\hat{\psi}^{*} G(1)=0$. But then $G(1)=\hat{\psi}_{*} \hat{\psi}^{*} G(1)=0$ and $G=0$. Thus the induction claim is proved. 
4.7. Proof of the main theorem. Let $L \subset V^{*}$ be an admissible subspace, $\operatorname{dim} L=r$.

Lemma 4.28. The map $\varphi:$ Speck $\rightarrow \mathbf{P}_{r}$ induced by $L$ is a faithful base change for the pair $\left(\mathcal{X}_{r}, \mathcal{Y}_{r}\right)$.

Proof: Use lemma 2.27, 4.3, 4.1 and the definition of admissible subspace.

Now we apply the faithful base change theorem 2.45. Then theorem 1.2 follows from theorem 4.19 and theorem 4.27 .

Corollary 4.29. Assume that $L \subset V^{*}$ is an admissible subspace. Then $Y_{L}$ is smooth iff $X_{L}$ is smooth.

Proof: Note that lemma D.22 implies that $X_{L}$ is smooth iff the category $\mathcal{D}^{b}\left(X_{L}\right)$ is Ext-bounded, and $Y_{L}$ is smooth iff the category $\mathcal{D}^{b}\left(Y_{L}, \mathcal{A}_{Y}\right)$ is Ext-bounded. On the other hand, one of these categories is embedded fully and faithfully into another, and the orthogonal is generated by an exceptional collection. Hence $\mathcal{D}^{b}\left(X_{L}\right)$ is Ext-bounded iff $\mathcal{D}^{b}\left(Y_{L}, \mathcal{A}_{Y}\right)$ is Ext-bounded.

Let $\operatorname{sing}(g) \subset \mathbb{P}\left(V^{*}\right) \backslash \mathbf{Z}$ denote the set of critical values of $g: Y \rightarrow \mathbb{P}\left(V^{*}\right) \backslash \mathbf{Z}$. Let $X^{\vee} \subset \mathbb{P}\left(V^{*}\right)$ be the projectively dual variety of $X$.

Corollary 4.30. If $g$ is flat then $\operatorname{sing}(g)=X^{\vee} \backslash \mathbf{Z}$.

Proof: If $g$ is flat then every 1-dimensional subspace $H \subset V^{*}$ is admissible hence

$$
\operatorname{sing}(g)=\left\{H \in \mathbb{P}\left(V^{*}\right) \backslash \mathbf{Z} \mid Y_{H} \text { is singular }\right\}=\left\{H \in \mathbb{P}\left(V^{*}\right) \backslash \mathbf{Z} \mid X_{H} \text { is singular }\right\}=X^{\vee} \backslash \mathbf{Z} .
$$

\section{General construction of $Y$}

In this section we will give a general approach to construction of the Azumaya variety $Y$ for a given $X$. The approach uses moduli spaces of quiver representations. Our general reference for this subject is $[\mathrm{Ki}]$. We will freely use conventions and notations thereof.

So, assume that we are given the data (D.1) and (D.2) satisfying the conditions (C.1)-(C.3). Assume additionally that

$$
\operatorname{Ext}^{>0}\left(E_{2}(-1), E_{1}\right)=\operatorname{Ext}^{>0}\left(E_{2}(-1), E_{2}\right)=0 .
$$

Consider a quiver $\mathrm{Q}=\bullet \stackrel{W}{\longrightarrow} \bullet$ with two vertices and $\operatorname{dim} W$ arrows from the first vertex to the second, and a slope function $\theta: K_{0}(\mathrm{Q}) \rightarrow \mathbb{Z}$, given by the formula

$$
\theta\left(d_{1}, d_{2}\right)=d_{2} \operatorname{rank}\left(E_{2}\right)-d_{1} \operatorname{rank}\left(E_{1}\right) .
$$

For every dimension vector $\left(d_{1}, d_{2}\right) \in K_{0}(\mathrm{Q})$, take a pair of vector spaces $R_{1}, R_{2}$ of dimensions $d_{1}$ and $d_{2}$ respectively, and consider an affine space $\mathbf{R}=\mathbf{R}_{\mathbf{Q}}\left(d_{1}, d_{2}\right)=\operatorname{Hom}\left(R_{1} \otimes W, R_{2}\right)$ parameterizing all $\left(d_{1}, d_{2}\right)$-dimensional representations of $\mathbf{Q}$. We denote the trivial vector bundles on $\mathbf{R}$ with fibers $R_{1}$ and $R_{2}$ by $\mathcal{R}_{1}, \mathcal{R}_{2}$. Then on $\mathbf{R}$ we have a universal representation of the quiver $\underline{\rho}: W \otimes \mathcal{R}_{1} \rightarrow \mathcal{R}_{2}$. Consider on $X \times \mathbf{R}$ a map $e: E_{1} \otimes \mathcal{R}_{1} \rightarrow E_{2} \otimes \mathcal{R}_{2}$ defined as the composition

$$
e: E_{1} \otimes \mathcal{R}_{1} \stackrel{\underline{\rho}^{*}}{\longrightarrow} E_{1} \otimes\left(W^{*} \otimes \mathcal{R}_{2}\right) \cong\left(W^{*} \otimes E_{1}\right) \otimes \mathcal{R}_{2} \stackrel{\text { ev }}{\longrightarrow} E_{2} \otimes \mathcal{R}_{2},
$$

where ev : $W^{*} \otimes E_{1}=\operatorname{Hom}\left(E_{1}, E_{2}\right) \otimes E_{1} \rightarrow E_{2}$ is the evaluation homomorphism. For each $\rho \in \mathbf{R}$ we denote by $e_{\rho}$ the restriction of $e$ to $X \cong X \times\{\rho\} \subset X \times \mathbf{R}$. Let

$$
C=\text { Coker } e, \quad C_{\rho}=\text { Coker } e_{\rho} \cong C_{\mid X \times\{\rho\}} .
$$


An embedding $V^{*} \subset H^{0}\left(X, \mathcal{O}_{X}(1)\right) \subset \operatorname{Hom}\left(E_{2}(-1) \otimes \mathcal{R}_{2}, E_{2} \otimes \mathcal{R}_{2}\right)$ combined with the projection $E_{2} \otimes$ $\mathcal{R}_{2} \rightarrow C$ induces a morphism $V^{*} \otimes \mathcal{O}_{X \times \mathbf{R}} \rightarrow\left(E_{2}^{*}(1) \otimes \mathcal{R}_{2}^{*}\right) \otimes C$. Taking a pushforward with respect to the projection $q: X \times \mathbf{R} \rightarrow \mathbf{R}$ we obtain a homomorphism

$$
\nu: V^{*} \otimes \mathcal{O}_{\mathbf{R}} \rightarrow R^{0} q_{*}\left(\left(E_{2}^{*}(1) \otimes \mathcal{R}_{2}^{*}\right) \otimes C\right) .
$$

From now on assume that

$$
\theta\left(d_{1}, d_{2}\right)=0 .
$$

This condition determines the dimension vector $\left(d_{1}, d_{2}\right)$ up to a constant. Let $\operatorname{gcd}\left(d_{1}, d_{2}\right)=m$ and denote the corresponding representation space $\mathbf{R}_{\mathbf{Q}}\left(d_{1}, d_{2}\right)$ by $\mathbf{R}_{m}$. Consider the following subschemes of $\mathbf{R}_{m}$ :

- the $\theta$-stable locus $\mathbf{R}_{m}^{s}$;

- the $\theta$-semistable locus $\mathbf{R}_{m}^{s s}$;

- an open subscheme $\mathbf{R}_{m}^{\circ}=\left\{\rho \in \mathbf{R}_{m} \mid \operatorname{Ker} e_{\rho}=0\right\} \subset \mathbf{R}_{m}$;

- a subset $\mathbf{R}_{m}^{1}=\left\{\rho \in \mathbf{R}_{m} \mid \operatorname{Ker} \nu_{\rho} \neq 0\right\} \subset \mathbf{R}_{m}$;

- a subset $\tilde{\mathbf{R}}_{m}^{1}=\left\{(\rho, H) \in \mathbf{R}_{m} \times \mathbb{P}\left(V^{*}\right) \mid \nu_{\rho}(H)=0\right\} \subset \mathbf{R}_{m} \times \mathbb{P}\left(V^{*}\right)$.

Proposition 5.1. We have $\tilde{\mathbf{R}}_{m}^{1} \subset \mathbf{R}_{m}^{1} \times \mathbb{P}\left(V^{*}\right)$ and $\mathbf{R}_{m}^{1} \subset \mathbf{R}_{m}^{\circ} \subset \mathbf{R}_{m}^{s s}$. Moreover, the sheaf $R^{0} q_{*}\left(\left(E_{2}^{*}(1) \otimes\right.\right.$ $\left.\mathcal{R}_{2}^{*}\right) \otimes C$ ) is locally free on $\mathbf{R}_{m}^{\circ}$.

Proof: The first embedding is evident. Assume that $\rho \in \mathbf{R}_{m}^{1}$ and $0 \neq H \in \operatorname{Ker} \nu_{\rho}$. Note that the composition of $\nu_{\rho}$ with the canonical map

$$
\left(R^{0} q_{*}\left(\left(E_{2}^{*}(1) \otimes \mathcal{R}_{2}^{*}\right) \otimes C\right)\right)_{\rho} \rightarrow H^{0}\left(X,\left(E_{2}^{*}(1) \otimes \mathcal{R}_{2}^{*}\right) \otimes C_{\rho}\right) \cong \operatorname{Hom}\left(E_{2}(-1) \otimes R_{2}, C_{\rho}\right)
$$

coincides with the composition $V^{*} \rightarrow \operatorname{Hom}\left(E_{2}(-1) \otimes R_{2}, E_{2} \otimes R_{2}\right) \rightarrow \operatorname{Hom}\left(E_{2}(-1) \otimes R_{2}, C_{\rho}\right)$. Therefore, the assumption implies that the dashed arrow in the diagram

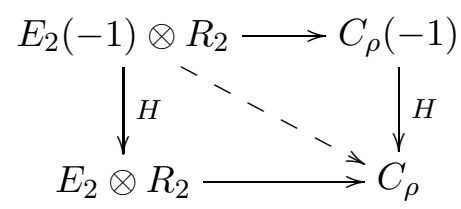

vanishes. But since the upper arrow is surjective it follows that the map $C_{\rho}(-1) \stackrel{H}{\longrightarrow} C_{\rho}$ is zero, hence $C$ is supported scheme-theoretically on the hyperplane section $X_{H}$ of $X$.

$$
\rho \in \mathbf{R}_{m}^{1} \quad \text { if and only if } \quad \operatorname{supp}\left(C_{\rho}\right) \subset H \in|\mathcal{H}|,
$$

where $|\mathcal{H}|$ is the linear system of hyperplane sections of $X \subset \mathbb{P}(V)$. In particular, we have $\operatorname{rank}\left(C_{\rho}\right)=0$, hence $\operatorname{rank} \operatorname{Ker} e_{\rho}=\operatorname{rank}\left(E_{1} \otimes R_{1}\right)-\operatorname{rank}\left(E_{2} \otimes R_{2}\right)=-\theta\left(d_{1}, d_{2}\right)=0$, hence $e_{\rho}$ is a monomorphism, so $\mathbf{R}_{m}^{1} \subset \mathbf{R}_{m}^{\circ}$. Moreover, we have the following exact sequence

$$
0 \rightarrow E_{1} \otimes R_{1} \stackrel{e_{\rho}}{\longrightarrow} E_{2} \otimes R_{2} \rightarrow C_{\rho} \rightarrow 0
$$

for any $\rho \in \mathbf{R}_{m}^{1}$. Note also that $e_{\mid X \times \mathbf{R}_{m}^{\circ}}$ is also a monomorphism and we have on $X \times \mathbf{R}_{m}^{\circ}$ the following exact sequence

$$
0 \rightarrow E_{1} \otimes \mathcal{R}_{1} \stackrel{e}{\longrightarrow} E_{2} \otimes \mathcal{R}_{2} \rightarrow C \rightarrow 0
$$

Now let us check that $\mathbf{R}_{m}^{\circ} \subset \mathbf{R}_{m}^{s s}$. Assume that $\rho \in \mathbf{R}_{m}^{\circ}$ is unstable. Then there exist subspaces $R_{1}^{\prime} \subset R_{1}, R_{2}^{\prime} \subset R_{2}$, such that $\theta\left(\operatorname{dim} R_{1}^{\prime}, \operatorname{dim} R_{2}^{\prime}\right)<0$. Then

$$
\operatorname{rank} \operatorname{Ker} e_{\rho^{\prime}} \geq \operatorname{rank}\left(E_{1} \otimes R_{1}^{\prime}\right)-\operatorname{rank}\left(E_{2} \otimes R_{2}^{\prime}\right)=-\theta\left(\operatorname{dim} R_{1}^{\prime}, \operatorname{dim} R_{2}^{\prime}\right)>0,
$$

hence $\operatorname{Ker}\left(e_{\rho^{\prime}}: E_{1} \otimes R_{1}^{\prime} \rightarrow E_{2} \otimes R_{2}^{\prime}\right) \neq 0$. On the other hand, it is clear that $\operatorname{Ker} e_{\rho^{\prime}} \subset \operatorname{Ker} e_{\rho}=0$, which gives a contradiction. 
Comparing (49) and (50) we see that $C \otimes \mathcal{O}_{X \times \rho} \cong C_{\rho}$. It follows that the sheaf $C$ (and therefore $\left(E_{2}^{*}(1) \otimes \mathcal{R}_{2}^{*}\right) \otimes C$ as well) is flat over $\mathbf{R}_{m}^{\circ}$. Furthermore, using (49) and taking into account (45) we deduce that $H^{p}\left(X,\left(E_{2}^{*}(1) \otimes R_{2}^{*}\right) \otimes C_{\rho}\right)=0$ for $p>0$ and the dimension of the zero cohomology doesn't depend on $\rho$. Finally, for any closed point $\rho \in \mathbf{R}_{m}^{\circ}$ we have

$$
q_{*}\left(\left(E_{2}^{*}(1) \otimes \mathcal{R}_{2}^{*}\right) \otimes C\right) \otimes \mathcal{O}_{\rho} \cong H^{\bullet}\left(X,\left(E_{2}^{*}(1) \otimes R_{2}^{*}\right) \otimes C \otimes \mathcal{O}_{X \times \rho}\right) .
$$

Therefore, $q_{*}\left(\left(E_{2}^{*}(1) \otimes \mathcal{R}_{2}^{*}\right) \otimes C\right)=R^{0} q_{*}\left(\left(E_{2}^{*}(1) \otimes \mathcal{R}_{2}^{*}\right) \otimes C\right)$ is a vector bundle.

Lemma 5.2. If

$$
\operatorname{codim}_{\mathbf{R}_{m}}\left(\mathbf{R}_{m} \backslash \mathbf{R}_{m}^{\circ}\right) \geq 2
$$

then $\mathbf{R}_{m}^{1}$ is closed in $\mathbf{R}_{m}$ and $\tilde{\mathbf{R}}_{m}^{1}$ is closed in $\mathbf{R}_{m} \times \mathbb{P}\left(V^{*}\right)$.

Proof: It is clear that $\tilde{\mathbf{R}}_{m}^{1}$ is closed in $\mathbf{R}_{m}^{1} \times \mathbb{P}\left(V^{*}\right)$ and $\mathbf{R}_{m}^{1}$ is the degeneration locus of a the morphism $\nu: V^{*} \otimes \mathcal{O}_{\mathbf{R}_{m}} \rightarrow R^{0} q_{*}\left(\left(E_{2}^{*}(1) \otimes \mathcal{R}_{2}^{*}\right) \otimes C\right)$, so it suffices to check that the sheaf $R^{0} q_{*}\left(\left(E_{2}^{*}(1) \otimes \mathcal{R}_{2}^{*}\right) \otimes C\right)$ is torsion free. It follows from (45) and (50) that we have the following exact sequence on $\mathbf{R}_{m}$ :

$$
0 \rightarrow \operatorname{Hom}\left(E_{2}(-1), E_{1}\right) \otimes \mathcal{R}_{2}^{*} \otimes \mathcal{R}_{1} \rightarrow \operatorname{Hom}\left(E_{2}(-1), E_{2}\right) \otimes \mathcal{R}_{2}^{*} \otimes \mathcal{R}_{2} \rightarrow R^{0} q_{*}\left(\left(E_{2}^{*}(1) \otimes \mathcal{R}_{2}^{*}\right) \otimes C\right) \rightarrow 0,
$$

so the sheaf admits a length 2 locally free resolution. Therefore, it is torsion free if and only if it is locally free in codimension 2 (see [OSS]). But we have proved in proposition 5.1 that it is locally free on $\mathbf{R}_{m}^{\circ}$.

The following lemma is useful for verification of (51).

Lemma 5.3. If $\operatorname{codim}_{\mathbf{R}_{1}}\left(\mathbf{R}_{1} \backslash \mathbf{R}_{1}^{\circ}\right) \geq 2$ then $\operatorname{codim}_{\mathbf{R}_{m}}\left(\mathbf{R}_{m} \backslash \mathbf{R}_{m}^{\circ}\right) \geq 2$.

Proof: Consider the embedding $\left(\mathbf{R}_{1}\right)^{m} \rightarrow \mathbf{R}_{m},\left(\rho_{1}, \ldots, \rho_{m}\right) \mapsto \rho_{1} \oplus \cdots \oplus \rho_{m}$. Since $\mathbf{R}_{m}$ is a vector space and $\mathbf{R}_{m}^{\circ}$ is dilations-invariant, it suffices to check that $\operatorname{codim}_{\left(\mathbf{R}_{1}\right)^{m}}\left(\left(\mathbf{R}_{1}\right)^{m} \backslash\left(\mathbf{R}_{m}^{\circ} \cap\left(\mathbf{R}_{1}\right)^{m}\right)\right) \geq 2$. But it is clear that $\left(\mathbf{R}_{m}^{\circ} \cap\left(\mathbf{R}_{1}\right)^{m}\right)=\left(\mathbf{R}_{1}^{\circ}\right)^{m}$ and the claim follows.

For any divisor class $D$ such that both linear systems $|D|$ and $|\mathcal{H}-D|$ are nonempty let $Z^{|D|} \subset \mathbb{P}\left(V^{*}\right)$ denote the image of $|D| \times|\mathcal{H}-D|$ in $|\mathcal{H}|=\mathbb{P}\left(V^{*}\right)$. Let $\tilde{g}_{m}$ denote the projection $\tilde{\mathbf{R}}_{m}^{1} \rightarrow \mathbb{P}\left(V^{*}\right)$ and let $p$ denote the projection $\tilde{\mathbf{R}}_{m}^{1} \rightarrow \mathbf{R}_{m}^{1}$. Consider the open subset

$$
U:=\tilde{\mathbf{R}}_{m}^{1} \backslash \underset{0<D<\mathcal{H}}{\cup} \tilde{g}_{m}^{-1}\left(Z^{|D|}\right) \subset \tilde{\mathbf{R}}_{m}^{1} .
$$

Proposition 5.4. The projection $p: U \rightarrow p(U) \subset \mathbf{R}_{m}^{1}$ is an isomorphism.

Proof: Let us check that $\operatorname{Ker} \nu_{\mid p(U)}$ is a line subbundle in $V^{*} \otimes \mathcal{O}_{p(U)}$. Since $\mathbf{R}_{m}^{1}$ is the degeneration locus of a morphism of vector bundles $\nu: V^{*} \otimes \mathcal{O}_{\mathbf{R}_{m}^{\circ}} \rightarrow R^{0} q_{*}\left(\left(E_{2}^{*}(1) \otimes \mathcal{R}_{2}^{*}\right) \otimes C\right)$ on $\mathbf{R}_{m}^{\circ}$ it suffices to check that $\operatorname{dim} \operatorname{Ker} \nu_{\rho}=1$ for all $\rho \in p(U)$. Indeed, assume that $\operatorname{dim} \operatorname{Ker} \nu_{\rho} \geq 2$ and let $\left\langle H, H^{\prime}\right\rangle$ be a 2-dimensional subspace in $\operatorname{Ker} \nu_{\rho}$. Then (48) implies that $\operatorname{supp}\left(C_{\rho}\right) \subset H \cap H^{\prime}$. On the other hand, it follows from (49) that either $C_{\rho}=0$, which would imply that $\operatorname{Hom}\left(E_{2}, E_{1}\right) \neq 0$ thus contradicting (C.3), or that $D=\operatorname{supp}\left(C_{\rho}\right)$ is a divisor in $X$. Since $D \subset H \cap H^{\prime}$, we have $H \in Z^{|D|}$ and $(\rho, H) \in \tilde{g}_{m}^{-1}\left(Z^{|D|}\right)$.

Consider a map $p(U) \rightarrow \mathbb{P}\left(V^{*}\right)$ given by the line subbundle $\operatorname{Ker} \nu_{\mid p(U)} \subset V^{*} \otimes \mathcal{O}_{\mid p(U)}$. Combining it with the embedding $p(U) \subset \mathbf{R}_{m}^{1}$ we obtain a map to $p(U) \rightarrow U \subset \mathbf{R}_{m}^{1} \times \mathbb{P}\left(V^{*}\right)$, which is clearly the inverse to the projection $p$.

Consider the group $G=\left(\mathrm{GL}\left(R_{1}\right) \times \mathrm{GL}\left(R_{2}\right)\right) / \mathrm{k}^{*}$. Let $\chi: G \rightarrow \mathrm{k}^{*}$ denote the character

$$
\chi\left(g_{1}, g_{2}\right)=\operatorname{det}\left(g_{1}\right)^{-\operatorname{rank}\left(E_{1}\right)} \operatorname{det}\left(g_{2}\right)^{\operatorname{rank}\left(E_{2}\right)},
$$

corresponding to the slope function (46). Consider the action of $G$ on $\mathbf{R}_{m}$ by isomorphisms of representations linearized by the character $\chi$, and the trivial action of $G$ on $\mathbb{P}\left(V^{*}\right)$. Note that $\mathbf{R}_{m}^{1} \subset \mathbf{R}_{m}^{s s}$ and $\tilde{\mathbf{R}}_{m}^{1} \subset \mathbf{R}_{m}^{s s} \times \mathbb{P}\left(V^{*}\right)$ are $G$-invariant closed subschemes (if (51) is satisfied). Consider the following GIT quotients: 
- $\mathcal{M}_{m}^{s s}=\mathbf{R}_{m}^{s s} / / \chi G$, the moduli space of semistable representations;

- $\mathcal{M}_{m}^{s}=\mathbf{R}_{m}^{s} / / \chi G \subset \mathcal{M}_{m}^{s s}$, the moduli space of stable representations;

- $\bar{Y}_{m}=\tilde{\mathbf{R}}_{m}^{1} / / \chi G \subset \mathcal{M}_{m}^{s s} \times \mathbb{P}\left(V^{*}\right)$;

Let $\bar{g}_{m}: \bar{Y}_{m} \rightarrow \mathbb{P}\left(V^{*}\right)$ denote the projection to $\mathbb{P}\left(V^{*}\right)$. Let

$$
Z_{m}=\bar{g}_{m}\left(\bar{Y}_{m}\right) \subset \mathbb{P}\left(V^{*}\right), \quad \bar{Z}_{m}=\left(\bigcup_{k=1}^{[m / 2]} Z_{k}\right) \cup\left(\bigcup_{0<D<\mathcal{H}} Z^{|D|}\right) .
$$

Then $\bar{Z}_{m}$ is closed in $\mathbb{P}\left(V^{*}\right)$. Consider

$$
Y_{m}=\bar{Y}_{m} \backslash \bar{g}_{m}^{-1}\left(\bar{Z}_{m}\right)=\bar{g}_{m}^{-1}\left(\mathbb{P}\left(V^{*}\right) \backslash \bar{Z}_{m}\right)
$$

and let $g_{m}: Y_{m} \rightarrow \mathbb{P}\left(V^{*}\right)$ denote the restriction of $\bar{g}_{m}$ to $Y_{m}$.

Lemma 5.5. The map $g_{m}: Y_{m} \rightarrow \mathbb{P}\left(V^{*}\right) \backslash \bar{Z}_{m}$ is projective and the projection $Y_{m} \rightarrow \mathcal{M}_{m}^{s}$ is an embedding.

Proof: Since $\tilde{\mathbf{R}}_{m}^{1}$ is closed in $\mathbf{R}_{m}^{s s} \times \mathbb{P}\left(V^{*}\right)$ it follows that $\bar{Y}_{m}$ is closed in $\mathcal{M}_{m}^{s s} \times \mathbb{P}\left(V^{*}\right)$, which is a projective variety by results of $[\mathrm{Ki}]$. Therefore, $\bar{Y}_{m}$ is projective and the map $\bar{g}_{m}$ is projective as well. On the other hand, the map $g_{m}$ is obtained from $\bar{g}_{m}$ by a change of base $\mathbb{P}\left(V^{*}\right) \backslash \bar{Z}_{m} \rightarrow \mathbb{P}\left(V^{*}\right)$, hence it is also projective. Further note that any $\left(d_{1}, d_{2}\right)$-dimensional strictly semistable representation $\rho$ of $\mathrm{Q}$ with $\operatorname{gcd}\left(d_{1}, d_{2}\right)=m$ contains a $\left(d_{1}^{\prime}, d_{2}^{\prime}\right)$-dimensional grading factors with $\operatorname{gcd}\left(d_{1}^{\prime}, d_{2}^{\prime}\right) \leq[m / 2]$ in its Jordan-Gölder filtration. Hence $\bar{g}_{m}(\rho) \in \bar{Z}_{m}$ and $\rho \notin Y_{m}$. So, the projection of $Y_{m}$ in $\mathcal{M}_{m}^{s s}$ is contained in $\mathcal{M}_{m}^{s}$. Moreover, by proposition 5.4 this projection is an embedding (compare the definition of $Y_{m} \subset \bar{Y}_{m}$ and $U \subset \tilde{\mathbf{R}}_{m}^{1}$ ).

Lemma 5.6. There exists a sheaf of Azumaya algebras $\mathcal{A}_{m}$ on $\mathcal{M}_{m}^{s}$, such that the universal family $\left(\mathcal{R}_{1}, \mathcal{R}_{2}\right)$ of representations of the quiver $\mathrm{Q}$ on $\mathbf{R}_{m}^{s}$ descends to a universal family $\left(F_{1}, F_{2}\right)$ in the category of coherent $\mathcal{A}_{m}$-modules on $\mathcal{M}_{m}^{s}$. Moreover, we have a right exact sequence

$$
W \otimes F_{2}^{*} \otimes F_{1} \rightarrow F_{2}^{*} \otimes F_{2} \oplus F_{1}^{*} \otimes F_{1} \rightarrow \Delta_{*} \mathcal{A}_{\mathcal{M}} \rightarrow 0
$$

on $\mathcal{M}_{m}^{s} \times \mathcal{M}_{m}^{s}$

Proof: Since the action of $G$ on $\mathbf{R}_{m}^{s}$ is free every $\mathrm{GL}\left(R_{1}\right) \times \mathrm{GL}\left(R_{2}\right)$-equivariant vector bundle on $\mathbf{R}_{m}^{s}$ descends to $\mathcal{M}_{m}^{s}$ iff the diagonal subgroup $\mathrm{k}^{*} \subset \mathrm{GL}\left(R_{1}\right) \times \mathrm{GL}\left(R_{2}\right)$ acts on it trivially. However, the diagonal subgroup acts on both $\mathcal{R}_{1}$ and $\mathcal{R}_{2}$ with weight 1 . To get rid of this action we choose one more representation $R_{0}$ of the group $\mathrm{GL}\left(R_{1}\right) \times \mathrm{GL}\left(R_{2}\right)$, on which the diagonal subgroup acts with weight 1 and consider the trivial vector bundle $\mathcal{R}_{0}$ with fiber $R_{0}$ on $\mathbf{R}$. Then the diagonal subgroup acts trivially on $\left(\mathcal{R}_{0}^{*} \otimes \mathcal{R}_{1}, \mathcal{R}_{0}^{*} \otimes \mathcal{R}_{2}\right)$, hence this family descends to $\mathcal{M}_{m}^{s}=\mathbf{R}_{m}^{s} / / \chi G$ and gives a family of $\left(F_{1}, F_{2}\right)$ of representations of the quiver $\mathrm{Q}$ on $\mathcal{M}_{m}^{s}$, which is quasiuniversal. Moreover, $\mathcal{E} n d\left(\mathcal{R}_{0}\right)$ is a sheaf of $G$-equivariant Azumaya algebras, which descends to a sheaf of Azumaya algebras $\mathcal{A}_{m}$ over $\mathcal{M}_{m}^{s}$. It is clear that $\left(F_{1}, F_{2}\right)$ is a universal family of representations of the quiver $\mathrm{Q}$ in the category of coherent $\mathcal{A}_{m}$-modules.

It remains to check that the above sequence is right exact. The action of the group $G$ on $\mathbf{R}_{m}^{s}$ gives a map $\pi: \mathbf{R}_{m}^{s} \times G \rightarrow \mathbf{R}_{m}^{s} \times \mathbf{R}_{m}^{s},(\rho, g) \mapsto(\rho, g \rho)$. Let us check that the complex

$$
\mathcal{C}=\left\{W \otimes \mathcal{R}_{2}^{*} \otimes \mathcal{R}_{1} \stackrel{\operatorname{ad}(\underline{\rho})}{\longrightarrow} \mathcal{R}_{2}^{*} \otimes \mathcal{R}_{2} \oplus \mathcal{R}_{1}^{*} \otimes \mathcal{R}_{1} \longrightarrow \pi_{*} \mathcal{O}\right\},
$$

on $\mathbf{R}_{m}^{s} \times \mathbf{R}_{m}^{s}$ is acyclic in the middle and right term. For any point $\left(\rho_{1}, \rho_{2}\right) \in \mathbf{R}_{m} \times \mathbf{R}_{m}$ we have an exact sequence

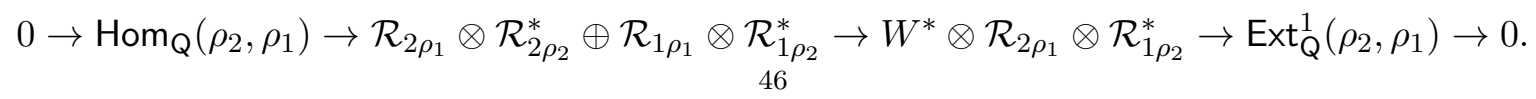


Since both $\rho_{1}$ and $\rho_{2}$ are stable, we have $\operatorname{Hom}_{\mathbf{Q}}\left(\rho_{2}, \rho_{1}\right)=0$ for $\left(\rho_{1}, \rho_{2}\right) \notin \pi\left(\mathbf{R}_{m}^{s} \times G\right)$, and $\operatorname{Hom}_{\mathbf{Q}}\left(\rho_{2}, \rho_{1}\right)=\mathrm{k}$ for $\left(\rho_{1}, \rho_{2}\right) \in \pi\left(\mathbf{R}_{m}^{s} \times G\right)$. Therefore, $\mathcal{H}^{0}(\mathcal{C})$ and $\mathcal{H}^{-1}(\mathcal{C})$ are supported set-theoretically on $\pi\left(\mathbf{R}_{m}^{s} \times G\right)$.

Now compare two spectral sequences computing $\mathcal{H}^{n}\left(\pi^{*} \mathcal{C}\right)$. The first term of the first spectral sequence $E_{1}^{p, q}=\mathcal{H}^{q}\left(\pi^{*}\left(\mathcal{C}^{p}\right)\right) \Longrightarrow \mathcal{H}^{p+q}\left(\pi^{*} \mathcal{C}\right)$ has form

$$
\begin{array}{ccc}
W \otimes \mathcal{R}_{2}^{*} \otimes \mathcal{R}_{1} \stackrel{\operatorname{ad}(\underline{\rho})}{\longrightarrow} \underline{\mathcal{R}}_{2}^{*} \otimes \mathcal{R}_{2} \oplus \mathcal{R}_{1}^{*} \otimes \mathcal{R}_{1} & \multicolumn{1}{c}{\mathcal{O}} \\
0 & 0 & \mathcal{H}^{-1}\left(\pi^{*} \pi_{*} \mathcal{O}\right) \\
0 & 0 & \mathcal{H}^{-2}\left(\pi^{*} \pi_{*} \mathcal{O}\right)
\end{array}
$$

Note that the top line is acyclic in the middle and right term, its cohomology in the left term at a point $(\rho, g) \in \mathbf{R}_{m}^{s} \times G$ is equal to $\operatorname{Ext}_{\mathbf{Q}}^{1}(\rho, \rho)^{*} \cong T_{\rho}^{*} \mathcal{M}_{m}^{s} \cong\left(\mathcal{H}^{-1}\left(\pi^{*} \pi_{*} \mathcal{O}\right)\right)_{(\rho, g)}$, and it is clear that the dashed arrow is an isomorphism. It follows that $\pi^{*} \mathcal{C} \in \mathcal{D}^{\leq-2}\left(\mathbf{R}_{m}^{s} \times G\right)$. Then the second spectral sequence $E_{2}^{p, q}=\mathcal{H}^{q}\left(\pi^{*}\left(\mathcal{H}^{p}(\mathcal{C})\right)\right) \Longrightarrow \mathcal{H}^{p+q}\left(\pi^{*} \mathcal{C}\right)$ implies that $\mathcal{H}^{0}\left(\pi^{*} \mathcal{H}^{0}(\mathcal{C})\right)=\mathcal{H}^{0}\left(\pi^{*} \mathcal{H}^{-1}(\mathcal{C})\right)=0$, hence $\mathcal{H}^{0}(\mathcal{C})=\mathcal{H}^{-1}(\mathcal{C})=0$ since both are supported set-theoretically on $\pi\left(\mathbf{R}_{m}^{s} \times G\right)$. Tensoring $\mathcal{C}$ with $\mathcal{R}_{0} \otimes \mathcal{R}_{0}^{*}$ we obtain a complex

$$
W \otimes\left(\mathcal{R}_{0}^{*} \otimes \mathcal{R}_{2}\right)^{*} \otimes\left(\mathcal{R}_{0}^{*} \otimes \mathcal{R}_{1}\right) \stackrel{\operatorname{ad}(\rho)}{\rightarrow}\left(\mathcal{R}_{0}^{*} \otimes \mathcal{R}_{2}\right)^{*} \otimes\left(\mathcal{R}_{0}^{*} \otimes \mathcal{R}_{2}\right) \oplus\left(\mathcal{R}_{0}^{*} \otimes \mathcal{R}_{1}\right)^{*} \otimes\left(\mathcal{R}_{0}^{*} \otimes \mathcal{R}_{1}\right) \rightarrow \pi_{*} \mathcal{E}_{n d}\left(\mathcal{R}_{0}\right)
$$

which is acyclic in the middle and right term. Therefore, after descent to $\mathcal{M}_{m}^{s} \times \mathcal{M}_{m}^{s}$ it gives a complex $W \otimes F_{2}^{*} \otimes F_{1} \stackrel{\text { ad }(\rho)}{\rightarrow} F_{2}^{*} \otimes F_{2} \oplus F_{1}^{*} \otimes F_{1} \rightarrow \Delta_{*} \mathcal{A}_{\mathcal{M}}$ acyclic in the middle and in the right term.

We restrict the sheaf of Azumaya algebras $\mathcal{A}_{m}$ and $\mathcal{A}_{m}$-modules $\left(F_{1}, F_{2}\right)$ from $\mathcal{M}_{m}^{s}$ to $Y_{m}$ and denote the restrictions by the same symbols.

Theorem 5.7. Assume that we have a data (D.1), (D.2) satisfying the conditions (C.1)-(C.3). Assume that additional conditions (45) and (51) are satisfied. Then the Azumaya variety $\left(Y_{m}, \mathcal{A}_{m}\right)$ with the map $g_{m}: Y_{m} \rightarrow \mathbb{P}\left(V^{*}\right) \backslash \bar{Z}_{m}$, the pair of $\mathcal{A}_{m}$-modules $\left(F_{1}, F_{2}\right)$, and the canonical morphism $W \rightarrow \operatorname{Hom}\left(F_{1}, F_{2}\right)$ satisfy the conditions (C.4)-(C.6).

Proof: Condition (C.4) is proved in lemma 5.5, and condition (C.5) is proved in lemma 5.6. It remains to check condition (C.6). To this end denote $\widetilde{U}=\tilde{\mathbf{R}}_{m}^{1} \backslash \tilde{g}_{m}^{-1}\left(\bar{Z}_{m}\right)=\mathbf{R}_{m}^{1} \backslash q\left(\tilde{g}_{m}^{-1}\left(\bar{Z}_{m}\right)\right)$, so that $Y_{m}=\widetilde{U} / / \chi$, and recall that the composition

$$
\tilde{g}_{m}^{*} \mathcal{O}_{\mathbb{P}\left(V^{*}\right)}(-1) \rightarrow V^{*} \otimes \mathcal{O}_{U} \stackrel{\nu}{\longrightarrow} q_{*}\left(\left(E_{2}^{*}(1) \otimes \mathcal{R}_{2}^{*}\right) \otimes C\right)
$$

on $\widetilde{U}$ is zero. By adjunction we deduce that the composition

$$
q^{*} \tilde{g}_{m}^{*} \mathcal{O}_{\mathbb{P}\left(V^{*}\right)}(-1) \rightarrow V^{*} \otimes \mathcal{O}_{X \times U} \stackrel{\nu}{\longrightarrow}\left(E_{2}^{*}(1) \otimes \mathcal{R}_{2}^{*}\right) \otimes C
$$

on $X \times \widetilde{U}$ is zero. Tensoring by $q^{*} \tilde{g}_{m}^{*} \mathcal{O}_{\mathbb{P}\left(V^{*}\right)}(1)$ we deduce that $\nu$ takes the image $\lambda$ of the canonical element $\operatorname{id}_{V} \in V^{*} \otimes V=H^{0}\left(X, \mathcal{O}_{X}(1)\right) \otimes H^{0}\left(\mathbb{P}\left(V^{*}\right), \mathcal{O}_{\mathbb{P}\left(V^{*}\right)}(1)\right)$ in $H^{0}\left(X \times U, \mathcal{O}_{X}(1) \otimes \tilde{g}_{m}^{*} \mathcal{O}_{\mathbb{P}\left(V^{*}\right)}(1)\right)$ to $0 \in H^{0}\left(X \times U,\left(E_{2}^{*}(1) \otimes \mathcal{R}_{2}^{*} \otimes \tilde{g}_{m}^{*} \mathcal{O}_{\mathbb{P}\left(V^{*}\right)}(1)\right) \otimes C\right)$. This means that the composition of the left and bottom arrows in the following commutative diagram

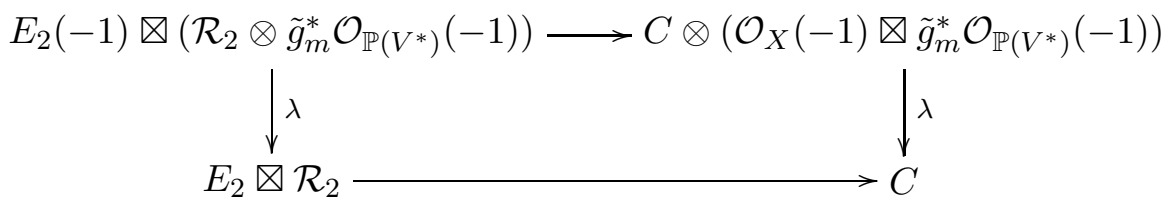

vanishes. But the upper arrow is an epimorphism. Hence the right arrow also vanishes. This means, that $C$ is supported scheme-theoretically on the zero locus of $\lambda$. But it is clear that after going down to the 
quotient $X \times Y_{m}=(X \times \widetilde{U}) / / \chi G$ the bundle $\mathcal{O}_{X}(1) \otimes \tilde{g}_{m}^{*} \mathcal{O}_{\mathbb{P}\left(V^{*}\right)}(1)$ descends to $\mathcal{O}_{X}(1) \otimes \mathcal{O}_{Y_{m}}(1)$ and its section $\lambda$ descends to the canonical section of $\mathcal{O}_{X}(1) \otimes \mathcal{O}_{Y_{m}}(1)$.

The problem thus reduces to the choice of $m$ such that conditions (C.7) and (C.8) are satisfied. I don't know how to make this choice in general. See however the following examples where the choices are made explicitly.

\section{EXAMPLES}

Examples in this section are ordered by the complexity of the variety $Y$. In examples 6.1 and 6.2 the variety $Y$ can be described explicitly and $\mathcal{A}_{Y}=\mathcal{O}_{Y}$. In examples $6.3,6.4$ and 6.5 we use the approach of section 5, consider the corresponding varieties $Y_{2}, Y_{3}$ and $Y_{4}$ respectively, and only after that give their description.

6.1. Grassmannian. Let $W=\mathrm{k}^{5}$ and

$$
X=\operatorname{Gr}(2, W)=\operatorname{Gr}(2,5), \quad\left(E_{1}, E_{2}\right)=\left(\mathcal{O}_{X}, \mathcal{U}_{X}^{*}\right),
$$

where $\mathcal{U}_{X} \subset W \otimes \mathcal{O}_{X}$ is the tautological rank 2 subbundle. Let $V=\Lambda^{2} W=\mathrm{k}^{10}$, and let $f: X \rightarrow \mathbb{P}(V)$ be the Plücker embedding. Take

$$
Y=\operatorname{Gr}\left(2, W^{*}\right)=\operatorname{Gr}(2,5), \quad \mathcal{A}_{Y}=\mathcal{O}_{Y}, \quad\left(F_{1}, F_{2}\right)=\left(\mathcal{U}_{Y}, \mathcal{O}_{Y}\right)
$$

where $\mathcal{U}_{Y} \subset W^{*} \otimes \mathcal{O}_{Y}$ is the tautological rank 2 subbundle. Let $g: Y \rightarrow \mathbb{P}\left(V^{*}\right)$ be the Plücker embedding (i.e. $\mathbf{Z}=\emptyset$ ). Then $\operatorname{Hom}\left(E_{1}, E_{2}\right)=W^{*}, \operatorname{Hom}\left(F_{1}, F_{2}\right)=W$, and we take $\phi=\mathrm{id}_{W}$.

It is easy to check that all conditions (C.1)-(C.8) are satisfied, so theorem 1.2 applies, and we have the following semiorthogonal decompositions (note that $\operatorname{codim}_{\mathbb{P}\left(V^{*}\right)}(Y)=3$, therefore for any admissible subspace $L \subset V^{*}$ of dimension $\operatorname{dim} L \leq 3$ we have $Y_{L}=\emptyset$ )

$$
\begin{aligned}
& \mathcal{D}^{b}(X)=\left\langle E_{1}(1), E_{2}(1), E_{1}(2), E_{2}(2), E_{1}(3), E_{2}(3), E_{1}(4), E_{2}(4), E_{1}(5), E_{2}(5)\right\rangle \\
& \mathcal{D}^{b}\left(X_{1}\right)=\left\langle E_{1}(1), E_{2}(1), E_{1}(2), E_{2}(2), E_{1}(3), E_{2}(3), E_{1}(4), E_{2}(4)\right\rangle \\
& \mathcal{D}^{b}\left(X_{2}\right)=\left\langle E_{1}(1), E_{2}(1), E_{1}(2), E_{2}(2), E_{1}(3), E_{2}(3)\right\rangle \\
& \mathcal{D}^{b}\left(X_{3}\right)=\left\langle E_{1}(1), E_{2}(1), E_{1}(2), E_{2}(2)\right\rangle \\
& \mathcal{D}^{b}\left(X_{4}\right)=\left\langle\mathcal{D}^{b}\left(Y_{4}\right), E_{1}(1), E_{2}(1)\right\rangle \\
& \mathcal{D}^{b}\left(X_{5}\right)=\mathcal{D}^{b}\left(Y_{5}\right)
\end{aligned}
$$

where $Y_{r}=Y \cap \mathbb{P}(L)$ is a linear section of $Y$ by an admissible subspace $L \subset V^{*}$ of dimension $r$, and $X_{r}=X \cap \mathbb{P}\left(L^{\perp}\right)$ is the corresponding orthogonal linear section of $X$ of codimension $r$. Note that $X_{1}$, $X_{2}$ and $X_{3}$ are always smooth, $X_{3}$ is the Fano threefold of index 2 and degree 5 ( $V_{5}$ threefold), $X_{4}$ is a del Pezzo surface of degree 5 (possibly singular), $Y_{4}$ is a zero-dimensional scheme of length $\operatorname{deg} Y=5$, and $X_{5}, Y_{5}$ are elliptic curves of degree 5 (possibly singular).

In particular, the results of Orlov [O1] about the derived category of the Fano threefold $V_{5}$ follow from theorem 1.2.

6.2. Orthogonal Grassmannian. Let $W=\mathrm{k}^{10}$ and

$$
X=\mathrm{OGr}_{+}(5, W), \quad\left(E_{1}, E_{2}\right)=\left(\mathcal{O}_{X}, \mathcal{U}_{X}^{*}\right),
$$

where $\mathrm{OGr}_{+}(5, W)$ is a connected component of the Grassmannian of 5-dimensional subspaces in $W$, isotropic with respect to a chosen nondegenerate quadratic form $\mathbf{q} \in S^{2} W^{*}$, and $\mathcal{U}_{X} \subset W \otimes \mathcal{O}_{X}$ is the tautological rank 5 subbundle. Let $V \cong \mathrm{k}^{16}$ be the corresponding half-spinor representation of the spin-group $\operatorname{Spin}(W)$ (see $[\mathrm{Ch}]$ ), and let $f: X \rightarrow \mathbb{P}(V)$ denote the canonical embedding. Take

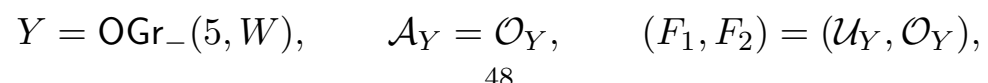


where $\mathcal{U}_{Y} \subset W \otimes \mathcal{O}_{Y}$ is the tautological rank 5 subbundle. We denote by $g: Y \rightarrow \mathbb{P}\left(V^{*}\right)$ the composition of the embedding $Y \rightarrow \mathbb{P}(V)$ and of the isomorphism $\mathbb{P}(V) \rightarrow \mathbb{P}\left(V^{*}\right)$ given by q. Then $\operatorname{Hom}\left(E_{1}, E_{2}\right) \cong W^{*}$, $\operatorname{Hom}\left(F_{1}, F_{2}\right) \cong W^{*}$, and we take $\phi=\mathbf{q}^{-1}$.

It is easy to check that all conditions (C.1)-(C.8) are satisfied, so theorem 1.2 applies, and we have the following semiorthogonal decompositions (note that $\operatorname{codim}_{\mathbb{P}\left(V^{*}\right)}(Y)=5$, therefore for any admissible subspace $L \subset V^{*}$ of dimension $\operatorname{dim} L \leq 5$ we have $Y_{L}=\emptyset$ )

$$
\begin{aligned}
& \mathcal{D}^{b}(X)=\left\langle E_{1}(1), E_{2}(1), E_{1}(2), E_{2}(2), E_{1}(3), E_{2}(3), E_{1}(4), E_{2}(4), E_{1}(5), E_{2}(5), E_{1}(6), E_{2}(6), E_{1}(7), E_{2}(7), E_{1}(8), E_{2}(8)\right\rangle \\
& \mathcal{D}^{b}\left(X_{1}\right)=\left\langle E_{1}(1), E_{2}(1), E_{1}(2), E_{2}(2), E_{1}(3), E_{2}(3), E_{1}(4), E_{2}(4), E_{1}(5), E_{2}(5), E_{1}(6), E_{2}(6), E_{1}(7), E_{2}(7)\right\rangle \\
& \mathcal{D}^{b}\left(X_{2}\right)=\left\langle E_{1}(1), E_{2}(1), E_{1}(2), E_{2}(2), E_{1}(3), E_{2}(3), E_{1}(4), E_{2}(4), E_{1}(5), E_{2}(5), E_{1}(6), E_{2}(6)\right\rangle \\
& \mathcal{D}^{b}\left(X_{3}\right)=\left\langle E_{1}(1), E_{2}(1), E_{1}(2), E_{2}(2), E_{1}(3), E_{2}(3), E_{1}(4), E_{2}(4), E_{1}(5), E_{2}(5)\right\rangle \\
& \mathcal{D}^{b}\left(X_{4}\right)=\left\langle E_{1}(1), E_{2}(1), E_{1}(2), E_{2}(2), E_{1}(3), E_{2}(3), E_{1}(4), E_{2}(4)\right\rangle \\
& \mathcal{D}^{b}\left(X_{5}\right)=\left\langle E_{1}(1), E_{2}(1), E_{1}(2), E_{2}(2), E_{1}(3), E_{2}(3)\right\rangle \\
& \mathcal{D}^{b}\left(X_{6}\right)=\left\langle\mathcal{D}^{b}\left(Y_{6}\right), E_{1}(1), E_{2}(1), E_{1}(2), E_{2}(2)\right\rangle \\
& \mathcal{D}^{b}\left(X_{7}\right)=\left\langle\mathcal{D}^{b}\left(Y_{7}\right), E_{1}(1), E_{2}(1)\right\rangle \\
& \mathcal{D}^{b}\left(X_{8}\right)=\mathcal{D}^{b}\left(Y_{8}\right)
\end{aligned}
$$

where $Y_{r}=Y \cap \mathbb{P}(L)$ is a linear section of $Y$ by an admissible subspace $L \subset V^{*}$ of dimension $r$, and $X_{r}=X \cap \mathbb{P}\left(L^{\perp}\right)$ is the corresponding orthogonal linear section of $X$ of codimension $r$. Note that $X_{1}$, $X_{2}, X_{3}, X_{4}$ and $X_{5}$ are always smooth, $X_{6}$ is a Fano fourfold of index 2 (possibly singular), $Y_{6}$ is a zero-dimensional scheme of length $\operatorname{deg} Y=12, X_{7}$ is a Fano threefold $V_{12}$ (possibly singular), $Y_{7}$ is a curve of genus 7 (possibly singular), and $X_{8}, Y_{8}$ are $K 3$-surfaces of degree 12 (possibly singular).

In particular, the results of $[\mathrm{K}]$ about the derived category of the Fano threefold $V_{12}$ follow from theorem 1.2. Indeed when $X_{7}$ is a smooth Fano threefold $V_{16}$ then $Y_{7}$ is a smooth curve of genus 7 , and we obtain a decomposition

$$
\mathcal{D}^{b}\left(V_{12}\right)=\left\langle\mathcal{D}^{b}\left(C_{7}\right), \mathcal{O}, \mathcal{U}^{*}\right\rangle
$$

where $C_{7}$ is a curve of genus 7 .

6.3. Lagrangian Grassmannian. Let $W=\mathrm{k}^{6}$ and

$$
X=\operatorname{LGr}(3, W), \quad\left(E_{1}, E_{2}\right)=\left(\mathcal{O}_{X}, \mathcal{U}_{X}^{*}\right),
$$

where $\operatorname{LGr}(3, W)$ is the Grassmannian of 3-dimensional subspaces in $W$, Lagrangian with respect to a chosen symplectic form $\sigma \in \Lambda^{2} W^{*}$, and $\mathcal{U}_{X} \subset W \otimes \mathcal{O}_{X}$ is the tautological rank 3 subbundle. The natural representation of the group $\mathrm{Sp}(W)$ in the space $\Lambda^{3} W$ decomposes into the direct sum of representations

$$
\Lambda^{3} W=W \oplus V .
$$

The Plücker embedding $\operatorname{Gr}(3, W) \subset \mathbb{P}\left(\Lambda^{3} W\right)$ restricts to an embedding $f: X \rightarrow \mathbb{P}(V)$. Now we apply the construction of section 5 and take $Y=Y_{2}, \mathcal{A}_{Y}=\mathcal{A}_{2}, \mathbf{Z}=\bar{Z}_{2}, g=g_{2}$, the corresponding universal family $\left(F_{1}, F_{2}\right)$, and the map $\phi$ induced by the Q-representation structure on $\left(F_{1}, F_{2}\right)$.

It is shown in Appendix A that all conditions (C.1)-(C.8) are satisfied, and in fact

$$
\mathbf{Z}=\mu\left(\operatorname{Gr}\left(3, W^{*}\right)\right)=\mu(\operatorname{Gr}(3,6)),
$$

where $\mu: \mathbb{P}\left(\Lambda^{3} W^{*}\right)-->\mathbb{P}\left(V^{*}\right)$ is the linear projection from $\mathbb{P}\left(\sigma \wedge W^{*}\right)$, and

$$
Y=X^{\vee} \backslash \mathbf{Z}
$$

where $X^{\vee}$ is the projectively dual variety of $X$, which is a quartic hypersurface, singular along $\mathbf{Z} \subset \mathbb{P}\left(V^{*}\right)$ (see $[\mathrm{Ho}]$ ). Thus we have the following semiorthogonal decompositions (note that $\operatorname{codim}_{\mathbb{P}\left(V^{*}\right)}(Y)=1$, 
therefore for any admissible subspace $L \subset V^{*}$ of dimension $\operatorname{dim} L \leq 1$ we have $Y_{L}=\emptyset$ )

$$
\begin{aligned}
& \mathcal{D}^{b}(X)=\left\langle E_{1}(1), E_{2}(1), E_{1}(2), E_{2}(2), E_{1}(3), E_{2}(3), E_{1}(4), E_{2}(4)\right\rangle \\
& \mathcal{D}^{b}\left(X_{1}\right)=\left\langle E_{1}(1), E_{2}(1), E_{1}(2), E_{2}(2), E_{1}(3), E_{2}(3)\right\rangle \\
& \mathcal{D}^{b}\left(X_{2}\right)=\left\langle\mathcal{D}^{b}\left(Y_{2}\right), E_{1}(1), E_{2}(1), E_{1}(2), E_{2}(2)\right\rangle \\
& \mathcal{D}^{b}\left(X_{3}\right)=\left\langle\mathcal{D}^{b}\left(Y_{3}, \mathcal{A}_{Y}\right), E_{1}(1), E_{2}(1)\right\rangle \\
& \mathcal{D}^{b}\left(X_{4}\right)=\mathcal{D}^{b}\left(Y_{4}, \mathcal{A}_{Y}\right)
\end{aligned}
$$

where $Y_{r}=Y \cap \mathbb{P}(L)$ is a linear section of $Y$ by an admissible subspace $L \subset V^{*}$ of dimension $r$, and $X_{r}=X \cap \mathbb{P}\left(L^{\perp}\right)$ is the corresponding orthogonal linear section of $X$ of codimension $r$. Note that $X_{1}$ is always smooth, $X_{2}$ is a Fano fourfold of index 2 (possibly singular), $Y_{2}$ is a zero-dimensional scheme of length $\operatorname{deg} Y=4, X_{3}$ is a Fano threefold $V_{16}$ (possibly singular), $Y_{3}$ is a plane quartic (possibly singular), and $X_{4}, Y_{4}$ are $K 3$-surfaces of degree 16 and 4 respectively (possibly singular).

In particular, the results of [S1, S2] about the derived category of the Lagrangian Grassmannian $X$ and of its hyperplane section $X_{1}$ follow from theorem 1.2. Moreover, when $X_{3}$ is a smooth Fano threefold $V_{16}$ then $Y_{3}$ is a smooth curve of genus 3 , hence $\mathcal{D}^{b}\left(Y_{3}, \mathcal{A}_{Y}\right) \cong \mathcal{D}^{b}\left(Y_{3}\right)$ (the Brauer group of a smooth curve is trivial), and we obtain a decomposition

$$
\mathcal{D}^{b}\left(V_{16}\right)=\left\langle\mathcal{D}^{b}\left(C_{3}\right), \mathcal{O}, \mathcal{U}^{*}\right\rangle
$$

where $C_{3}$ is a curve of genus 3 .

6.4. $G_{2}$ Grassmannian. Let $W=\mathrm{k}^{7}$ and

$$
X=\mathrm{G}_{2} \operatorname{Gr}(2, W), \quad\left(E_{1}, E_{2}\right)=\left(\mathcal{O}_{X}, \mathcal{U}_{X}^{*}\right),
$$

where $\mathrm{G}_{2} \mathrm{Gr}(3, W)$ is the Grassmannian of the simple Lie group $G_{2}$, realized as the zero locus of the section $s_{\lambda} \in H^{0}\left(\operatorname{Gr}(2, W), \mathcal{U}^{\perp}(1)\right) \cong \Lambda^{3} W^{*}$, corresponding to a unique $G_{2}$-invariant 3-form $\lambda$ on the tautological $G_{2}$-representation $W, \mathcal{U} \subset W \otimes \mathcal{O}_{\mathrm{Gr}(2, W)}$ is the tautological rank 2 subbundle, and $\mathcal{U}_{X}$ is the restriction of $\mathcal{U}$ to $X$. The natural representation of the group $G_{2}$ in the space $\Lambda^{2} W$ decomposes into the direct sum of representations

$$
\Lambda^{2} W=W^{*} \oplus V
$$

(the projection $\Lambda^{2} W \rightarrow W^{*}$ is given by $\lambda$ ). The Plücker embedding $\operatorname{Gr}(2, W) \subset \mathbb{P}\left(\Lambda^{2} W\right)$ restricts to an embedding $f: X \rightarrow \mathbb{P}(V)$. Now we apply the construction of section 5 and take $Y=Y_{3}$, $\mathcal{A}_{Y}=\mathcal{A}_{3}, \mathbf{Z}=\bar{Z}_{3}, g=g_{3}$, the corresponding universal family $\left(F_{1}, F_{2}\right)$, and the map $\phi$ induced by the Q-representation structure on $\left(F_{1}, F_{2}\right)$.

It is shown in Appendix B that all conditions (C.1)-(C.8) are satisfied, and in fact

$$
\mathbf{Z}=\mu\left(\operatorname{Gr}\left(2, W^{*}\right)\right)=\mu(\operatorname{Gr}(2,7)),
$$

where $\mu: \mathbb{P}\left(\Lambda^{2} W^{*}\right)-->\mathbb{P}\left(V^{*}\right)$ is the linear projection from $\mathbb{P}(\lambda(W))$, and

$$
g: Y \stackrel{2: 1}{\longrightarrow} \mathbb{P}\left(V^{*}\right) \backslash \mathbf{Z} \quad \text { is the double covering ramified at } \quad X^{\vee} \backslash \mathbf{Z} \subset \mathbb{P}\left(V^{*}\right) \backslash \mathbf{Z},
$$

where $X^{\vee}$ is the projectively dual variety of $X$ which is a sextic hypersurface, singular along $\mathbf{Z} \subset \mathbb{P}\left(V^{*}\right)$ (see $[\mathrm{Ho}]$ ). Thus we have the following semiorthogonal decompositions

$$
\begin{aligned}
& \mathcal{D}^{b}(X)=\left\langle E_{1}(1), E_{2}(1), E_{1}(2), E_{2}(2), E_{1}(3), E_{2}(3)\right\rangle \\
& \mathcal{D}^{b}\left(X_{1}\right)=\left\langle\mathcal{D}^{b}\left(Y_{1}\right), E_{1}(1), E_{2}(1), E_{1}(2), E_{2}(2)\right\rangle \\
& \mathcal{D}^{b}\left(X_{2}\right)=\left\langle\mathcal{D}^{b}\left(Y_{2}, \mathcal{A}_{Y}\right), E_{1}(1), E_{2}(1)\right\rangle \\
& \mathcal{D}^{b}\left(X_{3}\right)=\mathcal{D}^{b}\left(Y_{3}, \mathcal{A}_{Y}\right)
\end{aligned}
$$

where $Y_{r}=Y \cap \mathbb{P}(L)$ is a linear section of $Y$ by an admissible subspace $L \subset V^{*}$ of dimension $r$, and $X_{r}=X \cap \mathbb{P}\left(L^{\perp}\right)$ is the corresponding orthogonal linear section of $X$ of codimension $r$. Note that $X_{1}$ is 
a Fano fourfold of index 2 (possibly singular), $Y_{1}$ is a zero-dimensional scheme of length $\operatorname{deg} Y=2, X_{2}$ is a Fano threefold $V_{18}$ (possibly singular), $Y_{2}$ is a curve of genus 2 (possibly singular), and $X_{3}, Y_{3}$ are $K 3$-surfaces of degree 18 and 2 respectively (possibly singular).

In particular, the results of [Ra] about the derived category of the $G_{2}$-Grassmannian $X$ follow from theorem 1.2. Moreover, when $X_{2}$ is a smooth Fano threefold $V_{18}$ then $Y_{2}$ is a smooth curve of genus 2 (by projective duality), hence $\mathcal{D}^{b}\left(Y_{2}, \mathcal{A}_{Y}\right) \cong \mathcal{D}^{b}\left(Y_{2}\right)$ (the Brauer group of a smooth curve is trivial), and we obtain a decomposition

$$
\mathcal{D}^{b}\left(V_{18}\right)=\left\langle\mathcal{D}^{b}\left(C_{2}\right), \mathcal{O}, \mathcal{U}^{*}\right\rangle
$$

where $C_{2}$ is a curve of genus 2 .

6.5. Intersection of quadrics. Let $W=\mathrm{k}^{6}$ and

$$
X=\mathbb{P}(W)=\mathbb{P}^{5}, \quad\left(E_{1}, E_{2}\right)=\left(\mathcal{O}_{X}, \mathcal{O}_{X}(1)\right) .
$$

Let $V=S^{2} W=\mathrm{k}^{21}$ and let $f: X \rightarrow \mathbb{P}(V)$ be the double Veronese embedding.

Now we apply the construction of section 5 and take $Y=Y_{4}, \mathcal{A}_{Y}=\mathcal{A}_{4}, \mathbf{Z}=\bar{Z}_{4}, g=g_{4}$, the corresponding universal family $\left(F_{1}, F_{2}\right)$, and the map $\phi$ induced by the Q-representation structure on $\left(F_{1}, F_{2}\right)$.

It is shown in Appendix $\mathbf{C}$ that all conditions (C.1)-(C.8) are satisfied, and in fact $\mathbf{Z} \subset \mathbb{P}\left(S^{2} W^{*}\right)$ is the locus of rank 4 (corank 2) quadratic forms and

$$
g: Y \stackrel{2: 1}{\longrightarrow} \mathbb{P}\left(V^{*}\right) \backslash \mathbf{Z} \quad \text { is the double covering ramified at } \quad X^{\vee} \backslash \mathbf{Z} \subset \mathbb{P}\left(V^{*}\right) \backslash \mathbf{Z},
$$

where $X^{\vee}$ is the projectively dual variety of $X$ which is the determinantal sextic hypersurface (the locus of degenerate quadratic forms), singular along $\mathbf{Z} \subset \mathbb{P}\left(V^{*}\right)$. Thus we have the following semiorthogonal decompositions

$$
\begin{aligned}
& \mathcal{D}^{b}(X)=\left\langle\mathcal{O}_{X}(1), \mathcal{O}_{X}(2), \mathcal{O}_{X}(3), \mathcal{O}_{X}(4), \mathcal{O}_{X}(5), \mathcal{O}_{X}(6)\right\rangle \\
& \mathcal{D}^{b}\left(X_{1}\right)=\left\langle\mathcal{D}^{b}\left(Y_{1}\right), \mathcal{O}_{X}(1), \mathcal{O}_{X}(2), \mathcal{O}_{X}(3), \mathcal{O}_{X}(4)\right\rangle \\
& \mathcal{D}^{b}\left(X_{2}\right)=\left\langle\mathcal{D}^{b}\left(Y_{2}, \mathcal{A}_{Y}\right), \mathcal{O}_{X}(1), \mathcal{O}_{X}(2)\right\rangle \\
& \mathcal{D}^{b}\left(X_{3}\right)=\mathcal{D}^{b}\left(Y_{3}, \mathcal{A}_{Y}\right)
\end{aligned}
$$

where $Y_{r}=Y \cap \mathbb{P}(L)$ is a linear section of $Y$ by an admissible subspace $L \subset V^{*}$ of dimension $r$, and $X_{r}=X \cap \mathbb{P}\left(L^{\perp}\right)$ is the corresponding orthogonal linear section of $X$ of codimension $r$. Note that $X_{1}$ is a four-dimensional quadric (possibly singular), $Y_{1}$ is a zero-dimensional scheme of length $2, X_{2}$ is a Fano threefold of index 2 and degree 4 (an intersection of two four-dimensional quadrics) (possibly singular), $Y_{2}$ is a curve of genus 2 (possibly singular), and $X_{3}, Y_{3}$ are $K 3$-surfaces of degree 8 and 2 respectively (possibly singular).

In particular, the results of [BO1] about the derived category of an intersection of two four-dimensional quadrics follow from theorem 1.2.

It is also worth to note that the sheaf of Azumaya algebras $\mathcal{A}_{Y}$ on $Y$ can be constructed in quite another way. More precisely, one can check that the sheaf of algebras $g_{*} \mathcal{A}_{Y}$ is isomorphic to the even part of the universal Clifford algebra (see the introduction).

\section{Appendix A. Lagrangian Grassmannian}

In this appendix we show that a quartic hypersurface in $\mathbb{P}^{13}$ is homologically projectively dual to the Lagrangian Grassmannian LGr $(3,6)$.

Recall the notation. Let $W=\mathrm{k}^{6}$ and

$$
X=\operatorname{LGr}(3, W), \quad\left(E_{1}, E_{2}\right)=\left(\mathcal{O}_{X}, \mathcal{U}_{X}^{*}\right),
$$


where $\operatorname{LGr}(3, W)$ is the Grassmannian of 3-dimensional subspaces in $W$, Lagrangian with respect to a chosen symplectic form $\sigma \in \Lambda^{2} W^{*}$, and $\mathcal{U}_{X} \subset W \otimes \mathcal{O}_{X}$ is the tautological rank 3 subbundle. The natural representation of the group $\mathrm{Sp}(W)$ in the space $\Lambda^{3} W$ decomposes into the direct sum of representations

$$
\Lambda^{3} W=W \oplus V .
$$

The Plücker embedding $\operatorname{Gr}(3, W) \subset \mathbb{P}\left(\Lambda^{3} W\right)$ restricts to an embedding $f: X \rightarrow \mathbb{P}(V)$.

Lemma A.1. We have $\operatorname{dim} X=6, \omega_{X} \cong \mathcal{O}_{X}(-4)$. Moreover, conditions (C.1)-(C.3) as well as the additional conditions (45) and (51) are satisfied for $X$.

Proof: Note that $X \subset \operatorname{Gr}(3, W)$ is a zero locus of a regular section $s_{\sigma} \in H^{0}\left(\operatorname{Gr}(3, W), \Lambda^{2} \mathcal{U}^{*}\right)$. Hence

$$
\begin{aligned}
& \operatorname{dim} X=\operatorname{dim} \operatorname{Gr}(3, W)-\operatorname{rank}\left(\Lambda^{2} \mathcal{U}^{*}\right)=9-3=6, \\
& \omega_{X} \cong \omega_{\operatorname{Gr}(3, W) \mid X} \otimes \operatorname{det}\left(\Lambda^{2} \mathcal{U}^{*}\right) \cong \mathcal{O}_{X}(-6) \otimes \mathcal{O}_{X}(2) \cong \mathcal{O}_{X}(-4) .
\end{aligned}
$$

In particular, (C.2) is true and $i_{X}=4$. Further, using the Koszul resolution $\Lambda^{\bullet}\left(\Lambda^{2} \mathcal{U}\right) \cong \mathcal{O}_{X}$ of $X$ in $\operatorname{Gr}(3, W)$ it is easy to check that $H^{0}\left(X, \mathcal{O}_{X}(1)\right) \cong V^{*}$, hence $f(X)$ is not contained in a hyperplane, i.e. (C.1) is true. Using again the Koszul resolution we compute $\operatorname{Hom}\left(E_{s}(k), E_{t}(l)\right)$ via Borel-Bott-Weil theorem and check that (C.3) and (45) is true. Finally, we note that $\mathbf{R}_{1}=\operatorname{Hom}\left(\mathrm{k}^{3}, W^{*}\right)$ and $\mathbf{R}_{1}^{\circ} \subset \mathbf{R}_{1}$ corresponds to the subset of embeddings $k^{3} \subset W^{*}$. Therefore, $\operatorname{codim}_{\mathbf{R}_{1}}\left(\mathbf{R}_{1} \backslash \mathbf{R}_{1}^{\circ}\right)=4$ and (51) is satisfied by lemma 5.3 .

Now we are going to apply the construction of section 5 and describe the space $Y=Y_{2}$ explicitly. The question turns out to be rather cumbersome. However, it seems easier to describe instead $\mathbb{P}_{Y_{2}, \mathcal{A}_{2}}\left(F_{2}^{*}\right)$, the moduli space of quotient $\mathcal{A}_{2}$-modules of rank 1 . Indeed, the fiber of $\mathbb{P}_{Y_{2}, \mathcal{A}_{2}}\left(F_{2}^{*}\right)$ at the point of $Y_{2}$ corresponding to a $(6,2)$-dimensional representation $\rho=\left(R_{1}, R_{2}\right)$ of $\mathrm{Q}$ coincides with $\mathbb{P}\left(R_{2}\right)$. We show that any line $R_{2}^{\prime} \subset R_{2}$ can be extended in a unique way to a $(1,1)$-dimensional subrepresentation $\rho^{\prime}=\left(R_{1}^{\prime}, R_{2}^{\prime}\right)$. The moduli space of $(1,1)$-dimensional representations of $\mathrm{Q}$ is $\mathbb{P}(W)$. We claim that defined in this way map $\mathbb{P}_{Y_{2}, \mathcal{A}_{2}}\left(F_{2}^{*}\right) \rightarrow \mathbb{P}(W)$ identifies $\mathbb{P}_{Y_{2}, \mathcal{A}_{2}}\left(F_{2}^{*}\right)$ with an open subset in a $\mathbb{P}^{8}$-bundle over $\mathbb{P}(W)$, explicitly $\widetilde{Y}=\mathbb{P}_{\mathbb{P}(W)}\left(\Omega_{\mathbb{P}(W)}^{3}(3) / \mathcal{O}_{\mathbb{P}(W)}(-1)\right)$. To prove this we go the other way round. We consider the space $\tilde{Y}$, and identify its open subset with $\mathbb{P}_{Y_{2}, \mathcal{A}_{2}}\left(F_{2}^{*}\right)$.

We start with several useful lemmas.

Lemma A.2. We have $\bar{Y}_{1}=\bar{Z}_{1}=\mu\left(\operatorname{Gr}\left(3, W^{*}\right)\right)$, where $\mu: \mathbb{P}\left(\Lambda^{3} W^{*}\right)--\rightarrow \mathbb{P}\left(V^{*}\right)$ is the linear projection from $\mathbb{P}\left(\sigma \wedge W^{*}\right) \subset \mathbb{P}\left(\Lambda^{3} W^{*}\right)$.

Proof: If $m=1$ then $\left(d_{1}, d_{2}\right)=(3,1)$. A $(3,1)$-dimensional representation $\rho: W \otimes R_{1} \rightarrow R_{2}$ is $\chi$ semistable iff the corresponding map $R_{1} \otimes R_{2}^{*} \rightarrow W^{*}$ is an embedding. Thus $\mathcal{M}_{1}^{s s}$ gets identified with $\operatorname{Gr}\left(3, W^{*}\right)$. Moreover, it is easy to see that in this case every hyperplane section of $X$ is irreducible, and we have $\tilde{\mathbf{R}}_{1}^{1}=\mathbf{R}_{1}^{1}=\mathbf{R}_{1}^{\circ}=\mathbf{R}_{1}^{s s}$ and the lemma follows.

Consider a product $X \times \mathbb{P}(W)$ and a subscheme $\mathbb{P}_{X}\left(\mathcal{U}_{X}\right) \subset X \times \mathbb{P}(W)$.

Lemma A.3. The following sequences on $X \times \mathbb{P}(W)$ are exact

$$
\begin{aligned}
0 & \rightarrow \mathcal{O}_{X}(-1) \otimes \mathcal{O}_{\mathbb{P}(W)}(-3) \rightarrow \Lambda^{2} \mathcal{U}_{X} \otimes \mathcal{O}_{\mathbb{P}(W)}(-2) \rightarrow \mathcal{U}_{X} \otimes \mathcal{O}_{\mathbb{P}(W)}(-1) \rightarrow \mathcal{O}_{X} \otimes \mathcal{O}_{\mathbb{P}(W)} \rightarrow \mathcal{O}_{\mathbb{P}_{X}\left(\mathcal{U}_{X}\right)} \rightarrow 0, \\
0 & \rightarrow \mathcal{O}_{X}(-1) \otimes \mathcal{O}_{\mathbb{P}(W)}(-3) \rightarrow \mathcal{U}_{X}^{*}(-1) \otimes \mathcal{O}_{\mathbb{P}(W)}(-2) \rightarrow \mathcal{O}_{X} \otimes \Omega_{\mathbb{P}(W)} \rightarrow \mathcal{U}_{X}^{*} \otimes \mathcal{O}_{\mathbb{P}(W)}(-1) \rightarrow \mathcal{O}_{\mathbb{P}_{X}\left(\mathcal{U}_{X}\right)} \rightarrow 0 .
\end{aligned}
$$

Proof: The first sequence is just the Koszul resolution of $\mathbb{P}_{X}\left(\mathcal{U}_{X}\right) \subset X \times \mathbb{P}(W)$ (note that $\mathcal{U}_{X}^{\perp} \cong \mathcal{U}_{X}$, the isomorphism is given by $\sigma$ ). Further, it follows from exact sequences

$$
\begin{aligned}
0 \longrightarrow \mathcal{U}_{X} \otimes \mathcal{O}_{\mathbb{P}(W)}(-1) \longrightarrow W^{*} \otimes \mathcal{O}_{X} \otimes \mathcal{O}_{\mathbb{P}(W)}(-1) \longrightarrow \mathcal{U}_{X}^{*} \otimes \mathcal{O}_{\mathbb{P}(W)}(-1) \longrightarrow 0 \\
0 \longrightarrow \mathcal{O}_{X} \otimes \Omega_{\mathbb{P}(W)} \longrightarrow W^{*} \otimes \mathcal{O}_{X} \otimes \mathcal{O}_{\mathbb{P}(W)}(-1) \longrightarrow \mathcal{O}_{X} \otimes \mathcal{O}_{\mathbb{P}(W)} \longrightarrow
\end{aligned}
$$


that the kernel and the cokernel of the composition maps $\mathcal{U}_{X} \otimes \mathcal{O}_{\mathbb{P}(W)}(-1) \rightarrow \mathcal{O}_{X} \otimes \mathcal{O}_{\mathbb{P}(W)}$ and $\mathcal{O}_{X} \otimes$ $\Omega_{\mathbb{P}(W)} \rightarrow \mathcal{U}_{X}^{*} \otimes \mathcal{O}_{\mathbb{P}(W)}(-1)$ coincide. Taking also into account that $\Lambda^{2} \mathcal{U}_{X} \cong \mathcal{U}_{X}^{*}(-1)$ we obtain the second sequence from the first one.

Consider the truncated first sequence of lemma A.3 as a resolution of the sheaf of ideals $J_{\mathbb{P}_{X}}\left(\mathcal{U}_{X}\right)$. Tensoring it with $\mathcal{O}_{X}(1)$ and pushing forward to $\mathbb{P}(W)$ we obtain exact sequence

$$
0 \rightarrow \mathcal{O}_{\mathbb{P}(W)}(-3) \rightarrow W^{*} \otimes \mathcal{O}_{\mathbb{P}(W)}(-2) \rightarrow\left(\Lambda^{2} W^{*} / \mathrm{k} \sigma\right) \otimes \mathcal{O}_{\mathbb{P}(W)}(-1) \rightarrow p_{2_{*}}\left(J_{\mathbb{P}_{X}}\left(\mathcal{U}_{X}\right) \otimes \mathcal{O}_{X}(1)\right) \rightarrow 0,
$$

where $p_{2}: X \times \mathbb{P}(W) \rightarrow \mathbb{P}(W)$ is the projection. Taking into account exact sequence

$$
0 \rightarrow \mathcal{O}_{\mathbb{P}(W)}(-3) \rightarrow W^{*} \otimes \mathcal{O}_{\mathbb{P}(W)}(-2) \rightarrow \Lambda^{2} W^{*} \otimes \mathcal{O}_{\mathbb{P}(W)}(-1) \rightarrow \Omega_{\mathbb{P}(W)}^{3}(3) \rightarrow 0
$$

we can rewrite it as

$$
0 \rightarrow \mathcal{O}_{\mathbb{P}(W)}(-1) \stackrel{\sigma}{\longrightarrow} \Omega_{\mathbb{P}(W)}^{3}(3) \rightarrow p_{2 *}\left(J_{\mathbb{P}_{X}\left(\mathcal{U}_{X}\right)} \otimes \mathcal{O}_{X}(1)\right) \rightarrow 0 .
$$

Lemma A.4. Morphism $\mathcal{O}_{\mathbb{P}(W)}(-1) \stackrel{\sigma}{\longrightarrow} \Omega_{\mathbb{P}(W)}^{3}(3)$ is an embedding of vector bundles on $\mathbb{P}(W)$.

Proof: This morphism is $\mathrm{Sp}(W)$-equivariant and $\mathrm{Sp}(W)$ acts transitively on $\mathbb{P}(W)$.

Consider the projectivization of the quotient bundle

$$
\tilde{Y}=\mathbb{P}_{\mathbb{P}(W)}\left(\Omega_{\mathbb{P}(W)}^{3}(3) / \mathcal{O}_{\mathbb{P}(W)}(-1)\right)=\mathbb{P}_{\mathbb{P}(W)}\left(p_{2 *}\left(J_{\mathbb{P}_{X}\left(\mathcal{U}_{X}\right)} \otimes \mathcal{O}_{X}(1)\right)\right) .
$$

The natural embedding $p_{2 *}\left(J_{\mathbb{P}_{X}\left(\mathcal{U}_{X}\right)} \otimes \mathcal{O}_{X}(1)\right) \rightarrow H^{0}\left(X, \mathcal{O}_{X}(1)\right) \otimes \mathcal{O}_{\mathbb{P}(W)}=V^{*} \otimes \mathcal{O}_{\mathbb{P}(W)}$ induces a morphism $\tilde{Y} \stackrel{\tilde{g}}{\longrightarrow} \mathbb{P}\left(V^{*}\right)$. Let $\pi: \tilde{Y} \rightarrow \mathbb{P}(W)$ denote the canonical projection. Denote also

$$
\mathcal{O}_{\widetilde{Y}}(H):=\tilde{g}^{*} \mathcal{O}_{\mathbb{P}(V)}(1), \quad \tilde{g}: \widetilde{Y} \rightarrow \mathbb{P}\left(V^{*}\right), \quad \text { and } \quad \mathcal{O}_{\widetilde{Y}}(h):=\pi^{*} \mathcal{O}_{\mathbb{P}(W)}(1), \quad \pi: \tilde{Y} \rightarrow \mathbb{P}(W) .
$$

Consider the product $X \times \tilde{Y}$, the projection $\operatorname{id}_{X} \times \pi: X \times \tilde{Y} \rightarrow X \times \mathbb{P}(W)$, the subschemes

$$
\Sigma=\left(\operatorname{id}_{X} \times \pi\right)^{-1}\left(\mathbb{P}_{X}\left(\mathcal{U}_{X}\right)\right), \quad \tilde{Q}=Q(X, \widetilde{Y})=(X \times \widetilde{Y}) \times_{\mathbb{P}(V) \times \mathbb{P}\left(V^{*}\right)} Q,
$$

and the equation $s_{\tilde{Q}} \in H^{0}\left(X \times \tilde{Y}, \mathcal{O}_{X}(1) \otimes \mathcal{O}_{\tilde{Y}}(H)\right)=H^{0}\left(X \times \tilde{Y},(f \times \tilde{g})^{*} \mathcal{O}_{\mathbb{P}(V) \times \mathbb{P}\left(V^{*}\right)}(1,1)\right) \cong V^{*} \otimes V$ of $\tilde{Q}$ in $X \times \tilde{Y}$.

Lemma A.5. The section $s_{\tilde{Q}}$ vanishes on the scheme $\Sigma$.

Proof: We have on $\widetilde{Y}$ a canonical embedding

$$
\mathcal{O}_{\widetilde{Y}}(-H) \rightarrow \pi^{*} p_{2 *}\left(J_{\mathbb{P}_{X}}\left(\mathcal{U}_{X}\right) \otimes \mathcal{O}_{X}(1)\right) \cong p_{2 *}\left(J_{\Sigma} \otimes \mathcal{O}_{X}(1)\right)
$$

Tensoring it by $\mathcal{O}_{\widetilde{Y}}(H)$ we obtain a canonical morphism $\mathcal{O}_{X \times \widetilde{Y}} \rightarrow J_{\Sigma} \otimes\left(\mathcal{O}_{X}(1) \otimes \mathcal{O}_{\widetilde{Y}}(H)\right)$. It is clear that the composition of this morphism with embedding $J_{\Sigma} \otimes\left(\mathcal{O}_{X}(1) \otimes \mathcal{O}_{\widetilde{Y}}(H)\right) \rightarrow \mathcal{O}_{X}(1) \otimes \mathcal{O}_{\widetilde{Y}}(H)$ coincides with $s_{\tilde{Q}}$.

Consider the pullback of the second exact sequence of lemma A.3 to $X \times \tilde{Y}$. Consider the cone $G^{\prime}$ of its first morphism twisted by $\mathcal{O}_{X}(1) \otimes \mathcal{O}_{\widetilde{Y}}(H)$, and the cone $G^{\prime \prime}$ of its third morphism as objects of $\mathcal{D}^{b}(X \times \tilde{Y})$, so that we have exact triangles

$$
\begin{gathered}
E_{1} \otimes \mathcal{O}_{\widetilde{Y}}(H-3 h) \stackrel{\tilde{e}^{\prime}}{\longrightarrow} E_{2} \otimes \mathcal{O}_{\widetilde{Y}}(H-2 h) \longrightarrow G^{\prime} \\
E_{1} \otimes \pi^{*} \Omega_{\mathbb{P}(W)} \stackrel{\tilde{e}^{\prime \prime}}{\longrightarrow} E_{2} \otimes \mathcal{O}_{\widetilde{Y}}(-h) \longrightarrow G^{\prime \prime}
\end{gathered}
$$

Then the pullback of the second exact sequence of lemma A.3 can be rewritten as exact triangle

$$
G^{\prime} \otimes\left(\mathcal{O}_{X}(-1) \otimes \mathcal{O}_{\widetilde{Y}}(-H)\right)[1] \rightarrow G^{\prime \prime} \rightarrow \mathcal{O}_{\Sigma}
$$


It is clear that morphisms $\tilde{e}^{\prime}$ and $\tilde{e}^{\prime \prime}$ determine a structure of Q-representations on families $\left(\mathcal{O}_{\widetilde{Y}}(H-\right.$ $\left.3 h), \mathcal{O}_{\widetilde{Y}}(H-2 h)\right)$ and $\left(\pi^{*} \Omega_{\mathbb{P}(W)}, \mathcal{O}_{\widetilde{Y}}(-h)\right)$ on $\tilde{Y}$.

Lemma A.6. There exist a unique extension

$$
0 \rightarrow\left(\mathcal{O}_{\widetilde{Y}}(H-3 h), \mathcal{O}_{\widetilde{Y}}(H-2 h)\right) \rightarrow\left(\tilde{F}_{1}, \tilde{F}_{2}\right) \rightarrow\left(\pi^{*} \Omega_{\mathbb{P}(W)}, \mathcal{O}_{\widetilde{Y}}(-h)\right) \rightarrow 0
$$

in the category of families of Q-representations on $\tilde{Y}$ such that the cone of the induced morphism $\tilde{e}$ : $E_{1} \otimes \tilde{F}_{1} \rightarrow E_{2} \otimes \tilde{F}_{2}$ is a coherent sheaf supported scheme-theoretically at $\tilde{Q}$.

Proof: Since $\left(E_{1}, E_{2}\right)$ is an exceptional pair, $\operatorname{Hom}\left(E_{1}, E_{2}\right)=W^{*}$, and the sheaf of homomorphisms of Q-representations from $\left(\pi^{*} \Omega_{\mathbb{P}(W)}, \mathcal{O}_{\widetilde{Y}}(-h)\right)$ to $\left(\mathcal{O}_{\widetilde{Y}}(H-3 h), \mathcal{O}_{\widetilde{Y}}(H-2 h)\right)$ equals zero, we have a bijection between the set of all extensions (53) in the category of families of Q-representations on $\tilde{Y}$ and $\operatorname{Ext}^{1}\left(G^{\prime \prime}, G^{\prime}\right)=\operatorname{Hom}\left(G^{\prime \prime}[-1], G^{\prime}\right)$. Moreover, it is clear that the cone of $\tilde{e}$ is isomorphic to the cone of the corresponding morphism $\varepsilon: G^{\prime \prime}[-1] \rightarrow G^{\prime}$. Thus we have to construct a morphism $\varepsilon: G^{\prime \prime}[-1] \rightarrow G^{\prime}$, such that the cone of $\varepsilon$ is a sheaf supported scheme-theoretically at $\tilde{Q}$. This can be done as follows.

Let $\tilde{\imath}: \tilde{Q} \rightarrow X \times \tilde{Y}$ denote the embedding. Consider the diagram

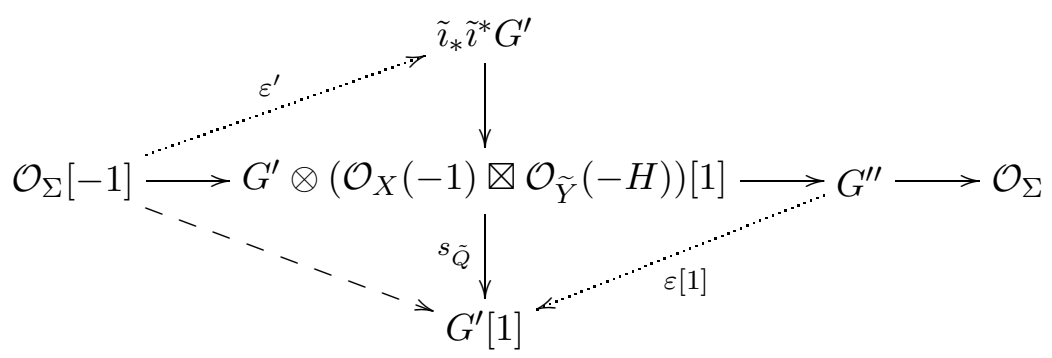

(the horizontal line is the exact triangle (52) and the vertical line is the exact triangle obtained by tensoring the resolution of $\tilde{\imath}_{*} \mathcal{O}_{\tilde{Q}}$ with $G^{\prime}$ ). It follows from lemma A.5 that the dashed arrow is zero. Hence there exist dotted arrows making the diagram commutative. Moreover, since $\operatorname{codim}_{X \times \widetilde{Y}} \Sigma=3$ it follows that the functor $\operatorname{Ext}^{\leq 2}\left(\mathcal{O}_{\Sigma},-\right)$ is zero on any locally free sheaf on $X \times \tilde{Y}$, hence $\operatorname{Hom}\left(\mathcal{O}_{\Sigma}, G^{\prime}[1]\right)=$ $\operatorname{Ext}^{1}\left(\mathcal{O}_{\Sigma}, G^{\prime}\right)=0$, since $G^{\prime}$ admits a locally free resolution of length 2 . Therefore the dotted arrows are unique.

Further, the octahedron axiom implies that the cone of $\varepsilon$ is isomorphic to the cone of $\varepsilon^{\prime}$. Thus it remains to check two things: that $\varepsilon^{\prime}=\tilde{\imath}_{*} \varepsilon^{\prime \prime}$ for some $\varepsilon^{\prime \prime} \in \operatorname{Hom}\left(\mathcal{O}_{\Sigma}[-1], \tilde{\imath}^{*} G^{\prime}\right)$, and that $\tilde{\imath}^{*} G^{\prime}$ is a coherent sheaf. But by definition $\varepsilon^{\prime}$ is obtained from an element of the space

$$
\begin{aligned}
\operatorname{Hom}\left(\tilde{\imath}_{*} \mathcal{O}_{\Sigma}[-1], G^{\prime} \otimes\left(\mathcal{O}_{X}(-1) \otimes \mathcal{O}_{\tilde{Y}}(-H)\right)[1]\right)= & \\
& =\operatorname{Hom}\left(\mathcal{O}_{\Sigma}[-1], \tilde{\imath}^{!}\left(G^{\prime} \otimes\left(\mathcal{O}_{X}(-1) \otimes \mathcal{O}_{\tilde{Y}}(-H)\right)[1]\right)\right) \cong \operatorname{Hom}\left(\mathcal{O}_{\Sigma}[-1], \tilde{\imath}^{*} G^{\prime}\right),
\end{aligned}
$$

so the first is true. For the second we must check that $\operatorname{Ker} \tilde{\imath}^{*} \tilde{e}^{\prime}=0$. But the second exact sequence of lemma A.3 shows that $\operatorname{Ker} \tilde{\imath}^{*} \tilde{e}^{\prime} \cong \mathcal{H}^{-3}\left(\tilde{\imath}^{*}\left(\mathcal{O}_{\Sigma} \otimes\left(\mathcal{O}_{X}(1) \otimes \mathcal{O}_{\widetilde{Y}}(H)\right)\right)\right)$ which is zero because the Tor-dimension of $\tilde{\imath}$ equals 1 .

Lemma A.6 says that the family $\left(\tilde{F}_{1}, \tilde{F}_{2}\right)$ on $\tilde{Y}$ is a family of Q-representations and that on $X \times \tilde{Y}$ we have the following exact sequence:

$$
0 \rightarrow E_{1} \otimes \tilde{F}_{1} \stackrel{\tilde{e}}{\longrightarrow} E_{2} \otimes \tilde{F}_{2} \rightarrow \tilde{\imath}_{*} \tilde{\mathcal{E}} \rightarrow 0,
$$

where $\tilde{\mathcal{E}}$ is a coherent sheaf on $\tilde{Q}$.

Lemma A.7. The family $\left(\tilde{F}_{1}, \tilde{F}_{2}\right)$ induces a morphism $\phi: \tilde{Y} \rightarrow \bar{Y}_{2}$ such that $\tilde{g}=\bar{g}_{2} \circ \phi$. 
Proof: For any point $y \in \widetilde{Y}$ we choose a trivializations of $F_{1}$ and $F_{2}$ in a small neighborhood of $y$. This induces a morphism $\tilde{\phi}$ of this neighborhood into a representation space of the quiver Q. Since $\operatorname{rank}\left(\tilde{F}_{1}\right)=6, \operatorname{rank}\left(\tilde{F}_{2}\right)=2$, and $\operatorname{gcd}(6,2)=2$ this is the space $\mathbf{R}_{2}$. Let $y^{\prime}$ be an arbitrary point of the neighborhood. Let us check that $\tilde{\phi}\left(y^{\prime}\right) \in \mathbf{R}_{2}^{\circ}$. Indeed, this is equivalent to the injectivity of the map $\eta^{*} \tilde{e}$, where $\eta: X \times y^{\prime} \rightarrow X \times \tilde{Y}$ is the embedding, or equivalently to $\eta^{*} \tilde{\imath}_{*} \tilde{\mathcal{E}} \in \mathcal{D}^{\geq 0}$. But it was shown in the proof of lemma A.6 that we have an exact triangle

$$
\mathcal{O}_{\Sigma}[-1] \stackrel{\varepsilon^{\prime}}{\longrightarrow} \tilde{i}_{*} \tilde{\imath}^{*} G^{\prime} \longrightarrow \tilde{\imath}_{*} \tilde{\mathcal{E}}
$$

Thus it suffices to check that $\eta^{*} \tilde{\imath}_{*} \tilde{\imath}^{*} G^{\prime} \in \mathcal{D}^{\geq 0}$ and $\eta^{*} \mathcal{O}_{\Sigma} \in \mathcal{D}^{\geq 0}$. Note that the following diagrams are exact cartesian by 2.27
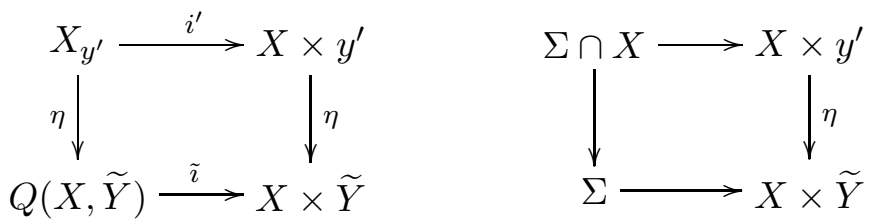

and the top arrows are closed embeddings. The second inclusion follows immediately and for the first we note that by (52) we have $\mathcal{H}^{-1}\left(\eta^{*} \tilde{\imath}_{*} \tilde{\imath}^{*} G^{\prime} \otimes \mathcal{O}_{X}(-1) \otimes \mathcal{O}_{\tilde{Y}}(-H)\right)=\mathcal{H}^{-3}\left(\eta^{*} \tilde{\imath}_{*} \tilde{\imath}^{*} \mathcal{O}_{\Sigma}\right)$ and on the other hand $\eta^{*} \tilde{i}_{*} \tilde{\imath}^{*} \mathcal{O}_{\Sigma}=i_{*}^{\prime} \eta^{*} \tilde{\imath}^{*} \mathcal{O}_{\Sigma}=i_{*}^{\prime} i^{\prime *} \eta^{*} \mathcal{O}_{\Sigma}=i_{*}^{\prime} i^{\prime *} \mathcal{O}_{\Sigma \cap X} \in \mathcal{D}^{\geq-1}$

Further, supp $\eta^{*} \tilde{\imath}_{*} \tilde{\mathcal{E}}=\operatorname{supp} i_{*}^{\prime} \eta^{*} \tilde{\mathcal{E}}=X_{y^{\prime}}$, is a hyperplane section of $X$, hence $\tilde{\phi}\left(y^{\prime}\right) \in \mathbf{R}_{2}^{1}$.

Composing $\tilde{\phi}$ with the factorization map $\mathbf{R}_{2}^{1} \rightarrow \bar{Y}_{2}=\mathbf{R}_{2}^{1} / / \chi G$ we obtain a map $\phi_{y}$ from the neighborhood of $y$ in $\widetilde{Y}$ to $\bar{Y}_{2}$. It is clear that $\phi_{y}$ doesn't depend on a choice of trivializations, hence constructed morphisms $\phi_{y}$ glue into a morphism $\phi: \tilde{Y} \rightarrow \bar{Y}_{2}$. Finally, since $\tilde{\imath}_{*} \tilde{\mathcal{E}}$ is supported on $\tilde{Q}$ it follows that $\tilde{g}=\bar{g}_{2} \circ \phi$.

Let $\tilde{Y}^{\circ}=\widetilde{Y} \backslash \tilde{g}^{-1}\left(Z_{1}\right)$ and denote the restriction of $\tilde{g}$ and $\phi$ to $\tilde{Y}^{\circ}$ by the same symbols.

Lemma A.8. The map $\tilde{g}: \tilde{Y}^{\circ} \rightarrow \mathbb{P}\left(V^{*}\right) \backslash Z_{1}$ factors through $X^{\vee} \backslash Z_{1}$, where $X^{\vee} \subset \mathbb{P}\left(V^{*}\right)$ is the projectively dual quartic hypersurface. Moreover, the general fiber of $\tilde{g}: \tilde{Y}^{\circ} \rightarrow X^{\vee} \backslash Z_{1}$ is a conic.

Proof: Let $H_{\tilde{g}\left(y^{\prime}\right)} \subset \mathbb{P}(V)$ be the hyperplane corresponding to a point $\tilde{g}\left(y^{\prime}\right) \in \mathbb{P}\left(V^{*}\right)$. It is clear from the proof of lemma A.7 that for any point $y^{\prime} \in \widetilde{Y}$ we have

$$
X \cap H_{\tilde{g}\left(y^{\prime}\right)}=X_{y^{\prime}}=\eta^{-1}(\tilde{Q}) \supset \eta^{-1}(\Sigma)=\operatorname{LGr}(2,4),
$$

But a smooth hyperplane section of $X=\operatorname{LGr}(3,6)$ cannot contain a $\operatorname{LGr}(2,4)$ by Lefschetz theorem. Hence $X \cap H_{\tilde{g}\left(y^{\prime}\right)}$ is singular for any $y^{\prime} \in \tilde{Y}$, hence $\tilde{g}(\widetilde{Y}) \subset X^{\vee} \subset \mathbb{P}\left(V^{*}\right)$. Moreover, it is easy to compute the intersection index $H^{12} \cdot h=8$ on $\tilde{Y}$, which implies that the map $\tilde{g}: \tilde{Y} \rightarrow X^{\vee}$ is surjective and its generic fiber is a conic in $\mathbb{P}(W)$, because $X^{\vee}$ is a quartic hypersurface by [Ho].

Recall that we have a sheaf of Azumaya algebras $\mathcal{A}_{2}$ on $Y_{2}$ and that $F_{2}$ is a locally projective $\mathcal{A}_{2}$-module on $Y_{2}$ of rank 2. Let $\mathbb{P}_{Y_{2}, \mathcal{A}_{2}}\left(F_{2}^{*}\right)$ denote the moduli space of locally projective quotient $\mathcal{A}_{2}$-modules of rank 1. Then $\mathbb{P}_{Y_{2}, \mathcal{A}_{2}}\left(F_{2}^{*}\right)$ is a conic bundle over $Y_{2}$.

Proposition A.9. We have $\widetilde{Y}^{\circ}=\mathbb{P}_{Y_{2}, \mathcal{A}_{2}}\left(F_{2}^{*}\right)$.

Proof: Let $y \in \tilde{Y}^{\circ}$. Then $\phi(y) \in \bar{Y}_{2}$ and $\bar{g}_{2}(\phi(y))=\tilde{g}(y) \notin Z_{1}$, hence $\phi(y) \in Y_{2}$. Therefore $\phi$ takes $\tilde{Y}^{\circ}$ to $Y_{2}$. Moreover, it follows from the construction of the map $\phi$ in lemma A.7 and from the construction of the family $\left(F_{1}, F_{2}\right)$ on $Y_{2}$ in lemma 5.6 that $\phi^{*} F_{2} \cong \tilde{F}_{0}^{*} \otimes \tilde{F}_{2}$ and $\phi^{*} \mathcal{A}_{2} \cong \mathcal{E} n d\left(\tilde{F}_{0}\right)$ for a certain vector bundle $\tilde{F}_{0}$ on $\tilde{Y}^{\circ}$. The epimorphism $\tilde{F}_{2} \rightarrow \mathcal{O}_{\tilde{Y}}(-h)$ from $(53)$ gives an epimorphism of $\mathcal{E} n d\left(\tilde{F}_{0}\right)$-modules $\tilde{F}_{0}^{*} \otimes \tilde{F}_{2} \rightarrow \tilde{F}_{0}^{*}(-h)$, hence induces a morphism $\tilde{Y}^{\circ} \rightarrow \mathbb{P}_{Y_{2}, \mathcal{A}_{2}}\left(F_{2}^{*}\right)$. 
Vice versa, choose a point $\tilde{y} \in \mathbb{P}_{Y_{2}, \mathcal{A}_{2}}\left(F_{2}^{*}\right)$. Let $y \in Y_{2}$ be its projection and let $\rho: W \otimes R_{1} \rightarrow R_{2}$ be the corresponding (6,2)-dimensional representation of the quiver $\mathrm{Q}$, and $R_{2} \rightarrow \mathrm{k}$ - the corresponding onedimensional quotient. Let $R_{2}^{\prime} \subset R_{2}$ be its kernel and consider the composition $E_{1} \otimes R_{1} \stackrel{e_{\rho}}{\longrightarrow} E_{2} \otimes R_{2} \rightarrow E_{2}$. Applying $\operatorname{Hom}\left(E_{1},-\right)$ we obtain a map $R_{1} \rightarrow W^{*}$. Let $R_{1}^{\prime}$ denote the kernel of this map. Then we have a diagram

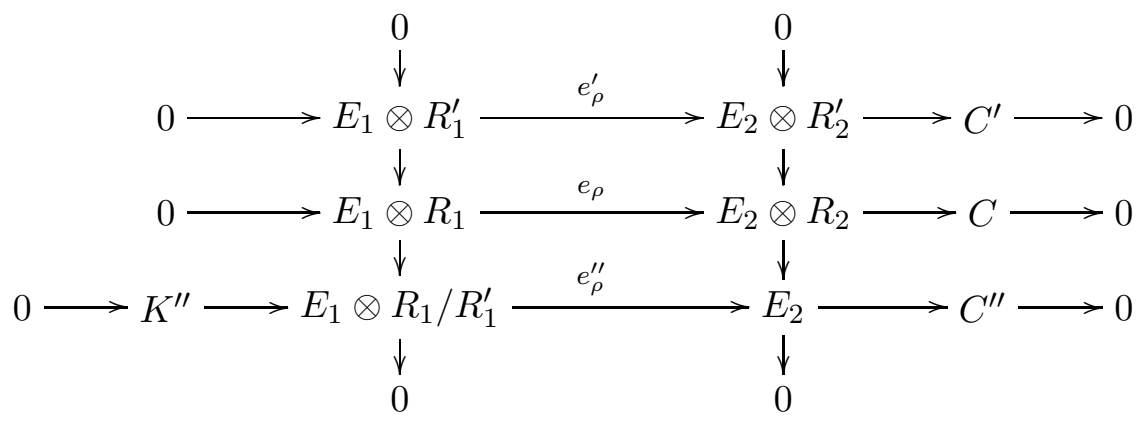

which induces an exact sequence

$$
0 \rightarrow K^{\prime \prime} \rightarrow C^{\prime} \rightarrow C \rightarrow C^{\prime \prime} \rightarrow 0
$$

Note that the sheaf $C$ is supported on the hyperplane section $X_{y}=H_{g_{2}(y)} \cap X \subset X$ and its rank at the generic point of $X_{y}$ equals $c_{1}\left(E_{2} \otimes R_{2}\right)-c_{1}\left(E_{1} \otimes R_{1}\right)=2$.

We claim that $\operatorname{dim} R^{\prime}=1$ and we are going to prove it by a case by case analysis.

Since $\left(R_{1}^{\prime}, R_{2}^{\prime}\right)$ is a subrepresentation of $\left(R_{1}, R_{2}\right)$ and $\left(R_{1}, R_{2}\right)$ is stable by lemma 5.5 we have

$$
\operatorname{dim} R_{1}^{\prime}<3
$$

so we need to reject two cases: $\operatorname{dim} R_{1}^{\prime}=2$ and $\operatorname{dim} R_{1}^{\prime}=0$.

Assume that $\operatorname{dim} R_{1}^{\prime}=2, \operatorname{dim} R_{1} / R_{1}^{\prime}=4$. Then the bottom line of the diagram (54) takes form

$$
0 \rightarrow K^{\prime \prime} \rightarrow\left(R_{1} / R_{1}^{\prime}\right) \otimes \mathcal{O}_{X} \stackrel{e^{\prime \prime}}{\rightarrow} \mathcal{U}_{X}^{*} \rightarrow C^{\prime \prime} \rightarrow 0 .
$$

Since $C^{\prime \prime}$ is a quotient of $C$ by $(55)$ we have $\operatorname{rank}\left(C^{\prime \prime}\right)=0$, hence $\operatorname{rank}\left(K^{\prime \prime}\right)=4-3=1$. Thus $K^{\prime \prime}$ is a reflexive sheaf of rank 1 , hence $K^{\prime \prime}$ is a line bundle by [OSS]. Since Pic $X$ is generated by $\mathcal{O}_{X}(1)$ we have $K^{\prime \prime} \cong \mathcal{O}_{X}(-k), k \geq 0$. Moreover, $k \neq 0$ since the map $H^{0}\left(e^{\prime \prime}\right)$ is an embedding (by definition of $\left.R_{1}^{\prime}\right)$. On the other hand, $-k=c_{1}\left(K^{\prime \prime}\right)=c_{1}\left(C^{\prime \prime}\right)-c_{1}\left(\mathcal{U}_{X}^{*}\right) \geq-c_{1}\left(\mathcal{U}_{X}\right)^{*}=-1$, hence $k=1$ and $c_{1}\left(C^{\prime \prime}\right)=c_{1}\left(K^{\prime \prime}\right)+c_{1}\left(\mathcal{U}_{X}^{*}\right)=0$. Therefore, the rank of $C^{\prime \prime}$ at the generic point of $X_{y}$ is zero.

Further, the top line of the diagram (54) takes form

$$
0 \rightarrow R_{1}^{\prime} \otimes \mathcal{O}_{X} \stackrel{e^{\prime}}{\rightarrow} \mathcal{U}_{X}^{*} \rightarrow C^{\prime} \rightarrow 0 .
$$

We have $\operatorname{rank}\left(C^{\prime}\right)=\operatorname{rank}\left(\mathcal{U}_{X}^{*}\right)-\operatorname{dim} R_{1}^{\prime}=1$, and $c_{1}\left(C^{\prime}\right)=c_{1}\left(\mathcal{U}_{X}^{*}\right)=1$. If $T\left(C^{\prime}\right)$ denotes the torsion part of $C^{\prime}$ then $C^{\prime} / T\left(C^{\prime}\right)$ is a torsion free rank 1 sheaf, hence $C^{\prime} / T\left(C^{\prime}\right) \cong J_{S}(k)$ (see [OSS]), where $S \subset X$ is a subscheme of codimension $\geq 2$ in $X$. Therefore $c_{1}\left(C^{\prime}\right)=c_{1}\left(T\left(C^{\prime}\right)\right)+k \geq k$, so $k \leq 1$. On the other hand, $k \geq 1$ since we have a nontrivial morphism $\mathcal{U}_{X}^{*} \rightarrow C^{\prime} \rightarrow J_{S}(k) \rightarrow \mathcal{O}_{X}(k)$. Therefore, $k=1$, $c_{1}\left(T\left(C^{\prime}\right)\right)=c_{1}\left(C^{\prime}\right)-k=0$, hence the torsion part of $C^{\prime}$ is supported in codimension $\geq 2$ and the rank of $C^{\prime}$ at the generic point of $X_{y}$ equals $\operatorname{rank}\left(C^{\prime}\right)=1$.

Now we have shown that the rank of $C^{\prime \prime}$ at the generic point of $X_{y}$ equals 0 and the rank of $C^{\prime}$ at the generic point of $X_{y}$ equals 1 . Looking at (55) we see that this contradicts to the fact that the rank of $C$ at the generic point of $X_{y}$ equals 2 . So the case $\operatorname{dim} R_{1}^{\prime}=2$ is impossible.

Assume that $R_{1}^{\prime}=0$. Then the map $R_{1} \rightarrow W^{*}$ is injective, hence an isomorphism since $\operatorname{dim} R_{1}=6=$ $\operatorname{dim} W^{*}$. Thus $R_{1} / R_{1}^{\prime} \cong W^{*}$ and the map $e_{\rho}^{\prime \prime}$ gets identified with the canonical morphism $W^{*} \otimes \mathcal{O}_{X} \rightarrow \mathcal{U}_{X}^{*}$. 
Therefore, $C^{\prime \prime}=0$ and $K^{\prime \prime}=\mathcal{U}_{X}^{\perp} \cong \mathcal{U}_{X}$. On the other hand, it is clear that $C^{\prime}=R_{2}^{\prime} \otimes \mathcal{U}_{X}^{*} \cong \mathcal{U}_{X}^{*}$. Thus the sequence (55) takes form

$$
0 \rightarrow \mathcal{U}_{X} \rightarrow \mathcal{U}_{X}^{*} \rightarrow C \rightarrow 0 .
$$

Note that $\operatorname{Hom}\left(\mathcal{U}_{X}, \mathcal{U}_{X}^{*}\right) \cong S^{2} W^{*} \oplus \Lambda^{2} W^{*} /$ k. If a homomorphism has a nontrivial component in $S^{2} W^{*}$ then its cokernel is supported on a hyperquadric section of $X$, and if a homomorphism lies in $\Lambda^{2} W^{*} / \mathrm{k}$ then it is skew-symmetric, hence degenerate (since $\mathcal{U}_{X}$ has odd rank). Thus $C$ cannot be a rank 2 sheaf on a hyperplane section of $X$. So the case $\operatorname{dim} R_{1}^{\prime}=2$ is impossible as well.

Therefore, $\operatorname{dim} R_{1}^{\prime}=1, \operatorname{dim} R_{1} / R_{1}^{\prime}=5$. The top and the bottom lines of the diagram (54) take form

$$
\begin{gathered}
0 \rightarrow \mathcal{O}_{X} \stackrel{e^{\prime}}{\longrightarrow} \mathcal{U}_{X}^{*} \rightarrow C^{\prime} \rightarrow 0, \\
0 \rightarrow K^{\prime \prime} \rightarrow \mathcal{O}_{X}^{\oplus 5} \stackrel{e^{\prime \prime}}{\longrightarrow} \mathcal{U}_{X}^{*} \rightarrow C^{\prime \prime} \rightarrow 0 .
\end{gathered}
$$

The map $e^{\prime}$ is given by an element $w^{\prime} \in \mathbb{P}\left(\operatorname{Hom}\left(\mathcal{O}_{X}, \mathcal{U}_{X}^{*}\right)\right)=\mathbb{P}\left(W^{*}\right)$ and the map $e^{\prime \prime}$ is given by a hyperplane in $\mathbb{P}\left(W^{*}\right)$, that is by a point $w^{\prime \prime} \in \mathbb{P}(W)$. Moreover, it follows from the canonical exact sequence $0 \rightarrow \mathcal{U}_{X} \rightarrow W^{*} \otimes \mathcal{O}_{X} \rightarrow \mathcal{U}_{X}^{*} \rightarrow 0$ that we have the following exact sequence

$$
0 \rightarrow K^{\prime \prime} \rightarrow \mathcal{U}_{X} \stackrel{w^{\prime \prime}}{\longrightarrow} \mathcal{O}_{X} \rightarrow C^{\prime \prime} \rightarrow 0 .
$$

In particular, $K^{\prime \prime}$ is reflexive and supp $C^{\prime \prime}=\operatorname{LGr}(2,4)$ and has codimension 3. Moreover, it follows from (56) that $C^{\prime}$ is reflexive and $\operatorname{rank}\left(C^{\prime}\right)=2$. Therefore $C_{\mid X_{y}}^{\prime}$ is torsion free of rank 2 on $X_{y}$ and we have the following exact sequence

$$
0 \rightarrow C^{\prime}(-1) \rightarrow C^{\prime} \rightarrow C_{\mid X_{y}}^{\prime} \rightarrow 0
$$

Now, note that since $C$ is supported on $X_{y}$ the map $C^{\prime} \rightarrow C$ factors through $C_{\mid X_{y}}^{\prime}$. Since the ranks of both $C_{\mid X_{y}}^{\prime}$ and $C$ at generic points of $X_{y}$ equal to 2 and the rank of $C^{\prime \prime}$ at generic point of $X_{y}$ is zero, it follows that the map $C_{\mid X_{y}}^{\prime} \rightarrow C$ is an embedding. Therefore, the composition $K^{\prime \prime} \rightarrow C^{\prime} \rightarrow C_{\mid X_{y}}^{\prime}$ is zero, hence the map $K^{\prime \prime} \rightarrow C^{\prime}$ factors through the map $K^{\prime \prime} \rightarrow C^{\prime}(-1)$. But this map, being an embedding of reflexive sheaves of equal rank and $c_{1}$ is an isomorphism. An isomorphism $K^{\prime \prime} \cong C^{\prime}(-1)$ allows to glue the sequence (56) twisted by $\mathcal{O}_{X}(-1)$ with the sequence (57) into the following exact sequence

$$
0 \rightarrow \mathcal{O}_{X}(-1) \stackrel{w^{\prime}}{\longrightarrow} \mathcal{U}_{X}^{*}(-1) \rightarrow \mathcal{U}_{X} \stackrel{w^{\prime \prime}}{\longrightarrow} \mathcal{O}_{X} \rightarrow C^{\prime \prime} \rightarrow 0,
$$

Then it is easy to see that this sequence is the Koszul complex of $w^{\prime \prime}$, hence $w^{\prime}=w^{\prime \prime}$ and $C^{\prime \prime} \cong \mathcal{O}_{Z\left(w^{\prime \prime}\right)}$, where $Z\left(w^{\prime \prime}\right)$ is the zero locus of $w^{\prime \prime} \in H^{0}\left(X, \mathcal{U}_{X}^{*}\right)$. It is easy to check that the space of hyperplane section of $X$ passing through $Z\left(w^{\prime \prime}\right)$ equals to the fiber of the bundle $\Omega_{\mathbb{P}(W)}^{3}(3) / \mathcal{O}_{\mathbb{P}(W)}(-1) \subset V^{*} \otimes \mathcal{O}_{\mathbb{P}(W)}$ at $w^{\prime \prime}$. On the other hand, the support hyperplane $X_{y}$ of $C$ contains supp $\left(C^{\prime \prime}\right)=Z\left(w^{\prime \prime}\right)$, therefore the map

$$
\mathbb{P}_{Y_{2}, \mathcal{A}_{2}}\left(F_{2}^{*}\right) \rightarrow \mathbb{P}(W) \times \mathbb{P}\left(V^{*}\right), \quad \tilde{y} \mapsto\left(w^{\prime \prime}, g_{2}(y)\right)
$$

factors through $\tilde{Y}=\mathbb{P}_{\mathbb{P}(W)}\left(\Omega_{\mathbb{P}(W)}^{3}(3) / \mathcal{O}_{\mathbb{P}(W)}(-1)\right) \subset \mathbb{P}(W) \times \mathbb{P}\left(V^{*}\right)$. Moreover, it is clear that its image lies in $\tilde{Y}^{\circ}$, so we have constructed a map $\mathbb{P}_{Y_{2}, \mathcal{A}_{2}}\left(F_{2}^{*}\right) \rightarrow \widetilde{Y}^{\circ}$.

It remains to note that the constructed maps are mutually inverse.

Theorem A.10. We have $Y_{2}=X^{\vee} \backslash Z_{1}$.

Proof: Since $\tilde{Y}^{\circ}$ is smooth it follows from proposition A.9 that $Y_{2}$ is smooth and moreover $\operatorname{dim} Y_{2}=$ $\operatorname{dim} \tilde{Y}^{\circ}-1=12$. On the other hand, since $\tilde{g}=\bar{g}_{2} \circ \phi, \tilde{g}$ and $\phi$ are proper and dominant, and $\tilde{g}$ takes $\tilde{Y}^{\circ}$ to $X^{\vee} \backslash Z_{1}$, it follows that $g_{2}$ is a proper dominant map $Y_{2} \rightarrow X^{\vee} \backslash Z_{1}$. On the other hand, by [Ho] we have $Z_{1}=\operatorname{sing}\left(X^{\vee}\right)$, hence $X^{\vee} \backslash Z_{1}$ is also smooth and $\operatorname{dim}\left(X^{\vee} \backslash Z_{1}\right)=\operatorname{dim} \mathbb{P}\left(V^{*}\right)-1=12$. So, $g_{2}: Y_{2} \rightarrow X^{\vee} \backslash Z_{1}$ is a proper dominant map of smooth varieties of equal dimension. Therefore, either $Y_{2}=X^{\vee} \backslash Z_{1}$, or rankPic $Y_{2}=\operatorname{rank} \operatorname{Pic} X^{\vee}+1=2$. Assume that the latter is true, and note that since 
$\phi$ is a conic bundle, we have rank $\operatorname{Pic} \widetilde{Y}^{\circ}=\operatorname{rank} \operatorname{Pic} Y_{2}+1=3$. But on the other hand, it is easy to see that rankPic $\tilde{Y}^{\circ} \leq \operatorname{rank} \operatorname{Pic} \tilde{Y}=2$. Thus we get a contradiction, hence $Y_{2}=X^{\vee} \backslash Z_{1}$.

Corollary A.11. If the data (D.3)-(D.5) is given by $Y=Y_{2}, \mathcal{A}_{Y}=\mathcal{A}_{2}, \mathbf{Z}=\bar{Z}_{2}=Z_{1}, g=g_{2}$, with $\left(F_{1}, F_{2}\right)$ being the universal family, and with $\phi$ being induced by the Q-representation structure on $\left(F_{1}, F_{2}\right)$, then all conditions (C.1)-(C.8) are satisfied.

Proof: Since conditions (45) and (51) are satisfied by lemma A.1, it follows from theorem 5.7 that conditions (C.4)-(C.6) are true. On the other hand, (C.8) is true because $\mathbf{Z}=\mu\left(\mathrm{Gr}\left(3, W^{*}\right)\right)$ by lemma A.2 and $\operatorname{dim} \mathbf{Z} \cap H=\operatorname{dim} \operatorname{Gr}\left(3, W^{*}\right)-1=8$, while $N-i-2=14-4-2=8$. Finally, by theorem A.10 the map $Y=Y_{2} \rightarrow \mathbb{P}\left(V^{*}\right)$ is an embedding and $\operatorname{codim}_{Y \times Y}\left(Y \times_{\mathbb{P}\left(V^{*}\right)} Y\right)=\operatorname{dim} Y=\operatorname{dim} X^{\vee}=\operatorname{dim} \mathbb{P}\left(V^{*}\right)-1=12$, hence $\operatorname{dim} X+N-\operatorname{codim}_{Y \times Y}\left(Y \times_{\mathbb{P}\left(V^{*}\right)} Y\right)=6+14-12=8=2 i$, and (C.7) is true.

\section{Appendix B. $G_{2}$ Grassmannian}

In this appendix we show that a double covering of $\mathbb{P}^{13}$ ramified in a sextic hypersurface is homologically projectively dual to the Grassmannian $\mathrm{G}_{2} \mathrm{Gr}(2,7)$ of the simple Lie group $G_{2}$.

Recall the notation. Let $W=\mathrm{k}^{7}$ be an irreducible representation of the simple Lie group $G_{2}$ and

$$
X=\mathrm{G}_{2} \operatorname{Gr}(2, W), \quad\left(E_{1}, E_{2}\right)=\left(\mathcal{O}_{X}, \mathcal{U}_{X}^{*}\right),
$$

where $G_{2} \operatorname{Gr}(2, W)$ is the Grassmannian of the Lie group $G_{2}$, realized as the zero locus of the section $s_{\lambda} \in H^{0}\left(\operatorname{Gr}(2, W), \mathcal{U}^{\perp}(1)\right) \cong \Lambda^{3} W^{*}$, corresponding to the unique $G_{2}$-invariant 3 -form $\lambda$ on $W, \mathcal{U} \subset$ $W \otimes \mathcal{O}_{\mathrm{Gr}(2, W)}$ is the tautological rank 2 subbundle, and $\mathcal{U}_{X}$ is the restriction of $\mathcal{U}$ to $X$. The natural representation of the group $G_{2}$ in the space $\Lambda^{2} W$ decomposes into the direct sum of representations

$$
\Lambda^{2} W=W^{*} \oplus V
$$

(the projection $\Lambda^{2} W \rightarrow W^{*}$ is given by the 3 -form $\lambda$ ). The Plücker embedding $\operatorname{Gr}(2, W) \subset \mathbb{P}\left(\Lambda^{2} W\right)$ restricts to an embedding $f: X \rightarrow \mathbb{P}(V)$.

Lemma B.1. We have $\operatorname{dim} X=5, \omega_{X} \cong \mathcal{O}_{X}(-3)$. Moreover, conditions (C.1)-(C.3) as well as the additional conditions (45) and (51) are satisfied for $X$.

Proof: Note that $X \subset \operatorname{Gr}(2, W)$ is the zero locus of a regular section $s_{\lambda} \in H^{0}\left(\operatorname{Gr}(2, W), \mathcal{U}^{\perp}(1)\right)$. Hence

$$
\begin{aligned}
& \operatorname{dim} X=\operatorname{dim} \operatorname{Gr}(2, W)-\operatorname{rank}\left(\mathcal{U}^{\perp}(1)\right)=10-5=5, \\
& \omega_{X} \cong \omega_{\operatorname{Gr}(2, W) \mid X} \otimes \operatorname{det}\left(\mathcal{U}^{\perp}(1)\right)^{*} \cong \mathcal{O}_{X}(-7) \otimes \mathcal{O}_{X}(4) \cong \mathcal{O}_{X}(-3) .
\end{aligned}
$$

In particular, (C.2) is true and $i_{X}=3$. Further, using the Koszul resolution $\Lambda^{\bullet}\left(\mathcal{U}^{\perp}(1)\right) \cong \mathcal{O}_{X}$ of $X$ in $\operatorname{Gr}(2, W)$ it is easy to check that $H^{0}\left(X, \mathcal{O}_{X}(1)\right) \cong V^{*}$, hence $f(X)$ is not contained in a hyperplane, i.e. (C.1) is true. Using again the Koszul resolution we compute $\operatorname{Hom}\left(E_{s}(k), E_{t}(l)\right)$ via Borel-Bott-Weil theorem and check that (C.3) and (45) are true. Finally, we note that $\mathbf{R}_{1}=\operatorname{Hom}\left(\mathrm{k}^{2}, W^{*}\right)$ and $\mathbf{R}_{1}^{\circ} \subset \mathbf{R}_{1}$ corresponds to the subset of embeddings $\mathrm{k}^{2} \subset W^{*}$. Therefore, $\operatorname{codim}_{\mathbf{R}_{1}}\left(\mathbf{R}_{1} \backslash \mathbf{R}_{1}^{\circ}\right)=6$ and (51) is satisfied by lemma 5.3 .

Now we are going to apply the construction of section 5 and to describe the space $Y=Y_{3}$ explicitly. We use the same approach as in Appendix A. The arguments in most cases are the same. So we will not repeat them, but describe the differences.

Instead of $Y_{3}$ we will describe $\mathbb{P}_{Y_{3}, \mathcal{A}_{3}}\left(F_{2}^{*}\right)$, the moduli space of quotient $\mathcal{A}_{3}$-modules of rank 1 . The fiber of $\mathbb{P}_{Y_{3}, \mathcal{A}_{3}}\left(F_{2}^{*}\right)$ over a point of $Y_{3}$ corresponding to a $(6,3)$-dimensional representation $\rho=\left(R_{1}, R_{2}\right)$ of $\mathrm{Q}$ coincides with $\mathbb{P}\left(R_{2}^{*}\right)$. We show that any plane $R_{2}^{\prime} \subset R_{2}$ can be extended in a unique way to a $(1,2)$ dimensional subrepresentation $\rho^{\prime}=\left(R_{1}^{\prime}, R_{2}^{\prime}\right)$. The moduli space of $(1,2)$-dimensional representations of 
$\mathrm{Q}$ is $\operatorname{Gr}\left(2, W^{*}\right)$. We claim that defined in this way map $\mathbb{P}_{Y_{3}, \mathcal{A}_{3}}\left(F_{2}^{*}\right) \rightarrow \operatorname{Gr}\left(2, W^{*}\right)$ identifies $\mathbb{P}_{Y_{3}, \mathcal{A}_{3}}\left(F_{2}^{*}\right)$ with an open subset in a $\mathbb{P}^{8}$-bundle over $\operatorname{OGr}\left(2, W^{*}\right) \subset \operatorname{Gr}\left(2, W^{*}\right)$, the isotropic Grassmannian.

Lemma B.2. We have $\bar{Y}_{1}=\bar{Z}_{1}=\mu\left(\operatorname{Gr}\left(2, W^{*}\right)\right)$, where $\mu: \mathbb{P}\left(\Lambda^{2} W^{*}\right)--\rightarrow \mathbb{P}\left(V^{*}\right)$ is the linear projection from $\mathbb{P}(\lambda(W)) \subset \mathbb{P}\left(\Lambda^{2} W^{*}\right)$.

Recall that the group $G_{2}$ is a subgroup of the group $\mathrm{O}(W)$. Consider the corresponding $G_{2}$-invariant nondegenerate quadratic form on $W$ and let $\mathcal{Q} \subset \mathbb{P}(W), \operatorname{OGr}(2, W) \subset \operatorname{Gr}(2, W)$ and $\operatorname{OF}(1,2 ; W) \subset$ $\mathrm{FI}(1,2 ; W)$ denote the corresponding quadric, the isotropic Grassmannian and the isotropic partial flag variety. Let $\tilde{p}: \operatorname{OF}(1,2 ; W) \rightarrow \operatorname{OGr}(2, W)$ and $\tilde{q}: \operatorname{OF}(1,2 ; W) \rightarrow \mathcal{Q}$ denote the projections. Let $S_{\mathcal{Q}}$ denote the spinor bundle on $\mathcal{Q}$, let $\mathcal{U}_{\mathrm{OGr}}$ denote the tautological rank 2 subbundle in $W \otimes \mathcal{O}_{\mathrm{OGr}(2, W)}$ and put $M:=\tilde{p}_{*} \tilde{q}^{*} S_{\mathcal{Q}} \in \mathcal{D}(\operatorname{OGr}(2, W))$.

Lemma B.3. We have the following commutative diagram

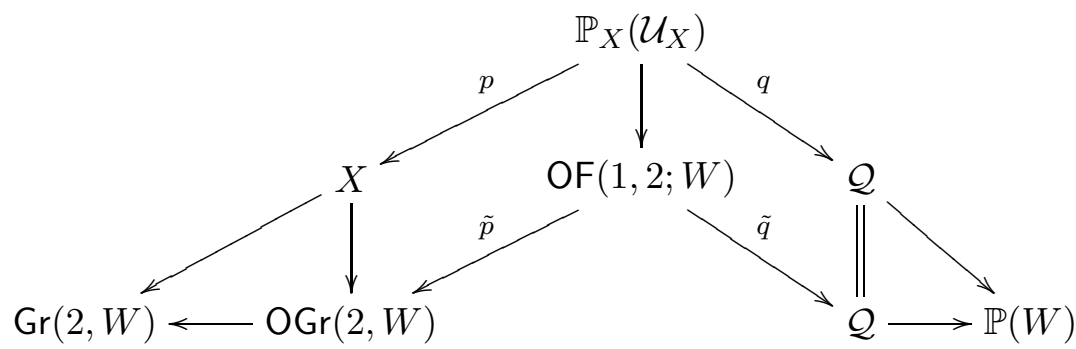

Moreover, $M$ is a rank 2 vector bundle on $\operatorname{OGr}(2, W)$ and we have an isomorphism $M_{\mid X} \cong \mathcal{U}_{X}$ and an exact sequence

$$
0 \rightarrow \mathcal{O}_{\mathrm{OGr}(2, W)}(-1) \rightarrow M \rightarrow \mathcal{O}_{\mathrm{OGr}(2, W)} \rightarrow \mathcal{O}_{X} \rightarrow 0 .
$$

Proof: Note that $\mathbb{P}_{X}\left(\mathcal{U}_{X}\right)$ is the flag variety of the group $G_{2}$. The embedding $G_{2} \subset \mathrm{O}(W)$ induces an embedding of the flag varieties $\mathbb{P}_{X}\left(\mathcal{U}_{X}\right) \rightarrow \operatorname{OF}(1,2 ; W)$, and of the Grassmannians $X \rightarrow \operatorname{OGr}(2, W)$ such that the diagram is commutative (note that the quadric $\mathcal{Q}$ is also a Grassmannian of the group $G_{2}$ ).

The second claim follows from the fact that the restriction of the spinor bundle $S_{\mathcal{Q}}$ to any line $\mathbb{P}^{1} \subset \mathcal{Q}$ splits as $\mathcal{O}_{\mathbb{P}^{1}} \oplus \mathcal{O}_{\mathbb{P}^{1}} \oplus \mathcal{O}_{\mathbb{P}^{1}}(-1) \oplus \mathcal{O}_{\mathbb{P}^{1}}(-1)$.

To check the third claim we note that $\mathbb{P}_{X}\left(\mathcal{U}_{X}\right) \cong \mathbb{P}_{\mathcal{Q}}(K)$, where $K$ is the kernel of the restriction to $\mathcal{Q}$ of the morphism $T_{\mathbb{P}(W)}(-2) \rightarrow \Omega_{\mathbb{P}(W)}(1)$ given by the form $\lambda$. On the other hand, the image of this morphism is just the spinor bundle, so we have an exact sequence

$$
0 \rightarrow K \rightarrow T_{\mathbb{P}(W)}(-2)_{\mid \mathcal{Q}} \rightarrow S_{\mathcal{Q}} \rightarrow 0 .
$$

Taking into account the sequences

$$
\begin{aligned}
& 0 \rightarrow p^{*} \mathcal{O}_{X}(-1) \rightarrow q^{*} K \rightarrow p^{*} \mathcal{O}_{X}(1) \otimes q^{*} \mathcal{O}_{\mathcal{Q}}(-3) \rightarrow 0 \\
& 0 \rightarrow q^{*} \mathcal{O}_{\mathcal{Q}}(-1) \rightarrow p^{*} \mathcal{U}_{X} \rightarrow q^{*} \mathcal{O}_{\mathcal{Q}}(1) \otimes p^{*} \mathcal{O}_{X}(-1) \rightarrow 0
\end{aligned}
$$

we see that $\mathcal{H}^{0}\left(p_{*} q^{*} K\right) \cong \mathcal{O}_{X}(-1), \mathcal{H}^{1}\left(p_{*} q^{*} K\right) \cong \mathcal{U}_{X}$ and $p_{*} q^{*} T_{\mathbb{P}(W)}(-2)_{\mid \mathcal{Q}} \cong \mathcal{O}_{X}(-1)$, whereof we deduce $p_{*} q^{*} S_{\mathcal{Q}} \cong \mathcal{U}_{X}$. Thus $M_{\mid X} \cong\left(\tilde{p}_{*} \tilde{q}^{*} S_{\mathcal{Q}}\right)_{\mid X} \cong p_{*}\left(\left(\tilde{q}^{*} S_{\mathcal{Q}}\right)_{\mid \mathbb{P}_{X}\left(\mathcal{U}_{X}\right)}\right) \cong p_{*} q^{*} S_{\mathcal{Q}} \cong \mathcal{U}_{X}$.

Finally, we note that (58) implies that $\mathbb{P}_{X}\left(\mathcal{U}_{X}\right)=\mathbb{P}_{\mathcal{Q}}(K)$ is the zero locus of a section of the bundle $\tilde{q}^{*} S_{\mathcal{Q}} \otimes \tilde{p}^{*} \mathcal{O}_{\mathrm{OGr}(2, W)}(1)$ on $\mathbb{P}_{\mathcal{Q}}\left(T_{\mathbb{P}(W)}(-2)_{\mid \mathcal{Q}}\right) \subset \mathbb{P}_{\mathbb{P}(W)}\left(T_{\mathbb{P}(W)}(-2)\right)=\mathrm{FI}(1,2 ; W)$. Restricting to $\mathrm{OF}(1,2 ; W)=\mathbb{P}_{\mathrm{OGr}(2, W)}\left(\mathcal{U}_{\mathrm{OGr}}\right)$ we see that $\mathbb{P}_{X}\left(\mathcal{U}_{X}\right)$ is the zero locus of a section of the bundle $\tilde{q}^{*} S_{\mathcal{Q}} \otimes$ $\tilde{p}^{*} \mathcal{O}_{\mathrm{OGr}(2, W)}(1)$, hence $X$ is the zero locus of a section of the vector bundle $\tilde{p}_{*}\left(\tilde{q}^{*} S_{\mathcal{Q}} \otimes \tilde{p}^{*} \mathcal{O}_{\mathrm{OGr}(2, W)}(1)\right)=$ $M \otimes \mathcal{O}_{\mathrm{OGr}(2, W)}(1) \cong M^{*}$. Therefore, its structure sheaf admits a Koszul resolution which takes form of the exact sequence of the lemma.

Consider $X \times \operatorname{OGr}(2, W)$ and the subscheme $\Sigma^{\prime} \subset X \times \operatorname{OGr}(2, W) \subset \operatorname{Gr}(2, W) \times \operatorname{Gr}(2, W)$ of pairs of intersecting two-dimensional subspaces. 
Lemma B.4. The following sequences on $X \times \operatorname{OGr}(2, W)$ are exact

$$
\begin{aligned}
0 \rightarrow & \mathcal{U}_{X}(-1) \otimes \mathcal{O}_{\mathrm{OGr}(2, W)}(-1) \rightarrow \mathcal{O}_{X}(-1) \otimes\left(W / \mathcal{U}_{\mathrm{OG}}\right)(-1) \rightarrow \mathcal{U}_{X} \otimes M \rightarrow \mathcal{O}_{X} \otimes \mathcal{O}_{\mathrm{OGr}(2, W)} \rightarrow \mathcal{O}_{\Sigma^{\prime}} \rightarrow 0 . \\
& \rightarrow \mathcal{O}_{X}(-1) \otimes \mathcal{O}_{\mathrm{OGr}(2, W)}(-1) \rightarrow \mathcal{U}_{X}^{*}(-1) \otimes M \rightarrow \mathcal{O}_{X} \otimes \mathcal{U}_{\mathrm{OGr}}^{\perp} \rightarrow \mathcal{U}_{X}^{*} \otimes \mathcal{O}_{\mathrm{OGr}(2, W)} \rightarrow \mathcal{F}_{\Sigma^{\prime}} \rightarrow 0 .
\end{aligned}
$$

where $\mathcal{F}_{\Sigma^{\prime}}=\mathcal{E} x t^{3}\left(\mathcal{O}_{\Sigma^{\prime}}, \mathcal{O}_{X}(-1) \otimes \mathcal{O}_{\mathrm{OGr}(2, W)}(-1)\right)$.

Proof: Consider the decomposition with respect to the exceptional collection

$$
\left\langle\mathcal{O}_{\mathcal{Q}}(-3), \mathcal{O}_{\mathcal{Q}}(-2), \mathcal{S}_{\mathcal{Q}}(-1), \mathcal{O}_{\mathcal{Q}}(-1), \mathcal{O}_{\mathcal{Q}}, \mathcal{O}_{\mathcal{Q}}(1)\right\rangle=\mathcal{D}^{b}(\mathcal{Q})
$$

of the structure sheaf of the variety $\operatorname{OF}(1,2 ; W) \subset \operatorname{OGr}(2, W) \times \mathcal{Q}$. It is easy to check that the decomposition takes form

$$
\begin{aligned}
0 \rightarrow \mathcal{O}_{\mathrm{OGr}(2, W)}(-1) \otimes \mathcal{O}_{\mathcal{Q}}(-3) \rightarrow\left(W / \mathcal{U}_{\mathrm{OGr}}\right)(-1) \otimes \mathcal{O}_{\mathcal{Q}}(-2) & \rightarrow M \otimes \mathcal{S}_{\mathcal{Q}}(-1) \rightarrow \\
& \rightarrow \mathcal{U}_{\mathrm{OGr}}^{\perp} \otimes \mathcal{O}_{\mathcal{Q}}(-1) \rightarrow \mathcal{O}_{\mathrm{OGr}(2, W)} \otimes \mathcal{O}_{\mathcal{Q}} \rightarrow \mathcal{O}_{\mathrm{OF}(1,2 ; W)} \rightarrow 0 .
\end{aligned}
$$

Now consider the product $\operatorname{OGr}(2, W) \times \mathcal{Q} \times \operatorname{OGr}(2, W)$ and the subvariety

$$
\Sigma^{\prime \prime}=\operatorname{OF}(1,2 ; W) \times{ }_{\mathcal{Q}} \mathrm{OF}(1,2 ; W) \subset \mathrm{OGr}(2, W) \times \mathcal{Q} \times \operatorname{OGr}(2, W) .
$$

It is clear that $\Sigma^{\prime \prime \prime}=p_{13}\left(\Sigma^{\prime \prime}\right) \subset \operatorname{OGr}(2, W) \times \operatorname{OGr}(2, W)$ is just the subscheme of pairs of intersecting twodimensional subspaces. Tensoring the pullbacks of the resolutions (59) via the projections $\operatorname{OGr}(2, W) \times$ $\mathcal{Q} \times \operatorname{OGr}(2, W) \rightarrow \operatorname{OGr}(2, W) \times \mathcal{Q}$ and $\operatorname{OGr}(2, W) \times \mathcal{Q} \times \operatorname{OGr}(2, W) \rightarrow \mathcal{Q} \times \operatorname{OGr}(2, W)$, and applying $p_{13 *}$ we obtain

$$
\begin{aligned}
0 \rightarrow \mathcal{U}_{\mathrm{OGr}}(-1) \otimes \mathcal{O}_{\mathrm{OGr}(2, W)}(-1) \rightarrow \mathcal{O}_{\mathrm{OGr}(2, W)}(-1) & \otimes\left(W / \mathcal{U}_{\mathrm{OGr}}\right)(-1) \rightarrow \\
& \rightarrow M \otimes M \rightarrow \mathcal{O}_{\mathrm{OGr}(2, W)} \otimes \mathcal{O}_{\mathrm{OGr}(2, W)} \rightarrow \mathcal{O}_{\Sigma^{\prime \prime \prime}} \rightarrow 0 .
\end{aligned}
$$

Restricting to $X \times \operatorname{OGr}(2, W)$ and taking into account that $\mathcal{U}_{\mathrm{OGr} \mid X} \cong M_{\mid X} \cong \mathcal{U}_{X}$, we obtain the first sequence. Applying the functor RHom $\left(-, \mathcal{O}_{X}(-1) \otimes \mathcal{O}_{\mathrm{OGr}(2, W)}(-1)\right)$ we obtain the second sequence.

Consider the truncated first sequence of lemma B.4 as a resolution of the sheaf of ideals $J_{\Sigma^{\prime}}$. Tensoring it with $\mathcal{O}_{X}(1)$ and pushing forward via the projection $p_{2}: X \times \operatorname{OGr}(2, W) \rightarrow \operatorname{OGr}(2, W)$ we obtain exact sequence

$$
0 \rightarrow\left(W / \mathcal{U}_{\mathrm{OGr}}\right)(-1) \rightarrow W^{*} \otimes M \rightarrow p_{2 *}\left(J_{\Sigma^{\prime}} \otimes \mathcal{O}_{X}(1)\right) \rightarrow 0 .
$$

Lemma B.5. $p_{2 *}\left(J_{\Sigma^{\prime}} \otimes \mathcal{O}_{X}(1)\right)$ is a vector bundle of rank 9 on $\operatorname{OGr}(2, W)$. Moreover, its restriction to $X$ is isomorphic to the bundle $\mathfrak{p}$ on $X=G_{2} / P$ (here $P$ is a parabolic subgroup of $G_{2}$ and $\mathfrak{p}$ is the corresponding parabolic Lie subalgebra).

Proof: For the first claim it suffices to check that $p_{2 *}\left(\mathcal{O}_{\Sigma^{\prime}} \otimes \mathcal{O}_{X}(1)\right)$ is a vector bundle. But it is easy to see that the fiber of $\Sigma^{\prime}$ over a point $u \in \operatorname{OGr}(2, W)$ is either a Hirzebruch surface $F_{1}$ (if $u \notin X$ ), or a cone over a rational twisted cubic curve (if $u \in X)$. In both cases $H^{0}\left(\Sigma_{u}^{\prime}, \mathcal{O}_{X}(1)\right)$ is 5-dimensional, so $p_{2 *}\left(\mathcal{O}_{\Sigma^{\prime}} \otimes \mathcal{O}_{X}(1)\right)$ is a vector bundle of rank 5 and the first claim follows. Moreover, restricting this bundle to $X$ we obtain a $G_{2}$-equivariant rank 5 quotient bundle of the bundle $V^{*} \otimes \mathcal{O}_{X} \cong \mathfrak{g} \otimes \mathcal{O}_{X}$. But the only such bundle is the tangent bundle $T_{X} \cong \mathfrak{g} / \mathfrak{p}$, hence $p_{2 *}\left(J_{\Sigma^{\prime}} \otimes \mathcal{O}_{X}(1)\right)_{\mid X} \cong \mathfrak{p}$.

Consider the projectivization

$$
\widetilde{Y}=\mathbb{P}_{\mathrm{OGr}(2, W)}\left(p_{2 *}\left(J_{\Sigma^{\prime}} \otimes \mathcal{O}_{X}(1)\right)\right) .
$$

The natural embedding $p_{2 *}\left(J_{\Sigma^{\prime}} \otimes \mathcal{O}_{X}(1)\right) \rightarrow H^{0}\left(X, \mathcal{O}_{X}(1)\right) \otimes \mathcal{O}_{\mathrm{OGr}(2, W)}=V^{*} \otimes \mathcal{O}_{\mathrm{OGr}(2, W)}$ induces a morphism $\tilde{Y} \stackrel{\tilde{g}}{\longrightarrow} \mathbb{P}\left(V^{*}\right)$. Let $\pi: \tilde{Y} \rightarrow \operatorname{OGr}(2, W)$ denote the canonical projection. So we have

$$
\mathcal{O}_{\widetilde{Y}}(H):=\tilde{g}^{*} \mathcal{O}_{\mathbb{P}\left(V^{*}\right)}(1), \quad \tilde{g}: \widetilde{Y} \rightarrow \mathbb{P}\left(V^{*}\right), \quad \text { and } \mathcal{O}_{\tilde{Y}}(h):=\pi^{*} \mathcal{O}_{\mathrm{OGr}(2, W)}(1), \quad \pi: \tilde{Y} \rightarrow \operatorname{OGr}(2, W) .
$$


Consider the product $X \times \tilde{Y}$, the projection $\operatorname{id}_{X} \times \pi: X \times \tilde{Y} \rightarrow X \times \operatorname{OGr}(2, W)$, the subschemes

$$
\Sigma=\left(\operatorname{id}_{X} \times \pi\right)^{-1}\left(\Sigma^{\prime}\right), \quad \tilde{Q}=Q(X, \tilde{Y})=(X \times \tilde{Y}) \times \mathbb{P}(V) \times \mathbb{P}\left(V^{*}\right) Q,
$$

and the equation $s_{\tilde{Q}} \in H^{0}\left(X \times \tilde{Y}, \mathcal{O}_{X}(1) \otimes \mathcal{O}_{\widetilde{Y}}(H)\right)=H^{0}\left(X \times \tilde{Y},(f \times \tilde{g})^{*} \mathcal{O}_{\mathbb{P}(V) \times \mathbb{P}\left(V^{*}\right)}(1,1)\right) \cong V^{*} \otimes V$ of $\tilde{Q}$ in $X \times \tilde{Y}$.

Lemma B.6. The section $s_{\tilde{Q}}$ vanishes on the scheme $\Sigma$.

Consider the pullback of the second exact sequence of lemma B.4 to $X \times \tilde{Y}$. Consider the cone $G^{\prime}$ of its first morphism twisted by $\mathcal{O}_{X}(1) \otimes \mathcal{O}_{\widetilde{Y}}(H)$, and the cone $G^{\prime \prime}$ of its third morphism as objects of $\mathcal{D}^{b}(X \times \tilde{Y})$, so that we have exact triangles

$$
\begin{gathered}
E_{1} \otimes \mathcal{O}_{\widetilde{Y}}(H-h) \stackrel{\tilde{e}^{\prime}}{\longrightarrow} E_{2} \otimes M(H) \longrightarrow G^{\prime} \\
E_{1} \otimes \mathcal{U}_{\mathrm{OGr}}^{\perp} \stackrel{\tilde{e}^{\prime \prime}}{\longrightarrow} E_{2} \otimes \mathcal{O}_{\widetilde{Y}} \longrightarrow G^{\prime \prime}
\end{gathered}
$$

Then the pullback of the second exact sequence of lemma B.4 can be rewritten as exact triangle

$$
G^{\prime} \otimes\left(\mathcal{O}_{X}(-1) \otimes \mathcal{O}_{\widetilde{Y}}(-H)\right)[1] \rightarrow G^{\prime \prime} \rightarrow \mathcal{F}_{\Sigma} .
$$

It is clear that morphisms $\tilde{e}^{\prime}$ and $\tilde{e}^{\prime \prime}$ determine a structure of Q-representations on families $\left(\mathcal{O}_{\tilde{Y}}(H-\right.$ $h), M(H))$ and $\left(\mathcal{U}_{\mathrm{OGr}}^{\perp}, \mathcal{O}_{\widetilde{Y}}\right)$ on $\tilde{Y}$.

Lemma B.7. There exist a unique extension

$$
0 \rightarrow\left(\mathcal{O}_{\widetilde{Y}}(H-h), M(H)\right) \rightarrow\left(\tilde{F}_{1}, \tilde{F}_{2}\right) \rightarrow\left(\mathcal{U}_{\mathrm{OGr}}^{\perp}, \mathcal{O}_{\widetilde{Y}}\right) \rightarrow 0
$$

in the category of families of Q-representations on $\widetilde{Y}$ such that the cone of the induced morphism $\tilde{e}$ : $E_{1} \otimes \tilde{F}_{1} \rightarrow E_{2} \otimes \tilde{F}_{2}$ is a sheaf supported at $\tilde{Q}$.

Lemma B.7 says that the family $\left(\tilde{F}_{1}, \tilde{F}_{2}\right)$ on $\tilde{Y}$ is a family of Q-representations and that on $X \times \tilde{Y}$ we have the following exact sequence:

$$
0 \rightarrow E_{1} \otimes \tilde{F}_{1} \stackrel{\tilde{e}}{\longrightarrow} E_{2} \otimes \tilde{F}_{2} \rightarrow \tilde{\imath}_{*} \tilde{\mathcal{E}} \rightarrow 0,
$$

where $\tilde{\mathcal{E}}$ is a coherent sheaf on $\tilde{Q}$.

Lemma B.8. The family $\left(\tilde{F}_{1}, \tilde{F}_{2}\right)$ induces a morphism $\phi: \widetilde{Y} \rightarrow \bar{Y}_{3}$ such that $\tilde{g}=\bar{g}_{3} \circ \phi$.

Let $\tilde{Y}^{\circ}=\tilde{Y} \backslash \tilde{g}^{-1}\left(Z_{1}\right)$ and denote the restriction of $\tilde{g}$ and $\phi$ to $\tilde{Y}^{\circ}$ by the same symbols.

Recall that we have a sheaf of Azumaya algebras $\mathcal{A}_{3}$ on $Y_{3}$ and that $F_{2}$ is a locally projective $\mathcal{A}_{3}$-module on $Y_{3}$ of rank 3 . Let $\mathbb{P}_{Y_{3}, \mathcal{A}_{3}}\left(F_{2}^{*}\right)$ denote the moduli space of locally projective quotient $\mathcal{A}_{3}$-modules of rank 1. Then $\mathbb{P}_{Y_{2}, \mathcal{A}_{2}}\left(F_{2}^{*}\right)$ is a twisted $\mathbb{P}^{2}$-bundle over $Y_{3}$.

Proposition B.9. We have $\tilde{Y}^{\circ}=\mathbb{P}_{Y_{3}, \mathcal{A}_{3}}\left(F_{2}^{*}\right)$.

Proof: The map $\tilde{Y}^{\circ} \rightarrow \mathbb{P}_{Y_{3}, \mathcal{A}_{3}}\left(F_{2}^{*}\right)$ can be constructed like in proposition A.9. Thus it remains to construct the inverse map. Choose a point $\tilde{y} \in \mathbb{P}_{Y_{3}, \mathcal{A}_{3}}\left(F_{2}^{*}\right)$. Let $\rho: W \otimes R_{1} \rightarrow R_{2}$ be the corresponding (6,3)-dimensional representation of the quiver $\mathrm{Q}$, and let $R_{2} \rightarrow \mathrm{k}$ be the corresponding one-dimensional quotient. Let $R_{2}^{\prime} \subset R_{2}$ be its kernel and consider the composition $E_{1} \otimes R_{1} \stackrel{e_{\rho}}{\longrightarrow} E_{2} \otimes R_{2} \rightarrow E_{2}$. Taking $\operatorname{Hom}\left(E_{1},-\right)$ we obtain a map $R_{1} \rightarrow W^{*}$. Let $R_{1}^{\prime}$ denote the kernel of this map. Then we have a diagram (54) and an exact sequence (55) Note that the sheaf $C$ is supported on the hyperplane section $X_{y}=H_{g_{3}(y)} \cap X \subset X$ and its rank at the generic point of $X_{y}$ equals $c_{1}\left(E_{2} \otimes R_{2}\right)-c_{1}\left(E_{1} \otimes R_{1}\right)=3$.

We claim that $\operatorname{dim} R^{\prime}=1$ and we are going to prove it by a case by case analysis. 
Since $\left(R_{1}^{\prime}, R_{2}^{\prime}\right)$ is a subrepresentation of $\left(R_{1}, R_{2}\right)$ and $\left(R_{1}, R_{2}\right)$ is stable by lemma 5.5 we have

$$
\operatorname{dim} R_{1}^{\prime}<4
$$

so we need to reject three cases: $\operatorname{dim} R_{1}^{\prime}=3, \operatorname{dim} R_{1}^{\prime}=2$ and $\operatorname{dim} R_{1}^{\prime}=0$.

The cases $\operatorname{dim} R_{1}^{\prime}=2$ and $\operatorname{dim} R_{1}^{\prime}=3$ are treated along the same lines as the case $\operatorname{dim} R_{1}^{\prime}=2$ in the proof of proposition A.9. The only difference is that in the case $\operatorname{dim} R_{1}^{\prime}=2$ the sheaf $T\left(C^{\prime}\right)$ can have rank 1 at the generic point of $X_{y}$, but it is easy to see that this is possible only in the case $C^{\prime} \cong \mathcal{U}_{X}^{*} \oplus \mathcal{U}_{X}^{*} /\left(\mathcal{O}_{X} \oplus \mathcal{O}_{X}\right)$, which means that $g_{3}(y) \in Z_{1}$.

Assume that $R_{1}^{\prime}=0$. Then the map $R_{1} \rightarrow W^{*}$ is injective, so we can consider $R_{1}$ as a hyperplane in $\mathbb{P}\left(W^{*}\right)$, or equivalently as a point $w_{y} \in \mathbb{P}(W)$. If the cokernel of the morphism $K^{\prime \prime} \rightarrow C^{\prime}=\mathcal{U}_{X}^{*} \oplus \mathcal{U}_{X}^{*}$ is supported scheme-theoretically on a hyperplane section $X_{y} \subset X$, then the morphism $\mathcal{U}_{X} \oplus \mathcal{U}_{X} \cong$ $\left(\mathcal{U}_{X}^{*} \oplus \mathcal{U}_{X}^{*}\right) \otimes \mathcal{O}_{X}(-1) \rightarrow \mathcal{U}_{X}^{*} \oplus \mathcal{U}_{X}^{*}$ factors through $K^{\prime \prime}$ and the cokernel of the corresponding morphism $\mathcal{U}_{X} \oplus \mathcal{U}_{X} \rightarrow K^{\prime \prime}$ is supported on the same hyperplane section $X_{y} \subset X$. On the other hand, it is easy to compute that $\operatorname{dim} \operatorname{Hom}\left(\mathcal{U}_{X}, K^{\prime \prime}\right)=1$ for $w_{y} \notin \mathcal{Q}$, and $\operatorname{dim} \operatorname{Hom}\left(\mathcal{U}_{X}, K^{\prime \prime}\right)=4$ for $w_{y} \in \mathcal{Q}$. Therefore we must have $w_{y} \in \mathcal{Q}$. Moreover, it is clear that the dimension of the set of such hyperplane sections $X_{y} \subset X$ has dimension less than $\operatorname{dim} \mathcal{Q}+\operatorname{dim} \operatorname{Gr}(2,4)=5+4=9$. On the other hand, the set of such hyperplane sections is evidently $G_{2}$-invariant in $\mathbb{P}\left(V^{*}\right)$, and it follows from the description of all $G_{2}$-invariant closed subsets of $\mathbb{P}\left(V^{*}\right)$ that this set is contained in $Z_{1}$.

Thus we have shown that if $g_{3}(y) \notin Z_{1}$ then $\operatorname{dim} R_{1}^{\prime}=1, \operatorname{dim} R_{1} / R_{1}^{\prime}=5$. The top and the bottom lines of the diagram (54) take form

$$
\begin{gathered}
0 \rightarrow \mathcal{O}_{X} \stackrel{e^{\prime}}{\rightarrow} \mathcal{U}_{X}^{*} \oplus \mathcal{U}_{X}^{*} \rightarrow C^{\prime} \rightarrow 0, \\
0 \rightarrow K^{\prime \prime} \rightarrow \mathcal{O}_{X}^{\oplus 5} \stackrel{e^{\prime \prime}}{\rightarrow} \mathcal{U}_{X}^{*} \rightarrow C^{\prime \prime} \rightarrow 0 .
\end{gathered}
$$

The map $e^{\prime \prime}$ is given by a 5 -dimensional subspace of $W^{*}$, that is by a point $u \in \operatorname{Gr}(2, W)$. The same arguments as in the proof of proposition A.9 show that we can glue the sequence (63) twisted by $\mathcal{O}_{X}(-1)$ with the sequence (64) into the following exact sequence

$$
0 \rightarrow \mathcal{O}_{X}(-1) \stackrel{e^{\prime}}{\longrightarrow} \mathcal{U}_{X}^{*}(-1) \oplus \mathcal{U}_{X}^{*}(-1) \rightarrow \mathcal{O}_{X}^{\oplus 5} \stackrel{u}{\longrightarrow} \mathcal{U}_{X}^{*} \rightarrow C^{\prime \prime} \rightarrow 0 .
$$

Then it is easy to see that this sequence must coincide with the second sequence of lemma B.4 restricted to a fiber of $X \times \operatorname{OGr}(2, W)$ over a point of $\operatorname{OGr}(2, W)$. In particular, $u \in \operatorname{OGr}(2, W)$. Associating to the point $\tilde{y}$ the point $u \in \operatorname{OGr}(2, W)$ we obtain a map $\mathbb{P}_{Y_{3}, \mathcal{A}_{3}}\left(F_{2}^{*}\right) \rightarrow \operatorname{OGr}(2, W)$. On the other hand, the support hyperplane $X_{y}$ of $C$ contains $\operatorname{supp}\left(C^{\prime \prime}\right)=\Sigma_{u}^{\prime}$, therefore the map

$$
\mathbb{P}_{Y_{3}, \mathcal{A}_{3}}\left(F_{2}^{*}\right) \rightarrow \operatorname{OGr}(2, W) \times \mathbb{P}\left(V^{*}\right), \quad \tilde{y} \mapsto\left(u, g_{3}(y)\right)
$$

factors through $\tilde{Y}=\mathbb{P}_{\mathrm{OGr}(2, W)}\left(p_{2 *}\left(J_{\Sigma^{\prime}} \otimes \mathcal{O}_{X}(1)\right)\right) \subset \mathrm{OGr}(2, W) \times \mathbb{P}\left(V^{*}\right)$. Moreover, it is clear that its image lies in $\widetilde{Y}^{\circ}$, so we have constructed a map $\mathbb{P}_{Y_{3}, \mathcal{A}_{3}}\left(F_{2}^{*}\right) \rightarrow \widetilde{Y}^{\circ}$.

It remains to note that the constructed maps are mutually inverse.

Theorem B.10. The map $g_{3}: Y_{3} \rightarrow \mathbb{P}\left(V^{*}\right) \backslash Z_{1}$ is a double covering.

Proof: Since $\tilde{Y}^{\circ}$ is smooth it follows from proposition B.9 that $Y_{3}$ is smooth and moreover $\operatorname{dim} Y_{3}=$ $\operatorname{dim} \tilde{Y}^{\circ}-2=13$. On the other hand, since $\tilde{g}=\bar{g}_{3} \circ \phi, \tilde{g}$ and $\phi$ are proper and dominant, it follows that $g_{3}$ is a proper dominant map $Y_{3} \rightarrow \mathbb{P}\left(V^{*}\right) \backslash Z_{1}$. Since $Y_{3}$ and $\mathbb{P}\left(V^{*}\right) \backslash Z_{1}$ are smooth varieties of equal dimension and $\operatorname{rank} \operatorname{Pic} Y_{3}=\operatorname{rank} \operatorname{Pic} \widetilde{Y}^{\circ}-1=1=\operatorname{rank} \operatorname{Pic}\left(\mathbb{P}\left(V^{*}\right) \backslash Z_{1}\right)$ it follows that $g_{3}$ is a finite covering. It remains to show that $\operatorname{deg} g_{3}=2$. This can be done by a direct calculation using the intersection theory of $\operatorname{OGr}(2, W)$. Alternatively, we can argue as follows. 
Consider the fiber product $\tilde{Y}^{\circ} \times{ }_{\mathrm{OGr}(2, W)} X$. By lemma B.5 we have $\tilde{Y}^{\circ} \times{ }_{\mathrm{OGr}(2, W)} X$ is an open subset of $\mathbb{P}_{X}(\mathfrak{p})$, so and it is clear that the map $\tilde{g}_{\mid \mathbb{P}_{X}(\mathfrak{p})}: \mathbb{P}_{X}(\mathfrak{p}) \rightarrow \mathbb{P}\left(V^{*}\right)=\mathbb{P}(\mathfrak{g})$ is the projectivization of the partial Springer-Grothendieck map $\operatorname{Tot}(\mathfrak{p}) \rightarrow \mathfrak{g}$. Therefore, by $[\mathrm{Hu}]$ its degree equals $12 / 2=6$. On the other hand, since $X \subset \operatorname{OGr}(2, W)$ is the zero locus of a section of the vector bundle $M^{*}$ and the restriction of $M$ to the fiber $\mathbb{P}^{2}$ of $\widetilde{Y}^{\circ}$ over $Y_{3}$ is isomorphic to $\Omega_{\mathbb{P}^{2}}$ by (62) we see that the degree of the $\operatorname{map} \phi_{\mid \mathbb{P}_{X}(\mathfrak{p})}: \mathbb{P}_{X}(\mathfrak{p}) \rightarrow Y_{3}$ equals $c_{2}\left(T_{\mathbb{P}^{2}}\right)=3$. Hence $\operatorname{deg} g_{3}=6 / 3=2$.

Corollary B.11. If the data (D.3)-(D.5) is given by $Y=Y_{3}, \mathcal{A}_{Y}=\mathcal{A}_{3}, \mathbf{Z}=\bar{Z}_{3}=Z_{1}, g=g_{3}$, with $\left(F_{1}, F_{2}\right)$ being the universal family, and with $\phi$ being induced by the Q-representation structure on $\left(F_{1}, F_{2}\right)$, then all conditions (C.1)-(C.8) are satisfied. Moreover, the branching locus of the map $g: Y \rightarrow \mathbb{P}\left(V^{*}\right) \backslash \mathbf{Z}$ is the hypersurface $X^{\vee} \backslash \mathbf{Z}$, where $X^{\vee} \subset \mathbb{P}\left(V^{*}\right)$ is the projectively dual sextic hypersurface.

Proof: Since the conditions (C.1)-(C.3), (45) and (51) are satisfied by lemma B.1, it follows from theorem 5.7 that the conditions (C.4)-(C.6) are true. On the other hand, (C.8) is true because $\mathbf{Z}=$ $\mu(\operatorname{Gr}(2, W))$ by lemma B.2 and $\operatorname{dim} \mathbf{Z} \cap H=\operatorname{dim} \operatorname{Gr}(2, W)-1=9$, while $N-i-2=14-3-2=9$. Finally, by theorem B.10 the map $Y=Y_{3} \rightarrow \mathbb{P}\left(V^{*}\right)$ is a double covering and $\operatorname{codim}_{Y \times Y}\left(Y \times \mathbb{P}\left(V^{*}\right) Y\right)=$ $\operatorname{dim} Y=\operatorname{dim} \mathbb{P}\left(V^{*}\right)=13$, hence $\operatorname{dim} X+N-\operatorname{codim}_{Y \times Y}\left(Y \times_{\mathbb{P}\left(V^{*}\right)} Y\right)=5+14-13=6=2 i$, and (C.7) is true.

Finally, we note that the branching locus of $g$ is nothing but the singular locus of $g$, hence it coincides with $X^{\vee} \backslash \mathbf{Z}$ by corollary 4.30 .

\section{Appendix C. Intersection of QUADRICS IN $\mathbb{P}^{5}$}

In this appendix we show that a double covering of $\mathbb{P}^{20}$ ramified in a sextic hypersurface is homologically projectively dual to the double Veronese subvariety $\mathbb{P}^{5} \subset \mathbb{P}^{20}$.

Recall the notation. Let $W=\mathrm{k}^{6}$ and

$$
X=\mathbb{P}(W)=\mathbb{P}^{5}, \quad\left(E_{1}, E_{2}\right)=\left(\mathcal{O}_{X}, \mathcal{O}_{X}(1)\right) .
$$

Let $V=S^{2} W=\mathrm{k}^{21}$ and let $f: X \rightarrow \mathbb{P}(V)$ be the double Veronese embedding.

Lemma C.1. We have $\operatorname{dim} X=5, \omega_{X} \cong \mathcal{O}_{X}(-6) \cong f^{*} \mathcal{O}_{\mathbb{P}(V)}(-3)$. Moreover, conditions (C.1)-(C.3) as well as the additional conditions (45) and (51) are satisfied for $X$.

Now we are going to apply the construction of section 5 and to describe the space $Y=Y_{4}$ explicitly. We use the same approach as in Appendix A. The arguments in most cases are the same. So we will not repeat them, but describe the differences.

Instead of $Y_{4}$ we will describe $\mathbb{P}_{Y_{4}, \mathcal{A}_{4}}\left(F_{2}^{*}\right)$, the moduli space of quotient $\mathcal{A}_{4}$-modules of rank 1 . The fiber of $\mathbb{P}_{Y_{4}, \mathcal{A}_{4}}\left(F_{2}^{*}\right)$ over a point of $Y_{4}$ corresponding to a $(4,4)$-dimensional representation $\rho=\left(R_{1}, R_{2}\right)$ of the quiver $\mathrm{Q}$ coincides with $\mathbb{P}\left(R_{2}^{*}\right)$. We show that any hyperplane $R_{2}^{\prime} \subset R_{2}$ can be extended in a unique way to a $(1,3)$-dimensional subrepresentation $\rho^{\prime}=\left(R_{1}^{\prime}, R_{2}^{\prime}\right)$. The moduli space of $(1,3)$-dimensional representations of $\mathrm{Q}$ is $\operatorname{Gr}(3, W)$. We claim that defined in this way map $\mathbb{P}_{Y_{4}, \mathcal{A}_{4}}\left(F_{2}^{*}\right) \rightarrow \operatorname{Gr}(3, W)$ identifies $\mathbb{P}_{Y_{4}, \mathcal{A}_{4}}\left(F_{2}^{*}\right)$ with an open subset in a $\mathbb{P}^{14}$-bundle over $\operatorname{Gr}\left(3, W^{*}\right)$.

Lemma C.2. We have $Z_{2} \subset \mathbb{P}\left(V^{*}\right)$ is the locus of rank 4 quadrics and $\cup_{0<D<\mathcal{H}} Z^{|D|} \subset \mathbb{P}\left(V^{*}\right)$ is the locus of rank 2 quadrics.

Consider the product $X \times \operatorname{Gr}(3, W)$ and the subscheme $\Sigma^{\prime}=\operatorname{Fl}(1,3 ; W) \subset X \times \operatorname{Gr}(3, W)$.

Lemma C.3. The following sequence on $X \times \operatorname{Gr}(3, W)$ is exact

$$
0 \rightarrow \mathcal{O}_{X}(-3) \otimes \mathcal{O}_{\mathrm{Gr}(3, W)}(-1) \rightarrow \mathcal{O}_{X}(-2) \otimes \Lambda^{2} \mathcal{U}_{\mathrm{Gr}(3, W)}^{\perp} \rightarrow \mathcal{O}_{X}(-1) \otimes \mathcal{U}_{\mathrm{Gr}(3, W)}^{\perp} \rightarrow \mathcal{O}_{X} \otimes \mathcal{O}_{\mathrm{Gr}(3, W)} \rightarrow \mathcal{O}_{\Sigma^{\prime}} \rightarrow 0
$$


Proof: This is just the Koszul resolution of $\Sigma^{\prime}$.

Consider the truncated sequence of lemma C.3 as a resolution of the sheaf of ideals $J_{\Sigma^{\prime}}$. Tensoring it with $\mathcal{O}_{X}(2)=f^{*} \mathcal{O}_{\mathbb{P}(V)}(1)$ and pushing forward via the projection $X \times \operatorname{Gr}(3, W) \rightarrow \operatorname{Gr}(3, W)$ we obtain exact sequence

$$
0 \rightarrow \Lambda^{2} \mathcal{U}_{\mathrm{Gr}(3, W)}^{\perp} \rightarrow W^{*} \otimes \mathcal{U}_{\mathrm{Gr}(3, W)}^{\perp} \rightarrow p_{2 *}\left(J_{\Sigma^{\prime}} \otimes \mathcal{O}_{X}(2)\right) \rightarrow 0 .
$$

Lemma C.4. $p_{2 *}\left(J_{\Sigma^{\prime}} \otimes \mathcal{O}_{X}(1)\right)$ is a vector bundle of rank 15 on $\operatorname{Gr}(3, W)$. Moreover, its restriction to $\operatorname{LGr}(3, W) \subset \operatorname{Gr}(3, W)$ is isomorphic to the bundle $\mathfrak{p}$ on $\operatorname{LGr}(3, W)=\operatorname{Sp}(W) / P$ (here $P$ is a parabolic subgroup of $\mathrm{Sp}(W)$ and $\mathfrak{p}$ is the corresponding parabolic Lie subalgebra).

Proof: It is clear that the morphism $\Lambda^{2} \mathcal{U}_{\mathrm{Gr}(3, W)}^{\perp} \rightarrow W^{*} \otimes \mathcal{U}_{\mathrm{Gr}(3, W)}^{\perp}$ is an embedding of vector bundles. Moreover, restricting this bundle to $\operatorname{LGr}(3, W)$ we obtain a $\operatorname{Sp}(W)$-equivariant rank 15 subbundle of the bundle $V^{*} \otimes \mathcal{O}_{X} \cong \mathfrak{g} \otimes \mathcal{O}_{X}$. But the only such bundle is the bundle $\mathfrak{p}$.

Consider the projectivization

$$
\widetilde{Y}=\mathbb{P}_{\operatorname{Gr}(3, W)}\left(p_{2 *}\left(J_{\Sigma^{\prime}} \otimes \mathcal{O}_{X}(2)\right)\right) .
$$

The natural embedding $p_{2 *}\left(J_{\Sigma^{\prime}} \otimes \mathcal{O}_{X}(2)\right) \rightarrow H^{0}\left(X, \mathcal{O}_{X}(2)\right) \otimes \mathcal{O}_{\mathrm{Gr}(3, W)}=V^{*} \otimes \mathcal{O}_{\mathrm{Gr}(3, W)}$ induces a morphism $\tilde{Y} \stackrel{\tilde{g}}{\longrightarrow} \mathbb{P}\left(V^{*}\right)$. Let $\pi: \widetilde{Y} \rightarrow \operatorname{Gr}(3, W)$ denote the canonical projection. So we have

$$
\mathcal{O}_{\widetilde{Y}}(H)=\tilde{g}^{*} \mathcal{O}_{\mathbb{P}\left(V^{*}\right)}(1), \quad \tilde{g}: \tilde{Y} \rightarrow \mathbb{P}\left(V^{*}\right), \quad \text { and } \quad \mathcal{O}_{\widetilde{Y}}(h)=\pi^{*} \mathcal{O}_{\operatorname{Gr}(3, W)}(1), \quad \pi: \tilde{Y} \rightarrow \operatorname{Gr}(3, W) .
$$

Consider the product $X \times \tilde{Y}$, the projection $\mathrm{id}_{X} \times \pi: X \times \widetilde{Y} \rightarrow X \times \operatorname{Gr}(3, W)$, the subschemes

$$
\Sigma=\left(\operatorname{id}_{X} \times \pi\right)^{-1}\left(\Sigma^{\prime}\right), \quad \tilde{Q}=Q(X, \tilde{Y})=(X \times \tilde{Y}) \times_{\mathbb{P}(V) \times \mathbb{P}\left(V^{*}\right)} Q,
$$

and the equation $s_{\tilde{Q}} \in H^{0}\left(X \times \tilde{Y}, \mathcal{O}_{X}(2) \otimes \mathcal{O}_{\tilde{Y}}(H)\right)=H^{0}\left(X \times \tilde{Y},(f \times \tilde{g})^{*} \mathcal{O}_{\mathbb{P}(V) \times \mathbb{P}\left(V^{*}\right)}(1,1)\right) \cong V^{*} \otimes V$ of $\tilde{Q}$ in $X \times \tilde{Y}$.

Lemma C.5. The section $s_{\tilde{Q}}$ vanishes on the scheme $\Sigma$.

Consider the pullback of the exact sequence of lemma C.3 to $X \times \tilde{Y}$. Consider the cone $G^{\prime}$ of its first morphism twisted by $\mathcal{O}_{X}(3) \otimes \mathcal{O}_{\widetilde{Y}}(H)$, and the cone $G^{\prime \prime}$ of its third morphism twisted by $\mathcal{O}_{X}(1)$ as objects of $\mathcal{D}^{b}(X \times \tilde{Y})$, so that we have exact triangles

$$
\begin{gathered}
E_{1} \otimes \mathcal{O}_{\widetilde{Y}}(H-h) \stackrel{\tilde{e}^{\prime}}{\longrightarrow} E_{2} \otimes \Lambda^{2} \mathcal{U}_{\mathrm{Gr}(3, W)}^{\perp}(H) \longrightarrow G^{\prime} \\
E_{1} \otimes \mathcal{U}_{\mathrm{Gr}(3, W)}^{\perp} \stackrel{\tilde{e}^{\prime \prime}}{\longrightarrow} E_{2} \otimes \mathcal{O}_{\tilde{Y}} \longrightarrow G^{\prime \prime}
\end{gathered}
$$

Then the pullback of the exact sequence of lemma C.3 can be rewritten as exact triangle

$$
G^{\prime} \otimes\left(\mathcal{O}_{X}(-2) \otimes \mathcal{O}_{\tilde{Y}}(-H)\right)[1] \rightarrow G^{\prime \prime} \rightarrow \mathcal{O}_{\Sigma} \otimes \mathcal{O}_{X}(1) .
$$

It is clear that morphisms $\tilde{e}^{\prime}$ and $\tilde{e}^{\prime \prime}$ determine a structure of Q-representations on families $\left(\mathcal{O}_{\tilde{Y}}(H-\right.$ $\left.h), \Lambda^{2} \mathcal{U}_{\mathrm{Gr}(3, W)}^{\perp}(H)\right)$ and $\left(\mathcal{U}_{\mathrm{Gr}(3, W)}^{\perp}, \mathcal{O}_{\widetilde{Y}}\right)$ on $\widetilde{Y}$.

Lemma C.6. There exist a unique extension

$$
0 \rightarrow\left(\mathcal{O}_{\widetilde{Y}}(H-h), \Lambda^{2} \mathcal{U}_{\mathrm{Gr}(3, W)}^{\perp}(H)\right) \rightarrow\left(\tilde{F}_{1}, \tilde{F}_{2}\right) \rightarrow\left(\mathcal{U}_{\mathrm{Gr}(3, W)}^{\perp}, \mathcal{O}_{\widetilde{Y}}\right) \rightarrow 0
$$

in the category of families of Q-representations on $\tilde{Y}$ such that the cone of the induced morphism $\tilde{e}$ : $E_{1} \otimes \tilde{F}_{1} \rightarrow E_{2} \otimes \tilde{F}_{2}$ is a sheaf supported at $\tilde{Q}$. 
Lemma C.6 says that the family $\left(\tilde{F}_{1}, \tilde{F}_{2}\right)$ on $\tilde{Y}$ is a family of Q-representations and that on $X \times \tilde{Y}$ we have the following exact sequence:

$$
0 \rightarrow E_{1} \otimes \tilde{F}_{1} \stackrel{\tilde{e}}{\longrightarrow} E_{2} \otimes \tilde{F}_{2} \rightarrow \tilde{\imath}_{*} \tilde{\mathcal{E}} \rightarrow 0,
$$

where $\tilde{\mathcal{E}}$ is a coherent sheaf on $\tilde{Q}$.

Lemma C.7. The family $\left(\tilde{F}_{1}, \tilde{F}_{2}\right)$ induces a morphism $\phi: \tilde{Y} \rightarrow \bar{Y}_{4}$ such that $\tilde{g}=\bar{g}_{4} \circ \phi$.

Let $\tilde{Y}^{\circ}=\tilde{Y} \backslash \tilde{g}^{-1}\left(Z_{2}\right)$ and denote the restriction of $\tilde{g}$ and $\phi$ to $\tilde{Y}^{\circ}$ by the same symbols.

Recall that we have a sheaf of Azumaya algebras $\mathcal{A}_{4}$ on $Y_{4}$ and that $F_{2}$ is a locally projective $\mathcal{A}_{4}$-module on $Y_{4}$ of rank 4 . Let $\mathbb{P}_{Y_{4}, \mathcal{A}_{4}}\left(F_{2}^{*}\right)$ denote the moduli space of locally projective quotient $\mathcal{A}_{4}$-modules of rank 1. Then $\mathbb{P}_{Y_{4}, \mathcal{A}_{4}}\left(F_{2}^{*}\right)$ is a twisted $\mathbb{P}^{3}$-bundle over $Y_{4}$.

Proposition C.8. We have $\widetilde{Y}^{\circ}=\mathbb{P}_{Y_{4}, \mathcal{A}_{4}}\left(F_{2}^{*}\right)$.

Proof: The map $\tilde{Y}^{\circ} \rightarrow \mathbb{P}_{Y_{4}, \mathcal{A}_{4}}\left(F_{2}^{*}\right)$ can be constructed like in proposition A.9. Thus it remains to construct the inverse map. Choose a point $\tilde{y} \in \mathbb{P}_{Y_{4}, \mathcal{A}_{4}}\left(F_{2}^{*}\right)$. Let $\rho: W \otimes R_{1} \rightarrow R_{2}$ be the corresponding $(4,4)$-dimensional representation of the quiver $\mathrm{Q}$, and let $R_{2} \rightarrow \mathrm{k}$ be the corresponding one-dimensional quotient. Let $R_{2}^{\prime} \subset R_{2}$ be its kernel and consider the composition $E_{1} \otimes R_{1} \stackrel{e_{\rho}}{\longrightarrow} E_{2} \otimes R_{2} \rightarrow E_{2}$. Taking $\operatorname{Hom}\left(E_{1},-\right)$ we obtain a map $R_{1} \rightarrow W^{*}$. Let $R_{1}^{\prime}$ denote the kernel of this map. Then we have a diagram (54) and an exact sequence (55) Note that the sheaf $C$ is supported on the quadric $X_{y}=H_{g_{3}(y)} \cap X \subset X$ and its rank at the generic point of $X_{y}$ equals $\left(c_{1}\left(E_{2} \otimes R_{2}\right)-c_{1}\left(E_{1} \otimes R_{1}\right)\right) / 2=2$.

We claim that $\operatorname{dim} R^{\prime}=1$ and we are going to prove it by a case by case analysis.

Since $\left(R_{1}^{\prime}, R_{2}^{\prime}\right)$ is a subrepresentation of $\left(R_{1}, R_{2}\right)$ and $\left(R_{1}, R_{2}\right)$ is stable by lemma 5.5 we have

$$
\operatorname{dim} R_{1}^{\prime}<3,
$$

so we need to reject two cases: $\operatorname{dim} R_{1}^{\prime}=2$ and $\operatorname{dim} R_{1}^{\prime}=0$.

The case $\operatorname{dim} R_{1}^{\prime}=2$ is treated along the same lines as the case $\operatorname{dim} R_{1}^{\prime}=2$ in the proof of proposition A.9. The only difference is that in the case $\operatorname{dim} R_{1}^{\prime}=2$ the sheaf $T\left(C^{\prime}\right)$ can have rank 1 at the generic point of $X_{y}$, but it is easy to see that this is possible only in the case $C^{\prime} \cong \mathcal{O}_{X}(1) \oplus\left(\mathcal{O}_{X}(1) \oplus\right.$ $\left.\mathcal{O}_{X}(1)\right) /\left(\mathcal{O}_{X} \oplus \mathcal{O}_{X}\right)$, which means that $g_{4}(y) \in Z_{2}$.

Assume that $R_{1}^{\prime}=0$. Then the map $R_{1} \rightarrow W^{*}$ is injective, so we can consider $R_{1}$ as a 4 -dimensional subspace in $W^{*}$. If the cokernel of the morphism $K^{\prime \prime} \rightarrow C^{\prime}=\mathcal{O}_{X}(1)^{\oplus 3}$ is supported on a quadric $X_{y} \subset X$, then the morphism $\mathcal{O}_{X}(-1)^{\oplus 3} \cong \mathcal{O}_{X}(1)^{\oplus 3} \otimes \mathcal{O}_{X}(-2) \rightarrow \mathcal{O}_{X}(1)^{\oplus 3}$ factors through $K^{\prime \prime}$ and the cokernel of the corresponding morphism $\mathcal{O}_{X}(-1)^{\oplus 3} \rightarrow K^{\prime \prime}$ is supported on the same quadric $X_{y} \subset X$. On the other hand, it is easy to compute that $\operatorname{dim} \operatorname{Hom}\left(\mathcal{O}_{X}(-1), K^{\prime \prime}\right)=6$. Therefore the dimension of the set of such quadrics $X_{y} \subset X$ has dimension less than $\operatorname{dim} \operatorname{Gr}\left(4, W^{*}\right)+\operatorname{dim} \operatorname{Gr}(3,6)=8+9=17$. On the other hand, the set of such quadrics is evidently $\mathrm{GL}(W)$-invariant in $\mathbb{P}\left(V^{*}\right)=\mathbb{P}\left(S^{2} W^{*}\right)$, and it follows from the description of all $\mathrm{GL}(W)$-invariant closed subsets of $\mathbb{P}\left(S^{2} W^{*}\right)$ that this set is contained in $Z_{2}$.

Thus we have shown that if $g_{4}(y) \notin Z_{2}$ then $\operatorname{dim} R_{1}^{\prime}=1, \operatorname{dim} R_{1} / R_{1}^{\prime}=3$. The top and the bottom lines of the diagram (54) take form

$$
\begin{gathered}
0 \rightarrow \mathcal{O}_{X} \stackrel{e^{\prime}}{\rightarrow} \mathcal{O}_{X}(1)^{\oplus 3} \rightarrow C^{\prime} \rightarrow 0, \\
0 \rightarrow K^{\prime \prime} \rightarrow \mathcal{O}_{X}^{\oplus 3} \stackrel{e^{\prime \prime}}{\rightarrow} \mathcal{O}_{X}(1) \rightarrow C^{\prime \prime} \rightarrow 0 .
\end{gathered}
$$

The map $e^{\prime \prime}$ is given by a 3 -dimensional subspace of $W$, that is by a point $u \in \operatorname{Gr}(3, W)$. The same arguments as in the proof of proposition A.9 show that we can glue the sequence (68) twisted by $\mathcal{O}_{X}(-3)$ with the sequence (69) twisted by $\mathcal{O}_{X}(-1)$ into the following exact sequence

$$
0 \rightarrow \mathcal{O}_{X}(-3) \stackrel{e^{\prime}}{\longrightarrow} \mathcal{O}_{X}(-2)^{\oplus 3} \rightarrow \mathcal{O}_{X}(-1)^{\oplus 3} \stackrel{e^{\prime \prime}}{\rightarrow} \mathcal{O}_{X} \rightarrow C^{\prime \prime} \rightarrow 0 .
$$


Then it is easy to see that this sequence must coincide with the sequence of lemma C.3 restricted to a fiber of $X \times \operatorname{Gr}(3, W)$ over the point of $u \in \operatorname{Gr}(3, W)$. Associating to the point $\tilde{y}$ the point $u \in \operatorname{Gr}(3, W)$ we obtain a map $\mathbb{P}_{Y_{4}, \mathcal{A}_{4}}\left(F_{2}^{*}\right) \rightarrow \operatorname{Gr}(3, W)$. On the other hand, the support quadric $X_{y}$ of $C$ contains $\operatorname{supp}\left(C^{\prime \prime}\right)=\Sigma_{u}^{\prime}$, therefore the map

$$
\mathbb{P}_{Y_{4}, \mathcal{A}_{4}}\left(F_{2}^{*}\right) \rightarrow \operatorname{Gr}(3, W) \times \mathbb{P}\left(V^{*}\right), \quad \tilde{y} \mapsto\left(u, g_{4}(y)\right)
$$

factors through $\tilde{Y}=\mathbb{P}_{\operatorname{Gr}(3, W)}\left(p_{2 *}\left(J_{\Sigma^{\prime}} \otimes \mathcal{O}_{X}(2)\right)\right) \subset \operatorname{Gr}(3, W) \times \mathbb{P}\left(V^{*}\right)$. Moreover, it is clear that its image lies in $\tilde{Y}^{\circ}$, so we have constructed a map $\mathbb{P}_{Y_{4}, \mathcal{A}_{4}}\left(F_{2}^{*}\right) \rightarrow \widetilde{Y}^{\circ}$.

It remains to note that the constructed maps are mutually inverse.

Theorem C.9. The map $g_{4}: Y_{4} \rightarrow \mathbb{P}\left(V^{*}\right) \backslash Z_{2}$ is a double covering.

Proof: Since $\tilde{Y}^{\circ}$ is smooth it follows from proposition C.8 that $Y_{4}$ is smooth and moreover $\operatorname{dim} Y_{4}=$ $\operatorname{dim} \tilde{Y}^{\circ}-3=20$. On the other hand, since $\tilde{g}=\bar{g}_{4} \circ \phi, \tilde{g}$ and $\phi$ are proper and dominant, it follows that $g_{4}$ is a proper dominant map $Y_{4} \rightarrow \mathbb{P}\left(V^{*}\right) \backslash Z_{2}$. Since $Y_{4}$ and $\mathbb{P}\left(V^{*}\right) \backslash Z_{2}$ are smooth varieties of equal dimension and rankPic $Y_{4}=\operatorname{rank} \operatorname{Pic} \widetilde{Y}^{\circ}-1=1=\operatorname{rank} \operatorname{Pic}\left(\mathbb{P}\left(V^{*}\right) \backslash Z_{2}\right)$ it follows that $g_{4}$ is a finite covering. It remains to show that $\operatorname{deg} g_{4}=2$. This can be done by a direct calculation using the intersection theory of $\operatorname{Gr}(3, W)$. Alternatively, we can argue as follows.

Take a nondegenerate 2-form on $W^{*}$ and consider the zero locus of the corresponding section of the vector bundle $W / \mathcal{U}_{\mathrm{Gr}(3, W)}$ on $\operatorname{Gr}(3, W)$. It is clear that this is the Lagrangian Grassmannian $\operatorname{LGr}(3, W)$. Consider the fiber product $\tilde{Y}^{\circ} \times \operatorname{Gr}(3, W) \operatorname{LGr}(3, W)$. By lemma C.4 we have $\tilde{Y}^{\circ} \times{ }_{\operatorname{Gr}(3, W)} \operatorname{LGr}(3, W)$ is an open subset of $\mathbb{P}_{\operatorname{LGr}(3, W)}(\mathfrak{p})$, so and it is clear that the map $\tilde{g}_{\mid \mathbb{P}_{\operatorname{LGr}(3, W)}(\mathfrak{p})}: \mathbb{P}_{\operatorname{LGr}(3, W)}(\mathfrak{p}) \rightarrow \mathbb{P}\left(V^{*}\right)=\mathbb{P}(\mathfrak{g})$ is the projectivization of the partial Springer-Grothendieck map $\operatorname{Tot}(\mathfrak{p}) \rightarrow \mathfrak{g}$. Therefore, by $[\mathrm{Hu}]$ its degree equals $16 / 2=8$. On the other hand, since the restriction of $W / \mathcal{U}_{\operatorname{Gr}(3, W)}$ to the fiber $\mathbb{P}^{3}$ of $\widetilde{Y}^{\circ}$ over $Y_{4}$ is isomorphic to $\Omega_{\mathbb{P}^{3}}$ by $(67)$ we see that the degree of the map $\phi_{\mid \mathbb{P}_{\mathrm{LG}(3, W)}(\mathfrak{p})}: \mathbb{P}_{\mathrm{LGr}(3, W)}(\mathfrak{p}) \rightarrow Y_{4}$ equals $c_{3}\left(T_{\mathbb{P}^{3}}\right)=4$. Hence $\operatorname{deg} g_{4}=8 / 4=2$.

Corollary C.10. If the data (D.3)-(D.5) is given by $Y=Y_{4}, \mathcal{A}_{Y}=\mathcal{A}_{4}, \mathbf{Z}=\bar{Z}_{4}=Z_{2}, g=g_{4}$, with $\left(F_{1}, F_{2}\right)$ being the universal family, and with $\phi$ being induced by the Q-representation structure on $\left(F_{1}, F_{2}\right)$, then all conditions (C.1)-(C.8) are satisfied. Moreover, the branching locus of the map $g: Y \rightarrow \mathbb{P}\left(V^{*}\right) \backslash \mathbf{Z}$ is the hypersurface $X^{\vee} \backslash \mathbf{Z}$, where $X^{\vee} \subset \mathbb{P}\left(V^{*}\right)$ is the projectively dual sextic hypersurface.

Proof: Since the conditions (C.1)-(C.3), (45) and (51) are satisfied by lemma B.1, it follows from theorem 5.7 that the conditions (C.4)-(C.6) are true. On the other hand, (C.8) is true because $\mathbf{Z}$ is the locus of rank 4 quadrics by lemma C.2 and $\operatorname{dim} \mathbf{Z} \cap H=17-1=16$, while $N-i-2=21-3-2=16$. Finally, by theorem C.9 the map $Y=Y_{4} \rightarrow \mathbb{P}\left(V^{*}\right)$ is a double covering and $\operatorname{codim}_{Y \times Y}\left(Y \times_{\mathbb{P}\left(V^{*}\right)} Y\right)=\operatorname{dim} Y=$ $\operatorname{dim} \mathbb{P}\left(V^{*}\right)=20$, hence $\operatorname{dim} X+N-\operatorname{codim}_{Y \times Y}\left(Y \times_{\mathbb{P}\left(V^{*}\right)} Y\right)=5+21-20=6=2 i$, and (C.7) is true.

Finally, we note that the branching locus of $g$ is nothing but the singular locus of $g$, hence it coincides with $X^{\vee} \backslash \mathbf{Z}$ by corollary 4.30 .

\section{Appendix D. Azumaya algebraic Varieties}

In this section we work out some basic facts about Azumaya varieties. All algebraic varieties in this section are assumed to be embeddable.

Spaces, categories and functors.

Definition D.1. An Azumaya algebraic variety over a field $\mathrm{k}$ is a pair $\left(X, \mathcal{A}_{X}\right)$, where $X$ is an algebraic variety of finite type over $\mathrm{k}$ and $\mathcal{A}_{X}$ is a sheaf of semisimple $\mathcal{O}_{X}$-algebras which is locally free of finite rank over $\mathcal{O}_{X}$. A morphism of Azumaya varieties $f:\left(X, \mathcal{A}_{X}\right) \rightarrow\left(Y, \mathcal{A}_{Y}\right)$ is a pair $\left(f_{\circ}, f_{\mathcal{A}}\right)$, where $f_{\circ}: X \rightarrow Y$ 
is a morphism of algebraic varieties, and $f_{\mathcal{A}}: f_{\circ}^{*} \mathcal{A}_{Y} \rightarrow \mathcal{A}_{X}$ is a homomorphism of $f_{\circ}^{*} \mathcal{O}_{Y} \cong \mathcal{O}_{X}$-algebras. The composition of morphisms is defined naturally.

It is clear that Azumaya algebraic varieties over $\mathrm{k}$ form a category. Every algebraic variety $X$ can be considered as an Azumaya variety by taking $\mathcal{A}_{X}=\mathcal{O}_{X}$. Thus the category of algebraic varieties can be considered as a full subcategory of the category of Azumaya varieties. For each Azumaya variety $\left(X, \mathcal{A}_{X}\right)$ we have a canonical morphism $\pi_{X}:\left(X, \mathcal{A}_{X}\right) \rightarrow X$, the structure morphism of $\left(X, \mathcal{A}_{X}\right)$.

With each Azumaya algebraic variety $\left(X, \mathcal{A}_{X}\right)$ we associate abelian categories $\mathrm{Q} \operatorname{coh}\left(X, \mathcal{A}_{X}\right)$ and $\operatorname{Coh}\left(X, \mathcal{A}_{X}\right)$ of quasicoherent and coherent sheaves of right $\mathcal{A}_{X}$-modules on $X$ and their bounded and unbounded derived categories $\mathcal{D}_{q c}^{?}\left(X, \mathcal{A}_{X}\right)$ and $\mathcal{D}^{?}\left(X, \mathcal{A}_{X}\right)$, where $?=b,-,+$ or nothing.

Remark D.2. Recall that the Morita-equivalent algebras $\mathcal{A}_{X}$ and $\mathcal{A}_{X}^{\prime}$ give rise to equivalent categories $\operatorname{Coh}\left(X, \mathcal{A}_{X}\right)$ and $\operatorname{Coh}\left(X, \mathcal{A}_{X}^{\prime}\right)$. In particular, if $\mathcal{A}_{X} \cong \mathcal{E} n d(\mathcal{V})$, the algebra of endomorphisms of a vector bundle $\mathcal{V}$, then $\operatorname{Coh}(X) \cong \operatorname{Coh}\left(X, \mathcal{A}_{X}\right)$, the equivalence is given by tensoring with $\mathcal{V}$. However, we prefer to distinguish between Morita-equivalent Azumaya varieties.

Definition D.3. A morphism of Azumaya varieties $f:\left(X, \mathcal{A}_{X}\right) \rightarrow\left(Y, \mathcal{A}_{Y}\right)$ is called strict, if $\mathcal{A}_{X} \cong f_{0}^{*} \mathcal{A}_{Y}$ and $f_{\mathcal{A}}$ is the identity. Similarly, morphism $f$ is called an extension, if $X \cong Y$ and $f_{\circ}$ is the identity.

Every morphism $f:\left(X, \mathcal{A}_{X}\right) \rightarrow\left(Y, \mathcal{A}_{Y}\right)$ admits the following canonical decomposition:

$$
\left(X, \mathcal{A}_{X}\right) \stackrel{f^{e}}{\longrightarrow}\left(X, f_{\circ}^{*} \mathcal{A}_{Y}\right) \stackrel{f^{s}}{\longrightarrow}\left(Y, \mathcal{A}_{Y}\right),
$$

where $f^{e}$ is an extension, and $f^{s}$ is strict.

If $f:\left(X, \mathcal{A}_{X}\right) \rightarrow\left(Y, \mathcal{A}_{Y}\right)$ is a morphism of Azumaya varieties and $F \in \operatorname{Coh}\left(X, \mathcal{A}_{X}\right)$, we define $R^{0} f_{*} F \in \operatorname{Coh}\left(Y, \mathcal{A}_{Y}\right)$ as the sheaf $R^{0} f_{\text {o* }} F$ with an $\mathcal{A}_{Y}$-module structure induced by the homomorphism

$$
R^{0} f_{\circ *} F \otimes \mathcal{A}_{Y} \cong R^{0} f_{\circ *}\left(F \otimes f_{\circ}^{*} \mathcal{A}_{Y}\right) \stackrel{f_{\mathcal{A}}}{\longrightarrow} R^{0} f_{\circ *}\left(F \otimes \mathcal{A}_{X}\right) \rightarrow R^{0} f_{\circ *} F .
$$

It is clear that $R^{0} f_{*}$ is a functor and that exactness properties of $R^{0} f_{*}$ are the same as those of $R^{0} f_{\text {o* }_{*} \text {. If }}$ $f:\left(X, \mathcal{A}_{X}\right) \rightarrow$ Speck is the projection to a point, we denote the pushforward functor by $\Gamma(X,-)$. Note that the pushforward with respect to the structure morphism $\pi_{X}$ is just the forgetting of the $\mathcal{A}_{X}$-module structure.

Similarly, for $G \in \operatorname{Coh}\left(Y, \mathcal{A}_{Y}\right)$ we define $L_{0} f^{*} G \in \operatorname{Coh}\left(X, \mathcal{A}_{X}\right)$ as

$$
L_{0} f^{*} G=L_{0} f_{\circ}^{*} G \otimes_{f_{\circ}^{*} \mathcal{A}_{Y}} \mathcal{A}_{X} .
$$

Then $L_{0} f^{*}$ is also a functor. Note that if $f$ is a strict morphism then we have $L_{0} f^{*}=L_{0} f_{\circ}^{*}$.

If $F \in \operatorname{Coh}\left(X, \mathcal{A}_{X}\right)$ and $F^{\prime} \in \operatorname{Coh}\left(X, \mathcal{A}_{X}^{\text {opp }}\right)$, where $\mathcal{A}_{X}^{\text {opp }}$ is the opposite algebra, we can consider their tensor product over $\mathcal{A}_{X}, F \otimes_{\mathcal{A}_{X}} F^{\prime} \in \operatorname{Coh}(X)$. Note, that if $F$ (resp. $F^{\prime}$ ) admits some additional module structure, commuting with the structure of the right (resp. left) $\mathcal{A}_{X}$-module, then the tensor product $F \otimes_{\mathcal{A}_{X}} F^{\prime}$ preserves this structure. For example, if $F^{\prime}$ is a sheaf of $\mathcal{A}_{X}$-bimodules, then $F \otimes_{\mathcal{A}_{X}} F^{\prime} \in$ $\operatorname{Coh}\left(X, \mathcal{A}_{X}\right)$.

Similarly, for $F, F^{\prime} \in \operatorname{Coh}\left(X, \mathcal{A}_{X}\right)$ we can consider the sheaf $\mathcal{H}_{o} m_{\mathcal{A}_{X}}\left(F, F^{\prime}\right) \in \operatorname{Coh}(X)$ of local homomorphisms of $\mathcal{A}_{X}$-modules. Note, that if $F$ (resp. $F^{\prime}$ ) admits some additional module structure, commuting with the structure of the right $\mathcal{A}_{X}$-module, then the sheaf $\mathcal{H}_{o m_{\mathcal{A}_{X}}}\left(F, F^{\prime}\right)$ preserves this structure. For example, if $F^{\prime}$ is a sheaf of $\mathcal{A}_{X}$-bimodules, then $\mathcal{H}$ om $\mathcal{A}_{X}\left(F, F^{\prime}\right) \in \operatorname{Coh}\left(X, \mathcal{A}_{X}\right)$.

Lemma D.4. A sheaf $F \in \operatorname{Coh}\left(X, \mathcal{A}_{X}\right)$ is locally projective over $\mathcal{A}_{X}$ in the Zariski topology iff $\pi_{X *} F \in$ $\operatorname{Coh}(X)$ is locally free, where $\pi_{X}:\left(X, \mathcal{A}_{X}\right) \rightarrow X$ is the structure morphism.

Proof: Note that $\mathcal{A}_{X}^{\mathrm{opp}} \otimes \mathcal{A}_{X}$ is a sheaf of matrix algebras, therefore $\mathcal{A}_{X}$ considered as a $\mathcal{A}_{X}^{\mathrm{opp}} \otimes \mathcal{A}_{X}$-module is locally projective (in the Zariski topology), i.e. locally is a direct summand of $\mathcal{A}_{X}^{\mathrm{opp}} \otimes \mathcal{A}_{X}$. It follows that $F \cong F \otimes_{\mathcal{A}_{X}} \mathcal{A}_{X}$ is locally a direct summand of $F \otimes_{\mathcal{A}_{X}}\left(\mathcal{A}_{X}^{\mathrm{opp}} \otimes \mathcal{A}_{X}\right) \cong F \otimes_{\mathcal{O}_{X}} \mathcal{A}_{X}$, a free $\mathcal{A}_{X}$-module. 
It is clear that the category of quasicoherent $\mathcal{A}_{X}$-modules on an Azumaya variety $\left(X, \mathcal{A}_{X}\right)$ admits enough injectives and enough locally free objects. Therefore, the pushforward, the pullback, the tensor product and the $\mathcal{H}$ om functors can be extended to the derived categories in a natural way. From now on we use the notation $f_{*}, f^{*}, \otimes_{\mathcal{A}_{X}}$ and $\mathrm{RH} \mathcal{H}_{\mathcal{A}_{X}}$ to denote the derived functors. The derived functor of $\Gamma(X,-)$ is denoted by $\mathrm{R} \Gamma(X,-)$.

Remark D.5. Note that

$$
F \otimes_{\mathcal{A}_{X}} G \cong\left(F \otimes_{\mathcal{O}_{X}} G\right) \otimes_{\mathcal{A}_{X} \otimes \mathcal{A}_{X}^{\text {opp }}} \mathcal{A}_{X} \quad \text { and } \quad \operatorname{RH}_{\mathcal{H}} o m_{\mathcal{A}_{X}}(F, G) \cong \mathcal{H} o m_{\mathcal{A}_{X} \otimes \mathcal{A}_{X}^{\text {opp }}}\left(\mathcal{A}_{X}, \mathrm{R} \mathcal{H} o m_{\mathcal{O}_{X}}(F, G)\right) .
$$

Therefore the bar-resolution

$$
\cdots \rightarrow \mathcal{A}_{X} \otimes \mathcal{A}_{X}^{\otimes 2} \otimes \mathcal{A}_{X}^{\mathrm{opp}} \rightarrow \mathcal{A}_{X} \otimes \mathcal{A}_{X} \otimes \mathcal{A}_{X}^{\mathrm{opp}} \rightarrow \mathcal{A}_{X} \otimes \mathcal{A}_{X}^{\mathrm{opp}} \rightarrow \mathcal{A}_{X} \rightarrow 0
$$

gives the bar-resolutions of the functors $\otimes_{\mathcal{A}_{X}}$ and $\mathrm{R} \mathcal{H} o m_{\mathcal{A}_{X}}$

$$
\begin{aligned}
& \cdots \rightarrow F \otimes_{\mathcal{O}_{X}} \mathcal{A}_{X}^{\otimes 2} \otimes_{\mathcal{O}_{X}} G \rightarrow F \otimes_{\mathcal{O}_{X}} \mathcal{A}_{X} \otimes_{\mathcal{O}_{X}} G \rightarrow F \otimes_{\mathcal{O}_{X}} G \rightarrow F \otimes_{\mathcal{A}_{X}} G \rightarrow 0 \\
& 0 \rightarrow \operatorname{RH} \operatorname{Rom}_{\mathcal{A}_{X}}(F, G) \rightarrow \operatorname{RHom} \mathcal{O}_{X}(F, G) \rightarrow \operatorname{RHom}{\mathcal{O}_{X}}\left(F \otimes_{\mathcal{O}_{X}} \mathcal{A}_{X}, G\right) \rightarrow \ldots
\end{aligned}
$$

Remark D.6. If the algebra $\mathcal{A}_{X}$ is commutative then the category $\operatorname{Coh}\left(X, \mathcal{A}_{X}\right)$ is equivalent to the category of coherent sheaves on the algebraic variety $\operatorname{Spec}_{X} \mathcal{A}_{X}$ (the relative spectrum) which is étale over $X$. Following this analogy we consider a pair $\left(X, \mathcal{A}_{X}\right)$ in a noncommutative case as a noncommutative étale covering of $X$.

Finally, we define the twisted pull-back functor $f^{!}: \mathcal{D}^{+}\left(Y, \mathcal{A}_{Y}\right) \rightarrow \mathcal{D}^{+}\left(X, \mathcal{A}_{X}\right)$ extending the usual twisted pull-back functor defined in $[\mathrm{H}]$ to the category of Azumaya varieties.

The definition for a strict morphism is the same as in loc. cit.. If $f$ is strict and smooth we define

$$
f^{!}(G)=f^{*} G \otimes_{\mathcal{O}_{X}} \omega_{X / Y}[\operatorname{dim} X-\operatorname{dim} Y],
$$

and if $f_{\circ}$ is strict and finite we define

$$
f^{!} G=\mathrm{RH}^{\circ} m_{\mathcal{A}_{Y}}\left(f_{\circ *} \mathcal{A}_{X}, G\right),
$$

where we identify the category $\mathcal{D}^{+}\left(X, \mathcal{A}_{X}\right)$ with $\mathcal{D}^{+}\left(Y, f_{\circ *} \mathcal{A}_{X}\right)$ via the functor $f_{\text {o* }}$. Now assume that $f_{\circ}$ is embeddable (this is always the case when $X$ is embeddable). Decomposing $f$ into a composition $f_{\circ}=f_{\circ}^{\prime} \circ f_{\circ}^{\prime \prime}$ with $f_{\circ}^{\prime}$ smooth and $f_{\circ}^{\prime \prime}$ finite we define $f^{!}=f^{\prime \prime} ! \circ f^{\prime}$. A standard verification (loc. cit.) shows that this definition doesn't depend on a choice of a decomposition $f_{\circ}=f_{\circ}^{\prime} \circ f_{\circ}^{\prime \prime}$.

Now, for an arbitrary morphism $f:\left(X, \mathcal{A}_{X}\right) \rightarrow\left(Y, \mathcal{A}_{Y}\right)$ we consider its canonical decomposition $f=f^{s} \circ f^{e}$ with $f^{s}$ strict and $f^{e}$ extension, note that for any $G \in \mathcal{D}^{+}\left(Y, \mathcal{A}_{Y}\right)$ we have $f^{s !} G \in \mathcal{D}^{+}\left(X, f_{\circ}^{*} \mathcal{A}_{Y}\right)$ and define $f^{!} G$ by the formula

$$
f^{!} G=\mathrm{RH}^{\circ} m_{f_{\circ}^{*} \mathcal{A}_{Y}}\left(\mathcal{A}_{X}, f^{s !} G\right) .
$$

Lemma D.7. If $f$ is strict then $f^{!}=f_{0}^{!}$. Moreover

$$
\begin{array}{ll}
f_{\circ}^{!} F=f_{\circ}^{*} F \otimes_{\mathcal{O}_{X}} \operatorname{det} \mathcal{N}_{X / Y}[\operatorname{dim} X-\operatorname{dim} Y], & \text { if } f \text { is a locally complete intersection } \\
f_{\circ}^{!} F=f_{\circ}^{*} F \otimes_{\mathcal{O}_{X}} \omega_{X / Y}[\operatorname{dim} X-\operatorname{dim} Y], & \text { if } f_{\circ} \text { is smooth. }
\end{array}
$$

Proof: Evident.

\section{Relations between functors.}

Lemma D.8 (Functoriality of the pushforward). If $\left(X, \mathcal{A}_{X}\right) \stackrel{f}{\longrightarrow}\left(Y, \mathcal{A}_{Y}\right) \stackrel{g}{\longrightarrow}\left(Z, \mathcal{A}_{Z}\right)$ are morphisms of Azumaya varieties then $g_{*} f_{*} \cong(g f)_{*}$ on $\mathcal{D}\left(X, \mathcal{A}_{X}\right)$, a functorial isomorphism. In particular, $\mathrm{R} \Gamma\left(Y, f_{*}(-)\right) \cong \mathrm{R} \Gamma(X,-)$. 
Proof: Since the pushforward functor for Azumaya varieties coincides with the usual pushforward, the objects $R^{0} g_{*} R^{0} f_{*}(F)$ and $R^{0}(g f)_{*}(F)$ are identified in $\operatorname{Coh}(Z)$ by a natural isomorphism for any $F \in$ $\operatorname{Coh}\left(X, \mathcal{A}_{X}\right)$. Moreover, it is clear that this isomorphism identifies also the $\mathcal{A}_{Z}$-module structures on them. Therefore $R^{0} g_{*} R^{0} f_{*} \cong R^{0}(g f)_{*}$ as functors $\operatorname{Coh}\left(X, \mathcal{A}_{X}\right) \rightarrow \operatorname{Coh}\left(Z, \mathcal{A}_{Z}\right)$ whereof we deduce an isomorphism of derived functors as well.

Lemma D.9 (Functoriality of the pullback). If $\left(X, \mathcal{A}_{X}\right) \stackrel{f}{\longrightarrow}\left(Y, \mathcal{A}_{Y}\right) \stackrel{g}{\longrightarrow}\left(Z, \mathcal{A}_{Z}\right)$ are morphisms of Azumaya varieties then $f^{*} g^{*} \cong(g f)^{*}$ on $\mathcal{D}^{-}\left(Z, \mathcal{A}_{Z}\right)$. Moreover, if both $f$ and $g$ have finite Tor-dimension then $f^{*} g^{*} \cong(g f)^{*}$ on $\mathcal{D}\left(Z, \mathcal{A}_{Z}\right)$.

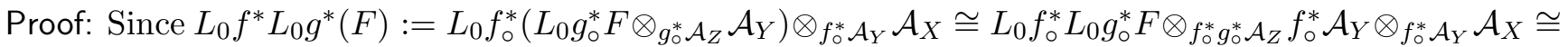

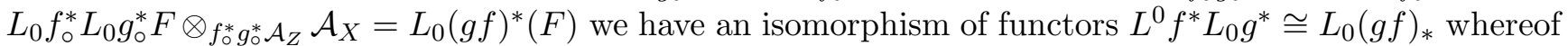
we deduce an isomorphism of derived functors as well.

Lemma D.10 (Associativity of the tensor product). If $F \in \mathcal{D}^{-}\left(X, \mathcal{A}_{1}\right), G \in \mathcal{D}^{-}\left(X, \mathcal{A}_{1}^{\text {opp }} \otimes \mathcal{A}_{2}\right)$, and $H \in \mathcal{D}^{-}\left(X, \mathcal{A}_{2}^{\text {opp }}\right)$ then $\left(F \otimes_{\mathcal{A}_{1}} G\right) \otimes_{\mathcal{A}_{2}} H \cong F \otimes_{\mathcal{A}_{1}}\left(G \otimes_{\mathcal{A}_{2}} H\right)$.

Proof: Similarly.

Lemma D.11 (Commutativity of the tensor product). If $F \in \mathcal{D}^{-}\left(X, \mathcal{A}_{X}\right)$ and $G \in \mathcal{D}^{-}\left(Y, \mathcal{A}_{X}^{\text {opp }}\right)$ then $F \otimes_{\mathcal{A}_{X}} G \cong G \otimes_{\mathcal{A}_{X}^{\text {opp }}} F$.

Proof: Similarly.

Lemma D.12 (The projection formula). If $f:\left(X, \mathcal{A}_{X}\right) \rightarrow\left(Y, \mathcal{A}_{Y}\right)$ is a morphism of Azumaya varieties, $F \in \mathcal{D}^{-}\left(X, \mathcal{A}_{X}\right), G \in \mathcal{D}^{-}\left(Y, \mathcal{A}_{Y}^{\text {opp }}\right)$, and $H \in \mathcal{D}^{-}\left(X, \mathcal{O}_{X}\right)$ then

$$
f_{*}\left(F \otimes_{\mathcal{A}_{X}} f^{*} G\right) \cong f_{*} F \otimes_{\mathcal{A}_{Y}} G, \quad \text { and } \quad f_{*}\left(f_{\circ}^{*} G \otimes_{\mathcal{O}_{X}} H\right) \cong G \otimes_{\mathcal{O}_{Y}} f_{*} H .
$$

Moreover, if $f$ is strict, $F \in \mathcal{D}^{-}\left(X, \mathcal{O}_{X}\right)$ and $G \in \mathcal{D}^{-}\left(Y, \mathcal{A}_{Y}\right)$ then $f_{*}\left(F \otimes_{\mathcal{O}_{X}} f^{*} G\right) \cong f_{*} F \otimes_{\mathcal{O}_{Y}} G$.

Proof: First of all assume that $f$ is strict. If $F$ is $f_{*}$-acyclic and $G$ is locally projective over $\mathcal{A}_{Y}$ then $f_{*}\left(F \otimes_{\mathcal{O}_{X}} f^{*} G\right) \cong f_{*} F \otimes_{\mathcal{O}_{Y}} G$ by the usual projection formula. Using the bar-resolution we then deduce that $f_{*}\left(F \otimes_{f_{\circ}^{*} \mathcal{A}_{Y}} f * G\right) \cong f_{*} F \otimes_{\mathcal{A}_{Y}} G$, the first isomorphism of the lemma. The standard argument [H], II.5.6 then proves this for all $F$ and $G$. On the other hand, if $f$ is an extension the first isomorphism of the lemma follows from $F \otimes_{\mathcal{A}_{X}}\left(\mathcal{A}_{X} \otimes_{\mathcal{A}_{Y}} G\right) \cong F \otimes_{\mathcal{A}_{Y}} G$. Finally, if $f$ is an arbitrary morphism we consider its canonical decomposition into the product of a strict morphism and an extension and deduce the first isomorphism of the lemma from the above remarks.

The second isomorphism is just the usual projection formula, and it remains to note that it is compatible with the $\mathcal{A}_{Y}$-module structures.

Lemma D.13. If $F \in \mathcal{D}^{-}\left(X, \mathcal{A}_{X}\right), G \in \mathcal{D}^{+}\left(X, \mathcal{A}_{X}\right)$ then we have a functorial isomorphism $\operatorname{RHom}_{\mathcal{A}_{X}}(F, G) \cong \mathrm{R} \Gamma\left(X, \mathrm{R} \mathcal{H} o m_{\mathcal{A}_{X}}(F, G)\right)$.

Proof: We have evidently $\operatorname{Hom}_{\mathcal{A}_{X}}(F, G) \cong \Gamma\left(X, \mathcal{H}\right.$ om $\left._{\mathcal{A}_{X}}(F, G)\right)$ for all sheaves $F, G \in \operatorname{Coh}\left(X, \mathcal{A}_{X}\right)$. The standard argument shows that we still have this identity for the derived functors.

Lemma D.14. If $f:\left(X, \mathcal{A}_{X}\right) \rightarrow\left(Y, \mathcal{A}_{Y}\right)$ is a strict morphism, such that $f_{\circ}$ has finite Tor-dimension and $F \in \mathcal{D}^{-}\left(Y, \mathcal{A}_{Y}\right), G \in \mathcal{D}^{+}\left(Y, \mathcal{A}_{Y}\right)$, then $f_{\circ}^{*} \operatorname{RH}_{\mathcal{H}} m_{\mathcal{A}_{Y}}(F, G) \cong \operatorname{RHom} \mathcal{A}_{X}\left(f^{*} F, f^{*} G\right)$.

Proof: For the strict morphism $f$ we have $f^{*}=f_{\circ}^{*}$, so combining the usual formula for the pullback of $\mathrm{RH}$ om with the bar-resolution of $\mathrm{RH} \mathrm{Hom}_{\mathcal{A}_{Y}}$ we deduce the lemma. 
Lemma D.15. If $f:\left(X, \mathcal{A}_{X}\right) \rightarrow\left(Y, \mathcal{A}_{Y}\right)$ is a strict morphism, $F \in \mathcal{D}^{-}\left(Y, \mathcal{A}_{Y}\right), G \in \mathcal{D}^{-}\left(Y, \mathcal{A}_{Y}^{\text {opp }}\right)$, then $f_{\circ}^{*}\left(F \otimes_{\mathcal{A}_{Y}} G\right) \cong f^{*} F \otimes_{\mathcal{A}_{X}} f^{*} G$.

Proof: As above.

Lemma D.16. If $f:\left(X, \mathcal{A}_{X}\right) \rightarrow\left(Y, \mathcal{A}_{Y}\right)$ is a morphism, $F \in \mathcal{D}^{-}\left(Y, \mathcal{A}_{Y}\right)$ and $G \in \mathcal{D}^{-}\left(Y, \mathcal{O}_{Y}\right)$, then $f^{*}\left(F \otimes \mathcal{O}_{Y} G\right) \cong f^{*} F \otimes \mathcal{O}_{X} f_{\circ}^{*} G$.

Proof: We have $f^{*}\left(F \otimes \mathcal{O}_{Y} G\right) \cong f_{\circ}^{*}\left(G \otimes \mathcal{O}_{Y} F\right) \otimes_{f_{\circ}^{*} \mathcal{A}_{Y}} \mathcal{A}_{X} \cong\left(f_{\circ}^{*} G \otimes_{\mathcal{O}_{X}} f_{\circ}^{*} F\right) \otimes_{f_{\circ}^{*} \mathcal{A}_{Y}} \mathcal{A}_{X} \cong$ $f_{\circ}^{*} G \otimes_{\mathcal{O}_{X}}\left(f_{\circ}^{*} F \otimes_{f_{\circ}^{*} \mathcal{A}_{Y}} \mathcal{A}_{X}\right) \cong f_{\circ}^{*} G \otimes_{\mathcal{O}_{X}} f^{*} F \cong f^{*} F \otimes_{\mathcal{O}_{X}} f_{\circ}^{*} G$.

Lemma D.17. If $f:\left(X, \mathcal{A}_{X}\right) \rightarrow\left(Y, \mathcal{A}_{Y}\right)$ is a morphism, $F \in \mathcal{D}^{-}\left(Y, \mathcal{A}_{Y}\right)$ and $G \in \mathcal{D}^{+}\left(X, \mathcal{A}_{X}\right)$, then $f_{*} \mathrm{RHom}\left(f^{*} F, G\right) \cong \mathrm{RH} \operatorname{om}\left(F, f_{*} G\right)$. In particular, the functor $f^{*}$ is left adjoint to $f_{*}$.

Proof: If $f$ is a strict morphism then $f_{*}=f_{\circ *}$ and $f_{*} \operatorname{RHom}_{\mathcal{O}_{X}}\left(f^{*} F, G\right) \cong \operatorname{RHom} \mathcal{O}_{Y}\left(F, f_{*} G\right)$ by the usual adjointness property. Using the bar-resolution we then deduce that $f_{*} \operatorname{RHom}_{f_{\circ}^{*} \mathcal{A}_{Y}}\left(f^{*} F, G\right) \cong$ $\mathrm{RHom}_{\mathcal{A}_{Y}}\left(F, f_{*} G\right)$. On the other hand, if $f$ is an extension the isomorphism of the lemma follows from $\mathrm{RH}^{\circ o m_{\mathcal{A}_{Y}}}\left(F, f_{*} G\right) \cong \mathrm{RHom}_{\mathcal{A}_{Y}}(F, G) \cong \mathrm{R} \mathcal{H} o m_{\mathcal{A}_{X}}\left(F \otimes_{\mathcal{A}_{Y}} \mathcal{A}_{X}, G\right)=\mathrm{R} \mathcal{H} o m_{\mathcal{A}_{X}}\left(f^{*} F, G\right)$. If $f$ is an arbitrary morphism we consider its canonical decomposition into the product of a strict morphism and an extension and deduce the first isomorphism of the lemma from the functoriality of the pullback and of the pushforward.

Flatness and smoothness.

Definition D.18. A morphism of Azumaya varieties $f:\left(X, \mathcal{A}_{X}\right) \rightarrow\left(Y, \mathcal{A}_{Y}\right)$ is called flat, if the pullback functor $f^{*}: \mathcal{D}\left(Y, \mathcal{A}_{Y}\right) \rightarrow \mathcal{D}\left(X, \mathcal{A}_{X}\right)$ is exact with respect to the standard t-structures.

Lemma D.19. Morphism $f:\left(X, \mathcal{A}_{X}\right) \rightarrow\left(Y, \mathcal{A}_{Y}\right)$ is flat iff $f_{\circ}: X \rightarrow Y$ is flat.

Proof: Let $\pi_{X}:\left(X, \mathcal{A}_{X}\right) \rightarrow X$, and $\pi_{Y}:\left(Y, \mathcal{A}_{Y}\right) \rightarrow Y$ be the structure morphisms. It is clear that $\pi_{X}^{*}$ and $\pi_{Y}^{*}$ are exact and conservative $\left(\pi_{X}^{*} F=0\right.$ implies $\left.F=0\right)$. On the other hand, we have $\pi_{Y} \circ f=f_{\circ} \circ \pi_{X}$, hence $f^{*} \pi_{Y}^{*}=\pi_{X}^{*} f_{\circ}^{*}$. If $f_{\circ}$ is not flat, then there exists $G \in \operatorname{Coh}(Y)$ such that $\mathcal{H}^{i}\left(f_{\circ}^{*} G\right) \neq 0$ for some $i<0$. Then $\mathcal{H}^{i}\left(f^{*} \pi_{Y}^{*} G\right)=\mathcal{H}^{i}\left(\pi_{X}^{*} f_{\circ}^{*} G\right)=\pi_{X}^{*} \mathcal{H}^{i}\left(f_{\circ}^{*} G\right) \neq 0$, while $\pi_{Y}^{*} G \in \operatorname{Coh}\left(Y, \mathcal{A}_{Y}\right)$. Hence $f^{*}$ is not exact. On the other hand, assume that $f_{\circ}$ is flat. Then the strict part $f^{s}$ of $f$ is flat. On the other hand, the extension part $f^{e}$ of $f$ is flat, because it coincides with the functor $F \mapsto F \otimes_{f_{\circ}^{*} \mathcal{A}_{Y}} \mathcal{A}_{X}$, and $\mathcal{A}_{X}$ is locally projective over $f_{\circ}^{*} \mathcal{A}_{Y}$ by lemma D.4.

Definition D.20. An object $F \in \mathcal{D}\left(X, \mathcal{A}_{X}\right)$ is called a perfect complex, if it is locally quasiisomorphic to a bounded complex of projective $\mathcal{A}_{X}$-modules of finite rank. We denote by $\mathcal{D}^{\text {perf }}\left(X, \mathcal{A}_{X}\right)$ the fully faithful subcategory of $\mathcal{D}\left(X, \mathcal{A}_{X}\right)$ formed by all perfect complexes.

Definition D.21. A triangulated category $\mathcal{D}$ is called Ext-bounded, if for any objects $F, F^{\prime} \in \mathcal{D}$ there exist $p, q \in \mathbb{Z}$ such that $\operatorname{Hom}_{\mathcal{D}}\left(F, F^{\prime}[t]\right)=0$ for $t \notin[p, q] \subset \mathbb{Z}$.

Lemma D.22. The following conditions for an Azumaya variety $\left(X, \mathcal{A}_{X}\right)$ are equivalent:

(i) the underlying algebraic variety $X$ is smooth;

(ii) $\mathcal{D}^{b}\left(X, \mathcal{A}_{X}\right)=\mathcal{D}^{\text {perf }}\left(X, \mathcal{A}_{X}\right)$.

(iii) the bounded derived category $\mathcal{D}^{b}\left(X, \mathcal{A}_{X}\right)$ is Ext-bounded.

Proof: $(i) \Longrightarrow(i i)$ : Take any $F \in \mathcal{D}^{b}\left(X, \mathcal{A}_{X}\right)$. Since $\operatorname{Coh}\left(X, \mathcal{A}_{X}\right)$ has enough locally free objects, we can construct a locally free over $\mathcal{A}_{X}$ resolution $F_{n} \rightarrow F_{n-1} \rightarrow \cdots \rightarrow F_{1} \rightarrow F_{0} \rightarrow F \rightarrow 0$ for arbitrary large $n$. On the other hand, if $n$ is sufficiently large, then $F_{n+1}:=\operatorname{Ker}\left(F_{n} \rightarrow F_{n-1}\right)$ is locally free over $\mathcal{O}_{X}$, 
hence locally projective over $\mathcal{A}_{X}$ by lemma D.4. Thus, $F$ is quasiisomorphic to a finite complex of locally projective $\mathcal{A}_{X}$-modules, so $F$ is a perfect complex.

$($ ii $) \Longrightarrow($ iii $)$ : Since $F$ is a perfect complex, we have $\operatorname{RHom}\left(F, F^{\prime}\right) \in \mathcal{D}^{[p, q]}(X)$ for some $p, q \in \mathbb{Z}$, hence $\operatorname{RHom}_{\mathcal{A}_{X}}\left(F, F^{\prime}\right)=\operatorname{R} \Gamma\left(X, \operatorname{RH}_{\text {or }}{ }_{\mathcal{A}_{X}}\left(F, F^{\prime}\right)\right) \in \mathcal{D}^{[p, q+\operatorname{dim} X]}(\mathcal{A} b)$.

$($ iii $) \Longrightarrow(i)$ : Let $\pi:\left(X, \mathcal{A}_{X}\right) \rightarrow X$ denote the structure morphism. Then

$$
\operatorname{RHom}_{\mathcal{A}_{X}}\left(\pi^{*} F, \pi^{*} F^{\prime}\right) \cong \operatorname{RHom}_{\mathcal{O}_{X}}\left(F, \pi_{*} \pi^{*} F^{\prime}\right) \cong \operatorname{RHom}_{\mathcal{O}_{X}}\left(F, F^{\prime} \otimes \mathcal{A}_{X}\right)
$$

for all $F, F^{\prime} \in \mathcal{D}^{b}\left(X, \mathcal{O}_{X}\right)$. If $X$ is not smooth, take $F=F^{\prime}$ to be the structure sheaf of a singular point. Then $F^{\prime} \otimes \mathcal{A}_{X} \cong F^{\prime \oplus \operatorname{rank} \mathcal{A}_{X}}$ and $\operatorname{Hom}_{\mathcal{O}_{X}}\left(F, F^{\prime} \otimes \mathcal{O}_{X} \mathcal{A}_{X}[t]\right) \neq 0$ for arbitrary large $t$. Hence $\mathcal{D}^{b}\left(X, \mathcal{A}_{X}\right)$ is not Ext-bounded.

Definition D.23. An Azumaya variety $\left(X, \mathcal{A}_{X}\right)$ is called smooth, if any of the equivalent conditions D.22 is satisfied. A morphism $f:\left(X, \mathcal{A}_{X}\right) \rightarrow\left(Y, \mathcal{A}_{Y}\right)$ of Azumaya varieties is called smooth if the underlying morphism $f_{0}: X \rightarrow Y$ is smooth.

Lemma D.24. If morphism $f:\left(X, \mathcal{A}_{X}\right) \rightarrow\left(Y, \mathcal{A}_{Y}\right)$ is smooth and $\left(Y, \mathcal{A}_{Y}\right)$ is a smooth Azumaya variety, then $\left(X, \mathcal{A}_{X}\right)$ is smooth. A composition of two smooth morphisms is smooth.

Proof: Evident.

\section{Further relations.}

Lemma D.25. If $F \in \mathcal{D}^{-}\left(X, \mathcal{A}_{1}\right), G \in \mathcal{D}^{\text {perf }}\left(X, \mathcal{A}_{2}\right)$ and $H \in \mathcal{D}^{+}\left(X, \mathcal{A}_{2}^{\text {opp }} \otimes \mathcal{A}_{1}\right)$ then we have $\mathrm{RH}^{\circ} m_{\mathcal{A}_{1}}\left(F, G \otimes_{\mathcal{A}_{2}} H\right) \cong G \otimes_{\mathcal{A}_{2}} \mathrm{RH} o_{\mathcal{A}_{1}}(F, H)$.

Proof: It is easy to construct a homomorphism from the RHS to the LHS. It is clear that it is an isomorphism for $G$ being locally projective, hence it is an isomorphism as well for $G$ being any perfect complex.

Lemma D.26. If either $F \in \mathcal{D}^{-}\left(X, \mathcal{A}_{X}\right), G \in \mathcal{D}^{\text {perf }}\left(X, \mathcal{A}_{X}\right)$ or $F \in \mathcal{D}^{\text {perf }}\left(X, \mathcal{A}_{X}\right), G \in \mathcal{D}^{+}\left(X, \mathcal{A}_{X}\right)$ then we have $\mathrm{RH} \operatorname{Hom}_{\mathcal{A}_{X}}(F, G) \cong G \otimes_{\mathcal{A}_{X}} \operatorname{RH}_{\mathcal{H}} o_{\mathcal{A}_{X}}\left(F, \mathcal{A}_{X}\right)$.

Proof: It is easy to construct a homomorphism from the RHS to the LHS. It is clear that it is an isomorphism when either $F$ or $G$ is locally projective. Hence it is an isomorphism when either $F$ or $G$ is perfect.

Lemma D.27. If $F \in \mathcal{D}^{-}\left(X, \mathcal{A}_{1}\right), G \in \mathcal{D}^{-}\left(X, \mathcal{A}_{1}^{\text {opp }} \otimes \mathcal{A}_{2}\right)$ and $H \in \mathcal{D}^{+}\left(X, \mathcal{A}_{2}\right)$ then we have $\mathrm{RH}^{\circ} m_{\mathcal{A}_{1}}\left(F, \mathrm{R} \mathcal{H} o m_{\mathcal{A}_{2}}(G, H)\right) \cong \mathrm{RH}^{\circ} m_{\mathcal{A}_{2}}\left(F \otimes \mathcal{A}_{1} G, H\right)$.

Proof: Evident.

For any $L \in \mathcal{D}^{-}\left(X, \mathcal{A}_{X}\right)$ we define the dual $L_{\mathcal{A}_{X}}^{*}:=\operatorname{R} \mathcal{H} o m_{\mathcal{A}_{X}}\left(L, \mathcal{A}_{X}\right) \in \mathcal{D}^{+}\left(X, \mathcal{A}_{X}^{\text {opp }}\right)$. It is clear that the dual of a bimodule is a bimodule, the dual of a perfect complex is a perfect complex, and $\left(L_{\mathcal{A}_{X}}^{*}\right)_{\mathcal{A}_{X}}^{*} \cong L$ (functorial isomorphism) for perfect complexes.

Lemma D.28. If $F \in \mathcal{D}^{-}\left(X, \mathcal{A}_{1}\right), G \in \mathcal{D}^{+}\left(X, \mathcal{A}_{2}\right)$, and $L \in \mathcal{D}^{\text {perf }}\left(X, \mathcal{A}_{2}^{\text {opp }} \otimes \mathcal{A}_{1}\right)$ then we have $\mathrm{RH}^{\circ} m_{\mathcal{A}_{1}}\left(F, G \otimes_{\mathcal{A}_{2}} L\right) \cong \mathrm{R} \mathcal{H}$ om $_{\mathcal{A}_{2}}\left(F \otimes_{\mathcal{A}_{1}} L_{\mathcal{A}_{2}}^{*}, G\right)$. Moreover, if $F \in \mathcal{D}^{-}\left(X, \mathcal{A}_{X}\right), G \in \mathcal{D}^{+}\left(X, \mathcal{A}_{X}\right)$, and $L \in \mathcal{D}^{\text {perf }}\left(X, \mathcal{O}_{X}\right)$ then we have $\mathrm{RH}_{\mathcal{H}} m_{\mathcal{A}_{X}}\left(F, G \otimes_{\mathcal{O}_{X}} L\right) \cong \mathrm{RHom}_{\mathcal{A}_{X}}\left(F \otimes_{\mathcal{O}_{X}} L_{\mathcal{O}_{X}}^{*}, G\right)$.

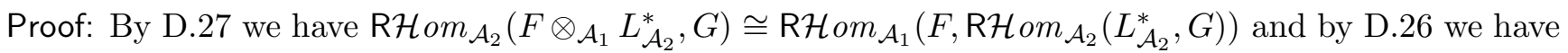
$\mathrm{RH}_{\mathrm{Hom}} \mathrm{A}_{\mathcal{A}_{2}}\left(L_{\mathcal{A}_{2}}^{*}, G\right) \cong G \otimes_{\mathcal{A}_{2}} \mathrm{RH}^{\mathrm{H} o m_{\mathcal{A}_{2}}}\left(L_{\mathcal{A}_{2}}^{*}, \mathcal{A}_{2}\right) \cong G \otimes_{\mathcal{A}_{2}} L$. The second claim can be proved similarly. 
Lemma D.29. If $f:\left(X, \mathcal{A}_{X}\right) \rightarrow\left(Y, \mathcal{A}_{Y}\right)$ is a morphism, $F \in \mathcal{D}^{-}\left(Y, \mathcal{A}_{Y}\right)$ and $G \in \mathcal{D}^{+}\left(Y, \mathcal{A}_{Y}\right)$ then we have $f_{\circ}^{!} \mathrm{R} \mathcal{H}$ om $_{\mathcal{A}_{Y}}(F, G) \cong \mathrm{R} \mathcal{H} o m_{f_{\circ}^{*} \mathcal{A}_{Y}}\left(f_{\circ}^{*} F, f_{\circ}^{!} G\right)$.

Proof: It is clear that $f_{0}^{!} \mathrm{R} \mathcal{H} \operatorname{om}_{\mathcal{O}_{Y}}(F, G) \cong \mathrm{R} \mathcal{H} \operatorname{om}_{\mathcal{O}_{X}}\left(f_{\circ}^{*} F, f_{\circ}^{!} G\right)$ by the usual properties of the twisted pullback. Using the bar-resolution we then deduce the claim.

Lemma D.30. If $f:\left(X, \mathcal{A}_{X}\right) \rightarrow\left(Y, \mathcal{A}_{Y}\right)$ is a morphism, $F \in \mathcal{D}^{\text {perf }}\left(Y, \mathcal{A}_{Y}\right)$ and $G \in \mathcal{D}^{+}\left(Y, \mathcal{A}_{Y}^{\text {opp }}\right)$ then we have $f_{\circ}^{!}\left(F \otimes_{\mathcal{A}_{Y}} G\right) \cong f_{\circ}^{*} F \otimes_{f_{\circ}^{*} \mathcal{A}_{Y}} f_{\circ}^{!} G$.

Proof: It is clear that $f_{\circ}^{!}\left(F \otimes_{\mathcal{O}_{Y}} G\right) \cong f_{\circ}^{*} F \otimes_{\mathcal{O}_{X}} f_{\circ}^{!} G$ by the usual properties of the twisted pullback. Using the bar-resolution we then deduce the claim.

Lemma D.31 (Functoriality of the twisted pullback). If $\left(X, \mathcal{A}_{X}\right) \stackrel{f}{\longrightarrow}\left(Y, \mathcal{A}_{Y}\right) \stackrel{g}{\longrightarrow}\left(Z, \mathcal{A}_{Z}\right)$ are morphisms of Azumaya varieties then $f^{!} g^{!} \cong(g f)^{!}$on $\mathcal{D}^{+}\left(Z, \mathcal{A}_{Z}\right)$.

Proof: First, consider the case when both $f$ and $g$ are extensions, so $X=Y=Z$. Then $f^{!} g^{!}(F) \cong$ $\mathrm{RH}^{\circ} m_{\mathcal{A}_{Y}}\left(\mathcal{A}_{X}, \mathrm{RH} \operatorname{Hom}_{\mathcal{A}_{Z}}\left(\mathcal{A}_{Y}, F\right)\right)$ which is isomorphic to $\mathrm{RH} o m_{\mathcal{A}_{Z}}\left(\mathcal{A}_{X}, F\right) \cong(g f)^{!}(F)$ by D.27.

Further, consider the case when both $f$ and $g$ are strict. Then $f^{!}=f_{0}^{!}, g^{!}=g_{\circ}^{!}$and the desired isomorphism follows from the functoriality of the twisted pullback functor for morphisms of algebraic varieties.

Since $f^{!} g^{!} \cong(g f)^{!}$if $f$ is an extension and $g$ is strict, it remains to show that $f^{!} g^{!} \cong(g f)^{!}$when $f$ is strict and $g$ is an extension. Indeed, in this case $Z=Y, \mathcal{A}_{X}=f_{\circ}^{*} \mathcal{A}_{Y}$, the canonical decomposition of $g f:\left(X, f_{\circ}^{*} \mathcal{A}_{Y}\right) \rightarrow\left(Y, \mathcal{A}_{Z}\right)$ takes form $\left(X, f_{\circ}^{*} \mathcal{A}_{Y}\right) \stackrel{f_{\circ}^{*} g_{\mathcal{A}}}{\longrightarrow}\left(X, f_{\circ}^{*} \mathcal{A}_{Z}\right) \stackrel{f_{\circ}}{\longrightarrow}\left(Y, \mathcal{A}_{Z}\right)$, we have $f^{!} g^{!}(G)=f_{\circ}^{!} \operatorname{RH}^{\prime} m_{\mathcal{A}_{Z}}\left(\mathcal{A}_{Y}, G\right),(g f)^{!}(G)=\mathrm{RH}_{o} m_{f_{\circ}^{*} \mathcal{A}_{Z}}\left(f_{\circ}^{*} \mathcal{A}_{Y}, f_{\circ}^{!} G\right)$ for all $G \in \mathcal{D}^{+}\left(Z, \mathcal{A}_{Z}\right)$ and it remains to apply D.29 with $F=\mathcal{A}_{Y}$.

Lemma D.32. If $f:\left(X, \mathcal{A}_{X}\right) \rightarrow\left(Y, \mathcal{A}_{Y}\right)$ is a morphism of Azumaya varieties $F \in \mathcal{D}^{+}\left(Y, \mathcal{A}_{Y}\right)$, and $G \in \mathcal{D}^{\text {perf }}\left(Y, \mathcal{O}_{Y}\right)$ then we have $f^{!}\left(F \otimes_{\mathcal{O}_{Y}} G\right) \cong f^{!} F \otimes_{\mathcal{O}_{X}} f_{\circ}^{*} G$.

Proof: By functoriality of the pullback and of the twisted pullback it suffices to prove this only for a strict morphism and for an extension. For a strict morphism $f$ we have $f^{!}=f_{0}^{!}$, hence we can use properties of the usual twisted pullback. Now assume that $f$ is an extension. Then by D.25 we have $f^{!}\left(F \otimes_{\mathcal{O}_{Y}} G\right) \cong \mathrm{RH}^{\circ o m_{\mathcal{A}_{Y}}}\left(\mathcal{A}_{X}, F \otimes_{\mathcal{O}_{Y}} G\right) \cong \mathrm{RH} \operatorname{om}_{\mathcal{A}_{Y}}\left(\mathcal{A}_{X}, F\right) \otimes_{\mathcal{O}_{Y}} G \cong f^{!} F \otimes_{\mathcal{O}_{X}} f_{\circ}^{*} G$, and we are done.

Lemma D.33. If $f:\left(X, \mathcal{A}_{X}\right) \rightarrow\left(Y, \mathcal{A}_{Y}\right)$ is a morphism of Azumaya varieties, $F \in \mathcal{D}^{+}\left(Y, \mathcal{A}_{Y}\right)$, and $G \in \mathcal{D}^{\text {perf }}\left(Y, \mathcal{O}_{Y}\right)$ then we have $f^{!} \mathrm{R \mathcal {H }} \operatorname{om}_{\mathcal{O}_{Y}}(F, G) \cong \mathrm{RH} \operatorname{Hom}_{\mathcal{O}_{X}}\left(f^{*} F, f_{\circ}^{!} G\right)$.

Proof: By functoriality of the pullback and of the twisted pullback it suffices to prove this only for a strict morphism and for an extension. For a strict morphism $f$ we have $f^{*}=f_{\circ}^{*}$ and $f^{!}=f_{\circ}^{!}$, hence we can use properties of the usual twisted pullback. Now assume that $f$ is an extension. Then we have

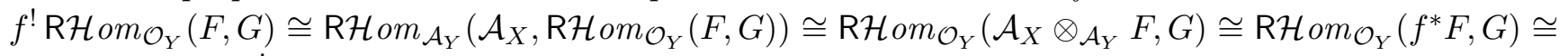
$\mathrm{RH} \operatorname{Rom}_{\mathcal{O}_{X}}\left(f^{*} F, f_{\circ}^{!} G\right)$, since $X=Y$ and $f_{\circ}=$ id.

Lemma D.34 (Duality). If $f:\left(X, \mathcal{A}_{X}\right) \rightarrow\left(Y, \mathcal{A}_{Y}\right)$ is a projective morphism of Azumaya varieties, $F \in \mathcal{D}^{-}\left(X, \mathcal{A}_{X}\right), G \in \mathcal{D}^{+}\left(Y, \mathcal{A}_{Y}\right)$ then we have $\mathrm{RH}_{\mathcal{H}} m_{\mathcal{A}_{Y}}\left(f_{*} F, G\right) \cong f_{*} \mathrm{RHom}_{\mathcal{A}_{X}}\left(F, f^{!} G\right)$.

Proof: By functoriality of the pushforward and of the twisted pullback it suffices to prove the duality only for a strict morphism and for an extension. For a strict morphism $f$ we have $f_{*}=f_{\circ *}, f^{!}=f_{\circ}^{!}$, and $\mathrm{RH}^{\circ} \operatorname{om}_{\mathcal{O}_{Y}}\left(f_{*} F, G\right) \cong f_{*} \mathrm{RH} \operatorname{om}_{\mathcal{O}_{X}}\left(F, f^{!} G\right)$ by the usual duality theorem. Using the bar-resolution we then 
deduce that $\mathrm{RH} \operatorname{Hom}_{\mathcal{A}_{Y}}\left(f_{*} F, G\right) \cong f_{*} \mathrm{RH} \operatorname{mom}_{\mathcal{A}_{X}}\left(F, f^{!} G\right)$. Now assume that $f$ is an extension. Then we have $f_{*} \operatorname{RH}_{\mathcal{H}} m_{\mathcal{A}_{X}}\left(F, f^{!} G\right) \cong \mathrm{R} \mathcal{H} o m_{\mathcal{A}_{X}}\left(F, \operatorname{RH}_{\mathcal{H}} m_{\mathcal{A}_{Y}}\left(\mathcal{A}_{X}, G\right)\right) \cong \mathrm{R} \mathcal{H} o m_{\mathcal{A}_{Y}}\left(F \otimes_{\mathcal{A}_{X}} \mathcal{A}_{X}, G\right) \cong \mathrm{R} \mathcal{H} o m_{\mathcal{A}_{Y}}(F, G) \cong$ $\mathrm{RHom}_{\mathcal{A}_{Y}}\left(f_{*} F, G\right)$ and we are done.

Corollary D.35. If $f:\left(X, \mathcal{A}_{X}\right) \rightarrow\left(Y, \mathcal{A}_{Y}\right)$ is a projective morphism of Azumaya varieties then the functor $f^{!}$is right adjoint to $f_{*}$ on the bounded derived category.

\section{Fiber products and base changes.}

Let $\left(X, \mathcal{A}_{X}\right)$ and $\left(Y, \mathcal{A}_{Y}\right)$ be Azumaya varieties. Then $\left(X \times Y, \mathcal{A}_{X} \otimes \mathcal{A}_{Y}\right)$ is also an Azumaya variety and the projections of $X \times Y$ to $X$ and $Y$ with embeddings of $\mathcal{A}_{X} \otimes \mathcal{O}_{Y}$ and $\mathcal{O}_{X} \otimes \mathcal{A}_{Y}$ to $\mathcal{A}_{X} \otimes \mathcal{A}_{Y}$ define maps from $\left(X \times Y, \mathcal{A}_{X} \otimes \mathcal{A}_{Y}\right)$ to $\left(X, \mathcal{A}_{X}\right)$ and $\left(Y, \mathcal{A}_{Y}\right)$. Note that $\left(X \times Y, \mathcal{A}_{X} \otimes \mathcal{A}_{Y}\right)$ is not a categorical product of $\left(X, \mathcal{A}_{X}\right)$ and $\left(Y, \mathcal{A}_{Y}\right)$. Nevertheless, we will denote it by $\left(X, \mathcal{A}_{X}\right) \times\left(Y, \mathcal{A}_{Y}\right)$.

Lemma D.36. If $p:\left(X, \mathcal{A}_{X}\right) \times\left(Y, \mathcal{A}_{Y}\right) \rightarrow\left(X, \mathcal{A}_{X}\right)$ is the projection then we have $p^{*} F \cong F \otimes \mathcal{A}_{Y}$ and $p^{!} F \cong F \otimes \mathrm{RH} \operatorname{Hom}_{\mathcal{O}_{Y}}\left(\mathcal{A}_{Y}, \omega_{Y}\right)[\operatorname{dim} Y]$.

Proof: Evident.

It is easy to see that in general the fiber products don't exist in the category of Azumaya varieties. However, there are important cases when they do exist.

Assume that $f:\left(X, \mathcal{A}_{X}\right) \rightarrow\left(S, \mathcal{A}_{S}\right)$ and $g:\left(Y, \mathcal{A}_{Y}\right) \rightarrow\left(S, \mathcal{A}_{S}\right)$ are morphisms of Azumaya varieties. Let $p_{\circ}: X \times_{S} Y \rightarrow X$ and $q_{\circ}: X \times_{S} Y \rightarrow Y$ denote the projections.

Lemma D.37. If $f$ is strict then $\left(X \times_{S} Y, q_{\circ}^{*} \mathcal{A}_{Y}\right)$ is a fiber product of $\left(X, \mathcal{A}_{X}\right)$ and $\left(Y, \mathcal{A}_{Y}\right)$ over $\left(S, \mathcal{A}_{S}\right)$. If $g$ is strict then $\left(X \times_{S} Y, p_{\circ}^{*} \mathcal{A}_{X}\right)$ is a fiber product of $\left(X, \mathcal{A}_{X}\right)$ and $\left(Y, \mathcal{A}_{Y}\right)$ over $\left(S, \mathcal{A}_{S}\right)$.

Proof: If $\phi:\left(Z, \mathcal{A}_{Z}\right) \rightarrow\left(X, \mathcal{A}_{X}\right)$ and $\psi:\left(Z, \mathcal{A}_{Z}\right) \rightarrow\left(Y, \mathcal{A}_{Y}\right)$ are morphisms of Azumaya varieties such that $f \circ \phi=g \circ \psi$ then $f_{\circ} \circ \phi_{\circ}=g_{\circ} \circ \psi_{\circ}$, hence there exists a morphism $\xi_{\circ}: Z \rightarrow X \times{ }_{S} Y$ such that $p_{\circ} \circ \xi_{\circ}=\phi_{\circ}$ and $q_{\circ} \circ \xi_{\circ}=\psi_{\circ}$. Moreover, if $f$ is strict then the map $\xi_{\circ}^{*} q_{\circ}^{*} \mathcal{A}_{Y} \cong \psi_{\circ}^{*} \mathcal{A}_{Y} \stackrel{\psi_{\mathcal{A}}}{\longrightarrow} \mathcal{A}_{Z}$ induces a morphism of Azumaya varieties $\left(Z, \mathcal{A}_{Z}\right) \rightarrow\left(X \times_{S} Y, q_{\circ}^{*} \mathcal{A}_{Y}\right)$. Similarly, if $g$ is strict then the map $\xi_{\circ}^{*} p_{\circ}^{*} \mathcal{A}_{X} \cong \phi_{\circ}^{*} \mathcal{A}_{X} \stackrel{\phi_{\mathcal{A}}}{\longrightarrow} \mathcal{A}_{Z}$ induces a morphism of Azumaya varieties $\left(Z, \mathcal{A}_{Z}\right) \rightarrow\left(X \times_{S} Y, p_{\circ}^{*} \mathcal{A}_{X}\right)$.

We often consider strict morphism $f:\left(X, \mathcal{A}_{X}\right) \rightarrow\left(Y, \mathcal{A}_{Y}\right)$ as a base change.

Lemma D.38. A base change preserves flatness, smoothness, projectivity, e.t.c. of a morphism.

Proof: Evident.

If the algebra $\mathcal{A}_{X}$ is noncommutative, the multiplication $\mathcal{A}_{X} \otimes \mathcal{A}_{X} \rightarrow \mathcal{A}_{X}$ is not a homomorphism of algebras. Therefore, there is no "diagonal embedding" in the category of Azumaya varieties. However, $\mathcal{A}_{X}$ is a $\mathcal{A}_{X} \otimes \mathcal{A}_{X}^{\text {opp }}$-module, thus "the structure sheaf of the diagonal", $\Delta_{*} \mathcal{A}_{X}$ on $X \times X$ can be considered as an object of $\operatorname{Coh}\left(X \times X, \mathcal{A}_{X} \otimes \mathcal{A}_{X}^{\text {opp }}\right)$.

Tor and Ext-amplitude.

Let $f:\left(X, \mathcal{A}_{X}\right) \rightarrow\left(Y, \mathcal{A}_{Y}\right)$ be a morphism of Azumaya varieties.

Definition D.39. An object $F \in \mathcal{D}\left(X, \mathcal{A}_{X}\right)$ has finite Tor-amplitude over $\left(Y, \mathcal{A}_{Y}\right)$, if there exist integers $p, q$ such that for any object $G \in \mathcal{D}^{[s, t]}\left(Y, \mathcal{A}_{Y}\right)$ we have $F \otimes_{\mathcal{A}_{X}} f^{*} G \in \mathcal{D}^{[p+s, q+t]}\left(X, \mathcal{O}_{X}\right)$. Morphism $f$ has finite Tor-dimension, if the sheaf $\mathcal{A}_{X}$ has finite Tor-amplitude over $\left(Y, \mathcal{A}_{Y}\right)$. Similarly, an object $F \in \mathcal{D}\left(X, \mathcal{A}_{X}\right)$ has finite Ext-amplitude over $\left(Y, \mathcal{A}_{Y}\right)$, if there exist integers $p, q$ such that for any object $G \in \mathcal{D}^{[s, t]}\left(Y, \mathcal{A}_{Y}\right)$ we have $\mathrm{RH} \operatorname{om}_{\mathcal{A}_{X}}\left(F, f^{!} G\right) \in \mathcal{D}^{[p+s, q+t]}\left(X, \mathcal{O}_{X}\right)$. Morphism $f$ has finite Ext-dimension, if the sheaf $\mathcal{A}_{X}$ has finite Ext-amplitude over $\left(Y, \mathcal{A}_{Y}\right)$. 
The full subcategory of $\mathcal{D}\left(X, \mathcal{A}_{X}\right)$ consisting of objects of finite Tor-amplitude over $\left(Y, \mathcal{A}_{Y}\right)$ is denoted by $\mathcal{D}_{f T d /\left(Y, \mathcal{A}_{Y}\right)}\left(X, \mathcal{A}_{X}\right)$. The full subcategory of $\mathcal{D}\left(X, \mathcal{A}_{X}\right)$ consisting of objects of finite Ext-amplitude over $\left(Y, \mathcal{A}_{Y}\right)$ is denoted by $\mathcal{D}_{f E d /\left(Y, \mathcal{A}_{Y}\right)}\left(X, \mathcal{A}_{X}\right)$. Both are triangulated subcategories of $\mathcal{D}^{b}\left(X, \mathcal{A}_{X}\right)$.

Lemma D.40. If $i:\left(X, \mathcal{A}_{X}\right) \rightarrow\left(X^{\prime}, \mathcal{A}_{X^{\prime}}\right)$ is a finite morphism of Azumaya varieties over $\left(Y, \mathcal{A}_{Y}\right)$ then $F \in \mathcal{D}_{f T d /\left(Y, \mathcal{A}_{Y}\right)}\left(X, \mathcal{A}_{X}\right) \Leftrightarrow i_{*} F \in \mathcal{D}_{f T d /\left(Y, \mathcal{A}_{Y}\right)}\left(X^{\prime}, \mathcal{A}_{X^{\prime}}\right)$ and $F \in \mathcal{D}_{f E d /\left(Y, \mathcal{A}_{Y}\right)}\left(X, \mathcal{A}_{X}\right) \Leftrightarrow i_{*} F \in$ $\mathcal{D}_{f E d /\left(Y, \mathcal{A}_{Y}\right)}\left(X^{\prime}, \mathcal{A}_{X^{\prime}}\right)$.

Proof: Use exactness of $i_{*}$, the isomorphisms $i_{*} F \otimes_{\mathcal{A}_{X^{\prime}}} f^{\prime *} G \cong i_{*}\left(F \otimes_{\mathcal{A}_{X}} i^{*} f^{\prime *} G\right) \cong i_{*}\left(F \otimes_{\mathcal{A}_{X}} f^{*} G\right)$ and $\mathrm{RH} \operatorname{Hom}_{\mathcal{A}_{X^{\prime}}}\left(i_{*} F, f^{\prime !} G\right) \cong i_{*} \mathrm{R} \mathcal{H} o m_{\mathcal{A}_{X}}\left(F, i^{!} f^{\prime !} G\right) \cong i_{*} \mathrm{RH}^{\circ} m_{\mathcal{A}_{X}}\left(F, f^{!} G\right)$.

Lemma D.41. If morphism $f:\left(X, \mathcal{A}_{X}\right) \rightarrow\left(Y, \mathcal{A}_{Y}\right)$ has finite Tor-dimension then any perfect complex on $\left(X, \mathcal{A}_{X}\right)$ has finite Tor-amplitude over $\left(Y, \mathcal{A}_{Y}\right)$. If morphism $f:\left(X, \mathcal{A}_{X}\right) \rightarrow\left(Y, \mathcal{A}_{Y}\right)$ has finite Extdimension then any perfect complex on $\left(X, \mathcal{A}_{X}\right)$ has finite Ext-amplitude over $\left(Y, \mathcal{A}_{Y}\right)$.

Proof: Evident.

Now we are going to prove the inverse statements for a smooth morphism. For this we will need the following fact about perfect complexes on usual algebraic varieties. This fact certainly must be well known, however I don't know a reference, so the proof is included here for completeness.

Lemma D.42. Assume that $f: X \rightarrow S$ is a smooth morphism of algebraic varieties and $F$ is a coherent sheaf on $X$. If $F$ is either flat or locally projective over $S$ then $F$ is a perfect complex.

Proof: The claim is local in $X$, so we can assume that $X$ is affine and the ideal of the diagonal $X \subset X \times{ }_{S} X$ is generated by a regular sequence $s=\left(s_{1}, \ldots, s_{n}\right)$ of functions on $X$. Then $\Delta_{*} \mathcal{O}_{X} \cong \operatorname{Kosz}_{X \times{ }_{S} X}(s) \cong$ $\Lambda^{\bullet}\left(\mathcal{O}_{X \times S}^{\oplus n}\right)$. Consider the diagram

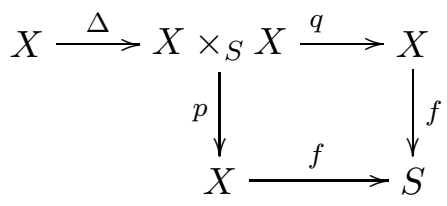

and note that $q \circ \Delta=p \circ \Delta=\mathrm{id}_{X}$, hence for any closed point $x \in X$ we have

$$
\begin{aligned}
& \mathcal{O}_{x} \cong q_{*} \Delta_{*} \Delta^{*} p^{*} \mathcal{O}_{x} \cong q_{*}\left(\Delta_{*} \mathcal{O}_{X} \otimes p^{*} \mathcal{O}_{x}\right) \cong q_{*}\left(\operatorname{Kosz}_{X \times_{S} X}(s) \otimes p^{*} \mathcal{O}_{x}\right), \\
& \mathcal{O}_{x} \cong q_{*} \Delta_{*} \Delta ! p^{!} \mathcal{O}_{x} \cong q_{*} \operatorname{RH} \operatorname{Hom}\left(\Delta_{*} \mathcal{O}_{X}, p^{!} \mathcal{O}_{x}\right) \cong q_{*} \operatorname{RHom}\left(\operatorname{Kosz}_{X \times_{S} X}(s), p^{!} \mathcal{O}_{x}\right) .
\end{aligned}
$$

But the flat base change implies that

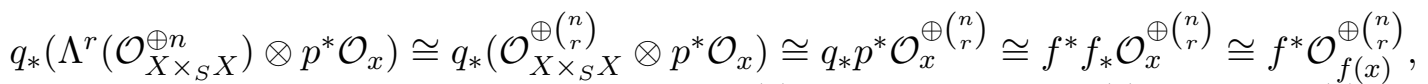

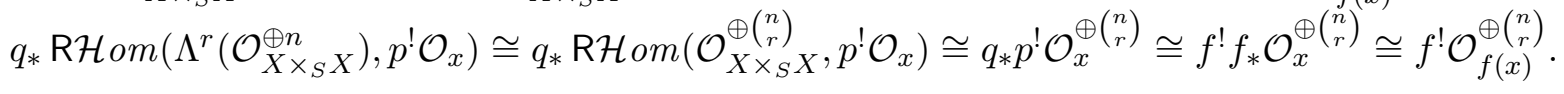

Thus every sheaf $\mathcal{O}_{x}$ on $X$ admits a length $n$ resolution either by sheaves, which are pullbacks of coherent sheaves on $S$, or by twisted pullbacks of coherent sheaves on $S$. It follows that $\operatorname{Tor}_{>n}\left(F, \mathcal{O}_{x}\right)=0$ for all $x \in X$ if $F$ is flat over $S$, and $\operatorname{Ext}^{>n}\left(F, \mathcal{O}_{x}\right)=0$ for all $x \in X$ if $F$ is locally projective over $S$. On the other hand, it is easy to construct a resolution of $F$ on $X$ of the form $0 \rightarrow F^{\prime} \rightarrow F_{n-1} \rightarrow \cdots \rightarrow F_{1} \rightarrow$ $F_{0} \rightarrow F \rightarrow 0$ with locally free $F_{i}$. Then it follows that $\operatorname{Tor}_{>0}\left(F^{\prime}, \mathcal{O}_{x}\right)=0$ for all $x \in X$ if $F$ is flat over $S$, and $\operatorname{Ext}^{>0}\left(F^{\prime}, \mathcal{O}_{x}\right)=0$ for all $x \in X$ if $F$ is locally projective over $S$. Hence in both cases $F^{\prime}$ is locally free, so $F$ is a perfect complex.

Lemma D.43. If $f:\left(X, \mathcal{A}_{X}\right) \rightarrow\left(Y, \mathcal{A}_{Y}\right)$ is a smooth morphism of Azumaya varieties then we have $\mathcal{D}_{f T d /\left(Y, \mathcal{A}_{Y}\right)}\left(X, \mathcal{A}_{X}\right)=\mathcal{D}^{\text {perf }}\left(X, \mathcal{A}_{X}\right)=\mathcal{D}_{f E d /\left(Y, \mathcal{A}_{Y}\right)}\left(X, \mathcal{A}_{X}\right)$. 
Proof: Any perfect complex has finite Tor-amplitude by lemma D.41. Take $F \in \mathcal{D}_{f T d /\left(Y, \mathcal{A}_{Y}\right)}\left(X, \mathcal{A}_{X}\right)$. Since $\operatorname{Coh}\left(X, \mathcal{A}_{X}\right)$ has enough locally free objects, we can construct a locally free over $\mathcal{A}_{X}$ resolution $F_{n-1} \rightarrow \cdots \rightarrow F_{1} \rightarrow F_{0} \rightarrow F \rightarrow 0$ for arbitrary large $n$. On the other hand, if $n$ is sufficiently large, then $F_{n}:=\operatorname{Ker}\left(F_{n-1} \rightarrow F_{n-2}\right)$ is flat over $\left(Y, \mathcal{A}_{Y}\right)$, hence $F_{n}$ is flat over $Y$ (since the canonical projection $\left(Y, \mathcal{A}_{Y}\right) \rightarrow Y$ is flat), hence $F_{n}$ is locally free over $\mathcal{O}_{X}$, hence $F_{n+1}$ is locally projective over $\mathcal{A}_{X}$ by lemma D.4. Therefore, any $F$ is quasiisomorphic to a finite complex of locally projective $\mathcal{A}_{X}$-modules, hence $F$ is a perfect complex. The same arguments show $\mathcal{D}_{f E d /\left(Y, \mathcal{A}_{Y}\right)}\left(X, \mathcal{A}_{X}\right)=\mathcal{D}^{\text {perf }}\left(X, \mathcal{A}_{X}\right)$.

Corollary D.44. $\mathcal{D}_{f T d /\left(X, \mathcal{A}_{X}\right)}\left(X, \mathcal{A}_{X}\right)=\mathcal{D}^{\text {perf }}\left(X, \mathcal{A}_{X}\right)=\mathcal{D}_{f E d /\left(X, \mathcal{A}_{X}\right)}\left(X, \mathcal{A}_{X}\right)$.

Corollary D.45. Let $f=f^{\prime} \circ f^{\prime \prime}$ be a decomposition of a morphism $f:\left(X, \mathcal{A}_{X}\right) \rightarrow\left(Y, \mathcal{A}_{Y}\right)$ with smooth $f^{\prime}:\left(X^{\prime}, \mathcal{A}_{X^{\prime}}\right) \rightarrow\left(Y, \mathcal{A}_{Y}\right)$ and finite $f^{\prime \prime}:\left(X, \mathcal{A}_{X}\right) \rightarrow\left(X^{\prime}, \mathcal{A}_{X^{\prime}}\right)$. Then $F \in \mathcal{D}_{f T d /\left(Y, \mathcal{A}_{Y}\right)}\left(X, \mathcal{A}_{X}\right) \Leftrightarrow$ $f_{*}^{\prime \prime} F \in \mathcal{D}^{\text {perf }}\left(X^{\prime}, \mathcal{A}_{X^{\prime}}\right) \Leftrightarrow F \in \mathcal{D}_{f E d /\left(Y, \mathcal{A}_{Y}\right)}\left(X, \mathcal{A}_{X}\right)$.

Proof: Use lemma D.43 and lemma D.40.

Corollary D.46. If $\phi:\left(W, \mathcal{A}_{W}\right) \rightarrow\left(Y, \mathcal{A}_{Y}\right)$ is a (strict and) faithful base change for a morphism $f:\left(X, \mathcal{A}_{X}\right) \rightarrow\left(Y, \mathcal{A}_{Y}\right)$ and $\left(Z, \mathcal{A}_{Z}\right)=\left(X, \mathcal{A}_{X}\right) \times_{\left(Y, \mathcal{A}_{Y}\right)}\left(W, \mathcal{A}_{W}\right)$ is the fiber product then we have $\phi^{*}\left(\mathcal{D}_{f T d /\left(Y, \mathcal{A}_{Y}\right)}\left(X, \mathcal{A}_{X}\right)\right) \subset \mathcal{D}_{f T d /\left(W, \mathcal{A}_{W}\right)}\left(Z, \mathcal{A}_{Z}\right)$, and $\phi^{*}\left(\mathcal{D}_{f E d /\left(Y, \mathcal{A}_{Y}\right)}\left(X, \mathcal{A}_{X}\right)\right) \subset \mathcal{D}_{f E d /\left(W, \mathcal{A}_{W}\right)}\left(Z, \mathcal{A}_{Z}\right)$.

Proof: Decompose $f:\left(X, \mathcal{A}_{X}\right) \rightarrow\left(Y, \mathcal{A}_{Y}\right)$ as a product of smooth $f^{\prime}:\left(X^{\prime}, \mathcal{A}_{X^{\prime}}\right) \rightarrow\left(Y, \mathcal{A}_{Y}\right)$ and finite $f^{\prime \prime}:\left(X, \mathcal{A}_{X}\right) \rightarrow\left(X^{\prime}, \mathcal{A}_{X^{\prime}}\right)$, and denote $\left(Z^{\prime}, \mathcal{A}_{Z^{\prime}}\right)=\left(X^{\prime}, \mathcal{A}_{X^{\prime}}\right) \times_{\left(Y, \mathcal{A}_{Y}\right)}\left(W, \mathcal{A}_{W}\right)$, the fiber product. Then we have a commutative diagram

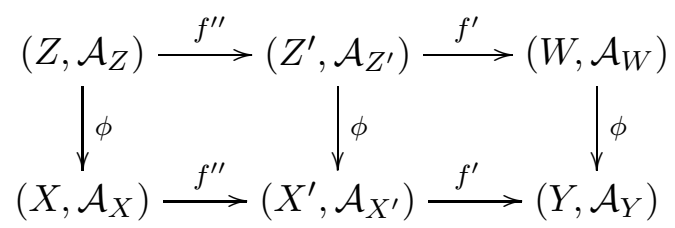

Its right square is exact cartesian because $f^{\prime}$ is smooth, and its left square is exact cartesian by lemma 2.25. Therefore $f_{*}^{\prime \prime} \phi^{*} F \cong \phi^{*} f_{*}^{\prime \prime} F$ and we deduce the claim from the criterion D.45.

Lemma D.47. If $\left(Y, \mathcal{A}_{Y}\right)$ is a smooth Azumaya variety then $\mathcal{D}_{f T d /\left(Y, \mathcal{A}_{Y}\right)}\left(X, \mathcal{A}_{X}\right)=\mathcal{D}^{b}\left(X, \mathcal{A}_{X}\right)=$ $\mathcal{D}_{f E d /\left(Y, \mathcal{A}_{Y}\right)}\left(X, \mathcal{A}_{X}\right)$.

Proof: If $F \in \mathcal{D}_{f T d /\left(Y, \mathcal{A}_{Y}\right)}\left(X, \mathcal{A}_{X}\right)$ then $F \cong F \otimes_{\mathcal{A}_{X}} \mathcal{A}_{X} \cong F \otimes_{\mathcal{A}_{X}} f^{*} \mathcal{A}_{Y}$ is bounded, therefore we have $\mathcal{D}_{f T d /\left(Y, \mathcal{A}_{Y}\right)}\left(X, \mathcal{A}_{X}\right) \subset \mathcal{D}^{b}\left(X, \mathcal{A}_{X}\right)$. On the other hand, take any $F \in \mathcal{D}^{[p, q]}\left(X, \mathcal{A}_{X}\right)$. Since $Y$ is smooth any $G \in \mathcal{D}^{[s, t]}\left(Y, \mathcal{A}_{Y}\right)$ is locally quasiisomorphic to a complex of locally projective $\mathcal{A}_{Y^{-}}$ modules concentrated in degrees $[s-\operatorname{dim} Y, t]$. Hence $F \otimes_{\mathcal{A}_{X}} f^{*} G \in \mathcal{D}^{[p+s-\operatorname{dim} Y, q+t]}\left(X, \mathcal{A}_{X}\right)$ and $F \in$ $\mathcal{D}_{f T d /\left(Y, \mathcal{A}_{Y}\right)}\left(X, \mathcal{A}_{X}\right)$.

\section{REFERENCES}

[B] A. Bondal, Representations of associative algebras and coherent sheaves, (Russian) Izv. Akad. Nauk SSSR Ser. Mat. 53 (1989), no. 1, 25-44; translation in Math. USSR-Izv. 34 (1990), no. 1, 23-42.

[BK] A. Bondal, M. Kapranov, Representable functors, Serre functors, and reconstructions, (Russian) Izv. Akad. Nauk SSSR Ser. Mat. 53 (1989), no. 6, 1183-1205, 1337; translation in Math. USSR-Izv. 35 (1990), no. 3, 519-541.

[BO1] A. Bondal, D. Orlov, Semiorthogonal decomposition for algebraic varieties, preprint math.AG/9506012.

[BO2] A. Bondal, D. Orlov, Derived categories of coherent sheaves, Proceedings of the International Congress of Mathematicians, Vol. II (Beijing, 2002), 47-56, Higher Ed. Press, Beijing, 2002.

[BO3] A. Bondal, D. Orlov, Reconstruction of a variety from the derived category and groups of autoequivalences, Compositio Math. 125 (2001), no. 3, 327-344. 
[BO4] A. Bondal, D. Orlov, private communication.

[Br1] T. Bridgeland, Equivalences of triangulated categories and Fourier-Mukai transforms, Bull. London Math. Soc. 31 (1999), no. 1, 25-34.

[BKR] T. Bridgeland, A. King, M. Ried, The McKay correspondence as an equivalence of derived categories, J. Amer. Math. Soc. 14 (2001), no. 3, 535-554.

[Ch] C. Chevalley, The algebraic theory of spinors, Columbia University Press, New York (1954).

[DR] U. Desale, S. Ramanan, Classification of vector bundles of rank 2 on hyperelliptic curves, Invent. Math. 38 $(1976 / 77)$, no. 2, 161-185.

[GSV] G. Gonzalez-Sprinberg, J-L. Verdier, Construction géométrique de la correspondence de McKay, Ann. Sc. ec. Norm. Sup. 16 (1983), 409-449.

[H] R. Hartshorn, Residues and duality, Lecture notes of a seminar on the work of A. Grothendieck, given at Harvard 1963/64. With an appendix by P. Deligne. Lecture Notes in Mathematics, No. 20 Springer-Verlag, Berlin-New York 1966.

[Ho] F. Holweck, Lieu singulier des variétés duales: approche géométrique et applications aux variétés homogènes, thesis.

$[\mathrm{Hu}] \quad$ J. Humphreys, Conjugacy classes in semisimple algebraic groups, Mathematical Surveys and Monographs, 43. American Mathematical Society, Providence, RI, 1995.

[KV] M. Kapranov, E. Vasserot, Kleinian singularities, derived categories and Hall algebras, Math. Ann. 316 (2000), no. 3, 565-576.

[Ki] A. King, Moduli of representations of finite-dimensional algebras, Quart. J. Math. Oxford Ser. (2) 45 (1994), no. 180, 515-530.

[K] A. Kuznetsov, Derived category of $V_{12}$ Fano threefolds, preprint math.AG/0310008.

[McK] J. McKay, Graphs, singularities and finite groups, Proc. Symp. Pure Math. 37 (1980), 183-186.

[OSS] C. Okonek, M. Schneider, H. Spindler, Vector bundles on complex projective spaces, Progress in Mathematics, 3. Birkhaüser, Boston, Mass., 1980.

[O1] D. Orlov, Exceptional set of vector bundles on the variety $V_{5}$, (Russian) Vestnik Moskov. Univ. Ser. I Mat. Mekh. 1991, no. 5, 69-71; translation in Moscow Univ. Math. Bull. 46 (1991), no. 5, 48-50.

[O2] D. Orlov, Projective bundles, monoidal transformations, and derived categories of coherent sheaves, (Russian) Izv. Ross. Akad. Nauk Ser. Mat. 56 (1992), no. 4, 852-862; translation in Russian Acad. Sci. Izv. Math. 41 (1993), no. 1, 133-141.

[O3] D. Orlov, Triangulated categories of singularities and D-branes in Landau-Ginzburg models, (Russian) Tr. Mat. Inst. Steklova 246 (2004), Algebr. Geom. Metody, Svyazi i Prilozh., 240-262; preprint math.AG/0302304.

[Ra] M. Razin, An exceptional collection on the Grassmannian of the Lie group $G_{2}$, preprint.

[S1] A. Samokhin, The derived category of coherent sheaves on $L G_{3}^{C}$, (Russian) Uspekhi Mat. Nauk 56 (2001), no. 3(339), 177-178; translation in Russian Math. Surveys 56 (2001), no. 3, 592-594.

[S2] A. Samokhin, Derived category of the hyperplane section of $L G_{3}^{C}$, preprint.

[T] A. Tyurin, The intersection of quadrics, (Russian) Uspehi Mat. Nauk 30 (1975), no. 6(186), 51-99.

Algebra Section, Steklov Mathematical Institute, 8 Gubkin str., Moscow 119991 Russia

E-mail address: akuznet@mi.ras.ru 